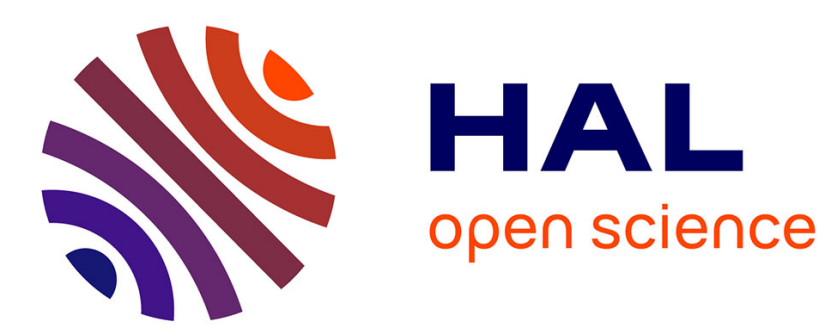

\title{
Quasi-linear perturbations of Hamiltonian Klein-Gordon equations on spheres
}

\author{
Jean-Marc Delort
}

\section{To cite this version:}

Jean-Marc Delort. Quasi-linear perturbations of Hamiltonian Klein-Gordon equations on spheres. 2011. hal-00643474

\author{
HAL Id: hal-00643474 \\ https://hal.science/hal-00643474
}

Preprint submitted on 22 Nov 2011

HAL is a multi-disciplinary open access archive for the deposit and dissemination of scientific research documents, whether they are published or not. The documents may come from teaching and research institutions in France or abroad, or from public or private research centers.
L'archive ouverte pluridisciplinaire HAL, est destinée au dépôt et à la diffusion de documents scientifiques de niveau recherche, publiés ou non, émanant des établissements d'enseignement et de recherche français ou étrangers, des laboratoires publics ou privés. 


\title{
Quasi-linear perturbations of Hamiltonian Klein-Gordon equations on spheres
}

\author{
J.-M. Delort \\ Université Paris 13, Institut Galilée, \\ CNRS, UMR 7539, Laboratoire Analyse Géométrie et Applications \\ 99, Avenue J.-B. Clément, \\ F-93430 Villetaneuse
}

\begin{abstract}
The Hamiltonian $\int_{X}\left(\left|\partial_{t} u\right|^{2}+|\nabla u|^{2}+\mathbf{m}^{2}|u|^{2}\right) d x$, defined on functions on $\mathbb{R} \times X$, where $X$ is a compact manifold, has critical points which are solutions of the linear Klein-Gordon equation. We consider perturbations of this Hamiltonian, given by polynomial expressions depending on first order derivatives of $u$. The associated PDE is then a quasi-linear KleinGordon equation. We show that, when $X$ is the sphere, and when the mass parameter $\mathbf{m}$ is outside an exceptional subset of zero measure, smooth Cauchy data of small size $\epsilon$ give rise to almost global solutions, i.e. solutions defined on a time interval of length $c_{N} \epsilon^{-N}$ for any $N$. Previous results were limited either to the semi-linear case (when the perturbation of the Hamiltonian depends only on $u$ ) or to the one dimensional problem.

The proof is based on a quasi-linear version of the Birkhoff normal forms method, relying on convenient generalizations of para-differential calculus.
\end{abstract}

\section{Introduction}

This paper is devoted to the study of solutions of small quasi-linear perturbations of an infinite dimensional Hamiltonian system. To be more specific, let $X$ be a compact Riemannian manifold, and define on $H^{s}(X, \mathbb{C})$, for $s$ large enough, $G_{0}(U)=\int_{X}\left(\Lambda_{\mathbf{m}} u\right) \bar{u} d \mu$, where $U=(u, \bar{u}), d \mu$ is the Riemannian volume and $\Lambda_{\mathbf{m}}=\sqrt{-\Delta_{X}+\mathbf{m}^{2}}$. Since $H^{s}(X, \mathbb{C})$ is endowed with a symplectic form $\omega_{0}\left(h, h^{\prime}\right)=2 \operatorname{Im} \int_{X} h \bar{h}^{\prime} d \mu$, one may consider the Hamiltonian equation associated to $G_{0}$, given by

$$
\partial_{t} u=i \nabla_{\bar{u}} G_{0}(u, \bar{u})
$$

If $u=\frac{\sqrt{2}}{2}\left(\Lambda_{\mathbf{m}}^{-1 / 2} \partial_{t} v+i \Lambda_{\mathbf{m}}^{1 / 2} v\right)$, with $v$ in $H^{s+1 / 2}(X, \mathbb{R})$ and $\partial_{t} v$ in $H^{s-1 / 2}(X, \mathbb{R})$, this Hamiltonian equation is nothing but the Klein-Gordon equation

$$
\left(\partial_{t}^{2}-\Delta+\mathbf{m}^{2}\right) v=0 .
$$

Keywords: Hamiltonian quasi-linear Klein-Gordon equations, Almost global existence, Birkhoff normal forms. MSC 35L72, 35S50, 37K45. 
We want to study the solutions of equations of form (1), where $G_{0}$ has been replaced by a more general Hamiltonian $G$, such that $G-G_{0}$ is small. By rescaling, this is equivalent to the study of

$$
\partial_{t} u=i \nabla_{\bar{u}} G(u, \bar{u}),
$$

where $G-G_{0}$ vanishes at least at order three at zero, and where the Cauchy data are small in $H^{s}(s \gg 1)$, of size $\epsilon \rightarrow 0$. The question is to determine whether, for $\epsilon$ small enough, the solution exists over a long interval of time, and has Sobolev norm $O(\epsilon)$ on that interval.

This problem has been quite extensively studied when the perturbation $G-G_{0}$ of the Hamiltonian is given by a function of $\Lambda_{\mathbf{m}}^{-1 / 2} U$ (while $G_{0}$ itself may be written as a function of $\Lambda_{\mathbf{m}}^{1 / 2} U$ ). At the level of the Hamiltonian equation, this corresponds to perturbations of (2) which are weakly semi-linear, i.e. may be written

$$
\left(\partial_{t}^{2}-\Delta+\mathbf{m}^{2}\right) v=f(v)
$$

for a smooth function $f$ vanishing at least at order 2 at zero. In one dimension, i.e. when $X$ is the circle, this problem has been solved by Bourgain [5] and Bambusi [1] (see also for related results Bambusi-Grébert [3] and the lectures of Grébert [16]). It has been proved that, if $\mathbf{m}$ is taken outside a subset of zero measure of $] 0,+\infty[$, for any $N \in \mathbb{N}$, for any $s$ large enough, there is $\epsilon_{0}>0$, such that equation (4) with Cauchy data of size $\epsilon<\epsilon_{0}$ in $H^{s+1 / 2}\left(\mathbb{S}^{1}, \mathbb{R}\right) \times H^{s-1 / 2}\left(\mathbb{S}^{1}, \mathbb{R}\right)$, has an almost global solution, i.e. a solution defined on a domain $]-T_{\epsilon}, T_{\epsilon}\left[\times \mathbb{S}^{1}\right.$, with $T_{\epsilon}>c \epsilon^{-N}$, satisfying an estimate

$$
\sup _{|t|<T_{\epsilon}}\|v(t, \cdot)\|_{H^{s+1 / 2}}+\sup _{|t|<T_{\epsilon}}\left\|\partial_{t} v(t, \cdot)\right\|_{H^{s-1 / 2}} \leq C \epsilon .
$$

This result has been extended to higher dimensions, i.e. to equation (4) on spheres, or more generally on Zoll manifolds, by Bambusi, Delort, Grébert and Szeftel [2], after preliminary results of Delort and Szeftel $[8,9,10]$. The method of proof relies on Birkhoff normal forms, as in one dimension, to reduce the proof of long time existence and of estimate (5) to the study of a sequence of homological equations. The resolution of these equations is possible only under a suitable small divisors property, which holds when the parameter $\mathbf{m}$ is outside a subset of zero measure, because of the very special distribution of the eigenvalues of $-\Delta$ on $\mathbb{S}^{d}$. Actually, the important property is that two different eigenvalues of $\sqrt{-\Delta_{\mathbb{S}^{d}}}$ are separated essentially by a fixed distance. When such a property does not hold, for instance when $X=\mathbb{T}^{d}$, or when $-\Delta$ is replaced by the harmonic oscillator $-\Delta+|x|^{2}$ on $X=\mathbb{R}^{d}$, only much weaker results are known (see $[6,12,20])$.

Let us mention also that normal forms methods have been proved useful to study long time existence for solutions of equations of the form (4) on $\mathbb{R}^{d}$, for Cauchy data that are smooth and decaying at infinity. Actually, the use of normal forms in that framework has been introduced by Shatah [19] to prove global existence of solutions of non-linear Klein-Gordon equations on $\mathbb{R}^{3}$ (an alternative proof has been given at the same time by Klainerman [18]). More recently, this approach has been made more systematic in a series of papers of Germain, Masmoudi and Shatah $[14,15]$ and Germain [13].

Let us go back to our Hamiltonian equation (4). We would like to study a more general version of (4), with a right hand side depending also on derivatives of $v$. This corresponds to an equation 
of form (3) where the perturbation $G-G_{0}$ of $G_{0}$ has the same strength, relatively to the number of derivatives involved, as $G_{0}$ itself, i.e. is a function of $\Lambda_{\mathbf{m}}^{1 / 2} U$ instead of $\Lambda_{\mathbf{m}}^{-1 / 2} U$. The associated Hamiltonian equation is like (4), where the right hand side is replaced by a quasi-linear nonlinearity, i.e. an expression depending on second order derivatives of $v$, which is linear with respect to $\left(\partial^{\alpha} v\right)_{|\alpha|=2}$. This problem has been solved in one dimension in [7]. The goal of this paper is to obtain a similar almost global existence result on any higher dimensional sphere $\mathbb{S}^{d}$.

Let us describe the main new ideas that have to be introduced in comparison with our previous paper [7]. As in this work, the key point is to design a Birkhoff normal forms method adapted to quasi-linear equations. This requires to express the Hamiltonian using para-differential operators. In one dimension, one may use Fourier analysis on $\mathbb{S}^{1}$ to define such classes of operators globally on $\mathbb{S}^{1}$, using symbols which are functions on $\mathbb{S}^{1} \times \mathbb{Z}$ (where $\mathbb{Z}$ should be considered as the dual group of $\mathbb{S}^{1}$ ). In higher dimensions, one can no longer do so. Instead, we define para-differential operators using a characterization in terms of commutators with differential operators, similar to the Beals characterization of pseudo-differential operators [4]. The classes we need are more general than usual para-differential operators: they depend on some auxiliary functions, and have to take into account some losses relatively to small frequencies that will appear because of small divisors. Because of that, we have to rewrite the whole theory (symbolic calculus, principal symbols on compact manifolds,...) in our framework. This is done in section 2 of the paper. Section 3 is devoted to the computation of Poisson brackets between functions defined in terms of integrals of type $\int_{X}(A(U) u) \bar{u} d \mu, \operatorname{Re} \int_{X}(A(U) u) u d \mu$, where $A(U)$ is a para-differential operator of order one, homogeneous of some degree $k$ in $U$. The proof of the main theorem of long time existence occupies section 4. Let us describe the main new idea on a toy model.

Consider equation (3) with Hamiltonian

$$
G(u, \bar{u})=\int_{X}\left(\Lambda_{\mathbf{m}} u\right) \bar{u} d \mu+\operatorname{Re} \int_{X}(A(U) u) \bar{u} d \mu,
$$

where $A(U)$ is a self-adjoint para-differential operator of order one, homogeneous of degree $k$ in $U=(u, \bar{u})$. Equation $(3)$ reads

$$
D_{t} u=\Lambda_{\mathbf{m}} u+A(U) u+\cdots
$$

where the dots represent some contributions which are of a semi-linear nature (opposed to the main quasi-linear contribution $A(U) u$ ). To prove that (7) with $H^{s}$ data of size $\epsilon$ has a solution defined on a time interval of length $c \epsilon^{-N}$, one has to find a modified energy $\Theta_{s}(u)$ such that $\Theta_{s}(u) \sim\|u\|_{H^{s}}^{2}$ close to zero and

$$
\frac{d}{d t} \Theta_{s}(u(t, \cdot))=O\left(\|u(t, \cdot)\|_{H^{s}}^{N+2}\right)
$$

when $u$ is a solution of (7). The usual normal forms method consists in defining $\Theta_{s}=\Theta_{s}^{0} \circ \chi$, where $\Theta_{s}^{0}=\left\|\Lambda_{\mathbf{m}}^{s} u\right\|_{L^{2}}^{2}$ is the usual $H^{s}$-energy, and $\chi$ is a convenient symplectic diffeomorphism on a neighborhood of zero in $H^{s}$, defined as the value at time $t=-1$ of the flow of the Hamiltonian vector field $X_{F}$ of some auxiliary function $F$. Then equation (3) implies

$$
\frac{d}{d t} \Theta_{s}(u(t, \cdot))=\left\{\Theta_{s}^{0} \circ \chi, G\right\}(u(t, \cdot))=\left\{\Theta_{s}^{0}, G \circ \chi^{-1}\right\} \circ \chi(u(t, \cdot))
$$


and the definition of $\chi$ gives an expansion

$$
\left\{\Theta_{s}^{0}, G \circ \chi^{-1}\right\} \sim\left\{\Theta_{s}^{0}, \sum_{p} \frac{(-1)^{p}}{p !} \operatorname{ad}^{p} F \cdot G\right\}
$$

where $\operatorname{ad} F \cdot G=\{F, G\}$. One looks for $F$ as given by an expansion in terms of homogeneous terms $F=\sum_{\ell \geq 1} F_{\ell}$. Then the term of degree of homogeneity $p+2$ in (10) vanishes if and only if

$$
\left\{\Theta_{s}^{0},-\left\{F_{p}, G_{0}\right\}+H_{p}\right\}=0,
$$

where $H_{p}$ is computed from $G$ and $F_{p^{\prime}}$ with $p^{\prime}<p$. One looks for $F_{p}$ as

$$
F_{p}(u, \bar{u})=\int_{X}\left(B_{p}(U) u\right) \bar{u} d \mu
$$

where $B_{p}(U)$ is a self-adjoint para-differential operator of order one. One proves moreover that, if $F_{p^{\prime}}$ has a similar structure for $p^{\prime}<p$, then

$$
H_{p}(u, \bar{u})=\int_{X}\left(A_{p}^{\prime}(U) u\right) \bar{u} d \mu+\cdots
$$

where $A_{p}^{\prime}$ is also a self-adjoint operator of first order, and the dots represent some other contributions which are of a semi-linear nature. Then $\left\{F_{p}, G_{0}\right\}-H_{p}$ may be written as $\int_{X}\left(C_{p}(U) u\right) \bar{u} d \mu$ where, if we decompose $B_{p}(U)=\sum_{\ell=0}^{p} B_{p}^{\ell}(\underbrace{u, \ldots, u}_{\ell}, \bar{u}, \ldots, \bar{u})$, and make similar decompositions of $A_{p}^{\prime}, C_{p}$,

$$
\begin{array}{r}
C_{p}^{\ell}(u, \ldots, \bar{u})=i\left[B_{p}^{\ell}, \Lambda_{\mathbf{m}}\right](u, \ldots, \bar{u})+i \sum_{j=1}^{\ell} B_{p}^{\ell}(u, \ldots, \underbrace{\Lambda_{\mathbf{m}} u}_{j}, \ldots, \bar{u}) \\
-i \sum_{j=\ell+1}^{p} B_{p}^{\ell}(u, \ldots, \underbrace{\Lambda_{\mathbf{m}} \bar{u}}_{j}, \ldots, \bar{u})-A_{p}^{\ell \ell}(u, \ldots, \bar{u}) .
\end{array}
$$

A way to solve (11) would be to find $B_{p}^{\ell}$ so that $C_{p}^{\ell}=0$. If one replaces in $(13)(u, \ldots, \bar{u})$ by $\left(\Pi_{n_{1}} u, \ldots, \Pi_{n_{p}} \bar{u}\right)$, where $\Pi_{n}$ is the spectral projector associated by the $n$-th eigenvalue $\lambda_{n}^{2}$ of $-\Delta$, one gets to show that

$$
\left[B_{p}^{\ell}, \Lambda_{\mathbf{m}}\right]\left(\Pi_{n_{1}} u, \ldots, \Pi_{n_{p}} \bar{u}\right)+G_{\mathbf{m}}^{p, \ell}\left(n^{\prime}\right) B_{p}^{\ell}\left(\Pi_{n_{1}} u, \ldots, \Pi_{n_{p}} \bar{u}\right)=-i A_{p}^{\prime \ell}\left(\Pi_{n_{1}} u, \ldots, \Pi_{n_{p}} \bar{u}\right),
$$

where $G_{\mathbf{m}}^{p, \ell}\left(n^{\prime}\right)=\sum_{j=1}^{\ell} \sqrt{\mathbf{m}^{2}+\lambda_{n_{j}}^{2}}-\sum_{j=\ell+1}^{p} \sqrt{\mathbf{m}^{2}+\lambda_{n_{j}}^{2}}$. One may choose $\mathbf{m}$ so that, for some $c>0, L_{0}>0$,

$$
d\left(\mathbb{Z}, G_{\mathbf{m}}^{p, \ell}\left(n^{\prime}\right)\right) \geq c\left|n^{\prime}\right|^{-L_{0}}=c\left(n_{1}+\cdots+n_{p}\right)^{-L_{0}}
$$

as soon as the left hand side does not vanish trivially (i.e. as soon as trivial two by two cancellations in the expression of $G_{\mathbf{m}}^{p, \ell}\left(n^{\prime}\right)$ are excluded). If one replaces in the bracket in (14), $\Lambda_{\mathbf{m}}$ by $\tilde{\Lambda}=\sqrt{-\Delta+\left(\frac{d-1}{2}\right)^{2}}$, an approximate solution of (14) may be obtained defining

$$
B_{p}^{\ell}\left(\Pi_{n_{1}} u, \ldots, \Pi_{n_{p}} \bar{u}\right)=-i \int_{0}^{+\infty} e^{-i t \tilde{\Lambda}} A_{p}^{\prime \ell}\left(\Pi_{n_{1}} u, \ldots, \Pi_{n_{p}} \bar{u}\right) e^{i t \tilde{\Lambda}} e^{i t G_{\mathbf{m}}^{p \ell \ell}\left(n^{\prime}\right)} \theta(\epsilon t) d t
$$


where $\theta \in C_{0}^{\infty}(\mathbb{R}), \theta \equiv 1$ close to zero. Actually, using that the eigenvalues of $\widetilde{\Lambda}$ are half integers, we see that $t \rightarrow e^{-i t \widetilde{\Lambda}} A_{p}^{\prime} \ell e^{i t \widetilde{\Lambda}}$ is $4 \pi$-periodic. Decomposing this function of time in Fourier series and using the small divisor estimate (15), we check that $B_{k}^{\ell}$ is a para-differential operator, and that (14) holds, up to some remainders. Of course, this cannot be done when estimate (15) does not hold i.e. when there is a trivial vanishing of the left hand side. But the contributions corresponding to this special case are in the kernel of $\left\{\Theta_{s}^{0}, \cdot\right\}$, so may be discarded since the equation we need to solve is $(11)$ and not $\left\{F_{p}, G_{0}\right\}-H_{p}=0$.

Using repeatedly the preceding method, we may eliminate as many terms as we want in the right hand side of (10), and deduce from (9) the wanted property (8).

Let us point out that several other difficulties have to be dealt with. First, if one really wanted to define $\chi$ as the value at time -1 of the flow of $X_{F}$, with $F=\sum_{p>1} F_{p}$ and $F_{p}$ given by (12), one would have to solve an equation $\dot{u}=X_{F}(u)$ which is not an ODE, at the difference with the case of semi-linear equations. This comes from the fact that in (12), $B_{p}$ is of order one, so that $X_{F}$ involves the loss of one derivative. We circumvent this problem defining not $\chi$ itself, but only its action by right composition on functions, using iterated Poisson brackets as in (10).

Another complication that appears when solving the "real problem", instead of the toy model (6), is that the Hamiltonian one has to study is of the form

$$
G(u, \bar{u})=\int_{X}\left(\Lambda_{\mathbf{m}} u\right) \bar{u} d \mu+\operatorname{Re} \int_{X}(A(U) u) \bar{u} d \mu+\operatorname{Re} \int_{X}(C(U) u) u d \mu,
$$

where $C(U)$ is also a para-differential operator of order one, vanishing at $U=0$. Before performing the method we outlined above, one has first to reduce oneself to an Hamiltonian of form (6). One does that in two steps. First, one eliminates the Taylor expansion of $U \rightarrow C(U)$ at $U=0$ up to a large enough degree. This is done by a Birkhoff normal form method, involving functions of type (12), but with $B_{p}$ of order zero instead of one. Because of that, this step is purely semi-linear. When this has been achieved, one obtains still a reduced Hamiltonian of form (16), but with a $C$ vanishing at large order at $U=0$. One then makes a change of unknown $U \rightarrow \tilde{U}=\Psi(U)$, constructed in such a way that the new Hamiltonian is a function of $\tilde{U}$ of form (6). This part of the reasoning is similar to the usual process of diagonalization of the principal symbol of a quasi-linear symmetrizable system, that allows one to prove energy inequalities in such a framework. Actually, the construction of the change of unknown $\Psi$ is made from a convenient diagonalization of that type.

Finally, let us mention that we limited ourselves to polynomial non-linearities and to spheres (instead of Zoll manifolds) to avoid some extra technicalities.

\section{Statement of the main theorem}

\subsection{Notations. First statement}

We denote by $(X, g)$ the standard $d$-dimensional sphere $(d \geq 2)$, endowed with its usual metric, by $\Delta$ the Laplace-Beltrami operator on $X$ and by $d \mu$ the volume form associated to the metric, 
given in local coordinates by $d \mu=(\operatorname{det} g)^{1 / 2} d x$. The eigenvalues of $-\Delta$ are given by $\lambda_{n}^{2}=$ $(n-1)(n+d-2), n \in \mathbb{N}^{*}$. Let $f: \mathbb{R} \times T^{*} X \rightarrow \mathbb{R}$ be a smooth function, $(z, \rho) \rightarrow f(z, \rho)$, polynomial relatively to $z$ and to the fiber variable of $T^{*} X$. Assume that $f$ vanishes at least at order 3 on $\{0\} \times X$ (where $X$ is considered as the zero section of $T^{*} X$ ). For $v: X \rightarrow \mathbb{R}$ a smooth enough function, we define from $f, v$ a new function $P[v]: X \rightarrow \mathbb{R}$ in the following way: If $x$ denotes local coordinates on an open set $U$ of $X$, if $\rho=(x, \xi)$ are the corresponding local coordinates on $T^{*} U, f$ is a function of $(z, x, \xi)$ on $\mathbb{R} \times T^{*} U$, and we set

$$
P[v](x)=\frac{\partial f}{\partial z}\left(v(x), x, \frac{\partial v}{\partial x}(x)\right)-\sum_{j=1}^{d}(\operatorname{det} g)^{-1 / 2} \frac{\partial}{\partial x_{j}}\left[(\operatorname{det} g)^{1 / 2} \frac{\partial f}{\partial \xi_{j}}\left(v(x), x, \frac{\partial v}{\partial x}(x)\right)\right] .
$$

We notice that $P[v]$ is intrinsically defined. Actually, it suffices to check that, for any smooth function $h$ on $X$, compactly supported in the local chart $U, \int_{X} P[v](x) h(x) d \mu(x)$ is intrinsically defined. Denote by $\{\cdot, \cdot\}$ the Poisson bracket on $T^{*} X$, given in local coordinates by

$$
\{f, g\}=\sum_{j=1}^{d}\left(\frac{\partial f}{\partial \xi_{j}} \frac{\partial g}{\partial x_{j}}-\frac{\partial f}{\partial x_{j}} \frac{\partial g}{\partial \xi_{j}}\right) .
$$

Then it follows from (1.1.1) and the definition of $d \mu$ that

$$
\int_{X} P[v] h d \mu=\int_{X} \frac{\partial f}{\partial z}(v, d v) h d \mu+\int_{X}\{f, h\}(v, d v) d \mu
$$

which is intrinsic (We denote by $d v$ the section of $T^{*} X$ given in local coordinates by $x \rightarrow$ $\left.\left(x, \frac{\partial v}{\partial x}(x)\right)\right)$.

Our main result is the following one. For $s$ in $\mathbb{R}$, we denote by $H^{s}(X, \mathbb{R})$ the Sobolev space of real valued functions on $X$.

Theorem 1.1.1 There is a subset $\mathcal{N}$ of zero measure in $] 0,+\infty[$ and for any $\mathbf{m} \in] 0,+\infty[-\mathcal{N}$, any $P \in \mathbb{N}$, there is $s_{0}>0$ such that, for any $s \geq s_{0}$, there are $\epsilon_{0}>0, c>0, C>0$ and, for any $\epsilon \in] 0, \epsilon_{0}\left[\right.$, any $\left(v_{0}, v_{1}\right)$ in the unit ball of $H^{s+\frac{1}{2}}(X, \mathbb{R}) \times H^{s-\frac{1}{2}}(X, \mathbb{R})$, there is a unique solution $v$ in $C^{0}(]-T_{\epsilon}, T_{\epsilon}\left[, H^{s+\frac{1}{2}}\right) \cap C^{1}(]-T_{\epsilon}, T_{\epsilon}\left[, H^{s-\frac{1}{2}}\right)$ of the equation

$$
\begin{aligned}
\left(\partial_{t}^{2}-\Delta+\mathbf{m}^{2}\right) v & =P[v] \\
\left.v\right|_{t=0} & =\epsilon v_{0} \\
\left.\partial_{t} v\right|_{t=0} & =\epsilon v_{1}
\end{aligned}
$$

with $T_{\epsilon} \geq c \epsilon^{-P}$. Moreover, $v$ satisfies the uniform bound

$$
\sup _{]-T_{\epsilon}, T_{\epsilon}[}\|v(t, \cdot)\|_{H^{s+1 / 2}}+\sup _{]-T_{\epsilon}, T_{\epsilon}[}\left\|\partial_{t} v(t, \cdot)\right\|_{H^{s-1 / 2}} \leq C \epsilon .
$$

Remark: The assumption on $f$, together with (1.1.1), shows that the right hand side of the first equation in (1.1.3) is a quasi-linear (polynomial) non-linearity, vanishing at least at order two at zero. 
Expression (1.1.1) of the non-linearity will allow us to write equation (1.1.3) as a Hamiltonian system. Let us introduce the necessary notations.

Denote by $J$ the matrix

$$
J=\left[\begin{array}{cc}
0 & -1 \\
1 & 0
\end{array}\right]
$$

and for $Z, Z^{\prime}$ two functions in $L^{2}\left(X, \mathbb{R}^{2}\right)$, define

$$
\omega_{0}\left(Z, Z^{\prime}\right)=\left\langle{ }^{t} J Z, Z^{\prime}\right\rangle=\left\langle Z, J Z^{\prime}\right\rangle,
$$

where $\langle\cdot, \cdot\rangle$ is the $L^{2}\left(X, \mathbb{R}^{2}\right)$ scalar product given by integration against the measure $d \mu$. Let $s \in \mathbb{R}_{+}, \Omega$ be an open subset of $H^{s}\left(X, \mathbb{R}^{2}\right), F: \Omega \rightarrow \mathbb{R}$ a $C^{1}$ function. We define the symplectic gradient $X_{F}(V)$ of $F$ at $V \in \Omega$ as the element of $\mathcal{D}^{\prime}\left(X, \mathbb{R}^{2}\right)$ defined by $\omega_{0}\left(X_{F}(V), Z\right)=d F(V) \cdot Z$ for any $Z$ in $C^{\infty}\left(X, \mathbb{R}^{2}\right)$. In an equivalent way

$$
X_{F}(V)=J \nabla F(V) .
$$

If $G: \Omega \rightarrow \mathbb{R}$ is another $C^{1}$ function, we define the Poisson bracket of $F$ and $G$ by

$$
\{F, G\}(V)=d F \cdot X_{G}(V)=d F(V) \cdot[J \nabla G(V)]
$$

as soon as the right hand side has a meaning, i.e. when $X_{G}(V)$ belongs to a subspace of $\mathcal{D}^{\prime}(X, \mathbb{R})$ to which the linear form $d F(V)$ extends. Of course, this Poisson bracket should not be confused, though we use the same notation, with the Poisson bracket on $T^{*} X$ used in (1.1.2).

Let us rewrite equation (1.1.3) in the Hamiltonian framework. Let

$$
\Lambda_{\mathbf{m}}=\sqrt{-\Delta+\mathbf{m}^{2}}
$$

and define from the function $v$ solving (1.1.3), an element $V$ of $H^{s}\left(X, \mathbb{R}^{2}\right)$ by

$$
V(t, x)=\left[\begin{array}{c}
\Lambda_{\mathbf{m}}^{-1 / 2} \partial_{t} v \\
\Lambda_{\mathbf{m}}^{1 / 2} v
\end{array}\right]=\left[\begin{array}{c}
V^{1} \\
V^{2}
\end{array}\right] .
$$

If we set $F(v)=\int_{X} f(v, d v) d \mu$, and if $h \in C^{\infty}(X, \mathbb{R})$,

$$
d F(v) \cdot h=\int_{X} P[v] h d \mu
$$

as follows from (1.1.1), taking $h$ supported in a local chart domain. We define for $V$ in $H^{s}\left(X, \mathbb{R}^{2}\right)$, with $s$ large enough

$$
\begin{aligned}
G_{0}(V) & =\frac{1}{2} \int_{X}\left(\Lambda_{\mathbf{m}} V\right) \cdot V d \mu \\
G(V) & =G_{0}(V)-F\left(\Lambda_{\mathbf{m}}^{-1 / 2} V^{2}\right) .
\end{aligned}
$$

Then, if $H$ is in $C^{\infty}\left(M, \mathbb{R}^{2}\right)$,

$$
d G(V) \cdot H=\int_{X}\left(\Lambda_{\mathbf{m}} V\right) \cdot H d \mu-\int_{X} P\left[\Lambda_{\mathbf{m}}^{-1 / 2} V^{2}\right]\left(\Lambda_{\mathbf{m}}^{-1 / 2} H^{2}\right) d \mu
$$


which shows that

$$
\nabla G(V)=\Lambda_{\mathbf{m}} V+\left[\begin{array}{c}
0 \\
-\Lambda_{\mathbf{m}}^{-1 / 2} P\left[\Lambda_{\mathbf{m}}^{-1 / 2} V^{2}\right]
\end{array}\right] .
$$

Using (1.1.10), we see that $v$ is a solution of the first equation in (1.1.3) if and only if $V$ satisfies

$$
\partial_{t} V=X_{G}(V) .
$$

Let us write this equality using complex coordinates. We identify $H^{s}\left(X, \mathbb{R}^{2}\right)$ to $H^{s}(X) \stackrel{\text { def }}{=}$ $H^{s}(X, \mathbb{C})$ through

$$
V=\left[\begin{array}{l}
V^{1} \\
V^{2}
\end{array}\right] \rightarrow u=\frac{\sqrt{2}}{2}\left(V^{1}+i V^{2}\right) .
$$

More precisely, we identify $H^{s}\left(X, \mathbb{R}^{2}\right)$ to the submanifold $\left\{\left(U^{1}, U^{2}\right) ; U^{2}=\overline{U^{1}}\right\}$ inside the product $H^{s}(X, \mathbb{C}) \times H^{s}(X, \mathbb{C})$ through

$$
V=\left[\begin{array}{l}
V^{1} \\
V^{2}
\end{array}\right] \rightarrow U=\left[\begin{array}{l}
u=\frac{\sqrt{2}}{2}\left(V^{1}+i V^{2}\right) \\
\bar{u}=\frac{\sqrt{2}}{2}\left(V^{1}-i V^{2}\right)
\end{array}\right]
$$

If $F$ is a $C^{1}$ function on an open subset of $H^{s}\left(X, \mathbb{R}^{2}\right)$, we define

$$
\begin{gathered}
d_{u} F=\frac{\sqrt{2}}{2}\left[d_{V^{1}} F-i d_{V^{2}} F\right], d_{\bar{u}} F=\frac{\sqrt{2}}{2}\left[d_{V^{1}} F+i d_{V^{2}} F\right] \\
\nabla_{u} F=\frac{\sqrt{2}}{2}\left[\nabla_{V^{1}} F-i \nabla_{V^{2}} F\right], \nabla_{\bar{u}} F=\frac{\sqrt{2}}{2}\left[\nabla_{V^{1}} F+i \nabla_{V^{2}} F\right] .
\end{gathered}
$$

If $\left[\begin{array}{l}H_{1} \\ H_{2}\end{array}\right]$ (resp. $\left.\left[\begin{array}{l}H_{1}^{\prime} \\ H_{2}^{\prime}\end{array}\right]\right)$, element of $H^{s}\left(X, \mathbb{R}^{2}\right)$, is identified through (1.1.14) to the element $H=\left[\begin{array}{l}h \\ \bar{h}\end{array}\right]$ (resp. $H^{\prime}=\left[\begin{array}{l}h^{\prime} \\ h^{\prime}\end{array}\right]$ ) of $H^{s}(X)$, the symplectic form in complex coordinates may be written

$$
\omega_{0}\left(H, H^{\prime}\right)=i\left\langle H, J H^{\prime}\right\rangle .
$$

Through identification (1.1.14), the expression of the Hamiltonian vector field in complex coordinates is given by

$$
X_{F}(U)=-i J\left[\begin{array}{c}
\nabla_{u} F \\
\nabla \bar{u} F
\end{array}\right]=i^{t} J\left[\begin{array}{c}
\nabla_{u} F \\
\nabla_{\bar{u}} F
\end{array}\right]=i\left[\begin{array}{c}
\nabla_{\bar{u}} F \\
-\nabla_{u} F
\end{array}\right] .
$$

If $F$ and $G$ are two $C^{1}$ functions on an open set of $H^{s}(X)$, whose differentials at every point extend as bounded maps on $L^{2}(X)$, one gets the following expression for their Poisson brackets in complex coordinates

$$
\{F, G\}=d F \cdot X_{G}(U)=i\left[d_{u} F \cdot \nabla_{\bar{u}} G-d_{\bar{u}} F \cdot \nabla_{u} G\right] .
$$

Finally, equation (1.1.12) may be written in an equivalent way

$$
\partial_{t} u=i \nabla_{\bar{u}} G(u, \bar{u})
$$

if we consider the function given by (1.1.11) as a function of the complex variable $U=(u, \bar{u})$. 


\subsection{Quasi-linear Hamiltonians and general statements}

The goal of this subsection is to introduce a general class of Hamiltonian equations, containing (1.1.11), such that the associated equation (1.1.12) or (1.1.18) is a quasi-linear first order system. This class has to be wide enough to be stable under the reductions that will have to be made in the proof of Theorem 1.1.1.

Let us introduce some notation. We denoted by $\lambda_{n}=\sqrt{(n-1)(n+d-2)}, n \in \mathbb{N}^{*}$, the eigenvalues of $\sqrt{-\Delta}$ on $X=\mathbb{S}^{d}$. We call $\Pi_{n}$ the spectral projector associated to $\lambda_{n}$. In particular, for any $s \in \mathbb{R}$, there is $C>0$ such that, for any $u$ in $H^{s}(X)$,

$$
C^{-1} n^{s}\left\|\Pi_{n} u\right\|_{L^{2}} \leq\left\|\Pi_{n} u\right\|_{H^{s}} \leq C n^{s}\left\|\Pi_{n} u\right\|_{L^{2}} .
$$

Fix $\varphi$ a non-negative real valued smooth function on $\mathbb{R}$, compactly supported in $] 0,+\infty[$, such that $\sum_{j=-\infty}^{+\infty} \varphi\left(2^{-j} t\right) \equiv 1$ for any $t>0$. We define $\psi(t)=\sum_{j=-\infty}^{0} \varphi\left(2^{-j} t\right)$ and set for $u \in \mathcal{D}^{\prime}(X)$, $j \in \mathbb{N}$,

$$
\begin{aligned}
& \Delta_{j} u=\sum_{n=1}^{+\infty} \varphi\left(2^{-j} \lambda_{n}\right) \Pi_{n} u, j \geq 1 \\
& \Delta_{0} u=S_{0} u=\sum_{n=1}^{+\infty} \psi\left(\lambda_{n}\right) \Pi_{n} u, \\
& S_{j} u=\sum_{j^{\prime}=0}^{j} \Delta_{j^{\prime}} u, j \geq 0 .
\end{aligned}
$$

In that way, $u$ is in $H^{s}(X)$ if and only if $\left(2^{-j s}\left\|\Delta_{j} u\right\|_{L^{2}}\right)_{j}$ is in $\ell^{2}(\mathbb{N})$.

We shall study an equation of the form of (1.1.18) where the Hamiltonian $G$ will be expressed in terms of para-differential operators acting on $U=\left[\begin{array}{l}u \\ u\end{array}\right]$. We introduce several classes of multilinear operators. If $p \in \mathbb{N}^{*}$, if $\mathcal{U}=\left(u_{1}, \ldots, u_{p}\right)$ is a $p$-tuple of complex valued functions defined on $X$ and if $n^{\prime}=\left(n_{1}, \ldots, n_{p}\right)$ is an element of $\left(\mathbb{N}^{*}\right)^{p}$, we denote

$$
\Pi_{n^{\prime}} \mathcal{U}=\left(\Pi_{n_{1}} u_{1}, \ldots, \Pi_{n_{p}} u_{p}\right),\left|n^{\prime}\right|=\max \left(n_{1}, \ldots, n_{p}\right) .
$$

We assume given a strictly increasing function $\nu: \mathbb{N} \rightarrow \mathbb{N}$, such that $\nu(0)=0$, that satisfies a growth condition of type

$$
\nu(p)+\nu(q)+a \leq \nu(p+q), p, q \in \mathbb{N}^{*},
$$

where $a$ is some positive constant that will be adjusted later.

Definition 1.2.1 Let $p \in \mathbb{N}, m \in \mathbb{R}, \nu \in \mathbb{R}_{+}$. One denotes by $\widetilde{\mathcal{P}}_{p}^{m, \nu}$ the space of $p$-linear maps $\mathcal{U}=\left(u_{1}, \ldots, u_{p}\right) \rightarrow A(\mathcal{U})$, defined on $C^{\infty}(X)^{p}$ with values in the space of linear maps from $C^{\infty}(X)$ to $\mathcal{D}^{\prime}(X)$, such that, for any $N_{1}, N_{2}$ in $\mathbb{N}$, there is $C>0$ so that, for any $\mathcal{U}=$ $\left(u_{1}, \ldots, u_{p}\right)$, any $\left(n_{0}, \ldots, n_{p+1}\right)$ in $\left(\mathbb{N}^{*}\right)^{p+2}$,

$$
\begin{aligned}
\left\|\Pi_{n_{0}} A\left(\Pi_{n^{\prime}} \mathcal{U}\right) \Pi_{n_{p+1}}\right\|_{\mathcal{L}\left(L^{2}\right)} \leq & C\left\langle n_{0}-n_{p+1}\right\rangle^{-2}\left(\min \left[\frac{n_{0}}{n_{p+1}}, \frac{n_{p+1}}{n_{0}}\right]\right)^{N_{1}} n_{0}^{m} \\
& \times\left|n^{\prime}\right|^{\nu(p)+\nu}\left(1+\frac{\left|n^{\prime}\right|}{n_{0}}\right)^{-N_{2}} \prod_{\ell=1}^{p}\left\|\Pi_{n_{\ell}} u_{\ell}\right\|_{L^{2}}
\end{aligned}
$$


where $\langle n\rangle=\left(1+n^{2}\right)^{1 / 2}$.

Remarks: • The definition implies that for any $j, j^{\prime}$ in $\mathbb{N}$

$$
\left\|\Delta_{j} A\left(\Pi_{n^{\prime}} \mathcal{U}\right) \Delta_{j^{\prime}}\right\|_{\mathcal{L}\left(L^{2}\right)} \leq C 2^{-N_{1}\left|j-j^{\prime}\right|} 2^{j m}\left|n^{\prime}\right|^{\nu(p)+\nu}\left(1+2^{-j}\left|n^{\prime}\right|\right)^{-N_{2}} \prod_{\ell=1}^{p}\left\|\Pi_{n_{\ell}} u_{\ell}\right\|_{L^{2}}
$$

The characterization of Sobolev spaces in terms of dyadic decompositions implies that, for any $s$ in $\mathbb{R}$, any $N_{2} \in \mathbb{N},\left|n^{\prime}\right|^{N_{2}} A\left(\Pi_{n^{\prime}} \mathcal{U}\right)$ extends as a bounded linear map from $H^{s}(X)$ to $H^{s-m-N_{2}}(X)$, with estimates

$$
\left|n^{\prime}\right|^{N_{2}}\left\|A\left(\Pi_{n^{\prime}} \mathcal{U}\right)\right\|_{\mathcal{L}\left(H^{s}, H^{\left.s-m-N_{2}\right)}\right.} \leq C_{s, N_{2}}\left|n^{\prime}\right|^{\nu(p)+\nu} \prod_{\ell=1}^{p}\left\|\Pi_{n_{\ell}} u_{\ell}\right\|_{L^{2}} .
$$

In particular, if $u_{\ell} \in H^{\sigma}(X), \ell=1, \ldots, p$, with $\sigma>\nu(p)+\nu+\frac{1}{2}, A(\mathcal{U})=\sum_{n^{\prime}} A\left(\Pi_{n^{\prime}} \mathcal{U}\right)$ defines an element of $\mathcal{L}\left(H^{s}, H^{s-m}\right)$ for any $s$ in $\mathbb{R}$.

- Estimates $(1.2 .6),(1.2 .7)$ assert that elements of $\widetilde{\mathcal{P}}_{p}^{0, \nu}$ are bounded from $H^{s}$ to $H^{s}$ for any $s$, and that in (1.2.6), the coefficients $\mathcal{U}$ are spectrally localized essentially for frequencies $\lambda_{n_{1}}, \ldots, \lambda_{n_{p}}$ satisfying $\left|n^{\prime}\right|=\max \left(n_{1}, \ldots, n_{p}\right) \leq 2^{j}$. Such properties are to be expected from para-differential operators. Nevertheless, they do not suffice to give a class of operators enjoying a symbolic calculus. To define a true class of para-differential operators, we shall not use symbols, but instead a formulation in terms of commutators with differential operators, similar to the Beals characterization of pseudo-differential operators [4]. In that way, we can give a global definition on the manifold.

If $A, P$ are two operators, we set $\operatorname{Ad}_{P} A=[P, A]$.

Definition 1.2.2 We assume given a real number $M_{0} \geq 1$. For $p \in \mathbb{N}, m \in \mathbb{R}, \nu \in \mathbb{R}_{+}$, we denote by $\widetilde{H \Psi}_{p}^{m, \nu}$ the space of p-linear maps $\mathcal{U}=\left(u_{1}, \ldots, u_{p}\right) \rightarrow A(\mathcal{U})$, defined on $C^{\infty}(X)^{p}$ with values in the space of linear maps from $C^{\infty}(X)$ to $\mathcal{D}^{\prime}(X)$, such that, for any family $\left(P_{1}, \ldots, P_{k}\right)$ of differential operators on $X$, of respective orders $d_{1}, \ldots, d_{k}$, for any $N_{1}, N_{2} \in \mathbb{N}$, there is $C>0$ and, for any $\mathcal{U}$ in $C^{\infty}(X)^{p}$, any $n^{\prime}=\left(n_{1}, \ldots, n_{p}\right) \in\left(\mathbb{N}^{*}\right)^{p}$, any $j, j^{\prime}$ in $\mathbb{N}$

$$
\begin{aligned}
\left\|\Delta_{j} \operatorname{Ad}_{P_{1}} \cdots \operatorname{Ad}_{P_{k}} A\left(\Pi_{n^{\prime}} \mathcal{U}\right) \Delta_{j^{\prime}}\right\|_{\mathcal{L}\left(L^{2}\right)} \leq C 2^{-N_{1}\left|j-j^{\prime}\right|} 2^{j\left(m+\sum_{\ell=1}^{k} d_{\ell}-k\right)}\left|n^{\prime}\right|^{\nu(p)+\nu+M_{0} k} \\
\times\left(1+2^{-j}\left|n^{\prime}\right|\right)^{-N_{2}} \prod_{\ell=1}^{p}\left\|\Pi_{n_{\ell}} u_{\ell}\right\|_{L^{2}} .
\end{aligned}
$$

If we want to make explicit in the notation the constant $M_{0}$ in the above estimate, we use the notation $\widetilde{H \Psi}_{p}^{m, \nu}\left[M_{0}\right]$ for the preceding space.

Remarks: - In comparison with (1.2.6), we see that (1.2.8) gains -1 on the order of the operator every time we make act a commutator with a $P_{k^{\prime}}$. This gain is traded against a loss on the smoothness of the coefficients $\mathcal{U}$, given by the power $M_{0} k$ of $\left|n^{\prime}\right|$. In general, $M_{0}$ will be a constant strictly larger than one. 
- It follows from the definition that $\widetilde{H \Psi}_{p}^{m-1, \nu+1} \subset \widetilde{H \Psi}_{p}^{m, \nu}$.

- If $A$ is in $\widetilde{H \Psi}_{p}^{m, \nu}$, it follows from the definition that for any $P_{1}, \ldots, P_{k}$ as in (1.2.8), any $N_{2} \in \mathbb{N}$, any $s \in \mathbb{R}$, there is $C>0$, and for any $\mathcal{U}$ in $C^{\infty}(X)^{p}$, any $n^{\prime}$ in $\left(\mathbb{N}^{*}\right)^{p}$

$$
\left|n^{\prime}\right|^{N_{2}}\left\|\operatorname{Ad}_{P_{1}} \cdots \operatorname{Ad}_{P_{k}} A\left(\Pi_{n^{\prime}} \mathcal{U}\right)\right\|_{\mathcal{L}\left(H^{s}, H^{s-m-N_{2}-\sum_{1}^{k} d_{\ell}+k}\right)} \leq C_{s, N_{2}}\left|n^{\prime}\right|^{\nu(p)+\nu+M_{0} k} \prod_{\ell=1}^{p}\left\|\Pi_{n_{\ell}} u_{\ell}\right\|_{L^{2}} .
$$

Conversely, if such an estimate holds for any $P_{1}, \ldots, P_{k}, s, N_{2}$, then $A$ satisfies (1.2.8). This equivalent characterization shows in particular that, if $\theta$ is in $C^{\infty}(X)$ and $A$ is in $\widetilde{H \Psi}_{p}^{m, \nu}$, then $\theta A$ and $A \theta$ are in $\widetilde{H \Psi}_{p}^{m, \nu}$. Moreover, estimate (1.2.9) with $N_{2}=0, k=0$ shows that if $u_{\ell}$ is in $H^{\sigma}(X)$ with $\sigma>\nu(p)+\nu+\frac{1}{2}$ for $\ell=1, \ldots, p$, then $\mathcal{U} \rightarrow A(\mathcal{U})$ extends as a continuous $p$-linear map from $H^{\sigma} \times \cdots \times H^{\sigma}$ to $\mathcal{L}\left(H^{s}, H^{s-m}\right)$ for any $s$.

- One has an inclusion

$$
\widetilde{H \Psi}_{p}^{m, \nu} \subset \widetilde{\mathcal{P}}_{p}^{m, \nu+2 M_{0}}
$$

Actually, we may write

$$
\left(\lambda_{n_{0}}^{2}-\lambda_{n_{p+1}}^{2}\right)^{2} \Pi_{n_{0}} A\left(\Pi_{n^{\prime}} \mathcal{U}\right) \Pi_{n_{p+1}}=\Pi_{n_{0}} \mathrm{Ad}_{\Delta} \operatorname{Ad}_{\Delta} A\left(\Pi_{n^{\prime}} \mathcal{U}\right) \Pi_{n_{p+1}}
$$

so that (1.2.8) implies estimates (1.2.9) with $\nu$ replaced by $\nu+2 M_{0}$.

- Note that it suffices to assume that (1.2.8) or (1.2.9) holds when the orders $d_{1}, \ldots, d_{k}$ of $P_{1}, \ldots, P_{k}$ are zero or one. Actually, any differential operator on $X$ of order $r \geq 1$ may be written as a finite linear combination with smooth coefficients of expressions $X_{1} \cdots X_{r^{\prime}}$, with $r^{\prime} \leq r$ and $X_{j}$ smooth vector field on $X$. This allows one to deduce (1.2.9) (and so (1.2.8)) for general $P_{\ell}$ 's from the estimates corresponding to operators of order zero or one.

We define from the preceding classes operators whose coefficients are given in terms of a single function $U=(u, \bar{u})$ instead of a $p$-tuple of functions.

Definition 1.2.3 Let $m \in \mathbb{R}, \nu \in \mathbb{R}_{+}, p \in \mathbb{N}$. One denotes by $H \Psi_{p}^{m, \nu}$ (resp. $\mathcal{P}_{p}^{m, \nu}$ ) the space of functions $U \rightarrow A(U)$, defined for $U=(u, \bar{u})$ in $C^{\infty}(X)$, with values in the vector space of linear maps from $C^{\infty}(X)$ to $\mathcal{D}^{\prime}(X)$, such that there is a family $A_{j}$ of elements of $\widetilde{H \Psi}_{p}^{m, \nu}$ (resp. $\widetilde{\mathcal{P}}_{p}^{m, \nu}$ ), $0 \leq j \leq p$, so that

$$
A(U)=\sum_{j=0}^{p} A_{j}(\underbrace{u, \ldots, u}_{j}, \underbrace{\bar{u}, \ldots, \bar{u}}_{p-j}) .
$$

As in the case of multi-linear operators, we see that if $\sigma>\nu(p)+\nu+\frac{1}{2}$, any element $U \rightarrow A(U)$ of $H \Psi_{p}^{m, \nu}$ or $\mathcal{P}_{p}^{m, \nu}$ extends as a continuous map from $H^{\sigma}(X)$ to $\mathcal{L}\left(H^{s}, H^{s-m}\right)$ for any $s$.

We denote by $H \Psi_{p}^{m, \nu}\left[M_{0}\right]$ the same space as above, when we want to make explicit the constant $M_{0}$ used in the definition of $\widetilde{H \Psi}_{p}^{m, \nu}\left[M_{0}\right]$. 
We may deduce from the definitions of the preceding classes composition results.

Lemma 1.2.4 Let $m, m^{\prime}$ be in $\mathbb{R}, \nu, \nu^{\prime}$ be in $\mathbb{R}_{+}, p, q$ be in $\mathbb{N}$. Assume that (1.2.4) is satisfied for some $a \leq \nu+\nu^{\prime}$. The following inclusions hold:

$$
\begin{aligned}
& \widetilde{H \Psi}_{p}^{m, \nu}\left[M_{0}\right] \circ \widetilde{H \Psi}_{q}^{m^{\prime}, \nu^{\prime}}\left[M_{0}\right] \subset \widetilde{H \Psi}_{p+q}^{m+m^{\prime}, \nu+\nu^{\prime}-a}\left[M_{0}\right] \\
& H \Psi_{p}^{m, \nu}\left[M_{0}\right] \circ H \Psi_{q}^{m^{\prime}, \nu^{\prime}}\left[M_{0}\right] \subset H \Psi_{p+q}^{m+m^{\prime}, \nu+\nu^{\prime}-a}\left[M_{0}\right]
\end{aligned}
$$

when $p>0$ and $q>0$,

$$
\begin{aligned}
& \widetilde{H \Psi}_{p}^{m, \nu}\left[M_{0}\right] \circ \widetilde{H \Psi}_{q}^{m^{\prime}, \nu^{\prime}}\left[M_{0}\right] \subset \widetilde{H \Psi}_{p+q}^{m+m^{\prime}, \nu+\nu^{\prime}}\left[M_{0}\right] \\
& H \Psi_{p}^{m, \nu}\left[M_{0}\right] \circ H \Psi_{q}^{m^{\prime}, \nu^{\prime}}\left[M_{0}\right] \subset H \Psi_{p+q}^{m+m^{\prime}, \nu+\nu^{\prime}}\left[M_{0}\right]
\end{aligned}
$$

if $p=0$ or $q=0$,

$$
\begin{aligned}
\widetilde{H \Psi}_{p}^{m, \nu}\left[M_{0}\right] \circ \widetilde{\mathcal{P}}_{q}^{m^{\prime}, \nu^{\prime}} & \subset \widetilde{\mathcal{P}}_{p+q}^{m+m^{\prime}, \nu+\nu^{\prime}-a+2 M_{0}} \\
\widetilde{\mathcal{P}}_{p}^{m, \nu} \circ \widetilde{H \Psi}_{q}^{m^{\prime}, \nu^{\prime}}\left[M_{0}\right] & \subset \widetilde{\mathcal{P}}_{p+q}^{m+m^{\prime}, \nu+\nu^{\prime}-a+2 M_{0}} \\
\widetilde{\mathcal{P}}_{p}^{m, \nu} \circ \widetilde{\mathcal{P}}_{q}^{m^{\prime}, \nu^{\prime}} & \subset \widetilde{\mathcal{P}}_{p+q}^{m+m^{\prime}, \nu+\nu^{\prime}-a}
\end{aligned}
$$

if $p>0$ and $q>0$. If $p=0$ or $q=0$, one has similar inclusions with $a=0$ in the exponents of the right hand side. Finally, (1.2.14) holds also replacing everywhere $\widetilde{H \Psi}$ (resp. $\widetilde{\mathcal{P}}$ ) by $H \Psi$ $($ resp. $\mathcal{P})$.

Proof: To prove (1.2.12), one notices that if $\mathcal{U}^{\prime}=\left(u_{1}, \ldots, u_{p}\right), \mathcal{U}^{\prime \prime}=\left(u_{p+1}, \ldots, u_{p+q}\right)$, $\operatorname{Ad}_{P_{1}} \cdots \operatorname{Ad}_{P_{K}}\left[A\left(\mathcal{U}^{\prime}\right) \circ B\left(\mathcal{U}^{\prime \prime}\right)\right]$ may be expressed from

$$
\left(\operatorname{Ad}_{P_{i_{1}}} \cdots \operatorname{Ad}_{P_{i_{\ell}}} A\left(\mathcal{U}^{\prime}\right)\right) \circ\left(\operatorname{Ad}_{P_{j_{1}}} \cdots \operatorname{Ad}_{P_{j_{\ell^{\prime}}}} B\left(\mathcal{U}^{\prime \prime}\right)\right)
$$

where $\ell+\ell^{\prime}=k,\left\{i_{1}, \ldots, i_{\ell}\right\} \cup\left\{j_{1}, \ldots, j_{\ell^{\prime}}\right\}=\{1, \ldots, k\}$. One has just to apply characterization (1.2.9) of the $\widetilde{H \Psi}$-spaces, together with (1.2.4), to obtain the first inclusion in (1.2.12), (1.2.13). The second inclusion in those formulas follows from the first one.

To prove the last inclusion (1.2.14), one writes

$$
\Pi_{n_{0}} A\left(\Pi_{n^{\prime}} \mathcal{U}^{\prime}\right) \circ B\left(\Pi_{n^{\prime \prime}} \mathcal{U}^{\prime \prime}\right) \Pi_{n_{p+1}}=\sum_{n}\left(\Pi_{n_{0}} A\left(\Pi_{n^{\prime}} \mathcal{U}^{\prime}\right) \Pi_{n}\right) \circ\left(\Pi_{n} B\left(\Pi_{n^{\prime \prime}} \mathcal{U}^{\prime \prime}\right) \Pi_{n_{p+1}}\right),
$$

and uses (1.2.5). The first and the second inclusions (1.2.5) follow from the third one and from (1.2.10). This concludes the proof.

We have defined up to now operators homogeneous of order $p$ in some function $U$. We shall need as well similar classes for which the $U$-dependence will be only $C^{1}$, with some vanishing when $U \rightarrow 0$. If $\sigma$ is a real number (that will be large enough in practice), we set for $R>0$,

$$
B_{\sigma}(R)=\left\{U \in H^{\sigma}(X) ;\|U\|_{H^{\sigma}}<R\right\} .
$$


Definition 1.2.5 Let $m \in \mathbb{R}, \nu \in \mathbb{R}_{+}, p \in \mathbb{N}, \sigma>0, R>0$. One denotes by $\Psi_{p}^{m, \nu}\left(B_{\sigma}(R)\right)$ the space of maps $U=(u, \bar{u}) \rightarrow A(U)$, defined on $B_{\sigma}(R)$, with values in the space of linear maps from $C^{\infty}(X)$ to $\mathcal{D}^{\prime}(X)$, satisfying the following conditions:

(i) For any family $P_{1}, \ldots, P_{k}$ of differential operators of orders $d_{1}, \ldots, d_{k}$ on $X$, for any $N \in \mathbb{N}$, there is $C>0$ such that, for any $U \in B_{\sigma}(R)$, any $j, j^{\prime} \in \mathbb{N}$

$$
\left\|\Delta_{j} \operatorname{Ad}_{P_{1}} \cdots \operatorname{Ad}_{P_{k}} A(U) \Delta_{j^{\prime}}\right\|_{\mathcal{L}\left(L^{2}\right)} \leq C 2^{-N\left|j-j^{\prime}\right|} 2^{j\left[m+\sum_{\ell=1}^{k} d_{\ell}-k+\left(M_{0} k+\nu(p)+\nu-\sigma\right)_{+}\right]}\|U\|_{H^{\sigma}}^{p} .
$$

(ii) For any $j, j^{\prime}, P_{1}, \ldots, P_{k}$ as above, the map $U \rightarrow \Delta_{j} \operatorname{Ad}_{P_{1}} \cdots \operatorname{Ad}_{P_{k}} A\left(\Pi_{n^{\prime}} \mathcal{U}\right) \Delta_{j^{\prime}}$ is $C^{1}$ on $B_{\sigma}(R)$ with values in $\mathcal{L}\left(L^{2}\right)$ and, for any $\sigma^{\prime}$ with $\left|\sigma^{\prime}\right| \leq \sigma$, the differential in $U$ extends as a linear map from $H^{\sigma^{\prime}}(X)$ to $\mathcal{L}\left(L^{2}\right)$ such that, for any $N \in \mathbb{N}$, there is $C>0$ so that, for any $U \in B_{\sigma}(R)$, any $j, j^{\prime} \in \mathbb{N}$, any $H$ in $H^{\sigma^{\prime}}(X)$

$$
\begin{aligned}
& \left\|\Delta_{j} \operatorname{Ad}_{P_{1}} \cdots \operatorname{Ad}_{P_{k}}\left(\partial_{U} A(U) \cdot H\right) \Delta_{j^{\prime}}\right\|_{\mathcal{L}\left(L^{2}\right)} \\
& \quad \leq C 2^{-N\left|j-j^{\prime}\right|} 2^{j\left[m+\sum_{\ell=1}^{k} d_{\ell}-k+\left(M_{0} k+\nu(p)+\nu-\sigma^{\prime}\right)_{+}\right]}\|U\|_{H^{\sigma}}^{p-1}\|H\|_{H^{\sigma^{\prime}}} .
\end{aligned}
$$

Remarks: - We may write equivalent formulations of (1.2.16), (1.2.17), asking that for any $s \in \mathbb{R}$, any $P_{1}, \ldots, P_{k}$ as in the statement of the definition, any $\sigma^{\prime}$ with $\left|\sigma^{\prime}\right| \leq \sigma$, any $U$ in $B_{\sigma}(R)$, any $H$ in $H^{\sigma^{\prime}}$,

$$
\left\|\operatorname{Ad}_{P_{1}} \cdots \operatorname{Ad}_{P_{k}} A(U)\right\|_{\mathcal{L}\left(H^{s}, H^{s-}\left(m+\sum d_{\ell}-k\right)-\left(M_{0} k+\nu(p)+\nu-\sigma\right)_{+}\right)} \leq C\|U\|_{H^{\sigma}}^{p}
$$

and

$$
\left\|\operatorname{Ad}_{P_{1}} \cdots \operatorname{Ad}_{P_{k}}\left(\partial_{U} A(U) \cdot H\right)\right\|_{\mathcal{L}\left(H^{s}, H^{s-}\left(m+\sum d_{\ell}-k\right)-\left(M_{0} k+\nu(p)+\nu-\sigma^{\prime}\right)_{+}\right)} \leq C\|U\|_{H^{\sigma}}^{p-1}\|H\|_{H^{\sigma^{\prime}}} .
$$

- In (1.2.18), (1.2.19) it would be enough to assume that these inequalities hold for all $k$ such that $M_{0} k+\nu(p)+\nu-\sigma<1$. The inequalities for larger values of $k$ will follow from the fact that $P_{\ell}$ is bounded from $H^{s}$ to $H^{s-d_{\ell}}$ for any $s$ and the assumption $M_{0} \geq 1$.

- As in the case of the preceding multi-linear classes, the space $\Psi_{p}^{m, \nu}\left(B_{\sigma}(R)\right)$ is stable by multiplication at the left or at the right by functions in $C^{\infty}(X)$. Moreover, it is enough to assume conditions $(1.2 .16),(1.2 .17)$ (or $(1.2 .18),(1.2 .19))$ for operators $P_{\ell}$ of order zero or one.

- It follows from Definition 1.2.5 and from (1.2.8) that if $q \geq p$ and if $\nu^{\prime}$ is any number with $\nu^{\prime}>\nu+\nu(q)-\nu(p)+\frac{1}{2}, H \Psi_{q}^{m, \nu}$ is contained in $\Psi_{p}^{m, \nu^{\prime}}\left(B_{\sigma}(R)\right)$ for any $\sigma>0$, any $R>0$ (using characterization (1.2.1) of Sobolev norms).

Let us state a composition result for the operators of the class we just defined, similar to Lemma 1.2.4. We shall need later on to control some semi-norms that we introduce before the statement. If $\mathcal{P}=\left(P_{1}, \ldots, P_{k}\right)$ is a collection of differential operators of orders $d_{1}, \ldots, d_{k}$, if $\sigma \in \mathbb{R}$, we set $\operatorname{Ad}_{\mathcal{P}}=\operatorname{Ad}_{P_{1}} \cdots \operatorname{Ad}_{P_{k}}$,

$$
\alpha(\mathcal{P}, p, \nu, \sigma)=\sum_{\ell=1}^{k} d_{\ell}-k+\left(M_{0} k+\nu(p)+\nu-\sigma\right)_{+} .
$$


If $A$ is in $\Psi_{p}^{m, \nu}\left(B_{\sigma}(R)\right)$ and $\sigma^{\prime}$ is in $[-\sigma, \sigma]$, we define

$$
\begin{aligned}
& \mathfrak{N}_{(\mathcal{P}, p, \nu, \sigma)}^{0, m, s}(A)=\sum_{\mathcal{P}^{\prime} \subset \mathcal{P}}\left\|\operatorname{Ad}_{\mathcal{P}^{\prime}} A(U)\right\|_{\mathcal{L}\left(H^{s}, H^{s-m-\alpha\left(\mathcal{P}^{\prime}, p, \nu, \sigma\right)}\right)} \\
& \mathfrak{N}_{\left(\mathcal{P}, p, \nu, \sigma^{\prime}\right)}^{1, m, s}(A)=\sum_{\mathcal{P}^{\prime} \subset \mathcal{P}}\|H\|_{H^{\sigma^{\prime}}} \leq 1 \\
& \operatorname{uup}\left\|\operatorname{Ad}_{\mathcal{P}^{\prime}}\left(\partial_{u} A(U) \cdot H\right)\right\|_{\mathcal{L}\left(H^{s}, H^{s-m-\alpha\left(\mathcal{P}^{\prime}, p, \nu, \sigma^{\prime}\right)}\right)}
\end{aligned}
$$

where the sums are taken over all sub-families of $\mathcal{P}$.

Lemma 1.2.6 Let $m, m^{\prime} \in \mathbb{R}, \nu, \nu^{\prime} \in \mathbb{R}_{+}, p, p^{\prime} \in \mathbb{N}, R>0$. Let $\sigma>0$ satisfying the inequality $\sigma \geq \min \left(\nu(p), \nu\left(p^{\prime}\right)\right)+\min \left(\nu, \nu^{\prime}\right)$ and let $U \rightarrow A(U)$ (resp. $U \rightarrow B(U)$ ) be in $\Psi_{p}^{m, \nu}\left(B_{\sigma}(R)\right.$ ) (resp. $\left.\Psi_{p^{\prime}}^{m^{\prime}, \nu^{\prime}}\left(B_{\sigma}(R)\right)\right)$. Then $U \rightarrow A(U) \circ B(U)$ is in $\Psi_{\max \left(p, p^{\prime}\right)}^{m+m^{\prime}, \max \left(\nu, \nu^{\prime}\right)}\left(B_{\sigma}(R)\right)$. Moreover, for any $\mathcal{P}=$ $\left(P_{1}, \ldots, P_{k}\right)$ as above, there is a constant $C>0$, depending only on $\mathcal{P}, R, p, p^{\prime}, \nu, \nu^{\prime}, m, m^{\prime}, s, \sigma^{\prime} \in$ $[-\sigma, \sigma]$ such that, if $p^{\prime \prime}=\max \left(p, p^{\prime}\right), \nu^{\prime \prime}=\max \left(\nu, \nu^{\prime}\right)$

$$
\begin{aligned}
\mathfrak{N}_{\left(\mathcal{P}, p^{\prime \prime}, \nu^{\prime \prime}, \sigma\right)}^{0, m+m^{\prime}, s}(A \circ B) & \leq C \mathfrak{N}_{(\mathcal{P}, p, \nu, \sigma)}^{0, m, s}(A) \mathfrak{N}_{\left(\mathcal{P}, p^{\prime}, \nu^{\prime}, \sigma\right)}^{0, m^{\prime}, s}(B) \\
\mathfrak{N}_{\left(\mathcal{P}, p^{\prime \prime}, \nu^{\prime \prime}, \sigma^{\prime}\right)}^{1, m+m^{\prime}, s}(A \circ B) & \leq C\left[\mathfrak{N}_{(\mathcal{P}, p, \nu, \sigma)}^{0, m, s}(A) \mathfrak{N}_{\left(\mathcal{P}, p^{\prime}, \nu^{\prime}, \sigma^{\prime}\right)}^{1, m^{\prime}}(B)+\mathfrak{N}_{\left(\mathcal{P}, p, \nu, \sigma^{\prime}\right)}^{1, m, s}(A) \mathfrak{N}_{\left(\mathcal{P}, p^{\prime}, \nu^{\prime}, \sigma\right)}^{0, m^{\prime}, s}(B)\right] .
\end{aligned}
$$

Proof: We may write $\operatorname{Ad}_{\mathcal{P}}(A(U) \circ B(U))$ as a linear combination of quantities of the form $\left(\operatorname{Ad}_{\mathcal{P}^{\prime}} A(U)\right) \circ\left(\operatorname{Ad}_{\mathcal{P}^{\prime \prime}} B(U)\right)$ where $\left(\mathcal{P}^{\prime}, \mathcal{P}^{\prime \prime}\right)$ is a partition of $\mathcal{P}$. If $k^{\prime}$ (resp. $k^{\prime \prime}$, resp. $k$ ) is the cardinal of $\mathcal{P}^{\prime}$ (resp. $\mathcal{P}^{\prime \prime}$, resp. $\mathcal{P}$ ), we shall deduce estimates (1.2.18), (1.2.19) for the composition from the corresponding estimates for each factor, if we have the inequality

$$
\alpha\left(\mathcal{P}^{\prime}, p, \nu, \sigma\right)+\alpha\left(\mathcal{P}^{\prime \prime}, p^{\prime}, \nu^{\prime}, \sigma^{\prime}\right) \leq \alpha\left(\mathcal{P}, \max \left(p, p^{\prime}\right), \max \left(\nu, \nu^{\prime}\right), \sigma^{\prime}\right)
$$

for $\left|\sigma^{\prime}\right| \leq \sigma$ (and the same inequality with $\sigma$ and $\sigma^{\prime}$ exchanged in the left hand side). This follows from

$$
\left(M_{0} k^{\prime}+\nu(p)+\nu-\sigma\right)_{+}+\left(M_{0} k^{\prime \prime}+\nu\left(p^{\prime}\right)+\nu^{\prime}-\sigma^{\prime}\right)_{+} \leq\left(M_{0} k+\max \left(\nu(p), \nu\left(p^{\prime}\right)\right)+\max \left(\nu, \nu^{\prime}\right)-\sigma^{\prime}\right)_{+}
$$

which is a consequence of the assumptions on $\sigma, \sigma^{\prime}$. Inequalities (1.2.22) follow from the proof.

To state a more general version of Theorem 1.1.1, we introduce classes of functions defined on $B_{\sigma}(R)$, in terms of the preceding classes of operators.

Definition 1.2.7 Let $m \in \mathbb{R}, \nu \in \mathbb{R}_{+}, p \in \mathbb{N}, \sigma \in \mathbb{R}, R>0$. Assume

$$
\sigma>\nu(p)+\nu+\frac{1}{2}, 2 \sigma \geq m
$$

(i) One denotes by $H_{\mathcal{F}_{p, \mathrm{E}}^{m, \nu}}\left(B_{\sigma}(R)\right)$ (resp. $H \mathcal{F}_{p, \mathrm{H}}^{m, \nu}\left(B_{\sigma}(R)\right)$, resp. $\left.H \mathcal{F}_{p}^{m, \nu}\left(B_{\sigma}(R)\right)\right)$ the space of functions $F$ defined on $B_{\sigma}(R)$, real valued, that may be written, for some element $A_{p}$ of $H \Psi_{p}^{m, \nu}$ as

$$
F(U)=\operatorname{Re} \int_{X}\left[A_{p}(U) u\right] u d \mu
$$


resp. as

$$
F(U)=\operatorname{Re} \int_{X}\left[A_{p}(U) u\right] \bar{u} d \mu
$$

resp. as the sum of an element of $H \mathcal{F}_{p, \mathrm{E}}^{m, \nu}\left(B_{\sigma}(R)\right)$ and of an element of $H \mathcal{F}_{p, \mathrm{H}}^{m, \nu}\left(B_{\sigma}(R)\right)$. One uses the notation $H \mathcal{F}_{p}^{m, \nu}\left(B_{\sigma}(R)\right)\left[M_{0}\right]$ when one wants to keep track of the constant $M_{0}$ as in the definition of $H \Psi_{p}^{m, \nu}\left[M_{0}\right]$.

(ii) One denotes by $\mathcal{F}_{p, \mathrm{E}}^{m, \nu}\left(B_{\sigma}(R)\right)$ (resp. $\mathcal{F}_{p, \mathrm{H}}^{m, \nu}\left(B_{\sigma}(R)\right)$, resp. $\mathcal{F}_{p}^{m, \nu}\left(B_{\sigma}(R)\right)$ ) the space of functions defined on $B_{\sigma}(R)$ by similar expressions as above, but with $A_{p}$ in $\Psi_{p}^{m, \nu}\left(B_{\sigma}(R)\right)$.

(iii) One denotes by $\mathcal{G}_{p}^{m, \nu}\left(B_{\sigma}(R)\right)$ the space of functions defined on $B_{\sigma}(R)$ as $\mathcal{F}_{p}^{m, \nu}\left(B_{\sigma}(R)\right)$, but with $A_{p}$ in $\mathcal{P}_{p}^{m, \nu}$.

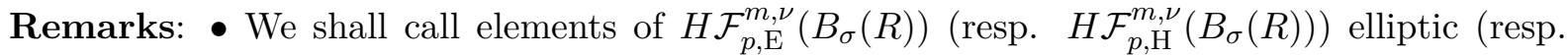
hyperbolic) elements.

- Since $A_{p}(U)$ sends $H^{\sigma}$ to $H^{\sigma-m}$, the second assumption (1.2.23) shows that (1.2.24), (1.2.25) make sense.

- It follows from Definition 1.2 .3 and inequality (1.2.7) that if $F$ is in $\mathcal{G}_{p}^{m, \nu}\left(B_{\sigma}(R)\right)$ and $\sigma$ is large enough relatively to $m, \nu$, then $\nabla F$ is in $H^{\sigma-m}$.

Let us define the set $\mathcal{N}$ of exceptional values of the mass that have to be excluded. For $\mathbf{m} \in$ ] $0,+\infty\left[\right.$, define if $p \in \mathbb{N}, 0 \leq \ell \leq p, \xi_{j} \in \mathbb{R}$ for $0 \leq j \leq p+1$,

$$
\begin{aligned}
F_{\mathbf{m}}^{p, \ell}\left(\xi_{0}, \ldots, \xi_{p+1}\right) & =\sum_{j=0}^{\ell} \sqrt{\mathbf{m}^{2}+\xi_{j}^{2}}-\sum_{j=\ell+1}^{p+1} \sqrt{\mathbf{m}^{2}+\xi_{j}^{2}} \\
G_{\mathbf{m}}^{p, \ell}\left(\xi_{1}, \ldots, \xi_{p}\right) & =\sum_{j=1}^{\ell} \sqrt{\mathbf{m}^{2}+\xi_{j}^{2}}-\sum_{j=\ell+1}^{p} \sqrt{\mathbf{m}^{2}+\xi_{j}^{2}} .
\end{aligned}
$$

Denote by $Z^{\ell}(p)\left(\right.$ resp. $\left.\underline{Z}^{\ell}(p)\right)$ the set of those $\left(n_{0}, \ldots, n_{p+1}\right) \in\left(\mathbb{N}^{*}\right)^{p+2}\left(\right.$ resp. $\left(n_{1}, \ldots, n_{p}\right) \in$ $\left.\left(\mathbb{N}^{*}\right)^{p}\right)$ such that there is a bijection $\tau:\{0, \ldots, \ell\} \rightarrow\{\ell+1, \ldots, p+1\}$ (resp. $\tau:\{1, \ldots, \ell\} \rightarrow$ $\{\ell+1, \ldots, p\}$ ) with $n_{\tau(j)}=n_{j}$ for any $j=0, \ldots, \ell$ (resp. $j=1, \ldots, \ell$ ). Of course, these sets are empty if $p$ is odd, or if $p$ is even and $\ell \neq p / 2$. Then, Theorem 4.7 of [8] (see also, for similar results in one dimension, Bambusi [1] and Bourgain [5]) asserts that there is $\mathcal{N} \subset] 0,+\infty$ [ of zero measure and for any $p \in \mathbb{N}, L_{0}>0$ such that, for any $\left.\mathbf{m} \in\right] 0,+\infty[-\mathcal{N}$, there is $c>0$ with

$$
\begin{array}{r}
\left|F_{\mathbf{m}}^{p, \ell}\left(\lambda_{n_{0}}, \ldots, \lambda_{n_{p+1}}\right)\right| \geq c\left(n_{0}+\cdots+n_{p+1}\right)^{-L_{0}} \\
\inf _{\alpha \in \mathbb{Z}}\left|G_{\mathbf{m}}^{p, \ell}\left(\lambda_{n_{1}}, \ldots, \lambda_{n_{p}}\right)+\frac{\alpha}{2}\right| \geq c\left(n_{1}+\cdots+n_{p}\right)^{-L_{0}}
\end{array}
$$

for any $\left(n_{0}, \ldots, n_{p+1}\right) \in\left(\mathbb{N}^{*}\right)^{p+2}-Z^{\ell}(p)$ and any $\left(n_{1}, \ldots, n_{p}\right) \in\left(\mathbb{N}^{*}\right)^{p}-\underline{Z}^{\ell}(p)$ respectively.

We may state a more general version of Theorem 1.1.1. 
Theorem 1.2.8 Let $P \in \mathbb{N}^{*}$ be given, $R>0, \nu^{\prime} \in \mathbb{R}_{+}$. Let $P^{\prime} \in \mathbb{N}^{*}, \sigma>\nu\left(P^{\prime}\right)+\nu+\frac{1}{2}$ and let

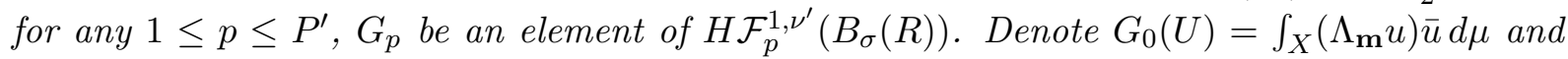
$G(U)=\sum_{p=0}^{P^{\prime}} G_{p}(U)$. Then for any $\left.\mathbf{m} \in\right] 0,+\infty\left[-\mathcal{N}\right.$, there is $s_{0}>0$ such that, for any $s>s_{0}$, there are $\left.\epsilon_{0} \in\right] 0,1[, c>0, C>0$ and, for any $\epsilon \in] 0, \epsilon_{0}\left[\right.$, for any $u_{0}$ in the unit ball of $H^{s}(X)$, there is a unique solution $u$ in $C^{0}(]-T_{\epsilon}, T_{\epsilon}\left[, H^{s}(X)\right)$ of the equation

$$
u_{t}=i \nabla_{\bar{u}} G(u, \bar{u}), u(0, x)=\epsilon u_{0}(x)
$$

with $T_{\epsilon} \geq c \epsilon^{-P}$. Moreover

$$
\sup _{|t|<T_{\epsilon}}\|u(t, \cdot)\|_{H^{s}} \leq C \epsilon
$$

In the rest of this subsection, we shall prove that Theorem 1.2.8 contains Theorem 1.1.1. We do need the more general statement of Theorem 1.2.8 because the structure of $G=\sum_{p} G_{p}$ will be stable along the proof.

We have seen that the proof of Theorem 1.1.1 may be reduced to solving equation (1.1.18) with a Hamiltonian $G$ given by (1.1.11), with $V$ related to $U=(u, \bar{u})$ by (1.1.13). Consequently, Theorem 1.1.1 follows from Theorem 1.2.8 and the following lemma:

Lemma 1.2.9 Set $G(U)=G_{0}(U)-F\left(\Lambda_{\mathbf{m}}^{-1 / 2} \frac{\sqrt{2}}{2 i}(u-\bar{u})\right)$. Then $G$ has the structure of the assumption of Theorem 1.2.8 for any fixed $R>0$ if $\sigma>\nu(p)+\nu+\frac{1}{2}, \nu^{\prime} \geq \frac{d}{2}+1$ and $\nu(p) \geq p\left(\frac{d}{2}+1\right)$ for any $p=1, \ldots, P^{\prime}$.

Before giving the proof of the lemma, we shall establish some properties of $F(v)=\int_{X} f(v, d v) d \mu$, where $(z, \rho) \rightarrow f(z, \rho)$ is a smooth function on $\mathbb{R} \times T^{*} X$, polynomial in $z$ and in the fiber variable of $\rho$, and $v$ is in $\operatorname{Lip}(X)$, the space of real valued lipschitz function on $X$. We denote for a while by $\{\cdot, \cdot\}$ the Poisson bracket between functions on $T^{*} X$, as at the beginning of subsection 1.1. We consider systematically any smooth enough function on $X$ as a function on $T^{*} X$, independent of the fiber variable. For $h$ a given element in $\operatorname{Lip}(X), f: \mathbb{R} \times T^{*} X \rightarrow \mathbb{R}$ a smooth function, we define a new function $\mathcal{D}(h) f$ from $\mathbb{R} \times T^{*} X$ to $\mathbb{R}$ by the formula

$$
\mathcal{D}(h) f=\frac{\partial f}{\partial z} h-\{h, f\} .
$$

This new function is no longer smooth in $\rho \in T^{*} X$, because of the limited smoothness of $h$. Nevertheless, a composition $\mathcal{D}\left(h_{1}\right) \cdots \mathcal{D}\left(h_{p}\right) f$ is still meaningful, for any $h_{1}, \ldots, h_{N} \in \operatorname{Lip}(X)$ : for instance

$$
\mathcal{D}\left(h_{1}\right) \mathcal{D}\left(h_{2}\right) f=\frac{\partial^{2} f}{\partial z^{2}} h_{1} h_{2}-\left\{h_{1}, \frac{\partial f}{\partial z}\right\} h_{2}-\left\{h_{2}, \frac{\partial f}{\partial z}\right\} h_{1}+\left\{h_{2},\left\{h_{1}, f\right\}\right\}
$$

never involves more than one derivative acting on each $h_{j}$. By an immediate computation in local coordinates, one checks that $F(U)=\int_{X} f(v, d v) d \mu$ is a smooth function on $\operatorname{Lip}(X)$, whose differentials are given by

$$
d^{p} F(v) \cdot\left(h_{1}, \ldots, h_{p}\right)=\int_{X}\left(\prod_{j=1}^{p} \mathcal{D}\left(h_{j}\right) f\right)(v, d v) d \mu .
$$


This formula, and the above expression for $\mathcal{D}\left(h_{j}\right)$, shows that when $v$ stays in a fixed ball of $\operatorname{Lip}(X)$,

$$
\left|d^{p} F(v) \cdot\left(h_{1}, \ldots, h_{p}\right)\right| \leq C \prod_{j=3}^{p}\left\|h_{j}\right\|_{\operatorname{Lip}(X)}\left\|h_{1}\right\|_{H^{1}}\left\|h_{2}\right\|_{H^{1}} .
$$

We shall need bounds on the commutator between the symmetric operator associated to the bilinear form $\left(h_{1}, h_{2}\right) \rightarrow d^{2} F(v)\left(h_{1}, h_{2}\right)$ and differential operators. We fix some notation: if $w_{1}, \ldots, w_{p}$ are smooth functions on $X$, if $k \in \mathbb{N}$, we set

$$
M_{k}\left(w_{1}, \ldots, w_{p}\right)=\sum_{\left|\alpha_{1}\right|+\cdots+\left|\alpha_{p}\right|=k} \prod_{j=1}^{p}\left\|\partial^{\alpha} w_{j}\right\|_{\operatorname{Lip}(X)} .
$$

Definition 1.2.10 Let $p \in \mathbb{N}, r \in \mathbb{N}, m \in \mathbb{Z}$. If $m<0$, set $\widetilde{H \mathcal{A}}_{p, r}^{m}=\{0\}$. If $m \geq 0$, we denote by $\widetilde{H \mathcal{A}}_{p, r}^{m}$ the space of $p$-linear maps $\left(v_{1}, \ldots, v_{p}\right) \rightarrow A\left(v_{1}, \ldots, v_{p}\right)$, defined on $C^{\infty}(M, \mathbb{R})^{p}$, with values in the space symmetric bilinear forms on $C^{\infty}(X, \mathbb{R}) \times C^{\infty}(X, \mathbb{R})$, satisfying the following condition: for any local chart $\theta: V \rightarrow U$ from an open subset $V$ of $X$ to an open subset $U$ of $\mathbb{R}^{d}$, for any $\gamma_{1}, \gamma_{2}$ in $\mathbb{N}^{d}$ with $\left|\gamma_{1}\right|+\left|\gamma_{2}\right| \leq m$, there is a map

$$
\begin{aligned}
a_{\gamma_{1}, \gamma_{2}}: & \left(x, v_{1}, \ldots, v_{p}\right) \longrightarrow a_{\gamma_{1}, \gamma_{2}}\left(x, v_{1}, \ldots, v_{p}\right) \\
& U \times C^{\infty}(M, \mathbb{R})^{p} \rightarrow \mathbb{R}
\end{aligned}
$$

which is $p$-linear in $\left(v_{1}, \ldots, v_{p}\right)$, smooth in $x$, satisfying for any $\alpha \in \mathbb{N}^{d}$

$$
\left|\partial_{x}^{\alpha} a_{\gamma_{1}, \gamma_{2}}\left(x, v_{1}, \ldots, v_{p}\right)\right| \leq C M_{|\alpha|+r}\left(v_{1}, \ldots, v_{p}\right)
$$

such that, for any $h_{1}, h_{2}$ in $C_{0}^{\infty}(V, \mathbb{R})$,

$$
A\left(v_{1}, \ldots, v_{p}\right)\left(h_{1}, h_{2}\right)=\sum_{\left|\gamma_{1}\right|+\left|\gamma_{2}\right| \leq m} \int_{U} a_{\gamma_{1}, \gamma_{2}}\left(x, v_{1}, \ldots, v_{p}\right)\left(\partial_{x}^{\gamma_{1}}\left[h_{1} \circ \theta^{-1}\right]\right)\left(\partial_{x}^{\gamma_{2}}\left[h_{2} \circ \theta^{-1}\right]\right) d x .
$$

One denotes by $H \mathcal{A}_{p, r}^{m}$ the space of maps $v \rightarrow A(v)$ that may be written $\tilde{A}(v, \ldots, v)$ for some $\tilde{A}$ in $\widetilde{H \mathcal{A}}_{p, r}^{m}$.

We shall use the following lemma:

Lemma 1.2.11 Let $P$ be a differential operator of order $\ell$ on $X, A$ be an element of $\widetilde{H \mathcal{A}_{p, r}}$. Then $\left(h_{1}, h_{2}\right) \rightarrow A\left(v_{1}, \ldots, v_{p}\right)\left(P h_{1}, h_{2}\right)-A\left(v_{1}, \ldots, v_{p}\right)\left(h_{1},{ }^{t} P h_{2}\right)$ is in $\sum_{\ell^{\prime}=1}^{\ell} \widetilde{H \mathcal{A}} \widetilde{A}_{p, r+\ell^{\prime}}^{m+\ell-\ell^{\prime}}$.

Proof: If $\tilde{A}$ is in $\widetilde{H \mathcal{A}}_{p, r}^{m}$, we may write, according to (1.2.36), the quantity under study from expressions

$$
\begin{aligned}
& \int_{U} a_{\gamma_{1}, \gamma_{2}}\left(x, v_{1}, \ldots, v_{p}\right)\left(\partial_{x}^{\gamma_{1}}\left[P h_{1} \circ \theta^{-1}\right]\right)\left(\partial_{x}^{\gamma_{2}}\left[h_{2} \circ \theta^{-1}\right]\right) d x \\
& -\int_{U} a_{\gamma_{1}, \gamma_{2}}\left(x, v_{1}, \ldots, v_{p}\right)\left(\partial_{x}^{\gamma_{1}}\left[h_{1} \circ \theta^{-1}\right]\right)\left(\partial_{x}^{\gamma_{2}}\left[\left({ }^{t} P h_{2}\right) \circ \theta^{-1}\right]\right) d x .
\end{aligned}
$$


If we perform integrations by parts in the second integral, we get expressions involving at most $\ell^{\prime}$ derivatives of $a_{\gamma_{1}, \gamma_{2}}$ and $\left|\gamma_{1}\right|+\left|\gamma_{2}\right|-\ell^{\prime}+\ell$ derivatives of $\left(h_{1}, h_{2}\right)$ for $1 \leq \ell^{\prime} \leq \ell$. This shows that we obtain an element of $\sum_{\ell^{\prime}=1}^{\ell} \widetilde{H \mathcal{A}_{p, r+\ell^{\prime}}^{m+\ell}}$.

Proof of Lemma 1.2.9: We denote by $Q$ the pseudo-differential operator of order $-1 / 2, Q=$ $\Lambda_{\mathbf{m}}^{-1 / 2} \frac{\sqrt{2}}{2 i}[1-1]$, acting on complex valued functions $U=\left[\frac{u}{u}\right]$. The Hamiltonian $G$ of the statement of the lemma is expressed in terms of

$$
U \rightarrow F(Q U)=\int_{X} f(Q U, d Q U) d \mu .
$$

Using notation $(1.2 .2)$, with the convention $S_{-1}=0$, we write $\operatorname{Id}=\sum_{j=0}^{+\infty}\left(S_{j}-S_{j-1}\right)$ so that

$$
F(Q U)=\sum_{j=0}^{+\infty}\left(F\left(Q S_{j} U\right)-F\left(Q S_{j-1} U\right)\right)=\sum_{j=0}^{+\infty} \int_{0}^{1} d F\left(Q S_{j}(t) U\right) \cdot Q \Delta_{j} U d t
$$

where $S_{j}(t)=t S_{j}+(1-t) S_{j-1}$. Applying the same decomposition to $U \rightarrow d F\left(Q S_{j}(t) U\right)$, which is possible since $d F(0)=0$, we get

$$
F(U)=\sum_{j=0}^{+\infty} \sum_{j^{\prime}=0}^{+\infty} \int_{0}^{1} \int_{0}^{1} d^{2} F\left(Q S_{j j^{\prime}}\left(t, t^{\prime}\right) U\right) \cdot\left(Q \Delta_{j} U, Q S_{j}(t) \Delta_{j^{\prime}} U\right) d t d t^{\prime}
$$

with $S_{j j^{\prime}}\left(t, t^{\prime}\right)=S_{j}(t) S_{j^{\prime}}\left(t^{\prime}\right)$. We use Taylor formula to decompose

$$
d^{2} F(W)=\sum_{p=0}^{+\infty} \frac{1}{p !} d^{p+2} F(0) \cdot W^{p}
$$

where the sum is finite since $F$ is polynomial. Plugging this expression in (1.2.37) and using the expression (1.2.31) of $d^{p} F$, and the fact that $F$ vanishes at least at order three at zero, we may write

$$
F(Q U)=\sum_{p=1}^{+\infty} A_{p}(U) \cdot(U, U)
$$

where the symmetric bilinear forms $A_{p}(U)$ are given by

$$
A_{p}(U)=\sum_{j_{1}=0}^{+\infty} \sum_{j_{1}^{\prime}=0}^{+\infty} \int_{0}^{1} \int_{0}^{1} A_{p}^{j_{1}, j_{1}^{\prime}}\left(U, t, t^{\prime}\right) d t d t^{\prime}, p \geq 1
$$

denoting

$$
A_{p}^{j_{1}, j_{1}^{\prime}}\left(U, t, t^{\prime}\right) \cdot\left(H_{1}, H_{2}\right)=\left.\frac{1}{p !} \int_{X} \mathcal{D}\left(Q \Delta_{j_{1}} H_{1}\right) \mathcal{D}\left(Q S_{j_{1}}(t) \Delta_{j_{1}^{\prime}} H_{2}\right) g_{p}(U, \cdot)\right|_{\{0\} \times X} d \mu
$$

with

$$
g_{p}(U, z, \rho)=\left[\mathcal{D}\left(Q S_{j_{1} j_{1}^{\prime}}\left(t, t^{\prime}\right) U\right)\right]^{p} f(z, \rho)
$$


(The restriction in the right hand side of (1.2.40) means restriction to the zero section $\{0\} \times X$ of $\left.\mathbb{R} \times T^{*} X\right)$.

To finish the proof of the lemma, we have to show that $G_{p}(U)=-A_{p}(U) \cdot(U, U)$ is in $H \mathcal{F}_{p}^{1, \nu^{\prime}}\left(B_{\sigma}(R)\right)$ with $\sigma>\nu(p)+\nu^{\prime}+\frac{1}{2}$ for any $p \geq 1$. This follows from next lemma.

Lemma 1.2.12 For $\mathcal{U}=\left(U_{1}, \ldots, U_{p}\right)$ a family of $C^{\infty}(X)$ functions, set

$$
\tilde{g}_{p}\left(\mathcal{U}, t, t^{\prime}, z, \rho\right)=\prod_{\ell=1}^{p} \mathcal{D}\left(Q S_{j_{1} j_{1}^{\prime}}\left(t, t^{\prime}\right) U_{\ell}\right) \cdot f(z, \rho)
$$

and define $A_{p}^{j_{1}, j_{1}^{\prime}}\left(\mathcal{U}, t, t^{\prime}\right)$ (resp. $\left.A_{p}(\mathcal{U})\right)$ by (1.2.40) (resp. (1.2.39)) with $g_{p}$ replaced by $\tilde{g}_{p}$ and with $U$ replaced by $\mathcal{U}$. Then, if we identify these symmetric bilinear forms on $C^{\infty}(X) \times C^{\infty}(X)$ to the corresponding linear maps from $C^{\infty}(X)$ to $\mathcal{D}^{\prime}(X), A_{p}(\mathcal{U})$ belongs to $\widetilde{H \Psi}_{p}^{1, \nu^{\prime}}$ if $\nu^{\prime} \geq \nu\left(\frac{d}{2}+1\right)$ and $\nu(p) \geq p\left(\frac{d}{2}+1\right)$.

Proof: Define for $p \in \mathbb{N}^{*}$

$$
B_{p}^{j_{1}, j_{1}^{\prime}}\left(\mathcal{U}, t, t^{\prime}\right)\left(H_{1}, H_{2}\right)=\left.\frac{1}{p !} \int_{X} \mathcal{D}\left(H_{1}\right) \mathcal{D}\left(H_{2}\right) \tilde{g}_{p}(\mathcal{U}, \cdot)\right|_{\{0\} \times X} d \mu .
$$

Let us check that $B_{p}^{j_{1} j_{1}^{\prime}}$ is in $\widetilde{H \mathcal{A}}_{p, 0}^{2}$, with constants in estimates (1.2.35) uniform in $j_{1}, j_{1}^{\prime}, t, t^{\prime}$.

It follows from the definition of $\mathcal{D}(h)$ that $\left|\mathcal{D}\left(h_{1}\right) \cdots \mathcal{D}\left(h_{p}\right) f\right|$ is bounded from above by a constant times $C \prod_{1}^{p}\left\|h_{j}\right\|_{\operatorname{Lip}(X)}$. Expression (1.2.42), and boundedness of pseudo-differential operators of order $-1 / 2$ like $Q S_{j_{1}, j_{1}^{\prime}}\left(t, t^{\prime}\right)$ on $\operatorname{Lip}(X)$, show that $\partial_{x}^{\alpha}$ derivatives of $\tilde{g}_{p}\left(\mathcal{U}, t, t^{\prime}, z, \rho\right)$ are bounded, when $(z, \rho)$ stays in a compact set, in terms of $M_{|\alpha|}\left(U_{1}, \ldots, U_{p}\right)$ defined by (1.2.33). If in (1.2.43), $H_{1}, H_{2}$ are supported in a chart domain, it follows that $B_{p}^{j_{1}, j_{1}^{\prime}}\left(\mathcal{U}, t, t^{\prime}\right)\left(H_{1}, H_{2}\right)$ is given by an expression of form (1.2.36) with $m=2$, and with $\partial_{x}^{\alpha}$-derivatives of the coefficients $a_{\gamma_{1}, \gamma_{2}}$ bounded by the right hand side of $(1.2 .35)$ with $r=0$. This shows that $B_{p}^{j_{1}, j_{1}^{\prime}}\left(\mathcal{U}, t, t^{\prime}\right)$ is in $\widetilde{H \mathcal{A}}_{p, 0}^{2}$, uniformly in $j_{1}, j_{1}^{\prime}, t, t^{\prime}$. Moreover, because of the definition of $\tilde{g}_{p}, B_{p}^{j_{1}, j_{1}^{\prime}}\left(\Pi_{n^{\prime}} \mathcal{U}, t, t^{\prime}\right) \equiv 0$ if $\left|n^{\prime}\right| \geq C 2^{\min \left(j_{1}, j_{1}^{\prime}\right)}$ for some large enough constant $C$.

We have to deduce from these informations on $B_{p}^{j_{1}, j_{1}^{\prime}}$ the conclusion of the lemma for $A_{p}(\mathcal{U})$. Identifying bilinear forms and linear maps, we deduce from (1.2.40), (1.2.42), (1.2.43) that $A_{p}^{j_{1}, j_{1}^{\prime}}$ may be written in terms of $B_{p}^{j_{1}, j_{1}^{\prime}}$ by

$$
A_{p}^{j_{1}, j_{1}^{\prime}}\left(\mathcal{U}, t, t^{\prime}\right)=\Delta_{j_{1}}{ }^{t} Q B_{p}^{j_{1}, j_{1}^{\prime}}\left(\mathcal{U}, t, t^{\prime}\right) Q S_{j_{1}}(t) \Delta_{j_{1}^{\prime}}
$$

Let $\mathcal{P}=\left(P_{1}, \ldots, P_{k}\right)$ be a family of differential operators, and denote $\operatorname{Ad}_{\mathcal{P}}=\operatorname{Ad}_{P_{1}} \cdots \operatorname{Ad}_{P_{k}}$. By definition of $A_{p}(\mathcal{U})$ and $(1.2 .44)$

$$
\begin{aligned}
& \Delta_{j} \operatorname{Ad}_{\mathcal{P}} A_{p}\left(\Pi_{n^{\prime}} \mathcal{U}\right) \Delta_{j^{\prime}}= \\
& \quad \sum_{j_{1}} \sum_{j_{1}^{\prime}} \int_{0}^{1} \int_{0}^{1} \Delta_{j} \operatorname{Ad}_{\mathcal{P}}\left[\Delta_{j_{1}}{ }^{t} Q B_{p}^{j_{1}, j_{1}^{\prime}}\left(\Pi_{n^{\prime}} \mathcal{U}, t, t^{\prime}\right) Q S_{j_{1}}(t) \Delta_{j_{1}^{\prime}}\right] \Delta_{j^{\prime}} d t d t^{\prime} .
\end{aligned}
$$


The integrand may be written from a sum of terms

$$
\Delta_{j} \operatorname{Ad}_{\mathcal{P}^{\prime}}\left[\Delta_{j_{1}}{ }^{t} Q\right] \cdot \operatorname{Ad}_{\mathcal{P}^{\prime \prime}}\left[B_{p}^{j_{1}, j_{1}^{\prime}}\left(\Pi_{n^{\prime}} \mathcal{U}, t, t^{\prime}\right)\right] \cdot \operatorname{Ad}_{\mathcal{P}^{\prime \prime \prime}}\left[Q S_{j_{1}}(t) \Delta_{j_{1}^{\prime}}\right] \Delta_{j^{\prime}}
$$

where $\mathcal{P}^{\prime} \cup \mathcal{P}^{\prime \prime} \cup \mathcal{P}^{\prime \prime \prime}$ is a partition of $\mathcal{P}$. Let $\tilde{\varphi} \in C_{0}^{\infty}(\mathbb{R}-\{0\})$ with $\tilde{\varphi} \varphi=\varphi$, and denote by $\widetilde{\Delta}_{j}=\tilde{\varphi}\left(2^{-j} \sqrt{-\Delta}\right)$ the corresponding spectral cut-off (with the obvious modification for $j=0$ ). We insert $\widetilde{\Delta}_{j_{2}} \Delta_{j_{2}}, \widetilde{\Delta}_{j_{2}^{\prime}} \Delta_{j_{2}^{\prime}}$ in (1.2.46) and rewrite this expression as

$$
\sum_{j_{2}} \sum_{j_{2}^{\prime}} K\left(j, j_{1}, j_{2}\right) \Delta_{j_{2}} \operatorname{Ad}_{\mathcal{P}^{\prime \prime}}\left[B_{p}^{j_{1}, j_{1}^{\prime}}\left(\Pi_{n^{\prime}} \mathcal{U}, t, t^{\prime}\right)\right] \Delta_{j_{2}^{\prime}} K^{\prime}\left(j_{2}^{\prime}, j_{1}^{\prime}, j^{\prime}\right)
$$

with

$$
K\left(j, j_{1}, j_{2}\right)=\Delta_{j} \operatorname{Ad}_{\mathcal{P}^{\prime}}\left[\Delta_{j_{1}}{ }^{t} Q\right] \widetilde{\Delta}_{j_{2}}, K^{\prime}\left(j_{2}^{\prime}, j_{1}^{\prime}, j^{\prime}\right)=\widetilde{\Delta}_{j_{2}^{\prime}} \operatorname{Ad}_{\mathcal{P}^{\prime \prime \prime}}\left[Q S_{j_{1}}(t) \Delta_{j_{1}^{\prime}}\right] \Delta_{j^{\prime}} .
$$

By Proposition A.1 of the appendix, for any $N \in \mathbb{N}$, there is $C_{N}>0$ such that

$$
\begin{aligned}
\left\|K\left(j, j_{1}, j_{2}\right)\right\|_{\mathcal{L}\left(L^{2}\right)} & \leq C_{N} 2^{-N\left[\left|j-j_{1}\right|+\left|j_{1}-j_{2}\right|\right]-\frac{j}{2}+j D} \\
\left\|K^{\prime}\left(j_{2}^{\prime}, j_{1}^{\prime}, j^{\prime}\right)\right\|_{\mathcal{L}\left(L^{2}\right)} & \leq C_{N} 2^{-N\left[\left|j^{\prime}-j_{1}^{\prime}\right|+\left|j_{1}^{\prime}-j_{2}^{\prime}\right|\right]-\frac{j^{\prime}}{2}+j^{\prime} D^{\prime}}
\end{aligned}
$$

with $D=\sum_{P \in \mathcal{P}^{\prime}} \operatorname{deg}(P)-\left|\mathcal{P}^{\prime}\right|, D^{\prime}=\sum_{P \in \mathcal{P}^{\prime \prime \prime}} \operatorname{deg}(P)-\left|\mathcal{P}^{\prime \prime \prime}\right|$. These estimates, and an elementary computation, show that (1.2.45) will satisfy (1.2.8) with $m=1, M_{0}=1$ if we may prove that for any $N_{1} \in \mathbb{N}$, there is $N_{1}^{\prime}$ such that

$$
\begin{array}{r}
\left\|\Delta_{j_{2}} \operatorname{Ad}_{\mathcal{P}^{\prime \prime}}\left[B_{p}^{j_{1} j_{1}^{\prime}}\left(\Pi_{n^{\prime}} \mathcal{U}, t, t^{\prime}\right)\right] \Delta_{j_{2}^{\prime}}\right\|_{\mathcal{L}\left(L^{2}\right)} \leq C 2^{-N_{1}\left|j_{2}-j_{2}^{\prime}\right|+N_{1}^{\prime}\left(\left|j_{1}-j_{2}\right|+\left|j_{1}^{\prime}-j_{2}^{\prime}\right|\right)} 2^{j_{2}\left[2+D^{\prime \prime}\right]} \\
\times\left|n^{\prime}\right|^{\nu(p)+\left|\mathcal{P}^{\prime \prime}\right|}\left(1+2^{-j_{1}}\left|n^{\prime}\right|\right)^{-N_{2}} \prod\left\|\Pi_{n_{\ell}} u_{\ell}\right\|_{L^{2}},
\end{array}
$$

where $D^{\prime \prime}=\sum_{P \in \mathcal{P}^{\prime \prime}} \operatorname{deg}(P)-\left|\mathcal{P}^{\prime \prime}\right|$. Note that a commutator $\left[P, B_{p}^{j_{1}, j_{1}^{\prime}}\left(\Pi_{n^{\prime}} \mathcal{U}, t, t^{\prime}\right)\right]$ is the translation at the level of linear maps of the quantities studied in Lemma 1.2.11 at the level of bilinear forms. By this lemma, $\operatorname{Ad}_{\mathcal{P}^{\prime \prime}} B_{p}^{j_{1}, j_{1}^{\prime}}\left(\Pi_{n^{\prime}} \mathcal{U}, t, t^{\prime}\right)$ will be the sum indexed by $a$ in $\left\{0, \ldots, D^{\prime \prime}\right\}$ of elements of $\widetilde{H \mathcal{A}} D_{p,\left|\mathcal{P}^{\prime \prime}\right|+a}$. Expressions (1.2.36) and bounds (1.2.35) imply that the left hand side of (1.2.47) is smaller than

$$
C M_{\left|\mathcal{P}^{\prime \prime}\right|+a}\left(\Pi_{n^{\prime}} \mathcal{U}\right) \sum_{\left|\gamma_{2}\right|+\left|\gamma_{2}^{\prime}\right| \leq D^{\prime \prime}-a+2} 2^{j_{2}\left|\gamma_{2}\right|+j_{2}^{\prime}\left|\gamma_{2}^{\prime}\right|} .
$$

By (1.2.33) and Sobolev injections, this is smaller than

$$
C\left|n^{\prime}\right|^{\left|\mathcal{P}^{\prime \prime}\right|+p\left[\frac{d}{2}+1\right]} 2^{j_{2}\left(D^{\prime \prime}+2\right)+\left(D^{\prime \prime}+2-a\right)\left|j_{2}-j_{2}^{\prime}\right|}\left(\left|n^{\prime}\right| 2^{-j_{2}}\right)^{a} \prod_{1}^{p}\left\|\Pi_{n_{\ell}} u_{\ell}\right\|_{L^{2}} .
$$

We have seen that if $B_{p}^{j_{1}, j_{1}^{\prime}}\left(\Pi_{n^{\prime}} \mathcal{U}, t, t^{\prime}\right) \neq 0$, then $\left|n^{\prime}\right| \leq C \min \left(2^{j_{1}}, 2^{j_{1}^{\prime}}\right)$. This shows that (1.2.48) implies (1.2.47) with $\nu(p)=p\left[\frac{d}{2}+1\right]$ if we are able to show that we may improve (1.2.48) by a factor $2^{N_{1}\left[\left|j_{1}-j_{2}\right|+\left|j_{1}^{\prime}-j_{2}^{\prime}\right|-\left|j_{2}-j_{2}^{\prime}\right|\right]}$ for any $N_{1}$. To do so, we denote by $\bar{\Delta}_{j}$ a new cut-off such that, for $j>0, \Delta \bar{\Delta}_{j}=2^{2 j} \Delta_{j}$. When $0<j_{2}^{\prime} \leq j_{2}$, write

$$
\begin{aligned}
2^{2 j_{2}} \Delta_{j_{2}} \operatorname{Ad}_{\mathcal{P}^{\prime \prime}}\left[B_{p}^{j_{1}, j_{1}^{\prime}}\left(\Pi_{n^{\prime}} \mathcal{U}, t, t^{\prime}\right)\right] \Delta_{j_{2}^{\prime}}=\bar{\Delta}_{j_{2}} \Delta \operatorname{Ad}_{\mathcal{P}^{\prime \prime}}\left[B_{p}^{j_{1}, j_{1}^{\prime}}\left(\Pi_{n^{\prime}} \mathcal{U}, t, t^{\prime}\right)\right] \Delta_{j_{2}^{\prime}} \\
=\bar{\Delta}_{j_{2}} \operatorname{Ad}_{\Delta} \operatorname{Ad}_{\mathcal{P}^{\prime \prime}}\left[B_{p}^{j_{1}, j_{1}^{\prime}}\left(\Pi_{n^{\prime}} \mathcal{U}, t, t^{\prime}\right)\right] \Delta_{j_{2}^{\prime}}+\bar{\Delta}_{j_{2}} \operatorname{Ad}_{\mathcal{P}^{\prime \prime}}\left[B_{p}^{j_{1} j_{1}^{\prime}}\left(\Pi_{n^{\prime}} \mathcal{U}, t, t^{\prime}\right)\right] \bar{\Delta}_{j_{2}^{\prime}} 2^{2 j_{2}^{\prime}} .
\end{aligned}
$$


If we apply estimate (1.2.48) to the right hand side of this equality, we get a bound given by the product of (1.2.48) and of

$$
\left|n^{\prime}\right| 2^{j_{2}}+2^{2 j_{2}^{\prime}} \leq C\left[2^{j_{2}+j_{1}^{\prime}}+2^{2 j_{2}^{\prime}}\right] .
$$

Consequently, we gained the factor

$$
2^{j_{1}^{\prime}-j_{2}}+2^{2\left(j_{2}^{\prime}-j_{2}\right)} \leq C 2^{-\left|j_{2}^{\prime}-j_{2}\right|+\left|j_{1}^{\prime}-j_{2}^{\prime}\right|} .
$$

A symmetric reasoning applies if $j_{2} \leq j_{2}^{\prime}$, so that we have gained, in the special case $N_{1}=1$, the missing factor $2^{N_{1}\left[\left|j_{1}-j_{2}\right|+\left|j_{1}^{\prime}-j_{2}^{\prime}\right|-\left|j_{2}-j_{2}^{\prime}\right|\right]}$. Iterating, we get the case of an arbitrary $N_{1}$.

\section{Symbolic calculus}

The quasi-linear Birkhoff normal forms method, that will be used to prove Theorem 1.2.8, will rely on properties of commutators of operators belonging to the classes defined in Definitions 1.2.3, 1.2.5. To establish such properties, we need a notion of principal symbol that will be defined through local models of our operators, acting on functions defined on the Euclidean space.

\subsection{Operators on $\mathbb{R}^{d}$ and their symbols}

In the same way as we defined dyadic cut-offs acting on functions on the compact manifold $X$ in (1.2.2), we define for $u$ in $\mathcal{S}^{\prime}\left(\mathbb{R}^{d}\right), j$ in $\mathbb{N}$

$$
\begin{aligned}
\Delta_{j}^{\mathrm{E}} u & =\mathcal{F}^{-1}\left(\varphi\left(2^{-j}|\xi|\right) \hat{u}\right), j \geq 1 \\
\Delta_{0}^{\mathrm{E}} u & =S_{0}^{\mathrm{E}} u=\mathcal{F}^{-1}(\psi(|\xi|) \hat{u}) \\
S_{j}^{\mathrm{E}} u & =\sum_{j^{\prime}=0}^{j} \Delta_{j^{\prime}}^{\mathrm{E}} u .
\end{aligned}
$$

where $\hat{u}$ (resp. $\mathcal{F}^{-1}$ ) denotes the Fourier transform (resp. the inverse Fourier transform) on $\mathbb{R}^{d}$, and where $\varphi, \psi$ are the functions introduced at the beginning of subsection 1.2.

Definition 2.1.1 Let $m \in \mathbb{R}, \nu \in \mathbb{N}, p \in \mathbb{N}$. One denotes by $\widetilde{H \Psi}_{p, \nu}^{m, \nu}$ the space of p-linear maps $\mathcal{U}=\left(U_{1}, \ldots, U_{p}\right) \rightarrow A(\mathcal{U})$, defined on $C^{\infty}(X)^{p}$, with values in the space of linear maps from $C_{0}^{\infty}\left(\mathbb{R}^{d}\right)$ to $\mathcal{D}^{\prime}\left(\mathbb{R}^{d}\right)$, such that the distribution kernel of $A(\mathcal{U})$ is supported in a compact subset of $\mathbb{R}^{d} \times \mathbb{R}^{d}$ (independent of $\mathcal{U}$ ) and such that for any family of differential operators $P_{1}, \ldots, P_{k}$ of order $d_{1}, \ldots, d_{k}$, for any $\mathcal{U}$ in $C^{\infty}(X)^{p}$, any $n^{\prime}=\left(n_{1}, \ldots, n_{p}\right)$ in $\left(\mathbb{N}^{*}\right)^{p}$, any $j, j^{\prime}$ in $\mathbb{N}$, any $N_{1}, N_{2}$ in $\mathbb{N}$,

$$
\left\|\Delta_{j}^{\mathrm{E}} \operatorname{Ad}_{P_{1}} \cdots \operatorname{Ad}_{P_{k}} A\left(\Pi_{n^{\prime}} \mathcal{U}\right) \Delta_{j^{\prime}}^{\mathrm{E}}\right\|_{\mathcal{L}\left(L^{2}\right)}
$$

is bounded from above by the right hand side of (1.2.8) 
As in the case of global operators on $X$, one can give an equivalent definition in terms of estimates of the form (1.2.9). We define from $\widehat{H \Psi}_{p, \text { loc }}^{m, \nu}$ the space $H \Psi_{p, \text { loc }}^{m, \nu}$ as in Definition 1.2.3

Definition 2.1.2 Let $m \in \mathbb{R}, \nu \in \mathbb{N}, p \in \mathbb{N}, \sigma>0, R>0$. One defines $\Psi_{p, \text { loc }}^{m, \nu}\left(B_{\sigma}(R)\right)$ as the space of maps $U \rightarrow A(U)$ defined on $B_{\sigma}(R)$, with values in the vector space of linear maps from $C_{0}^{\infty}\left(\mathbb{R}^{d}\right)$ to $\mathcal{D}^{\prime}\left(\mathbb{R}^{d}\right)$, such that the distribution kernel of $A(U)$ is supported in a compact subset of $\mathbb{R}^{d} \times \mathbb{R}^{d}$ independent of $U$, and such that for any family of differential operators $P_{1}, \ldots, P_{k}$ of order $d_{1}, \ldots, d_{k}$, for any $N \in \mathbb{N}$, for any $\sigma^{\prime} \in[-\sigma, \sigma]$, any $U \in B_{\sigma}(R)$, any $H \in H^{\sigma^{\prime}}(X)$, any $j, j^{\prime} \in \mathbb{N},\left\|\Delta_{j}^{\mathrm{E}} \operatorname{Ad}_{P_{1}} \cdots \operatorname{Ad}_{P_{k}} A(U) \Delta_{j^{\prime}}^{\mathrm{E}}\right\|_{\mathcal{L}\left(L^{2}\right)}$ (resp. $\left.\left\|\Delta_{j}^{\mathrm{E}} \operatorname{Ad}_{P_{1}} \cdots \operatorname{Ad}_{P_{k}}\left(\partial_{U} A(U) \cdot H\right) \Delta_{j^{\prime}}^{\mathrm{E}}\right\|_{\mathcal{L}_{\left(L^{2}\right)}}\right)$ is bounded from above by the right hand side of (1.2.16) (resp. (1.2.17)).

As in the case of the global definition, one may give an equivalent characterization in terms of estimates like (1.2.18), (1.2.19).

We introduce classes of symbols that will allow us to give alternative descriptions of the preceding classes of operators.

Definition 2.1.3 Let $m \in \mathbb{R}, \nu \in \mathbb{N}, p \in \mathbb{N}$. One denotes by $\widetilde{H S}_{p, \nu \text { loc }}^{m, \nu}$ the space of functions

$$
\begin{aligned}
a: C^{\infty}(X)^{p} \times T^{*} \mathbb{R}^{d} & \rightarrow \mathbb{C} \\
\left(u_{1}, \ldots, u_{p}, x, \xi\right) & \rightarrow a\left(u_{1}, \ldots, u_{p}, x, \xi\right)
\end{aligned}
$$

which are $p$-linear in $\left(u_{1}, \ldots, u_{p}\right)$, smooth in $(x, \xi)$, and satisfy for any $\alpha, \beta \in \mathbb{N}^{d}$, any $\mathcal{U}=$ $\left(u_{1}, \ldots, u_{p}\right)$ in $C^{\infty}(X)^{p}$, any $n^{\prime}=\left(n_{1}, \ldots, n_{p}\right)$ in $\left(\mathbb{N}^{*}\right)^{p}$, any $(x, \xi) \in T^{*} \mathbb{R}^{d}$, any $N \in \mathbb{N}$

$$
\left|\partial_{x}^{\alpha} \partial_{\xi}^{\beta} a\left(\Pi_{n^{\prime}} \mathcal{U}, x, \xi\right)\right| \leq C_{N}\langle\xi\rangle^{m-|\beta|}\left|n^{\prime}\right|^{\nu(p)+\nu+M_{0}(|\alpha|+|\beta|+d+1)}\left(1+\frac{\left|n^{\prime}\right|}{\langle\xi\rangle}\right)^{-N} \prod_{1}^{p}\left\|\Pi_{n_{\ell}} u_{\ell}\right\|_{L^{2}}
$$

We define also a class of symbols that are not homogeneous in $U$.

Definition 2.1.4 Let $m \in \mathbb{R}, \nu \in \mathbb{N}, p \in \mathbb{N}, \sigma>0, R>0$. One denotes by $S_{p, \text { loc }}^{m, \nu}\left(B_{\sigma}(R)\right)$ the space of maps

$$
\begin{aligned}
a: B_{\sigma}(R) \times T^{*} \mathbb{R}^{d} & \rightarrow \mathbb{C} \\
(U, x, \xi) & \rightarrow a(U, x, \xi)
\end{aligned}
$$

which are smooth in $(x, \xi), C^{1}$ in $U$, and satisfy the following conditions:

(i) For any $\alpha, \beta \in \mathbb{N}^{d}$, there is $C>0$ and, for any $U$ in $B_{\sigma}(R)$, any $(x, \xi)$ in $T^{*} \mathbb{R}^{d}$

$$
\left|\partial_{x}^{\alpha} \partial_{\xi}^{\beta} a(U, x, \xi)\right| \leq C\langle\xi\rangle^{m-|\beta|+\left(M_{0}(|\alpha|+|\beta|+d+1)+\nu(p)+\nu-\sigma\right)_{+}}\|U\|_{H^{\sigma}}^{p} .
$$

(ii) For all $\alpha, \beta, x, \xi, U, H \rightarrow\left(\partial_{x}^{\alpha} \partial_{\xi}^{\beta} \partial_{U} a\right)(U, x, \xi) \cdot H$ extends as a continuous linear form on $H^{\sigma^{\prime}}(X)$ for all $\sigma^{\prime}$ with $\left|\sigma^{\prime}\right| \leq \sigma$ and satisfies

$$
\left|\left(\partial_{x}^{\alpha} \partial_{\xi}^{\beta} \partial_{U} a\right)(U, x, \xi) \cdot H\right| \leq C\langle\xi\rangle^{m-|\beta|+\left(M_{0}(|\alpha|+|\beta|+d+1)+\nu(p)+\nu-\sigma^{\prime}\right)_{+}}\|U\|_{H^{\sigma}}^{p-1}\|H\|_{H^{\sigma^{\prime}}} .
$$


We quantize the preceding classes of symbols in the following way. If $v$ is in $C_{0}^{\infty}\left(\mathbb{R}^{d}\right)$ and $a$ is in $S_{p, \text { loc }}^{m, \nu}\left(B_{\sigma}(R)\right)$, we set

$$
\mathrm{Op}(a(U ; \cdot)) v=\frac{1}{(2 \pi)^{d}} \int_{\mathbb{R}^{d}} e^{i x \cdot \xi} a(U, x, \xi) \hat{v}(\xi) d \xi .
$$

We use a similar formula to quantize elements of $\widetilde{H S}_{p, \nu}^{m, \nu}$.

If $\chi$ is in $C_{0}^{\infty}\left(\mathbb{R}^{d}\right), a$ belongs to $\widetilde{H S}_{p, \nu}^{m, \nu}$, we set

$$
a_{\chi}(\mathcal{U}, x, \xi)=\chi\left(D_{x} /\langle\xi\rangle\right)[a(\mathcal{U}, x, \xi)]=\mathcal{F}_{\eta}^{-1}[\chi(\eta /\langle\xi\rangle) \hat{a}(\mathcal{U}, \eta, \xi)]
$$

where $\hat{a}(\mathcal{U}, \eta, \xi)$ is the $x$-Fourier transform of $a(\mathcal{U}, x, \xi)$. We use a similar notation for elements of $S_{p, l o c}^{m, \nu}\left(B_{\sigma}(R)\right)$. The definition immediately implies that $a_{\chi}$ belongs to the same space of symbols as $a$.

If $\chi$ is supported in a small enough neighborhood of zero, on the support of $\widehat{a_{\chi}}(\mathcal{U}, \eta, \xi),|\eta| \ll|\xi|$, so that $\langle\xi+\eta\rangle \sim\langle\xi\rangle$. For technical reasons, we shall have to consider symbols for which a weaker version of this spectral localization is satisfied, namely symbols a in $\widetilde{H S}_{p, \nu}^{m, \nu}$ or $S_{p, \text { loc }}^{m, \nu}\left(B_{\sigma}(R)\right)$ such that for any $\lambda \in \mathbb{R},\left\langle D_{x}+\xi\right\rangle^{\lambda}\langle\xi\rangle^{-\lambda} a$ is still in the same class. We shall use eventually the following lemma:

Lemma 2.1.5 Let a be an element of $\widetilde{H S}_{p, \text { loc }}^{m, \nu}\left(\right.$ resp. $\left.S_{p, \text { loc }}^{m, \nu}\left(B_{\sigma}(R)\right)\right)$ such that $\left\langle D_{x}+\xi\right\rangle^{\lambda}\langle\xi\rangle^{-\lambda} a$ is in the same space for any $\lambda \in \mathbb{R}$. Let $\theta$ in $\mathcal{S}\left(\mathbb{R}^{d} \times \mathbb{R}^{d}\right)$ and $b(\mathcal{U}, x, y, \xi)=\theta(x, y) a(\mathcal{U}, x, \xi)$ (resp. $b(U, x, y, \xi)=\theta(x, y) a(U, x, \xi))$. Then, for any $n^{\prime}$ in $\left(\mathbb{N}^{*}\right)^{p}$, any $\lambda$ in $\mathbb{R}$,

$$
\left|\partial_{x}^{\alpha} \partial_{\xi}^{\beta} \partial_{y}^{\gamma}\left[\left\langle D_{x}+\xi\right\rangle^{\lambda}\langle\xi\rangle^{-\lambda} b\left(\Pi_{n^{\prime}} \mathcal{U}, x, y, \xi\right)\right]\right|,
$$

resp.

$$
\left|\partial_{x}^{\alpha} \partial_{\xi}^{\beta} \partial_{y}^{\gamma}\left[\left\langle D_{x}+\xi\right\rangle^{\lambda}\langle\xi\rangle^{-\lambda} b(U, x, y, \xi)\right]\right|
$$

resp.

$$
\left|\partial_{x}^{\alpha} \partial_{\xi}^{\beta} \partial_{y}^{\gamma}\left[\left\langle D_{x}+\xi\right\rangle^{\lambda}\langle\xi\rangle^{-\lambda}\left(\partial_{U} b(U, x, y, \xi) \cdot H\right)\right]\right|
$$

is bounded from above by the right hand side of (2.1.2) (resp. (2.1.3), resp. (2.1.4)).

Proof: It is enough to prove that the operator $\left\langle D_{x}+\xi\right\rangle^{\lambda} \theta\left\langle D_{x}+\xi\right\rangle^{-\lambda}-\theta$ is bounded on $L^{\infty}(d x)$, uniformly in $\xi, y$, for any $\lambda \in \mathbb{R}$. The distribution kernel of this operator, at fixed $\xi, y$, is

$$
K_{\xi, y}\left(x, x^{\prime}\right)=\frac{1}{(2 \pi)^{2 d}} e^{-i\left(x-x^{\prime}\right) \cdot \xi} \int e^{i\left(x-x^{\prime}\right) \cdot \eta+i x \cdot \zeta}\langle\eta\rangle^{-\lambda}\left[\langle\eta+\zeta\rangle^{\lambda}-\langle\eta\rangle^{\lambda}\right] \hat{\theta}(\zeta, y) d \zeta d \eta,
$$

where $\hat{\theta}(\zeta, y)$ is the Fourier transform relatively to the first variable. Integrating by parts, one checks

$$
\left|K_{\xi, y}\left(x, x^{\prime}\right)\right| \leq C_{N}\left|x-x^{\prime}\right|^{-d+1}\left\langle x-x^{\prime}\right\rangle^{-N}\langle x\rangle^{-N}
$$

for any $N$, from which the $\mathcal{L}\left(L^{\infty}\right)$-bound follows.

We relate the symbols we just defined to the local operators. 
Proposition 2.1.6 Let $m \in \mathbb{R}, \nu \in \mathbb{N}, \sigma>0, R>0$.

(i) Let $A$ be an element of $\widetilde{H \Psi}_{p, \text { loc }}^{m, \nu}$ (resp. $\left.\Psi_{p, \text { loc }}^{m, \nu}\left(B_{\sigma}(R)\right)\right)$. There is a (unique) symbol a in $\widetilde{H S} S_{p, \text { loc }}^{m, \nu}$ (resp. $S_{p, \text { loc }}^{m, \nu}\left(B_{\sigma}(R)\right)$ ) such that for any $\lambda \in \mathbb{R},\left\langle D_{x}+\xi\right\rangle^{\lambda}\langle\xi\rangle^{-\lambda} a$ is still in the same space, and such that $A(\mathcal{U})=\operatorname{Op}(a(\mathcal{U} ; \cdot))($ resp. $A(U)=\operatorname{Op}(a(U ; \cdot)))$.

(ii) Let a be a symbol in $\widetilde{H S} S_{p, \text { loc }}^{m, \nu}$ (resp. $\left.S_{p, \text { loc }}^{m, \nu}\left(B_{\sigma}(R)\right)\right)$ such that for any $\lambda \in \mathbb{R},\left\langle D_{x}+\xi\right\rangle^{\lambda}\langle\xi\rangle^{-\lambda} a$ is still in the same space. Let $\theta_{1}, \theta_{2}$ in $C_{0}^{\infty}\left(\mathbb{R}^{d}\right)$. Then $\theta_{1} \mathrm{Op}(a) \theta_{2}$ belongs to $\widetilde{H \Psi}_{p, \text { loc }}^{m, \nu+2 M_{0}(d+1)}$ (resp. $\Psi_{p, \text { loc }}^{m, 2+2 M_{0}(d+1)}\left(B_{\sigma}(R)\right)$ ).

(iii) Let a be a symbol in $\widetilde{H S}_{p, \text { loc }}^{m, \nu}$ (resp. $S_{p, \text { loc }}^{m, \nu}\left(B_{\sigma}(R)\right)$ ). Let $\chi$ be in $C_{0}^{\infty}\left(\mathbb{R}^{d}\right)$ with small enough support, $\chi \equiv 1$ close to zero. Then, for any $j \geq 0, a-a_{\chi}$ is in $\widetilde{H S}_{p, \text { loc }}^{m-j+j M_{0}}$ (resp. $\left.S_{p, \text { loc }}^{m-j+j M_{0}}\left(B_{\sigma}(R)\right)\right)$.

Proof: (i) Let $A$ be an element of $\widetilde{H \Psi}_{p, \nu}^{m, \nu}$. Denote by $K(\mathcal{U}, x, y)$ the distribution kernel of $A(\mathcal{U})$ and define

$$
a(\mathcal{U}, x, \xi)=\int_{\mathbb{R}^{d}} K(\mathcal{U}, x, y) e^{-i(x-y) \cdot \xi} d y
$$

so that $\operatorname{Op}(a(\mathcal{U} ; \cdot))=A(\mathcal{U})$ and $\operatorname{Op}\left(\partial_{x}^{\alpha}\left(i \partial_{\xi}\right)^{\beta} a(\mathcal{U} ; \cdot)\right)=\left(\operatorname{Ad}_{\partial_{x}}\right)^{\alpha}\left(\operatorname{Ad}_{x}\right)^{\beta} A(\mathcal{U})$. Since $K$ is compactly supported, we may choose $\theta_{1}, \theta_{2}$ in $C_{0}^{\infty}\left(\mathbb{R}^{d}\right)$ such that $K=\theta_{1}(x) K \theta_{2}(y)$. We compute

$$
\begin{aligned}
\hat{a}(\mathcal{U}, \eta, \xi) & =\int e^{-i x \cdot(\xi+\eta)} \theta_{1}(x) K(\mathcal{U}, x, y) e^{i y \cdot \xi} \theta_{2}(y) d y d \xi d x \\
& =\int e^{-i x \cdot(\xi+\eta)} \theta_{1}(x) A(\mathcal{U})\left[e^{i\left\langle\xi \cdot{ }^{\prime}\right.} \theta_{2}(\cdot)\right] d x .
\end{aligned}
$$

This implies, for any $\lambda \in \mathbb{R}$,

$$
\left|\widehat{\partial_{x}^{\alpha} \partial_{\xi}^{\beta}} a\left(\Pi_{n^{\prime}} \mathcal{U}, \eta, \xi\right)\right| \leq\left\|e^{-i\langle\cdot, \xi+\eta\rangle} \theta_{1}\right\|_{H^{-\lambda}}\left\|\left(\operatorname{Ad}_{\partial_{x}}\right)^{\alpha}\left(\operatorname{Ad}_{x}\right)^{\beta} A\left(\Pi_{n^{\prime}} \mathcal{U}\right)\left[e^{i\langle\xi, \cdot\rangle} \theta_{2}\right]\right\|_{H^{\lambda}} .
$$

Using that $\left\|e^{-i\left\langle\zeta \cdot{ }^{\prime}\right.} \theta_{j}\right\|_{H^{\lambda}} \sim\langle\zeta\rangle^{\lambda}$ when $|\zeta| \rightarrow+\infty$ and inequality (1.2.9), we obtain

$$
\left.\widehat{\partial_{x}^{\alpha} \partial_{\xi}^{\beta}} a\left(\Pi_{n^{\prime}} \mathcal{U}, \eta, \xi\right)|| n^{\prime}\right|^{N_{2}} \leq C\langle\xi+\eta\rangle^{-\lambda}\langle\xi\rangle^{\lambda+N_{2}+m-|\beta|}\left|n^{\prime}\right|^{\nu(p)+\nu+M_{0}(|\alpha|+|\beta|)} \prod_{1}^{p}\left\|\Pi_{n_{\ell}} u_{\ell}\right\|_{L^{2}} .
$$

By Sobolev injection, we get the same estimate for

$$
\left|\partial_{x}^{\alpha} \partial_{\xi}^{\beta}\left(\left\langle D_{x}+\xi\right\rangle^{\lambda}\langle\xi\rangle^{-\lambda} a\left(\Pi_{n^{\prime}} \mathcal{U}, x, \xi\right)\right)\right|
$$

if we replace in the right hand side $\nu$ by $\nu+M_{0}(d+1)$ and discard $\langle\xi+\eta\rangle^{-\lambda}\langle\xi\rangle^{\lambda}$. This gives the wanted estimate (2.1.2), showing that for any $\lambda,\left\langle D_{x}+\xi\right\rangle^{\lambda}\langle\xi\rangle^{-\lambda} a$ is in $\widetilde{H S}_{p, \nu \text { loc }}^{m,}$.

The proof of the analogous statement for operators in $\Psi_{p, \text { loc }}^{m, \nu}\left(B_{\sigma}(R)\right)$ is similar, using (1.2.18) and (1.2.19). 
(ii) Let $a$ be in $\widetilde{H S}_{p, \text { loc }}^{m, \nu}$. Since $\theta_{1} \mathrm{Op}(a) \theta_{2}$,satisfies the support property, we just need to check estimates (1.2.8) for the operator $\operatorname{Ad}_{P_{1}} \cdots \operatorname{Ad}_{P_{k}}\left[\theta_{1} \mathrm{Op}\left(a\left(\Pi_{n^{\prime}} \mathcal{U} ; \cdot\right)\right) \theta_{2}\right]$. By the last remark following Definition 1.2.2, we may assume that $P_{\ell}$ is of order zero or one, so either of the form $b(x)$ or $b(x) \frac{\partial}{\partial x_{j}}$ for some function $b$ in $C_{0}^{\infty}\left(\mathbb{R}^{d}\right)$. Let $\alpha$ (resp. $\beta$ ) be the number of those $\ell$ such that $P_{\ell}$ is of order 1 (resp. 0). The kernel of $\left[b(x) \frac{\partial}{\partial x_{j}}, \theta_{1} \mathrm{Op}(a(\mathcal{U} ; \cdot)) \theta_{2}\right]\left(\operatorname{resp} .\left[b(x), \theta_{1} \mathrm{Op}(a(\mathcal{U} ; \cdot)) \theta_{2}\right]\right)$ is

$$
\frac{1}{(2 \pi)^{d}} \int e^{i(x-y) \cdot \xi} \tilde{a}(\mathcal{U}, x, y, \xi) d \xi
$$

with

$$
\begin{aligned}
\tilde{a}(\mathcal{U}, x, y, \xi)=-\sum_{k=1}^{d} b_{k}(x, y) \theta_{1}(x) \theta_{2}(y) \frac{\partial}{\partial \xi_{k}}\left[a(\mathcal{U}, x, \xi) \xi_{j}\right] & +b(x) \frac{\partial}{\partial x_{j}}\left[\theta_{1}(x) a(\mathcal{U}, x, \xi) \theta_{2}(y)\right] \\
& +\frac{\partial}{\partial y_{j}}\left[b(y) \theta_{1}(x) a(\mathcal{U}, x, \xi) \theta_{2}(y)\right]
\end{aligned}
$$

with $b_{k}$ a smooth function on $\mathbb{R}^{d} \times \mathbb{R}^{d}$ (resp. with

$$
\left.\tilde{a}(\mathcal{U}, x, y, \xi)=i \sum_{k=1}^{d} b_{k}(x, y) \theta_{1}(x) \frac{\partial a}{\partial \xi_{k}}(\mathcal{U}, x, \xi) \theta_{2}(y)\right) .
$$

Computing in the same way iterated commutators, and using Lemma 2.1.5, we conclude that $\operatorname{Ad}_{P_{1}} \cdots \operatorname{Ad}_{P_{k}}\left[\theta_{1} \operatorname{Op}(a(\mathcal{U} ; \cdot)) \theta_{2}\right]=\operatorname{Op}(b(\mathcal{U} ; \cdot))$, where, for any $\lambda \in \mathbb{R}$, any $N \in \mathbb{N}$,

$$
\begin{aligned}
\left|\partial_{x}^{\alpha^{\prime}} \partial_{\xi}^{\beta^{\prime}} \partial_{y}^{\gamma^{\prime}}\left[\left\langle D_{x}+\xi\right\rangle^{\lambda}\langle\xi\rangle^{-\lambda} b\left(\Pi_{n^{\prime}} \mathcal{U}, x, y, \xi\right)\right]\right| \leq C\langle\xi\rangle^{m-\beta-\left|\beta^{\prime}\right|}\left|n^{\prime}\right|^{\nu(p)+\nu+M_{0}\left(\alpha+\beta+\left|\alpha^{\prime}\right|+\left|\beta^{\prime}\right|+d+1\right)} & \times\left(1+\frac{\left|n^{\prime}\right|}{\langle\xi\rangle}\right)^{-N} \prod_{1}^{p}\left\|\Pi_{n_{\ell}} u_{\ell}\right\|_{L^{2}}
\end{aligned}
$$

and where $\operatorname{Op}(b)$ is the operator with kernel $(2 \pi)^{-d} \int e^{i(x-y) \cdot \xi} b(\mathcal{U}, x, y, \xi) d \xi$. Let us estimate $\left\|\Delta_{j} \operatorname{Op}\left(b\left(\Pi_{n^{\prime}} \mathcal{U} ; \cdot\right)\right) \Delta_{j^{\prime}}\right\|_{\mathcal{L}\left(L^{2}\right)}$. We decompose $b(\mathcal{U}, x, y, \xi)=\sum_{j^{\prime \prime}} b_{j^{\prime \prime}}(\mathcal{U}, x, y, \xi)$ with the notation $b_{j^{\prime \prime}}=b \varphi\left(2^{-j^{\prime \prime}}|\xi|\right)$ for $j \geq 1, b_{0}=b \psi(|\xi|)$. The kernel $K_{j^{\prime \prime}}(\mathcal{U}, x, y)$ of $\Delta_{j} \operatorname{Op}\left(b_{j^{\prime \prime}}\right) \Delta_{j^{\prime}}$ may be written by a direct computation

$$
K_{j^{\prime \prime}}(\mathcal{U}, x, y)=\frac{1}{(2 \pi)^{d}} \int e^{i(x-y) \cdot \xi} c_{j^{\prime \prime}}(\mathcal{U}, x, y, \xi) d \xi
$$

with

$$
\begin{aligned}
c_{j^{\prime \prime}}(\mathcal{U}, x, y, \xi)=\frac{1}{(2 \pi)^{2 d}} \int e^{i\left[z \zeta+z^{\prime} \zeta^{\prime}\right]} \varphi\left(2^{-j}|\xi-\zeta|\right) & \varphi\left(2^{-j^{\prime}}\left|\xi-\zeta^{\prime}\right|\right) \varphi\left(2^{-j^{\prime \prime}}|\xi|\right) \\
& \times b\left(\mathcal{U}, x-z, y-z^{\prime}, \xi\right) d z d z^{\prime} d \zeta d \zeta^{\prime}
\end{aligned}
$$

when $j^{\prime \prime}>0, j>0, j^{\prime}>0$, and similar expressions when $j, j^{\prime}$ or $j^{\prime \prime}$ is zero. If we write

$$
b\left(\mathcal{U}, x-z, y-z^{\prime}, \xi\right)=\left\langle D_{z}-\xi\right\rangle^{-\lambda}\left\langle D_{x}+\xi\right\rangle^{\lambda} b\left(\mathcal{U}, x-z, y-z^{\prime}, \xi\right)
$$


and integrate by parts in $z$, we get the expression

$$
\begin{aligned}
c_{j^{\prime \prime}}\left(\Pi_{n^{\prime}} \mathcal{U}, x, y, \xi\right)=\frac{1}{(2 \pi)^{2 d}}\langle\xi\rangle^{\lambda} 2^{-j \lambda} \int e^{i\left[z \zeta+z^{\prime} \zeta^{\prime}\right]} \phi_{j}\left(2^{-j}|\xi-\zeta|\right) \varphi\left(2^{-j^{\prime}}\left|\xi-\zeta^{\prime}\right|\right) \varphi\left(2^{-j^{\prime \prime}}|\xi|\right) \\
\times \tilde{b}\left(\mathcal{U}, x-z, y-z^{\prime}, \xi\right) d z d z^{\prime} d \zeta d \zeta^{\prime}
\end{aligned}
$$

where $\tilde{b}$ is the symbol in the left hand side of (2.1.7), and where $\phi_{j}$ has the same support properties as $\varphi$, with estimates of its derivatives uniform in $j$. Let us show that, for any $N, N^{\prime}$

$$
\begin{aligned}
\left|\partial_{\xi}^{\beta^{\prime}} c_{j^{\prime \prime}}\left(\Pi_{n^{\prime}} \mathcal{U}, x, y, \xi\right)\right| \leq C 2^{j^{\prime \prime}(m-\beta)}\left[\max \left(2^{-j}, 2^{-j^{\prime}}, 2^{-j^{\prime \prime}}\right)\right]^{\left|\beta^{\prime}\right|} 2^{-N^{\prime}\left(\left|j-j^{\prime}\right|+\left|j^{\prime}-j^{\prime \prime}\right|\right)} & \\
& \times\left|n^{\prime}\right|^{\nu(p)+\nu+M_{0}\left(\alpha+\beta+d+1+\left|\beta^{\prime}\right|\right)}\left(1+2^{-j^{\prime \prime}}\left|n^{\prime}\right|\right)^{-N} \prod_{1}^{p}\left\|u_{\ell}\right\|_{L^{2}}
\end{aligned}
$$

To do so, we perform $N_{1}$ integrations by parts in (2.1.8) using the vector field $L=\left(1+\left|\zeta^{\prime}\right|^{2}\right)^{-1}(1+$ $\left.\zeta^{\prime} \cdot D_{z^{\prime}}\right)$. The estimate (2.1.7) show that we gain in that way a $\left\langle\zeta^{\prime}\right\rangle^{-N_{1}}$ factor. Next, perform $N_{2}$ integrations by parts using $L_{2}=\left(1+\left|z^{\prime}\right|^{2}\right)^{-1}\left(1+z^{\prime} \cdot D_{\zeta^{\prime}}\right)$. We gain a factor $\left\langle z^{\prime}\right\rangle^{-N_{2}}$. Finally, we integrate by parts using $L_{3}=\left(1+2^{2 j}|z|^{2}\right)^{-1}\left(1+2^{2 j} z \cdot D_{\zeta}\right)$ to gain a factor $\left\langle 2^{j} z\right\rangle^{-N_{3}}$. We obtain for the modulus of $\partial_{\xi}^{\beta^{\prime}} c_{j^{\prime \prime}}$ a bound given by the product of

$$
C 2^{j^{\prime \prime}(m-\beta)}\left[\max \left(\left(2^{-j}, 2^{-j^{\prime}}, 2^{-j^{\prime \prime}}\right)\right]^{\left|\beta^{\prime}\right|}\left|n^{\prime}\right|^{\nu(p)+\nu+M_{0}\left(\alpha+\beta+d+1+\left|\beta^{\prime}\right|\right)}\left(1+2^{-j^{\prime \prime}}\left|n^{\prime}\right|\right)^{-N} \prod_{1}^{p}\left\|u_{\ell}\right\|_{L^{2}}\right.
$$

and of

$$
2^{\left(j^{\prime \prime}-j\right) \lambda} \int \mathbb{1}_{|\xi-\zeta| \sim 2^{j}} \mathbb{1}_{\left|\xi-\zeta^{\prime}\right| \sim 2^{j^{\prime}}} \mathbb{1}_{|\xi| \sim 2^{j^{\prime \prime}}}\left\langle\zeta^{\prime}\right\rangle^{-N_{1}}\left\langle z^{\prime}\right\rangle^{-N_{2}}\left\langle 2^{j} z\right\rangle^{-N_{3}} d z d z^{\prime} d \zeta d \zeta^{\prime} .
$$

If we integrate for $\left|\zeta^{\prime}\right| \geq c 2^{\left|j^{\prime}-j^{\prime \prime}\right|}$ for some $c>0$, we get, choosing $\lambda$ conveniently and $N_{1}$ large enough, a factor $O\left(2^{-N^{\prime}\left[\left|j-j^{\prime}\right|+\left|j^{\prime}-j^{\prime \prime}\right|\right]}\right)$, whence the upper bound (2.1.9). If we integrate for $\left|\zeta^{\prime}\right| \ll 2^{\left|j^{\prime}-j^{\prime \prime}\right|}$, the cut-offs show that $\left|j^{\prime}-j^{\prime \prime}\right|$ has to stay smaller than a fixed constant, so that we get also the wanted estimate (2.1.9). If we go back to the kernel $K_{j^{\prime \prime}}$, perform $d+1$ integrations by parts in $\xi$ and use (2.1.9), we get a bound

$$
\begin{aligned}
\left|K_{j^{\prime \prime}}\left(\Pi_{n^{\prime}} \mathcal{U}, x, y\right)\right| \leq C 2^{j^{\prime \prime}} d 1 & \left.+\min \left(2^{j}, 2^{j^{\prime}}, 2^{j^{\prime \prime}}\right)|x-y|\right]^{-d-1} 2^{-N^{\prime}\left[\left|j-j^{\prime}\right|+\left|j^{\prime}-j^{\prime \prime}\right|\right]} \\
& \times 2^{j^{\prime \prime}(m-\beta)}\left|n^{\prime}\right|^{\nu(p)+\nu+M_{0}(\alpha+\beta+2(d+1))}\left(1+2^{-j^{\prime \prime}}\left|n^{\prime}\right|\right)^{-N} \prod_{1}^{p}\left\|u_{\ell}\right\|_{L^{2}} .
\end{aligned}
$$

The $\mathcal{L}\left(L^{2}\right)$-norm of $\Delta_{j} \mathrm{Op}(b) \Delta_{j^{\prime}}$ is bounded from above by the sum in $j^{\prime \prime}$ of the $L^{1}(d x)$ (or $\left.L^{1}(d y)\right)$ norm of $K_{j^{\prime \prime}}\left(\Pi_{n^{\prime}} \mathcal{U}, x, y\right)$, so by

$$
C 2^{j(m-\beta)}\left|n^{\prime}\right|^{\nu(p)+\nu+M_{0}(\alpha+\beta+2(d+1))}\left(1+2^{-j}\left|n^{\prime}\right|\right)^{-N} 2^{-N^{\prime}\left|j-j^{\prime}\right|} \prod_{1}^{p}\left\|u_{\ell}\right\|_{L^{2}}
$$

for some new value of $N^{\prime}$. This shows that $\theta_{1} \mathrm{Op}(a) \theta_{2}$ is in $\widetilde{H \Psi}_{p, \nu \text { loc }}^{m+2 M_{0}(d+1)}$ as claimed.

The proof of the corresponding statement in the framework of the $S_{p, \text { loc }}^{m, \nu}\left(B_{\sigma}(R)\right)$ class of symbols is identical. 
(iii) This statement follows from the formula

$$
\left(a-a_{\chi}\right)(\mathcal{U}, x, \xi)=\left[\left(\frac{D_{x}}{\langle\xi\rangle}\right)^{-j}(1-\chi)\left(\frac{D_{x}}{\langle\xi\rangle}\right)\right]\left(\frac{D_{x}}{\langle\xi\rangle}\right)^{j} a
$$

for any $j>0$.

We have already obtained, in the global framework, results of composition of operators (see Lemma 1.2.4 and Lemma 1.2.6). Nevertheless, to see that a commutator between two operators is one order less than the sum of the orders, we need to study, for local operators, the symbol of the composition.

Theorem 2.1.7 Let $m, m^{\prime}$ be real numbers, $\nu, \nu^{\prime}$ in $\mathbb{R}_{+}, \sigma>\min (\nu(p), \nu(q))+\min \left(\nu, \nu^{\prime}\right)+$ $M_{0}(2 d+3)$. Assume that

$$
\nu(p)+\nu(q)+\nu^{\prime}+M_{0}(2 d+3) \leq \nu(p+q), p>0, q>0 .
$$

Let $A$ be in $\widetilde{H \Psi} \Psi_{p, \text { loc }}^{m, \nu}\left(\right.$ resp. $\left.\Psi_{p, \text { loc }}^{m, \nu}\left(B_{\sigma}(R)\right)\right)$ and $B$ be in $\widetilde{H \Psi} \Psi_{q, \text { loc }}^{m^{\prime}, \nu^{\prime}}\left(\right.$ resp. $\left.\Psi_{q, \text { loc }}^{m^{\prime}, \nu^{\prime}}\left(B_{\sigma}(R)\right)\right)$. Denote by $a$ and $b$ the element of $\widetilde{H S} S_{p, \nu \text { loc }}^{m, \nu}\left(\right.$ resp. $\left.S_{p, \text { loc }}^{m, \nu}\left(B_{\sigma}(R)\right)\right)$ and $\widetilde{H S}{ }_{q, \text { loc }}^{m^{\prime}, \nu^{\prime}}\left(\right.$ resp. $\left.S_{q, \mathrm{loc}}^{m^{\prime}, \nu^{\prime}}\left(B_{\sigma}(R)\right)\right)$ given by (i) of Proposition 2.1.6. Let $\chi \in C_{0}^{\infty}\left(\mathbb{R}^{d}\right)$ be equal to one close to zero, with small enough support. Then, if $\theta \in C_{0}^{\infty}\left(\mathbb{R}^{d}\right)$ is equal to one on a large enough compact subset of $\mathbb{R}^{d}$, we may write for any $\mathcal{U}^{\prime}$ in $C^{\infty}(X)^{p}, \mathcal{U}^{\prime \prime}$ in $C^{\infty}(X)^{q}$

$$
A\left(\mathcal{U}^{\prime}\right) \circ B\left(\mathcal{U}^{\prime \prime}\right)=\theta \operatorname{Op}\left[\left(a\left(\mathcal{U}^{\prime}, \cdot\right) b\left(\mathcal{U}^{\prime \prime}, \cdot\right)\right)_{\chi}\right] \theta+C\left(\mathcal{U}^{\prime}, \mathcal{U}^{\prime \prime}\right),
$$

resp. for $U$ in $B_{\sigma}(R)$,

$$
A(U) \circ B(U)=\theta \operatorname{Op}\left[(a(U, \cdot) b(U, \cdot))_{\chi}\right] \theta+C(U),
$$

where $C$ is an element of $\widetilde{H \Psi}_{p+q, \text { loc }}^{m+m^{\prime}-1, \nu+M_{0}(2 d+3)}$ (resp. $\left.\Psi_{\max (p, q), \operatorname{loc}}^{m+m^{\prime}-1, \max \left(\nu, \nu^{\prime}\right)+M_{0}(2 d+3)}\left(B_{\sigma}(R)\right)\right)$ if $p>0, q>0$ and of $\widetilde{H \Psi}_{p+q, \text { loc }}^{m+m^{\prime}-1, \nu+\nu^{\prime}+2 M_{0}(2 d+3)}$ (resp. $\left.\Psi_{\max (p, q), \operatorname{loc}}^{m+m^{\prime}-1, \max \left(\nu, \nu^{\prime}\right)+M_{0}(2 d+3)}\left(B_{\sigma}(R)\right)\right)$ if $p=0$ or $q=0$.

Remark: Of course, if one assumes instead of (2.1.10), $\nu(p)+\nu(q)+\nu+M_{0}(2 d+3) \leq \nu(p+q)$ for $p>0, q>0$, one gets the same conclusion with $\nu$ and $\nu^{\prime}$ exchanged.

Proof: Let $A$ be in $\widetilde{H \Psi}_{p, \nu}^{m, \nu}$ and $B$ in $\widetilde{H \Psi}_{q \text {, loc }}^{m^{\prime}, \nu^{\prime}}$. Let $\theta, \theta_{1}$ be in $C_{0}^{\infty}\left(\mathbb{R}^{d}\right)$, with $\theta_{1} \theta=\theta$ and $\theta A \theta=A, \theta B \theta=B$. By (iii) of Proposition 2.1.6, for any $\lambda \in \mathbb{R},\left\langle D_{x}+\xi\right\rangle^{\lambda}\langle\xi\rangle^{-\lambda}\left(a-a_{\chi}\right)$ is in $\widetilde{H S}_{p \text {,loc }}^{m-1, \nu+M_{0}}$ and we have a similar statement with $b$. Applying (ii) of Proposition 2.1.6, we may write

$$
A=\theta_{1} \mathrm{Op}\left(a_{\chi}\right) \theta_{1}+A_{1}, B=\theta_{1} \mathrm{Op}\left(b_{\chi}\right) \theta_{1}+B_{1}
$$

with $A_{1} \in \widetilde{H \Psi}_{p, \text { loc }}^{m-1, \nu+M_{0}(2 d+3)}$ and $B_{1} \in \widetilde{H \Psi}_{q, \text { loc }}^{m^{\prime}-1, \nu^{\prime}+M_{0}(2 d+3)}$. Using (2.1.10) and Lemma 1.2.4, we write

$$
A B=\theta_{1} \mathrm{Op}\left(a_{\chi}\right) \theta_{1}^{2} \mathrm{Op}\left(b_{\chi}\right) \theta_{1}+C_{1}
$$


where $C_{1}$ is a contribution to $C$ in (2.1.11). The first term in the right hand side may be written $\theta_{1} \mathrm{Op}(c(\mathcal{U}, \cdot)) \theta_{1}$ where

$$
c(\mathcal{U}, x, \xi)=\frac{1}{(2 \pi)^{d}} \int e^{-i z \cdot \zeta} a_{\chi}\left(\mathcal{U}^{\prime}, x, \xi-\zeta\right) \theta_{1}^{2}(x-z) b_{\chi}\left(\mathcal{U}^{\prime \prime}, x-z, \xi\right) d z d \zeta
$$

if $\mathcal{U} \in C^{\infty}(X)^{p+q}$ has been split in $\mathcal{U}=\left(\mathcal{U}^{\prime}, \mathcal{U}^{\prime \prime}\right) \in C^{\infty}(X)^{p} \times C^{\infty}(X)^{q}$. Then

$$
\begin{array}{r}
c(\mathcal{U}, x, \xi)-a_{\chi} b_{\chi}(\mathcal{U}, x, \xi) \theta_{1}(x)^{2}=\frac{1}{(2 \pi)^{d}} \int e^{-i z \cdot \zeta}\left[a_{\chi}\left(\mathcal{U}^{\prime}, x, \xi-\zeta\right)-a_{\chi}\left(\mathcal{U}^{\prime}, x, \xi\right)\right] \theta_{1}^{2}(x-z) \\
\times b_{\chi}\left(\mathcal{U}^{\prime \prime}, x-z, \xi\right) d z d \zeta .
\end{array}
$$

The right hand side may be written, performing an integration by parts, as

$$
\frac{1}{(2 \pi)^{d}} \int_{0}^{1} \int e^{-i z \cdot \zeta}\left(\partial_{\xi} a_{\chi}\right)\left(\mathcal{U}^{\prime}, x, \xi-t \zeta\right) D_{x}\left[\theta_{1}^{2}(x-z) b_{\chi}\left(\mathcal{U}^{\prime \prime}, x-z, \xi\right)\right] d z d \zeta d t
$$

We perform $d+1$ integrations by parts using the vector field $L=\left(1+\zeta^{2}\right)^{-1}\left(1-\zeta \cdot D_{z}\right)$ to get an absolutely convergent integral in $\zeta$. If we replace in $(2.1 .15) \mathcal{U}^{\prime}$ by $\Pi_{n^{\prime}} \mathcal{U}^{\prime}, \mathcal{U}^{\prime \prime}$ by $\Pi_{n^{\prime \prime}} \mathcal{U}^{\prime \prime}$, each $D_{z}$ derivative acting on $b_{\chi}$ makes lose a power $\left|n^{\prime \prime}\right|^{M_{0}}$. We decompose $\theta_{1}^{2}$ as

$$
\theta_{1}^{2}(w)=\left(\chi(D /\langle\xi\rangle) \theta_{1}^{2}\right)(w)+\tilde{\theta}(w, \xi)
$$

where $\left|\partial_{w}^{\gamma} \tilde{\theta}(w, \xi)\right|=O\left(\langle\xi\rangle^{-N}\langle w\rangle^{-N}\right)$ for any $N, \gamma$. Let us study first the contribution to (2.1.15) coming from the first term in the right hand side of (2.1.16). If Supp $\chi$ is small enough, the support properties of $\widehat{b_{\chi}}\left(\mathcal{U}^{\prime \prime}, \eta, \xi\right)$ imply that, in (2.1.15), we may assume $|\zeta| \ll|\xi|$ on the domain of integration. This integral, computed at $\Pi_{n} \mathcal{U}$ instead of $\mathcal{U}$, is thus bounded from above in modulus by

$$
C\left|n^{\prime}\right|^{\nu(p)+\nu+M_{0}(d+2)}\left|n^{\prime \prime}\right|^{\nu(q)+\nu^{\prime}+M_{0}(2 d+3)}\langle\xi\rangle^{m+m^{\prime}-1}\left(1+\frac{\left|n^{\prime}\right|}{\langle\xi\rangle}\right)^{-N}\left(1+\frac{\left|n^{\prime \prime}\right|}{\langle\xi\rangle}\right)^{-N} \prod_{1}^{p+q}\left\|\Pi_{n_{\ell}} u_{\ell}\right\|_{L^{2}} .
$$

Using (2.1.10), we get estimates (2.1.2) of an element of $\widetilde{H S}_{p+q, \text { loc }}^{m+m^{\prime}-1, \nu+M_{0}}$ if $p, q>0$ and of $\widetilde{H S}_{p+q, \text { loc }}^{m+m^{\prime}-1, \nu+\nu^{\prime}+M_{0}(2 d+4)}$ if $p$ or $q$ is zero. The derivatives are bounded in the same way, and the symbol obtained making act $\left\langle D_{x}+\xi\right\rangle^{\lambda}\langle\xi\rangle^{-\lambda}$ on (2.1.15) satisfies similar estimates, again because the $x$-Fourier transform of (2.1.15) is supported for $|\eta| \ll|\xi|$ if $\operatorname{Supp} \chi$ is small enough. If we consider the contribution of $\tilde{\theta}$ in (2.1.16) to (2.1.15), and use that $\partial_{w}^{\gamma} \tilde{\theta}=O\left(\langle\xi\rangle^{-N}\langle w\rangle^{-N}\right)$ for any $N$, and the fact that $L$-integrations by parts allow one to gain any $\langle\zeta\rangle^{-N}\langle\xi\rangle^{N}$ factor, one sees that the corresponding contribution to (2.1.15) is in $\widetilde{H S}_{p+q, \text { loc }}^{-N, 0}$ for any $N$, as well as the action of $\left\langle D_{x}+\xi\right\rangle^{\lambda}\langle\xi\rangle^{-\lambda}$ on on that function for any $\lambda$. It follows from (2.1.13) and from (ii) of Proposition 2.1.6 that

$$
A B=\theta_{1} \mathrm{Op}\left[\theta_{1}^{2} a_{\chi} b_{\chi}\right] \theta_{1}
$$

modulo an operator $C$ satisfying the conclusion of the theorem. Since $A B=\theta A B \theta$, we may write as well, modulo such a $C, A B=\theta \mathrm{Op}\left[a_{\chi} b_{\chi}\right] \theta$. Write $a_{\chi} b_{\chi}=(a b)_{\chi}-r$ with

$$
r=\left[\left(a_{\chi} b_{\chi}\right)_{\chi}-\left(a_{\chi} b_{\chi}\right)\right]+\left(\left(a-a_{\chi}\right) b_{\chi}+\left(a_{\chi}\left(b-b_{\chi}\right)\right)_{\chi} .\right.
$$


By (iii) of proposition 2.1.6 and (2.1.10), $r$ belongs to $\widetilde{H S}_{p+q \text {,loc }}^{m+m^{\prime}-1, \nu+M_{0}}$ if $p>0, q>0$ and to $\widetilde{H S}_{p+q, \text { loc }}^{m+m^{\prime}-1, \nu+M_{0}(d+2)}$ if $p$ or $q$ is zero. The associated operator contributes to $C$ in $(2.1 .12)$. This concludes the proof in the case of homogeneous operators. The case of operators in $\Psi_{p, \text { loc }}^{m, \nu}\left(B_{\sigma}(R)\right), \Psi_{q, \text { loc }}^{m^{\prime}, \nu^{\prime}}\left(B_{\sigma}(R)\right)$ is treated similarly.

Let us finish this subsection with a similar result for transpose and adjoint.

Theorem 2.1.8 Let $m \in \mathbb{R}, \nu \in \mathbb{R}_{+}, p \in \mathbb{N}, R>0, \sigma>0$. Let $A$ be an element of the space $\widetilde{H \Psi}_{p, \nu}^{m, \nu}\left(\right.$ resp. $\left.\Psi_{p, \text { loc }}^{m, \nu}\left(B_{\sigma}(R)\right)\right)$ and let a be a symbol in $\widetilde{H S}_{p, \nu}^{m, \nu}$ (resp. $S_{p, \text { loc }}^{m, \nu}\left(B_{\sigma}(R)\right)$ ) given by (i) of Proposition 2.1.6. If $\chi$ is in $C_{0}^{\infty}(\mathbb{R})$, has small enough support, and is equal to one close to zero, if $\theta$ is in $C_{0}^{\infty}\left(\mathbb{R}^{d}\right)$ and is equal to one on a large enough compact set, then, denoting by ${ }^{t} A$ and $A^{*}$ the transpose and the adjoint of $A$ for the $L^{2}\left(\mathbb{R}^{d}, d x\right)$-scalar product,

$$
{ }^{t} A=\theta \mathrm{Op}\left(a_{\chi}^{\vee}\right) \theta+C_{1}, A^{*}=\theta \mathrm{Op}\left(\bar{a}_{\chi}\right) \theta+C_{2}
$$

where $C_{1}, C_{2}$ are in $\widetilde{H \Psi}_{p, \text { loc }}^{m-1, \nu+M_{0}(3 d+5)}$ (resp. $\left.\Psi_{p, \text { loc }}^{m-1, \nu+M_{0}(3 d+5)}\left(B_{\sigma}(R)\right)\right)$ and where $a^{\vee}(x, \xi)=$ $a(x,-\xi)$.

Proof: Because of the support assumption on the kernel of $A$ and of (iii) of Proposition 2.1.6, we know that we may write $A(\mathcal{U})=\theta \operatorname{Op}\left(a_{\chi}\right) \theta$ modulo a remainder of form $C_{1}, C_{2}$. Moreover, ${ }^{t} \mathrm{Op}\left(a_{\chi}\right)=\mathrm{Op}\left(b_{1}\right), \mathrm{Op}\left(a_{\chi}\right)^{*}=\mathrm{Op}\left(b_{2}\right)$ with

$$
\begin{aligned}
& b_{1}(x, \xi)=\frac{1}{(2 \pi)^{d}} \int e^{-i z \cdot \zeta} a_{\chi}(x-z,-\xi+\zeta) d z d \zeta \\
& b_{2}(x, \xi)=\frac{1}{(2 \pi)^{d}} \int e^{-i z \cdot \zeta} \bar{a}_{\chi}(x-z, \xi-\zeta) d z d \zeta .
\end{aligned}
$$

Because of the support of $\widehat{a_{\chi}}(\zeta,-\xi+\zeta), \widehat{\overline{a_{\chi}}}(\zeta, \xi-\zeta)$, we may insert a cut-off $\chi_{1}(\zeta /\langle\xi\rangle)$ inside the integrals in (2.1.19) if $\chi_{1} \equiv 1$ close to zero, Supp $\chi_{1}$ small enough. Then

$$
b_{1}(x, \xi)-a_{\chi}(x,-\xi)=\frac{1}{(2 \pi)^{d}} \int e^{-i z \cdot \zeta} \chi_{1}(\zeta /\langle\xi\rangle)\left[a_{\chi}(x-z,-\xi+\zeta)-a_{\chi}(x,-\xi)\right] d z d \zeta .
$$

Since the term between brackets vanishes at $(z=0, \zeta=0)$, we see, performing $d+1$ integrations by parts using $L=\frac{1-i\langle\xi\rangle^{2} z \cdot \nabla_{\zeta}}{1+\langle\xi\rangle^{2}|z|^{2}}$ that $b_{1}(x, \xi)-a_{\chi}(x,-\xi)$ may be written as $c_{\chi_{1}}(x, \xi)$ for some symbol $c$ in $\widetilde{H S}_{p, \text { loc }}^{m-1, \nu+M_{0}(d+3)}$ (resp. $\Psi_{p, \text { loc }}^{m-1, \nu+M_{0}(d+3)}\left(B_{\sigma}(R)\right)$ ). Using (ii) of Proposition 2.1.6, we get that $C_{1}=\operatorname{Op}\left(c_{\chi_{1}}\right)$ satisfies the conclusion of the theorem. One treats in the same way the case of the adjoint.

\subsection{Principal symbols}

In this subsection, we shall define principal symbols for para-differential operators on the manifold $X$, and use them to study commutators between such operators. We first study the action 
of a change of variables on local operators. If $X, X^{\prime}$ are two manifolds and $\kappa: X \rightarrow X^{\prime}$ is a diffeomorphism, we denote by $\tau_{\kappa}: T^{*} X \rightarrow T^{*} X^{\prime}$ the canonical transformation induced by $\kappa$. In local coordinates

$$
\tau_{\kappa}:(x, \xi) \rightarrow \tau_{\kappa}(x, \xi)=\left(\kappa(x),{ }^{t} d \kappa(x)^{-1} \xi\right) .
$$

We denote by $\kappa^{*}: C_{0}^{\infty}\left(X^{\prime}\right) \rightarrow C_{0}^{\infty}(X)$ the map defined by $\kappa^{*} u=u \circ \kappa$.

Proposition 2.2.1 Let $m \in \mathbb{R}, \nu \in \mathbb{R}_{+}, R>0, \sigma>0, V, \tilde{V}$ be two open sets of $\mathbb{R}^{d}, \kappa: V \rightarrow \tilde{V}$ be a $C^{\infty}$-diffeomorphism, let $\theta, \theta^{\prime}, \tilde{\theta}, \tilde{\theta}^{\prime}$ be in $C_{0}^{\infty}(V)$ with $\theta \tilde{\theta}=\theta, \theta^{\prime} \tilde{\theta}^{\prime}=\theta^{\prime}$. Let $A(\mathcal{U})$ (resp. $A(U)$ ) be an element of $\widetilde{H \Psi} \Psi_{p, \nu \text { loc }}^{m, \nu}$ (resp. $\Psi_{p, \text { loc }}^{m, \nu}\left(B_{\sigma}(R)\right)$ ). Then $B=\left(\kappa^{-1}\right)^{*} \theta A \theta^{\prime} \kappa^{*}$ is in $\widetilde{H \Psi}_{p, \text { loc }}^{m, \nu}$ (resp. $\left.\Psi_{p, \text { loc }}^{m, \nu}\left(B_{\sigma}(R)\right)\right)$. Moreover, if $\chi_{1} \in C_{0}^{\infty}(\mathbb{R})$ equals one close to zero and has small enough support, if a is the symbol in $\widetilde{H S}_{p, \mathrm{loc}}^{m, \nu}\left(\right.$ resp $S_{p, \mathrm{loc}}^{m, \nu}\left(B_{\sigma}(R)\right)$ ) associated to $A$ by (i) of Proposition 2.1.6, we may write

$$
B=\left(\tilde{\theta} \circ \kappa^{-1}\right) \operatorname{Op}\left[\left(\theta \theta^{\prime} a \circ \tau_{\kappa}^{-1}\right)_{\chi_{1}}\right]\left(\tilde{\theta}^{\prime} \circ \kappa^{-1}\right)+C
$$

where $C$ is in $\widetilde{H \Psi}_{p, \text { loc }}^{m-1, \nu+M_{0}(3 d+4)}$ (resp. $\left.\Psi_{p, \text { loc }}^{m-1, \nu+M_{0}(3 d+4)}\left(B_{\sigma}(R)\right)\right)$.

Since $\theta^{\prime} \kappa^{*}=\theta^{\prime} \kappa^{*}\left(\tilde{\theta}^{\prime} \circ \kappa^{-1}\right)$, this operator preserves Sobolev spaces. The characterization of $\widetilde{H \Psi}_{p}^{m, \nu}$ and $\Psi_{p}^{m, \nu}\left(B_{\sigma}(R)\right)$ in terms of estimates of type (1.2.9), (1.2.18), (1.2.19) thus shows that $B$ is in the indicated space of operators. We just have to show the statements concerning symbols.

Lemma 2.2.2 (i) Let $W$ be a neighborhood of the diagonal in $X \times X$. Let $\left(\theta_{i}\right)_{i \in I}$ be a smooth partition of unity on $X$ such that $\theta_{i}(x) \theta_{j}(y) \neq 0$ implies $(x, y) \in W$. Let $J=\{(i, j) \in I \times$ $\left.I ; \operatorname{Supp} \theta_{i} \cap \operatorname{Supp} \theta_{j} \neq \emptyset\right\}$. For any $A$ in $\widetilde{H \Psi}_{p}^{m, \nu}$ (resp. $\Psi_{p}^{m, \nu}\left(B_{\sigma}(R)\right)$ ), $A-\sum_{(i, j) \in J} \theta_{i} A \theta_{j}$ is in $\widetilde{H \Psi}_{p}^{m-r, \nu+r M_{0}}$ (resp. $\left.\Psi_{p}^{m-r, \nu+r M_{0}}\left(B_{\sigma}(R)\right)\right)$ for any $r \geq 0$.

(ii) Let $W$ be a neighborhood of the diagonal in $\mathbb{R}^{d} \times \mathbb{R}^{d}, K$ a compact subset of $\mathbb{R}^{d}, \chi \in$ $C_{0}^{\infty}\left(\mathbb{R}^{d}\right), \chi \equiv 1$ close to zero, with small enough support. Let $\left(\theta_{i}\right)_{i \in I}$ be a smooth partition of unity on a neighborhood of $K$. For any $A$ in $\widetilde{H \Psi}_{p, \text { loc }}^{m, \nu}$ (resp. $\Psi_{p, \text { loc }}^{m, \nu}\left(B_{\sigma}(R)\right)$ ) with support in $K \times K$, there is a symbol a in $\widetilde{H S}_{p, \nu \text { loc }}^{m, \nu}\left(\right.$ resp. $\left.S_{p, \text { loc }}^{m, \nu}\left(B_{\sigma}(R)\right)\right)$, compactly supported in $x$, such that $A-\sum_{(i, j) \in J} \theta_{i} \mathrm{Op}\left(a_{\chi}\right) \theta_{j}$ is in $\widetilde{H \Psi}_{p \text {,loc }}^{m-r, \nu+M_{0}(r+2 d+2)}$ (resp. $\Psi_{p}^{m-r, \nu+M_{0}(r+2 d+2)}\left(B_{\sigma}(R)\right)$ ) for any $r \geq 0$.

Proof: (i) We notice that if $\theta_{1}, \theta_{2}$ are smooth functions with disjoint support, $\theta_{1} A \theta_{2}$ is in $\widetilde{H \Psi}_{p}^{m-r, \nu+r M_{0}}$ (resp. $\left.\Psi_{p}^{m-r, \nu+r M_{0}}\left(B_{\sigma}(R)\right)\right)$ for any $r \geq 0$ : actually, if $\tilde{\theta}$ is in $C^{\infty}(X)$ and $\tilde{\theta} \theta_{1}=\theta_{1}$, $\tilde{\theta} \theta_{2}=0$, we may write for any $r \in \mathbb{N}$

$$
\theta_{1} A \theta_{2}=\underbrace{\operatorname{Ad}_{\tilde{\theta}} \cdots \operatorname{Ad}_{\tilde{\theta}}}_{r}\left(\theta_{1} A \theta_{2}\right) .
$$


We just have to apply the characterization of $\widetilde{H \Psi}_{p}^{m, \nu}$ (resp. $\left.\Psi_{p}^{m, \nu}\left(B_{\sigma}(R)\right)\right)$ to get the conclusion.

(ii) The same proof as above, together with the fact that $A$ is supported in $K \times K$, shows that $A-\sum_{(i, j) \in J} \theta_{i} A \theta_{j}$ is in the wanted space. By (i), (ii), (iii) of Proposition 2.1.6, there is a symbol $a$ in $\widetilde{H \Psi}_{p, \text { loc }}^{m, \nu}\left(\right.$ resp. $\left.\Psi_{p, \text { loc }}^{m, \nu}\left(B_{\sigma}(R)\right)\right)$ such that $\theta_{i} A \theta_{j}-\theta_{i} \mathrm{Op}\left(a_{\chi}\right) \theta_{j}$ belongs to $\widetilde{H \Psi}_{p, \text { loc }}^{m-r, \nu+M_{0}(r+2 d+2)}$ (resp. $\left.\Psi_{p, \text { loc }}^{m-r, \nu+M_{0}(r+2 d+2)}\left(B_{\sigma}(R)\right)\right)$. We may assume moreover that $a$ is compactly supported in a neighborhood of $K$. This concludes the proof.

Lemma 2.2.3 Let $\kappa$ be as in the statement of proposition 2.2.1. Let a be in $\widetilde{H S}_{p, \text { loc }}^{m, \nu}$ (resp. $\left.S_{p, \text { loc }}^{m, \nu}\left(B_{\sigma}(R)\right)\right)$, compactly supported in $x$ in a compact subset of $V$. Let $\chi \in C_{0}^{\infty}\left(\mathbb{R}^{d}\right), \chi \equiv 1$ close to zero, with small enough support. Let $(x, z) \rightarrow \gamma(x, z)$ be a smooth function, such that $(x, y) \rightarrow \gamma(x, x-y)$ is compactly supported in $\tilde{V} \times \tilde{V}$, and such that $(x, z) \rightarrow \gamma(x, z)$ is supported for $|z| \leq \rho \ll 1$. Define for $\mathcal{U}=\left(U_{1}, \ldots, U_{p}\right) \in C^{\infty}(X)^{p}$ (resp. for $U \in B_{\sigma}(R)$ )

$$
b(\mathcal{U}, x, \xi)=\frac{1}{(2 \pi)^{d}} \int e^{i\left[\kappa^{-1}(x)-\kappa^{-1}(x-z)\right] \zeta-i z \cdot \xi} \gamma(x, z) a_{\chi}\left(\mathcal{U}, \kappa^{-1}(x), \zeta\right) d z d \zeta
$$

(resp. $b(U, x, \xi)$ given by the same formula with $\mathcal{U}$ replaced by $U)$. Let $\Gamma \in C_{0}^{\infty}(\mathbb{R}-\{0\})$ be equal to one on a large enough compact subset of $\mathbb{R}-\{0\}$. Then one may write

$$
\begin{aligned}
b(\mathcal{U}, x, \xi)=\frac{1}{(2 \pi)^{d}} \int & e^{i\left[\left(x-x^{\prime}\right) \cdot \eta^{\prime}+z \cdot\left(\zeta^{\prime}-\xi\right)+\left(\kappa^{-1}\left(x^{\prime}\right)-x^{\prime \prime}\right) \cdot \eta^{\prime \prime}\right]} \\
& \times c\left(\mathcal{U}, x^{\prime}, x^{\prime \prime}, z, \eta^{\prime}, \eta^{\prime \prime}, \zeta^{\prime}, \xi\right) d x^{\prime} d x^{\prime \prime} d z d \eta^{\prime} d \eta^{\prime \prime} d \zeta^{\prime}+b_{1}(\mathcal{U}, x, \xi)
\end{aligned}
$$

where, for any $\lambda \in \mathbb{R},\left\langle D_{x}+\xi\right\rangle^{\lambda}\langle\xi\rangle^{-\lambda} b_{1}$ is in $H \Psi_{p, \text { loc }}^{m-r, \nu}$ for all $r \geq 0$ (resp. $b(U, x, \xi)$ may be written as (2.2.4) with $\mathcal{U}$ replaced by $U$ and $\left\langle D_{x}+\xi\right\rangle^{\lambda}\langle\xi\rangle^{-\lambda} b_{1}$ belonging to $\Psi_{p, \text { loc }}^{m-r, \nu}\left(B_{\sigma}(R)\right)$ for any $r \geq 0, \lambda \in \mathbb{R})$, and where $c$ is a function supported for

$$
\left|\eta^{\prime \prime}\right| \ll\left\langle\zeta^{\prime}\right\rangle \sim\langle\xi\rangle \sim\left\langle\xi+\eta^{\prime}\right\rangle
$$

and satisfies estimates

$$
\begin{aligned}
& \left|\partial_{x^{\prime}}^{\alpha^{\prime}} \partial_{x^{\prime \prime}}^{\alpha^{\prime \prime}} \partial_{z}^{\delta} \partial_{\xi}^{\beta} \partial_{\eta^{\prime}}^{\beta^{\prime}} \partial_{\eta^{\prime \prime}}^{\beta^{\prime \prime}} \partial_{\zeta^{\prime}}^{\gamma} c\left(\Pi_{n^{\prime}} \mathcal{U}, x^{\prime}, x^{\prime \prime}, z, \eta^{\prime}, \eta^{\prime \prime}, \zeta^{\prime}, \xi\right)\right| \\
& \quad \leq C\langle\xi\rangle^{m-|\beta|-\left|\beta^{\prime}\right|-\left|\beta^{\prime \prime}\right|-|\gamma|}\left|n^{\prime}\right|^{\nu(p)+\nu+M_{0}\left[\left|\alpha^{\prime}\right|+\left|\alpha^{\prime \prime}\right|+|\delta|+|\gamma|+d+1\right]}\left(1+\frac{\left|n^{\prime}\right|}{\langle\xi\rangle}\right)^{-N} \prod_{1}^{p}\left\|u_{\ell}\right\|_{L^{2}}
\end{aligned}
$$

resp. c satisfies the estimates

$$
\begin{aligned}
\left|\partial_{x^{\prime}}^{\alpha^{\prime}} \partial_{x^{\prime \prime}}^{\alpha^{\prime \prime}} \partial_{z}^{\delta} \partial_{\xi}^{\beta} \partial_{\eta^{\prime}}^{\beta^{\prime}} \partial_{\eta^{\prime \prime}}^{\beta^{\prime \prime}} \partial_{\zeta^{\prime}}^{\gamma} c\left(U, x^{\prime}, x^{\prime \prime}, z, \eta^{\prime}, \eta^{\prime \prime}, \zeta^{\prime}, \xi\right)\right| \\
\quad \leq C\langle\xi\rangle^{m-|\beta|-\left|\beta^{\prime}\right|-\left|\beta^{\prime \prime}\right|-|\gamma|+\left(\nu(p)+\nu+M_{0}\left[\left|\alpha^{\prime}\right|+\left|\alpha^{\prime \prime}\right|+|\delta|+|\gamma|+d+1\right]-\sigma\right)_{+}}\|U\|_{H^{\sigma}}^{p}
\end{aligned}
$$

and the estimate

$$
\begin{aligned}
\left|\partial_{x^{\prime}}^{\alpha^{\prime}} \partial_{x^{\prime \prime}}^{\alpha^{\prime \prime}} \partial_{z}^{\delta} \partial_{\xi}^{\beta} \partial_{\eta^{\prime}}^{\beta^{\prime}} \partial_{\eta^{\prime \prime}}^{\beta^{\prime \prime}} \partial_{\zeta^{\prime}}^{\gamma}\left[\partial_{U} c\left(U, x^{\prime}, x^{\prime \prime}, z, \eta^{\prime}, \eta^{\prime \prime}, \zeta^{\prime}, \xi\right) \cdot H\right]\right| \\
\quad \leq C\langle\xi\rangle^{m-|\beta|-\left|\beta^{\prime}\right|-\left|\beta^{\prime \prime}\right|-|\gamma|+\left(\nu(p)+\nu+M_{0}\left[\left|\alpha^{\prime}\right|+\left|\alpha^{\prime \prime}\right|+|\delta|+|\gamma|+d+1\right]-\sigma^{\prime}\right)_{+}}\|U\|_{H^{\sigma}}^{p-1}\|H\|_{H^{\sigma^{\prime}}}
\end{aligned}
$$


for any $\sigma^{\prime} \in[-\sigma, \sigma]$, any $H$ in $H^{\sigma^{\prime}}$.

Moreover, the restriction of $c$ at $z=0$ is given by

$$
\begin{aligned}
\left.c\right|_{z=0}=\frac{1}{(2 \pi)^{d}} \gamma\left(x^{\prime}, 0\right)\left|\operatorname{det} \kappa\left(\kappa^{-1}\left(x^{\prime}\right)\right)\right| \chi\left(\eta^{\prime \prime} /\left\langle{ }^{t} d \kappa\left(\kappa^{-1}\left(x^{\prime}\right)\right) \cdot \zeta^{\prime}\right\rangle\right) \\
\times \Gamma\left(\frac{\left\langle\zeta^{\prime}\right\rangle}{\langle\xi\rangle}\right) \Gamma\left(\frac{\left\langle\zeta^{\prime}\right\rangle}{\left\langle\xi+\eta^{\prime}\right\rangle}\right) a\left(\mathcal{U}, x^{\prime \prime},{ }^{t} d \kappa\left(\kappa^{-1}\left(x^{\prime}\right)\right) \cdot \zeta^{\prime}\right)
\end{aligned}
$$

(resp. by the same formula with $\mathcal{U}$ replaced by $U$ ).

Proof: Let us prove the lemma in the case $a \in \widetilde{H S}_{p, \text { loc }}^{m, \nu}$. We may write the definition (2.2.3) of $b$

$$
\begin{array}{r}
b(\mathcal{U}, x, \xi)=\frac{1}{(2 \pi)^{3 d}} \int e^{i\left[\left(x-x^{\prime}\right) \eta^{\prime}+\left(\kappa^{-1}\left(x^{\prime}\right)-\kappa^{-1}\left(x^{\prime}-z\right)\right) \zeta+\left(\kappa^{-1}\left(x^{\prime}\right)-x^{\prime \prime}\right) \eta^{\prime \prime}-z \xi\right]} \gamma\left(x^{\prime}, z\right) \chi\left(\eta^{\prime \prime} /\langle\zeta\rangle\right) \\
\times a\left(\mathcal{U}, x^{\prime \prime}, \zeta\right) d x^{\prime} d x^{\prime \prime} d z d \eta^{\prime} d \eta^{\prime \prime} d \zeta
\end{array}
$$

On the support of $\gamma\left(x^{\prime}, z\right) \Gamma\left(\left\langle\nabla_{x^{\prime}}\left(\kappa^{-1}\left(x^{\prime}-z\right) \cdot \zeta\right)\right\rangle /\langle\xi\rangle\right)$, the quotient $\langle\zeta\rangle /\langle\xi\rangle$ stays between two uniform constants (since $x^{\prime}-z$ stays in a compact subset of $\tilde{V}$ ), and the function $\Gamma_{1}\left(x^{\prime}-z, \zeta, \xi\right)=$ $(1-\Gamma)\left(\left\langle\nabla_{x^{\prime}}\left(\kappa^{-1}\left(x^{\prime}-z\right) \cdot \zeta\right)\right\rangle /\langle\xi\rangle\right)$ satisfies

$$
\partial_{x^{\prime}}^{\alpha^{\prime}} \partial_{z}^{\delta} \Gamma_{1}\left(x^{\prime}-z, \zeta, \xi\right)=O(1) .
$$

Define $b_{1}^{\prime}$ as the function given by formula (2.2.10) with $\Gamma_{1}$ inserted inside the integral, and define

$$
L_{1}=\frac{1-i\left(\nabla_{x^{\prime}}\left(\kappa^{-1}\left(x^{\prime}-z\right) \cdot \zeta\right)-\xi\right) \cdot \nabla_{z}}{1+\left|\nabla_{x^{\prime}}\left(\kappa^{-1}\left(x^{\prime}-z\right) \cdot \zeta\right)-\xi\right|^{2}} .
$$

Performing integrations by parts using $L_{1}$, and denoting by $\Phi$ the phase function in (2.2.10), we write

$$
\begin{aligned}
b_{1}^{\prime}(\mathcal{U}, x, \xi)= & \frac{1}{(2 \pi)^{3 d}} \int e^{i \Phi\left(x, x^{\prime}, x^{\prime \prime}, z, \eta^{\prime}, \eta^{\prime \prime}, \zeta, \xi\right)} \\
& \times\left({ }^{t} L_{1}\right)^{N}\left[\gamma\left(x^{\prime}, z\right) \chi\left(\eta^{\prime \prime} /\langle\zeta\rangle\right) \Gamma_{1}\left(x^{\prime}-z, \zeta, \xi\right) a\left(\mathcal{U}, x^{\prime \prime}, \zeta\right)\right] d x^{\prime} d x^{\prime \prime} d z d \eta^{\prime} d \eta^{\prime \prime} d \zeta
\end{aligned}
$$

We note that, if the domain over which $\Gamma \equiv 1$ is large enough, on the support of $\Gamma_{1}=1-\Gamma$, either $\langle\zeta\rangle \gg\langle\xi\rangle$ or $\langle\zeta\rangle \ll\langle\xi\rangle$, so that every $L_{1}$-integration by parts will provide a gain of $(1+|\zeta|+|\xi|)^{-1}$. Consequently, the symbol in the integral in (2.2.12) satisfies the same estimates as $a$, as well as its derivatives, with a gain in $(1+|\zeta|+|\xi|)^{-N}$. If we insert inside the integral another cut-off $\omega\left(\eta^{\prime} /\left(1+\xi^{2}+\zeta^{2}\right)^{1 / 2}\right)$, with $\omega$ in $C_{0}^{\infty}\left(\mathbb{R}^{d}\right)$, and use that the integrand is compactly supported in $\left(x^{\prime}, x^{\prime \prime}, z\right)$, we obtain for the corresponding contribution to $b_{1}^{\prime}$ a bound in

$$
C_{N}\langle\xi\rangle^{-N^{\prime}}\left|n^{\prime}\right|^{\nu(p)+\nu}\left(1+\frac{\left|n^{\prime}\right|}{\langle\xi\rangle}\right)^{-N^{\prime}}
$$

if $N \gg N^{\prime}$. Similar estimates hold for derivatives. Moreover, if we make act on $b_{1}^{\prime}$ the operator $\left\langle D_{x}+\xi\right\rangle^{\lambda}\langle\xi\rangle^{-\lambda}$, and use that $\left\langle\eta^{\prime}+\xi\right\rangle$ is controlled by the gain coming from the integrations by parts, we still get a bound of type (2.2.13). 
Consider now the contribution to $b_{1}^{\prime}$ given by (2.2.12) in which we insert $(1-\omega)\left(\eta^{\prime} /\left(1+\xi^{2}+\zeta^{2}\right)^{1 / 2}\right)$, with $\omega \equiv 1$ on a large enough compact set. We consider

$$
L_{2}=\frac{1-i\left(\nabla_{x^{\prime}}\left(\kappa^{-1}\left(x^{\prime}\right) \cdot\left(\eta^{\prime \prime}+\zeta\right)\right)-\left(\eta^{\prime}+\xi\right)\right) \cdot\left(\nabla_{x^{\prime}}+\nabla_{z}\right)}{1+\left|\nabla_{x^{\prime}}\left(\kappa^{-1}\left(x^{\prime}\right) \cdot\left(\eta^{\prime \prime}+\zeta\right)\right)-\left(\eta^{\prime}+\xi\right)\right|^{2}}
$$

and perform $N$ integrations by parts using $L_{2}$. We get

$$
\begin{aligned}
\frac{1}{(2 \pi)^{3 d}} \int e^{i \Phi\left(x, x^{\prime}, x^{\prime \prime}, z, \eta^{\prime}, \eta^{\prime \prime}, \zeta, \xi\right)}\left({ }^{t} L_{2}\right)^{N}\left[(1-\omega)\left(\frac{\eta^{\prime}}{\left(1+\xi^{2}+\zeta^{2}\right)^{1 / 2}}\right)\right. \\
\left.\quad \times\left(\left({ }^{t} L_{1}\right)^{N}\left[\gamma\left(x^{\prime}, z\right) \chi\left(\eta^{\prime \prime} /\langle\zeta\rangle\right) \Gamma_{1}\left(x^{\prime}-z, \zeta, \xi\right) a\left(\mathcal{U}, x^{\prime \prime}, \zeta\right)\right]\right)\right] d x^{\prime} d x^{\prime \prime} d z d \eta^{\prime} d \eta^{\prime \prime} d \zeta
\end{aligned}
$$

Since on the support of the integrand, $\left|\eta^{\prime \prime}\right| \ll\langle\zeta\rangle,\left|\eta^{\prime}\right| \gg(1+|\xi|+|\zeta|)$, the integrations by parts gain a $\left(1+\left|\eta^{\prime}\right|+\left|\eta^{\prime \prime}\right|+|\zeta|+|\xi|\right)^{-N}$ factor. We obtain again a quantity bounded by (2.2.13), with similar estimates for the derivatives or for the action of $\left\langle D_{x}+\xi\right\rangle^{\lambda}\langle\xi\rangle^{-\lambda}$ for any $\lambda$. Consequently, $b_{1}^{\prime}$ contributes to $b_{1}$ in (2.2.4). Let us study next $b_{1}^{\prime \prime}$, which is given by $(2.2 .10)$ where we insert the cut-off $\Gamma\left(\left\langle\nabla_{x^{\prime}}\left(\kappa^{-1}\left(x^{\prime}-z\right) \cdot \zeta\right)\right\rangle /\langle\xi\rangle\right)$. In particular, $\langle\zeta\rangle \sim\langle\xi\rangle$ on the domain of integration. We decompose $b_{1}^{\prime \prime}=b_{0}+b_{1}^{\prime \prime \prime}$ with

$$
\begin{aligned}
b_{0}(\mathcal{U}, x, \xi) & =\frac{1}{(2 \pi)^{3 d}} \int e^{i \Phi\left(x, x^{\prime}, x^{\prime \prime}, z, \eta^{\prime}, \eta^{\prime \prime}, \zeta, \xi\right)} \Gamma\left(\frac{\left\langle\nabla_{x^{\prime}}\left(\kappa^{-1}\left(x^{\prime}\right) \cdot \zeta\right)\right\rangle}{\left\langle\xi+\eta^{\prime}\right\rangle}\right) \\
& \times \gamma\left(x^{\prime}, z\right) \chi\left(\eta^{\prime \prime} /\langle\zeta\rangle\right) \Gamma\left(\frac{\left\langle\nabla_{x^{\prime}}\left(\kappa^{-1}\left(x^{\prime}-z\right) \cdot \zeta\right)\right\rangle}{\langle\xi\rangle}\right) a\left(\mathcal{U}, x^{\prime \prime}, \zeta\right) d x^{\prime} d x^{\prime \prime} d z d \eta^{\prime} d \eta^{\prime \prime} d \zeta
\end{aligned}
$$

and $b_{1}^{\prime \prime \prime}$ being given by the same integral, with the first $\Gamma$ replaced by $1-\Gamma$. We study first $b_{1}^{\prime \prime \prime}$, performing $N$ integrations by parts using $L_{2}$. We get

$$
\begin{aligned}
& \frac{1}{(2 \pi)^{3 d}} \int e^{i \Phi\left(x, x^{\prime}, x^{\prime \prime}, z, \eta^{\prime}, \eta^{\prime \prime}, \zeta, \xi\right) t} L_{2}^{N}\left[(1-\Gamma)\left(\frac{\left\langle\nabla_{x^{\prime}}\left(\kappa^{-1}\left(x^{\prime}\right) \cdot \zeta\right)\right\rangle}{\left\langle\xi+\eta^{\prime}\right\rangle}\right)\right. \\
& \left.\quad \times \gamma\left(x^{\prime}, z\right) \chi\left(\eta^{\prime \prime} /\langle\zeta\rangle\right) \Gamma\left(\frac{\left\langle\nabla_{x^{\prime}}\left(\kappa^{-1}\left(x^{\prime}-z\right) \cdot \zeta\right)\right\rangle}{\langle\xi\rangle}\right) a\left(\mathcal{U}, x^{\prime \prime}, \zeta\right)\right] d x^{\prime} d x^{\prime \prime} d z d \eta^{\prime} d \eta^{\prime \prime} d \zeta
\end{aligned}
$$

On the support of the domain of integration, $\left|\eta^{\prime \prime}\right| \ll\langle\zeta\rangle,\langle\zeta\rangle \sim\langle\xi\rangle$ and either $\langle\zeta\rangle \ll\left\langle\xi+\eta^{\prime}\right\rangle$ or $\langle\zeta\rangle \gg\left\langle\xi+\eta^{\prime}\right\rangle$. This implies that the integrations by parts gain a factor $\left(1+|\zeta|+\left|\xi+\eta^{\prime}\right|\right)^{-N}$, and that (2.2.15) has a bound of form (2.2.13), as well as the action of $\left\langle D_{x}+\xi\right\rangle^{\lambda}\langle\xi\rangle^{-\lambda}$ on this integral. Since derivatives are estimated in the same way, we conclude that $b_{1}^{\prime \prime \prime}$ contributes to $b_{1}$ in $(2.2 .4)$.

Finally, we are left with showing that $b_{0}$ may be written as the first term in the right hand side of (2.2.4). Since the cut-off $\gamma$ is supported for $\left|z^{\prime}\right| \leq \rho \ll 1$, we may write, if $\rho$ is small enough, $\kappa^{-1}\left(x^{\prime}\right)-\kappa^{-1}\left(x^{\prime}-z\right)={ }^{t} B\left(x^{\prime}, z\right)^{-1} z$, where $B\left(x^{\prime}, 0\right)={ }^{t} d \kappa\left(\kappa^{-1}\left(x^{\prime}\right)\right)$ and $B\left(x^{\prime}, z\right)$ is an invertible matrix for any $z$ with $|z| \ll \rho$. Setting $\zeta=B\left(x^{\prime}, z\right) \cdot \zeta^{\prime}$ in (2.2.14), we rewrite

$$
\begin{aligned}
b_{0}(\mathcal{U}, x, \xi)=\frac{1}{(2 \pi)^{2 d}} \int e^{i\left[\left(x-x^{\prime}\right) \cdot \eta^{\prime}+z \cdot\left(\zeta^{\prime}-\xi\right)+\left(\kappa^{-1}\left(x^{\prime}\right)-x^{\prime \prime}\right) \cdot \eta^{\prime \prime}\right]} & \\
& \times c\left(\mathcal{U}, x^{\prime}, x^{\prime \prime}, z, \eta^{\prime}, \eta^{\prime \prime}, \zeta^{\prime}, \xi\right) d x^{\prime} d x^{\prime \prime} d z d \eta^{\prime} d \eta^{\prime \prime} d \zeta^{\prime}
\end{aligned}
$$


with

$$
\begin{aligned}
c\left(\mathcal{U}, x^{\prime}, x^{\prime \prime}, z, \eta^{\prime}, \eta^{\prime \prime}, \zeta^{\prime}, \xi\right)= & \frac{\tilde{\gamma}\left(x^{\prime}, z\right)}{(2 \pi)^{d}} \chi\left(\frac{\eta^{\prime \prime}}{\left\langle B\left(x^{\prime}, z\right) \cdot \zeta^{\prime}\right\rangle}\right) \Gamma\left(\frac{\left\langle\left(\nabla_{x^{\prime}}\left(\kappa^{-1}\left(x^{\prime}\right)\right) \cdot B\left(x^{\prime}, z\right) \cdot \zeta^{\prime}\right)\right\rangle}{\left\langle\xi+\eta^{\prime}\right\rangle}\right) \\
& \times \Gamma\left(\frac{\left\langle\nabla_{x^{\prime}}\left(\kappa^{-1}\left(x^{\prime}-z\right)\right) \cdot\left(B\left(x^{\prime}, z\right) \cdot \zeta^{\prime}\right)\right\rangle}{\langle\xi\rangle}\right) a\left(\mathcal{U}, x^{\prime \prime}, B\left(x^{\prime}, z\right) \cdot \zeta^{\prime}\right)
\end{aligned}
$$

for another smooth function $\tilde{\gamma}$, compactly supported in $\left(x^{\prime}, z\right)$ in $|z| \leq \rho \ll 1$, such that

$$
\tilde{\gamma}\left(x^{\prime}, 0\right)=\gamma\left(x^{\prime}, 0\right)\left|\operatorname{det} d \kappa\left(\kappa^{-1}\left(x^{\prime}\right)\right)\right| .
$$

We note that (2.2.5) and estimates (2.2.6) hold true, and that $\left.c\right|_{z=0}$ is given by (2.2.9). This concludes the proof of Lemma 2.2.3, as the case of symbols in $S_{p, \text { loc }}^{m, \nu}\left(B_{\sigma}(R)\right)$ is identical.

Proof of proposition 2.2.1: We consider $A$ in $\widetilde{H \Psi}_{p, \nu}^{m, \nu}$. According to Lemma 2.2.2, we may find a $C_{0}^{\infty}\left(\mathbb{R}^{d} \times \mathbb{R}^{d}\right)$-function, $(x, z) \rightarrow \gamma_{1}(x, z)$, supported for $|z| \leq \rho \ll 1$, satisfying $\theta \theta^{\prime}(x) \gamma_{1}(x, 0)=$ $\theta \theta^{\prime}(x)$ and such that $A(\mathcal{U})$ is given, up to an operator in in $\widetilde{H \Psi}_{p, \text { loc }}^{m-r, \nu+M_{0}(r+2 d+2)}$ for any $r$, by the operator $\tilde{A}(\mathcal{U})$ with kernel

$$
K(\mathcal{U}, x, y)=\frac{1}{(2 \pi)^{d}} \int e^{i(x-y) \cdot \xi} \gamma_{1}(x, x-y) a_{\chi}(\mathcal{U}, x, \xi) d \xi
$$

where $a$ is an element of $\widetilde{H S}_{p, \nu}^{m, \nu}$, compactly supported in $x$. The distribution kernel of the operator $\left(\kappa^{-1}\right)^{*} \theta \tilde{A} \theta^{\prime} \kappa^{*}$ will be

$$
\tilde{\theta} \circ \kappa^{-1}(x) \frac{1}{(2 \pi)^{d}} \int e^{i\left(\kappa^{-1}(x)-\kappa^{-1}(y)\right) \cdot \xi} \gamma(x, x-y) a_{\chi}\left(\mathcal{U}, \kappa^{-1}(x), \xi\right) d \xi \tilde{\theta}^{\prime} \circ \kappa^{-1}(y)
$$

where

$$
\gamma(x, z)=\theta \circ \kappa^{-1}(x) \gamma_{1}\left(\kappa^{-1}(x), \kappa^{-1}(x)-\kappa^{-1}(x-z)\right) \theta^{\prime} \circ \kappa^{-1}(x-z)\left|\operatorname{det} d \kappa^{-1}(x-z)\right| .
$$

Consequently, $\left(\kappa^{-1}\right)^{*} \theta \tilde{A}(\mathcal{U}) \theta^{\prime} \kappa^{*}=\left(\tilde{\theta} \circ \kappa^{-1}\right) \operatorname{Op}(b(\mathcal{U}, \cdot))\left(\tilde{\theta}^{\prime} \circ \kappa^{-1}\right)$ where $b$ is given by $(2.2 .3)$. We apply Lemma 2.2.3. By (2.2.4) and (ii) of proposition 2.1.6, we may replace $b$ by the first term in the right hand side of (2.2.4), up to an operator in $\widetilde{H \Psi}_{p, \text { loc }}^{m-r, \nu+2 M_{0}(d+1)}$ for any $r \geq 0$. Write $c=c_{0}+c_{1}$ where $c_{0}=\left.c\right|_{z=0}$ is given by (2.2.9). The contribution corresponding to $c_{0}$ to the first term in the right hand side of (2.2.4) is

$$
\begin{aligned}
& \Gamma\left(\frac{\langle\xi\rangle}{\left\langle D_{x}+\xi\right\rangle}\right)[\gamma(x, 0) \mid \operatorname{det}\left.d \kappa\left(\kappa^{-1}(x)\right) \mid a_{\chi}\left(\mathcal{U}, \kappa^{-1}(x),{ }^{t} d \kappa\left(\kappa^{-1}(x)\right) \cdot \xi\right)\right] \\
&=\Gamma\left(\frac{\langle\xi\rangle}{\left\langle D_{x}+\xi\right\rangle}\right)\left[\left(\theta \theta^{\prime}\right) \circ \kappa^{-1} a_{\chi}\left(\mathcal{U}, \kappa^{-1}(x),{ }^{t} d \kappa\left(\kappa^{-1}(x)\right) \cdot \xi\right)\right]
\end{aligned}
$$

We write

$$
\left(\theta \theta^{\prime} a_{\chi}\right) \circ \tau_{\kappa}=\left(\theta \theta^{\prime}\left(a_{\chi}-a\right)\right) \circ \tau_{\kappa}+\left[\left(\theta \theta^{\prime} a\right) \circ \tau_{\kappa}-\left(\left(\theta \theta^{\prime} a\right) \circ \tau_{\kappa}\right)_{\chi_{1}}\right]+\left(\left(\theta \theta^{\prime} a\right) \circ \tau_{\kappa}\right)_{\chi_{1}}
$$

with $\chi_{1} \in C_{0}^{\infty}\left(\mathbb{R}^{d}\right), \chi_{1} \equiv 1$ close to zero. By (iii) of Proposition 2.1.6, the first two terms in the right hand side belong to $\widetilde{H S}_{p, \text { loc }}^{m-r, \nu+r M_{0}}$ for any $r \geq 0$. If one makes act on them the spectral 
cut-off $\Gamma\left(\frac{\langle\xi\rangle}{\left\langle D_{x}+\xi\right\rangle}\right)$ of (2.2.16), one obtains elements of the same space, that stay in it if one applies $\left\langle D_{x}+\xi\right\rangle^{\lambda}\langle\xi\rangle^{-\lambda}$ for any $\lambda \in \mathbb{R}$. Consequently, since $\Gamma\left(\frac{\langle\xi\rangle}{\left\langle D_{x}+\xi\right\rangle}\right) \chi_{1}(D /\langle\xi\rangle)=\chi_{1}(D /\langle\xi\rangle)$ if Supp $\chi_{1}$ is small enough, we may write

$$
\left(\kappa^{-1}\right)^{*} \theta \tilde{A} \theta^{\prime} \kappa^{*}=\left(\tilde{\theta} \circ \kappa^{-1}\right) \operatorname{Op}\left(\left(\left(\theta \theta^{\prime} a\right) \circ \tau_{\kappa}\right)_{\chi_{1}}\right)\left(\tilde{\theta}^{\prime} \circ \kappa^{-1}\right)+\left(\tilde{\theta} \circ \kappa^{-1}\right) \operatorname{Op}(\tilde{b})\left(\tilde{\theta}^{\prime} \circ \kappa^{-1}\right)+C_{1}
$$

where $C_{1}$ contributes to $C$ in $(2.2 .2)$ and $\tilde{b}$ is given by the first term in the right hand side of (2.2.4), with $c$ replaced by $c_{1}=c-\left.c\right|_{z=0}$. We may write $c_{1}=z c_{1}^{\prime}$, where $c_{1}^{\prime}$ satisfies estimates (2.2.6), with $\nu$ replaced by $\nu+M_{0}$.

Then

$$
\begin{aligned}
\tilde{b}(\mathcal{U}, x, \xi)=\frac{1}{(2 \pi)^{2 d}} \int e^{i\left[\left(x-x^{\prime}\right) \cdot \eta^{\prime}+z \cdot\left(\zeta^{\prime}-\xi\right)+\left(\kappa^{-1}\left(x^{\prime}\right)-x^{\prime \prime}\right) \cdot \eta^{\prime \prime}\right]} & \\
& \times i\left(\partial_{\zeta^{\prime}} c_{1}^{\prime}\right)\left(\mathcal{U}, x^{\prime}, x^{\prime \prime}, z, \eta^{\prime}, \eta^{\prime \prime}, \zeta^{\prime}, \xi\right) d x^{\prime} d x^{\prime \prime} d z d \eta^{\prime} d \eta^{\prime \prime} d \zeta^{\prime}
\end{aligned}
$$

Consider the vector fields

$$
\begin{aligned}
L_{3} & =\frac{1-i\langle\xi\rangle^{2}\left(\kappa^{-1}\left(x^{\prime}\right)-x^{\prime \prime}\right) \cdot \nabla_{\eta^{\prime \prime}}}{1+\langle\xi\rangle^{2}\left|\kappa^{-1}\left(x^{\prime}\right)-x^{\prime \prime}\right|^{2}} \\
L_{4} & =\frac{1-i\langle\xi\rangle^{2}\left(x-x^{\prime}\right) \cdot \nabla_{\eta^{\prime}}}{1+\langle\xi\rangle^{2}\left|x-x^{\prime}\right|^{2}} \\
L_{5} & =\frac{1-i\langle\xi\rangle^{2}\left|n^{\prime}\right|^{-2 M_{0}} z \cdot \nabla_{\zeta^{\prime}}}{1+|z|^{2}\langle\xi\rangle^{2}\left|n^{\prime}\right|^{-2 M_{0}}} .
\end{aligned}
$$

If we perform $N$ integrations by parts using $L_{3}, L_{4}, L_{5}$, use (2.2.6) and the support condition (2.2.5), we estimate $(2.2 .17)$ by

$$
\begin{aligned}
\int\left(1+\langle\xi\rangle\left|\kappa^{-1}\left(x^{\prime}\right)-x^{\prime \prime}\right|\right)^{-N}\left(1+\langle\xi\rangle\left|x-x^{\prime}\right|\right)^{-N}\left(1+|z|\langle\xi\rangle\left|n^{\prime}\right|^{-M_{0}}\right)^{-N} \\
\times \mathbb{1}_{\left|\eta^{\prime \prime}\right| \ll\left\langle\zeta^{\prime}\right\rangle \sim\langle\xi\rangle \sim\left\langle\xi+\eta^{\prime}\right\rangle} d x^{\prime} d x^{\prime \prime} d z d \eta^{\prime} d \eta^{\prime \prime} d \zeta^{\prime} \\
\times\langle\xi\rangle^{m-1}\left|n^{\prime}\right|^{\nu(p)+\nu+M_{0}(d+3)}\left(1+\frac{\left|n^{\prime}\right|}{\langle\xi\rangle}\right)^{-N} \\
\leq C\langle\xi\rangle^{m-1}\left|n^{\prime}\right|^{\nu(p)+\nu+M_{0}(2 d+3)}\left(1+\frac{\left|n^{\prime}\right|}{\langle\xi\rangle}\right)^{-N} .
\end{aligned}
$$

Since the derivatives are estimated in the same way, we get an element of $\widetilde{H S}_{p, \text { loc }}^{m-1, \nu+M_{0}(d+2)}$, which stays in that space if we apply any $\left\langle D_{x}+\xi\right\rangle^{\lambda}\langle\xi\rangle^{-\lambda}$ because of the support condition (2.2.5). The corresponding operator is in $\widetilde{H \Psi}_{p, \text { loc }}^{m-1, \nu+M_{0}(3 d+4)}$ by (ii) of proposition 2.1.6. This concludes the proof, as the case of operators in $\Psi_{p, l o c}^{m, \nu}\left(B_{\sigma}(R)\right)$ is similar.

Let us define a principal symbol. 
Definition 2.2.4 Let $m \in \mathbb{R}, \nu \in \mathbb{R}_{+}, p \in \mathbb{N}, \sigma>0, R>0$. One denotes by $\widetilde{H S}_{p}^{m, \nu}\left(T^{*} X\right)$ (resp. $\left.S_{p}^{m, \nu}\left(B_{\sigma}(R), T^{*} X\right)\right)$ the space of maps $(\mathcal{U}, \rho)=\left(U_{1}, \ldots, U_{p}, \rho\right) \rightarrow a(\mathcal{U}, \rho)(\operatorname{resp} .(U, \rho) \rightarrow a(U, \rho))$ which are defined on $C^{\infty}(X)^{p} \times T^{*} X$ (resp. $\left.B_{\sigma}(R) \times T^{*} X\right)$, with values in $\mathbb{C}$, which are $p$-linear in $\mathcal{U}$ (resp. $C^{1}$ in $\left.U\right)$, smooth in $\rho$, and such that for any local coordinate system $\kappa$ defined on an open set $V$ of $X$, with values in $\mathbb{R}^{d}$, for any $\theta$ in $C_{0}^{\infty}(V),(\theta a) \circ \tau_{\kappa}^{-1}$ is an element of $\widetilde{H S}{ }_{p, l o c}^{m, \nu}$ (resp. $\left.S_{p, \text { loc }}^{m, \nu}\left(B_{\sigma}(R)\right)\right)$.

Definition 2.2.5 One says that an element $A$ of $\widetilde{H \Psi}_{p}^{m, \nu}$ (resp. $\left.\Psi_{p}^{m, \nu}\left(B_{\sigma}(R)\right)\right)$ admits an element a of $\widetilde{H S}{ }_{p}^{m, \nu}\left(T^{*} X\right)$ (resp. $S_{p}^{m, \nu}\left(B_{\sigma}(R), T^{*} X\right)$ ) as a principal symbol modulo $\widetilde{H \Psi}_{p}^{m-1, \nu+\ell}$ (resp. $\Psi_{p}^{m-1, \nu+\ell}\left(B_{\sigma}(R)\right)$ ) if and only if, for any $\chi$ in $C_{0}^{\infty}\left(\mathbb{R}^{d}\right), \chi \equiv 1$ close to zero, with small enough support, for any local chart $\kappa: V \subset X \rightarrow \mathbb{R}^{d}$, for any functions $\theta, \theta^{\prime}, \tilde{\theta}, \tilde{\theta}^{\prime}$ in $C_{0}^{\infty}(V)$, with $\theta \tilde{\theta}=\theta, \theta^{\prime} \tilde{\theta}^{\prime}=\theta^{\prime}$,

$$
\left(\kappa^{-1}\right)^{*} \theta A \theta^{\prime} \kappa^{*}-\left(\tilde{\theta} \circ \kappa^{-1}\right) \operatorname{Op}\left[\left(\left(\theta \theta^{\prime} a\right) \circ \tau_{\kappa}^{-1}\right)_{\chi}\right]\left(\tilde{\theta}^{\prime} \circ \kappa^{-1}\right)
$$

is in $\widetilde{H \Psi}_{p, \text { loc }}^{m-1, \nu+\ell}$ (resp. $\left.\Psi_{p, \text { loc }}^{m-1, \nu+\ell}\left(B_{\sigma}(R)\right)\right)$.

Remark: If $\ell \geq M_{0}(3 d+4)$, the above definition is coherent. Actually, consider two local coordinate systems $\kappa: V \stackrel{\sim}{\rightarrow} \tilde{V}, \kappa_{1}: W \stackrel{\sim}{\rightarrow} \tilde{W}$, where $V, W$ are non disjoint open subsets of $X$, and $\tilde{V}, \tilde{W}$ are open subsets of $\mathbb{R}^{d}$. Let $\theta, \theta^{\prime}, \tilde{\theta}, \tilde{\theta}^{\prime}$ be in $C_{0}^{\infty}(V \cap W)$, with $\theta \tilde{\theta}=\theta, \theta^{\prime} \tilde{\theta}^{\prime}=\theta^{\prime}$ and set $\mathcal{O}=\kappa(V \cap W), \mathcal{O}_{1}=\kappa_{1}(V \cap W)$. Then

$$
\begin{aligned}
\left(\kappa_{1}^{-1}\right)^{*} \theta A \theta^{\prime} \kappa_{1}^{*} & =\left(\left.\kappa \circ \kappa_{1}^{-1}\right|_{\mathcal{O}_{1}}\right)^{*}\left(\kappa^{-1}\right)^{*} \theta A \theta^{\prime} \kappa^{*}\left(\left.\kappa_{1} \circ \kappa^{-1}\right|_{\mathcal{O}}\right)^{*} \\
& =\left(\left.\kappa \circ \kappa_{1}^{-1}\right|_{\mathcal{O}_{1}}\right)^{*}\left(\tilde{\theta} \circ \kappa^{-1}\right) \operatorname{Op}\left[\left(\theta \theta^{\prime} a\right) \circ \tau_{\kappa}^{-1}\right]_{\chi}\left(\tilde{\theta}^{\prime} \circ \kappa^{-1}\right)\left(\left.\kappa_{1} \circ \kappa^{-1}\right|_{\mathcal{O}}\right)^{*}
\end{aligned}
$$

modulo $\widetilde{H \Psi}_{p \text {,loc }}^{m-1, \nu+\ell}\left(\right.$ resp. $\left.\Psi_{p, \text { loc }}^{m-1, \nu+\ell}\left(B_{\sigma}(R)\right)\right)$. By proposition 2.2.1, this is equal to

$$
\left(\tilde{\theta} \circ \kappa_{1}^{-1}\right) \operatorname{Op}\left[\left(\theta \theta^{\prime} a\right) \circ \tau_{\kappa_{1}}^{-1}\right]_{\chi_{1}}\left(\tilde{\theta}^{\prime} \circ \kappa_{1}^{-1}\right)
$$

modulo $\widetilde{H \Psi}_{p \text {, loc }}^{m-1, \nu+M_{0}(3 d+4)}$ (resp. $\Psi_{p, \text { loc }}^{m-1, \nu+M_{0}(3 d+4)}\left(B_{\sigma}(R)\right)$ ). Of course, using (iii) of Proposition 2.1.6, we may replace $\chi_{1}$ by any other function $\chi$ in $C_{0}^{\infty}\left(\mathbb{R}^{d}\right), \chi \equiv 1$ close to zero, with small enough support.

Proposition 2.2.6 Let $m \in \mathbb{R}, \nu \in \mathbb{R}_{+}, \nu^{\prime} \in \mathbb{R}_{+}, p, q \in \mathbb{N}, \sigma>0$.

(i) Every element of $\widetilde{H \Psi}_{p}^{m, \nu}$ (resp. $\Psi_{p}^{m, \nu}\left(B_{\sigma}(R)\right)$ ) has a principal symbol a in $\widetilde{H S}_{p}^{m, \nu}\left(T^{*} X\right)$ (resp. $\left.S_{p}^{m, \nu}\left(B_{\sigma}(R), T^{*} X\right)\right)$ modulo $\widetilde{H \Psi}_{p}^{m-1, \nu+M_{0}(3 d+4)}$ (resp. $\Psi_{p}^{m-1, \nu+M_{0}(3 d+4)}\left(B_{\sigma}(R)\right)$ ).

(ii) Let a be an element of $\widetilde{H S}_{p}^{m, \nu}\left(T^{*} X\right)$ (resp. $S_{p}^{m, \nu}\left(B_{\sigma}(R), T^{*} X\right)$ ). There is an operator $A$ in $\widetilde{H \Psi}_{p}^{m, \nu}$ (resp. $\left.\Psi_{p}^{m, \nu}\left(B_{\sigma}(R)\right)\right)$ whose principal symbol modulo $\widetilde{H \Psi}_{p}^{m-1, \nu+M_{0}(3 d+4)}$ (resp. $\left.\Psi_{p}^{m-1, \nu+M_{0}(3 d+4)}\left(B_{\sigma}(R)\right)\right)$ is a.

(iii) Assume $\sigma>\min (\nu(p), \nu(q))+\min \left(\nu, \nu^{\prime}\right)+M_{0}(3 d+4)$, and, when $p>0$ and $q>0$, assume moreover that (2.1.10) holds. 
Let $A$ be in $\widetilde{H \Psi}_{p}^{m, \nu}$ (resp. $\left.\Psi_{p}^{m, \nu}\left(B_{\sigma}(R)\right)\right)$ and $B$ be in $\widetilde{H \Psi}_{q}^{m^{\prime}, \nu^{\prime}}$ (resp. $\left.\Psi_{q}^{m^{\prime}, \nu^{\prime}}\left(B_{\sigma}(R)\right)\right)$. Denote by a a principal symbol of $A$ modulo $\widetilde{H \Psi}_{p}^{m-1, \nu+M_{0}(3 d+4)}$ (resp. $\Psi_{p}^{m-1, \nu+M_{0}(3 d+4)}\left(B_{\sigma}(R)\right)$ ) and by $b$ a principal symbol of $B$ modulo $\widetilde{H \Psi} \Psi^{m^{\prime}-1, \nu^{\prime}+M_{0}(3 d+4)}$ (resp. $\left.\Psi_{q}^{m^{\prime}-1, \nu^{\prime}+M_{0}(3 d+4)}\left(B_{\sigma}(R)\right)\right)$. Then $a b$ is a principal symbol for $A \circ B$ modulo $\widetilde{H \Psi}_{p+q}^{m+m^{\prime}-1, \nu+M_{0}(3 d+4)}$ when $p>0, q>0$, modulo $\widetilde{H \Psi}_{p+q}^{m+m^{\prime}-1, \nu+\nu^{\prime}+2 M_{0}(2 d+3)}$ if $p$ or $q$ is zero (resp. modulo $\Psi_{\max (p, q)}^{m+m^{\prime}-1, \max \left(\nu, \nu^{\prime}\right)+M_{0}(3 d+4)}\left(B_{\sigma}(R)\right)$ ).

(iv) With the notations of (iii), $a^{\vee}$ (resp. $\bar{a}$ ) is a principal symbols of ${ }^{t} A$ and $A^{*}$ modulo $\widetilde{H \Psi}_{p}^{m-1, \nu+M_{0}(3 d+5)}$ (resp. $\left.\Psi_{p}^{m, \nu+M_{0}(3 d+5)}\left(B_{\sigma}(R)\right)\right)$.

Proof: (i) We consider $\left(\theta_{i}\right)_{i \in I}$ a finite partition of unity on $X$, such that, for each couple $(i, j)$ of $J=\left\{(i, j) \in I \times I ; \operatorname{Supp} \theta_{i} \cap \operatorname{Supp} \theta_{j} \neq \emptyset\right\}$, there is a chart $\kappa_{i j}: V_{i j} \stackrel{\sim}{\rightarrow} \tilde{V}_{i j}$ from the open set $V_{i j}$ of $X$ to the open set $\tilde{V}_{i j}$ of $\mathbb{R}^{d}$ such that $\operatorname{Supp} \theta_{i} \cup \operatorname{Supp} \theta_{j} \subset V_{i j}$. We have seen already in the proof of Lemma 2.2.2, that $\sum_{(i, j) \notin J} \theta_{i} A \theta_{j}$ is in $\widetilde{H \Psi}_{p}^{m-1, \nu+M_{0}}\left(\right.$ resp. $\left.\Psi_{p}^{m-1, \nu+M_{0}}\left(B_{\sigma}(R)\right)\right)$. For each $i$ in $I$, choose $\tilde{\theta}_{i}$ a smooth function such that $\tilde{\theta}_{i} \theta_{i}=\theta_{i}$ and that $\operatorname{Supp} \tilde{\theta}_{i}$ is a compact subset of $V_{i j}$ for any $j$ such that $(i, j) \in J$. We consider

$$
\left(\kappa_{i j}^{-1}\right)^{*} \theta_{i} A \theta_{j}\left(\kappa_{i j}\right)^{*}=\left(\theta_{i} \circ \kappa_{i j}^{-1}\right)\left[\left(\kappa_{i j}^{-1}\right)^{*} \tilde{\theta}_{i} A \tilde{\theta}_{j}\left(\kappa_{i j}\right)^{*}\right]\left(\theta_{j} \circ \kappa_{i j}^{-1}\right) .
$$

The characterization of $\widetilde{H \Psi}_{p}^{m, \nu}$ (resp. $\left.\Psi_{p}^{m, \nu}\left(B_{\sigma}(R)\right)\right)$ in terms of Sobolev spaces given in (1.2.7), (1.2.18), (1.2.19) and Definition 2.1.1 imply that the term between brackets is in $\widetilde{H \Psi}_{p, \text { loc }}^{m, \nu}$ (resp. $\left.\Psi_{p, \text { loc }}^{m, \nu}\left(B_{\sigma}(R)\right)\right)$. By Proposition 2.1.6, we may find a symbol $a_{i j}$ in $\widetilde{H S}_{p, \nu \text { loc }}^{m, \nu}$ (resp. in $\left.S_{p, \text { loc }}^{m, \nu}\left(B_{\sigma}(R)\right)\right)$ such that

$$
\left(\kappa_{i j}^{-1}\right)^{*} \theta_{i} A \theta_{j}\left(\kappa_{i j}\right)^{*}=\left(\theta_{i} \circ \kappa_{i j}^{-1}\right) \operatorname{Op}\left(a_{i j, \chi}\right)\left(\theta_{i} \circ \kappa_{i j}^{-1}\right)
$$

modulo $\widetilde{H \Psi}_{p, \text { loc }}^{m-1, \nu+M_{0}(2 d+3)}$ (resp. $\left.\Psi_{p, \text { loc }}^{m-1, \nu+M_{0}(2 d+3)}\left(B_{\sigma}(R)\right)\right)$. We define a symbol on $C^{\infty}(X)^{p} \times$ $T^{*} X$ (resp. on $\left.B_{\sigma}(R) \times T^{*} X\right)$ by

$$
a=\sum_{(i, j) \in I \times I}\left(\theta_{i} \theta_{j} a_{i j}\right) \circ \tau_{\kappa_{i j}} .
$$

Let $\kappa: V \stackrel{\sim}{\rightarrow} \tilde{V}$ be a local coordinate system, $\theta, \tilde{\theta}, \theta^{\prime}, \tilde{\theta}^{\prime}$ be in $C_{0}^{\infty}(V)$ with $\tilde{\theta} \theta=\theta, \tilde{\theta}^{\prime} \theta^{\prime}=\theta^{\prime}$. Let us show that (2.2.18) holds with the symbol we just defined. We write, modulo $\widetilde{H \Psi}_{p, \text { loc }}^{m,-1, \nu+M_{0}(2 d+3)}$ (resp. $\left.\Psi_{p}^{m-1, \nu+M_{0}(2 d+3)}\left(B_{\sigma}(R)\right)\right)$,

$$
\begin{aligned}
\left(\kappa^{-1}\right)^{*} \theta A \theta^{\prime} \kappa^{*} & =\sum_{(i, j) \in J}\left(\kappa^{-1}\right)^{*} \theta \theta_{i} A \theta_{j} \theta^{\prime} \kappa^{*} \\
& =\sum_{(i, j) \in J}\left(\kappa^{-1}\right)^{*} \theta\left(\kappa_{i j}\right)^{*}\left(\kappa_{i j}^{-1}\right)^{*}\left(\theta_{i} A \theta_{j}\right)\left(\kappa_{i j}\right)^{*}\left(\kappa_{i j}^{-1}\right)^{*} \theta^{\prime} \kappa^{*} \\
& =\sum_{(i, j) \in J}\left(\left(\left.\kappa \circ \kappa_{i j}^{-1}\right|_{\kappa_{i j}\left(V \cap V_{i j}\right)}\right)^{-1}\right)^{*}\left(\theta \theta_{i}\right) \circ \kappa_{i j}^{-1} \mathrm{Op}\left(a_{i j, \chi}\right)\left(\theta^{\prime} \theta_{j}\right) \circ \kappa_{i j}^{-1}\left(\left.\kappa \circ \kappa_{i j}^{-1}\right|_{\kappa_{i j}\left(V \cap V_{i j}\right)}\right)^{*}
\end{aligned}
$$


where the last equality comes from (2.2.19). By formula (2.2.2) of Proposition 2.2.1, the last term may be written, modulo $\widetilde{H \Psi}_{p, \text { loc }}^{m-1, \nu+M_{0}(3 d+4)}$ (resp. $\left.\Psi_{p, \text { loc }}^{m-1, \nu+M_{0}(3 d+4)}\left(B_{\sigma}(R)\right)\right)$ as

$$
\sum_{(i, j) \in J}\left(\tilde{\theta} \circ \kappa^{-1}\right) \operatorname{Op}\left[\left(\theta \theta^{\prime} \theta_{i} \theta_{j} a_{i j}\right) \circ \tau_{\kappa_{i j}} \circ \tau_{\kappa}^{-1}\right]_{\chi_{1}}\left(\tilde{\theta}^{\prime} \circ \kappa^{-1}\right) .
$$

Using (2.2.20) (and that $\theta_{i} \theta_{j}=0$ if $\left.(i, j) \notin J\right)$, we obtain $\left(\tilde{\theta} \circ \kappa^{-1}\right) \operatorname{Op}\left[\left(a \circ \tau_{\kappa}^{-1}\right)_{\chi_{1}}\right]\left(\tilde{\theta}^{\prime} \circ \kappa^{-1}\right)$ i.e. the right hand side of (2.2.18).

(ii) Let $\left(\theta_{i}\right)_{i \in I}$ be a partition of unity on $X$ such that $\operatorname{Supp} \theta_{i}$ is contained in an open set $V_{i}$ so that there is a local chart $\kappa_{i}: V_{i} \stackrel{\sim}{\rightarrow} \tilde{V}_{i} \subset \mathbb{R}^{d}$. Take $\tilde{\theta}_{i}$ in $C_{0}^{\infty}\left(V_{i}\right)$ with $\tilde{\theta}_{i} \theta_{i}=\theta_{i}$ and define $A$ by

$$
A=\sum_{i \in I} \kappa_{i}^{*}\left(\tilde{\theta}_{i} \circ \kappa_{i}^{-1}\right) \operatorname{Op}\left[\left(\theta_{i} a\right) \circ \tau_{\kappa_{i}}^{-1}\right]_{\chi}\left(\tilde{\theta}_{i} \circ \kappa_{i}^{-1}\right)\left(\kappa_{i}^{-1}\right)^{*}
$$

where $\chi \in C_{0}^{\infty}\left(\mathbb{R}^{d}\right), \chi \equiv 1$ close to zero, Supp $\chi$ small enough. By (ii) of Proposition 2.1.6, $A$ is an element of $\widetilde{H \Psi}_{p}^{m, \nu+2 M_{0}(d+1)}$ (resp. $\Psi_{p}^{m, \nu+2 M_{0}(d+1)}\left(B_{\sigma}(R)\right)$ ). Let us check that $a$ satisfies (2.2.18). If $\kappa, \theta, \theta^{\prime}$ are as in the statement of Definition 2.1.5,

$$
\begin{aligned}
& \left(\kappa^{-1}\right)^{*} \theta A \theta^{\prime} \kappa^{*} \\
& =\sum_{i \in I}\left(\tilde{\theta} \circ \kappa^{-1}\right)\left[\left(\kappa_{i} \circ \kappa^{-1}\right)^{*}\left(\theta \tilde{\theta}_{i} \circ \kappa_{i}^{-1}\right) \operatorname{Op}\left[\left(\theta_{i} a\right) \circ \tau_{\kappa_{i}}^{-1}\right]_{\chi}\left(\theta^{\prime} \tilde{\theta}_{i} \circ \kappa_{i}^{-1}\right)\left(\kappa \circ \kappa_{i}^{-1}\right)^{*}\right]\left(\tilde{\theta}^{\prime} \circ \kappa^{-1}\right)
\end{aligned}
$$

if $\tilde{\theta}, \tilde{\theta}^{\prime}$ are in $C_{0}^{\infty}(V)$ with $\theta \tilde{\theta}=\theta, \theta^{\prime} \tilde{\theta}^{\prime}=\theta^{\prime}$. We apply Proposition 2.2.1, to get modulo $\widetilde{H \Psi}_{p, \text { loc }}^{m-1, \nu+M_{0}(3 d+4)}\left(\right.$ resp. $\left.\Psi_{p, \text { loc }}^{m-1, \nu+M_{0}(3 d+4)}\left(B_{\sigma}(R)\right)\right)$ the expression

$$
\sum_{i \in I}\left(\tilde{\theta} \circ \kappa^{-1}\right) \operatorname{Op}\left[\left(\left(\theta \theta^{\prime} \theta_{i} a\right) \circ \tau_{\kappa}^{-1}\right)_{\chi_{1}}\right]\left(\tilde{\theta}^{\prime} \circ \kappa^{-1}\right)=\left(\tilde{\theta} \circ \kappa^{-1}\right) \operatorname{Op}\left[\left(\left(\theta \theta^{\prime} a\right) \circ \tau_{\kappa}^{-1}\right)_{\chi_{1}}\right]\left(\tilde{\theta}^{\prime} \circ \kappa^{-1}\right) .
$$

(iii) We just need to check the property locally. We may always assume that the distribution kernels of $A$ and $B$ are supported in a small neighborhood $W$ of the diagonal. Let $\kappa: V \stackrel{\sim}{\rightarrow} \tilde{V}$ be a chart and $\theta, \theta^{\prime}$ in $C_{0}^{\infty}(V)$. If $W$ is small enough, we may find $\theta_{1}$ in $C_{0}^{\infty}(V)$ with $\theta \theta_{1}=\theta$, $\theta^{\prime} \theta_{1}=\theta^{\prime}$ so that

$$
\left(\kappa^{-1}\right)^{*} \theta A B \theta^{\prime} \kappa^{*}=\left[\left(\kappa^{-1}\right)^{*} \theta A \theta_{1} \kappa^{*}\right]\left[\left(\kappa^{-1}\right)^{*} \theta_{1} B \theta^{\prime} \kappa^{*}\right] .
$$

By Definition 2.2.5, Lemma 1.2.4 and Lemma 1.2.6, the last expression may be written, modulo $\widetilde{H \Psi}_{p+q, \text { loc }}^{m+m^{\prime}-1, \nu+M_{0}(3 d+4)}\left(\right.$ resp. $\left.\Psi_{\max (p, q), \text { loc }}^{m+m^{\prime}-1, \max \left(\nu, \nu^{\prime}\right)+M_{0}(3 d+4)}\left(B_{\sigma}(R)\right)\right)$ as

$$
\left(\tilde{\theta} \circ \kappa^{-1}\right) \operatorname{Op}\left[\left((\theta a) \circ \tau_{\kappa}^{-1}\right)_{\chi}\right]\left(\tilde{\theta}_{1} \circ \kappa^{-1}\right)^{2} \mathrm{Op}\left[\left(\left(\theta^{\prime} b\right) \circ \tau_{\kappa}^{-1}\right)_{\chi}\right]\left(\tilde{\theta}^{\prime} \circ \kappa^{-1}\right)
$$

where $\tilde{\theta}_{1} \theta_{1}=\theta_{1}, \tilde{\theta} \theta=\theta, \tilde{\theta}^{\prime} \theta^{\prime}=\theta^{\prime}$, when $p>0$ and $q>0$. When $p=0$ or $q=0$, the equality is valid modulo $\widetilde{H \Psi}_{p+q \text {,loc }}^{m+m^{\prime}-1, \nu+\nu^{\prime}+M_{0}(3 d+4)}$ (resp. $\left.\Psi_{\max (p, q), \operatorname{loc}}^{m+m^{\prime}-1, \max \left(\nu, \nu^{\prime}\right)+M_{0}(3 d+4)}\left(B_{\sigma}(R)\right)\right)$. By Theorem 2.1.7 (see (2.1.13) and (2.1.17)), we may write (2.2.22) as

$$
\left(\tilde{\theta} \circ \kappa^{-1}\right) \operatorname{Op}\left[\left(\left(\theta \theta^{\prime} a b\right) \circ \tau_{\kappa}^{-1}\right)_{\chi}\right]\left(\tilde{\theta}_{1} \circ \kappa^{-1}\right)
$$


modulo an element of $\widetilde{H \Psi}_{p+q \text {,loc }}^{m+m^{\prime}-1, \nu+\nu^{\prime}+M_{0}(2 d+3)}$ when $p>0, q>0$ and modulo an element of $\widetilde{H \Psi}_{p+q, \text { loc }}^{m+m^{\prime}-1, \nu+\nu^{\prime}+2 M_{0}(2 d+3)}$ if $p=0$ or $q=0$ (resp. modulo $\Psi_{\max (p, q), \operatorname{loc}}^{m+m^{\prime}-1, \max \left(\nu, \nu^{\prime}\right)+M_{0}(2 d+3)}\left(B_{\sigma}(R)\right)$ ). This shows that $a b$ is a principal symbol of the composition.

(iv) With the same notations as above, we need to study ${ }^{t}\left[\left(\kappa^{-1}\right)^{*} \theta A \theta^{\prime} \kappa^{*}\right]$ (where we denote by the same notation transpose of maps from $L^{2}(X, d \mu)$ to $L^{2}(X, d \mu)$, from $L^{2}\left(\mathbb{R}^{d}, d x\right)$ to $L^{2}\left(\mathbb{R}^{d}, d x\right)$, from $L^{2}(X, d \mu)$ to $L^{2}\left(\mathbb{R}^{d}, d x\right)$ and from $L^{2}\left(\mathbb{R}^{d}, d x\right)$ to $L^{2}(X, d \mu)$. Denote by $\alpha$ the density of the measure $d \mu$ relatively to Lebesgue measure in the coordinate patch i.e. $d \mu(y)=\alpha(x) d x$ if $y=\kappa^{-1}(x)$. Then ${ }^{t}\left(\kappa^{*}\right)=\left(\kappa^{-1}\right)^{*}(\alpha \circ \kappa),{ }^{t}\left(\kappa^{-1}\right)^{*}=(\alpha \circ \kappa)^{-1} \kappa^{*}$, so that

$$
\left[\left(\kappa^{-1}\right)^{*} \theta^{t} A \theta^{\prime} \kappa^{*}\right]={ }^{t}\left[\left(\kappa^{-1}\right)^{*} \theta^{\prime}(\alpha \circ \kappa) A(\alpha \circ \kappa)^{-1} \theta \kappa^{*}\right] .
$$

We apply (2.2.18) and (2.1.18) to write this quantity as

$$
\left(\tilde{\theta} \circ \kappa^{-1}\right) \operatorname{Op}\left[\left(\theta \theta^{\prime} a^{\vee}\right)_{\chi}\right]\left(\tilde{\theta}^{\prime} \circ \kappa^{-1}\right)
$$

modulo $\widetilde{H \Psi}_{p, \text { loc }}^{m-1, \nu+M_{0}(3 d+5)}$ (resp. $\Psi_{p \text {,loc }}^{m-1, \nu+M_{0}(3 d+5)}\left(B_{\sigma}(R)\right)$ ). This concludes the proof, the case of the adjoint being similar.

\section{Quasi-linear Birkhoff normal forms method}

\subsection{Description of the method}

Our goal is to prove Theorem 1.2.8. We fix some integer $P \in \mathbb{N}^{*}$ and take $G$ a function as in the statement of the theorem. We fix also $\mathbf{m} \in] 0,+\infty[-\mathcal{N}$, where $\mathcal{N}$ is the zero measure subset of ] $0,+\infty$ [ introduced before the statement of Theorem 1.2.8. Then inequalities (1.2.27) hold.

To prove the theorem, i.e. to show that the solution of (1.2.28) extends to a time interval ] $-T_{\epsilon}, T_{\epsilon}$ [ with $T_{\epsilon} \geq c \epsilon^{-P}$, for all $u_{0}$ in the unit ball of $H^{s}(X)\left(s>s_{0}\right)$ and any small enough $\epsilon$, it is enough to construct a function $\Theta_{s}$, defined on a neighborhood of zero in $H^{s}(X)$, real valued, such that there is $C>0, R>0$ with

$$
C^{-1}\|u\|_{H^{s}}^{2} \leq \Theta_{s}(u) \leq C\|u\|_{H^{s}}^{2} \text { for all } u \text { in } B_{s}(R)
$$

and for any solution $u$ of $u_{t}=i \nabla_{\bar{u}} G(u, \bar{u})$, defined and smooth on $]-T, T[\times X$ for some $T$, the estimate

$$
\Theta_{s}(u(t, \cdot)) \leq \Theta_{s}(u(0, \cdot))+C\left|\int_{0}^{t}\|u(\tau, \cdot)\|_{H^{s}}^{P+2} d \tau\right|+C \epsilon^{P}\left|\int_{0}^{t}\|u(\tau, \cdot)\|_{H^{s}}^{2} d \tau\right|
$$

holds for all $t$ in $]-T, T$ [ such that $u(t, \cdot) \in B_{s}(R)$. Actually, (3.1.1) and (3.1.2) imply an estimate

$$
\|u(t, \cdot)\|_{H^{s}}^{2} \leq C\left[\|u(0, \cdot)\|_{H^{s}}^{2}+\left|\int_{0}^{t}\|u(\tau, \cdot)\|_{H^{s}}^{P+2} d \tau\right|+\epsilon^{P}\left|\int_{0}^{t}\|u(\tau, \cdot)\|_{H^{s}}^{2} d \tau\right|\right]
$$


as long as $u(t, \cdot)$ stays in $B_{s}(R) \cap C^{\infty}$. This shows that for $u(0, \cdot)$ smooth and $\|u(0, \cdot)\|_{H^{s}} \leq \epsilon$ small enough, the solution extends up to a time $T_{\epsilon} \geq c \epsilon^{-P}$ for some small $c>0$. When $u(0, \cdot)$ is only in $H^{s}(X)$, with small enough $H^{s}$-norm, we may always approximate the Cauchy data by $C^{\infty}(X)$ functions, solve the equation for these approximations on $]-T_{\epsilon}, T_{\epsilon}$ [ and pass to the limit to get the solution of (1.2.28) for any $u_{0}$ in the unit ball of $H^{s}(X)$ and any $\epsilon>0$ small enough.

The construction of $\Theta_{s}$ will be made in three steps, given by the following three theorems.

Theorem 3.1.1 There is $\nu \in \mathbb{R}_{+}, s_{0}>0, R>0$ and for any $s>s_{0}$, a canonical transformation $\chi$ defined on $B_{s}(R)$, with $\chi(0)=0, \chi^{\prime}(0)=\mathrm{Id}$, and there are

- Elements $A_{p}$ of $H \Psi_{p}^{1, \nu}, 1 \leq p \leq P-1$, self-adjoint,

- Elements $A_{P}, B_{P}$ of $\Psi_{P}^{1, \nu}\left(B_{s}(R)\right)$, with $A_{P}$ self-adjoint,

- Functions $G_{L, p}$ in $\mathcal{G}_{p}^{1-\infty, \nu+\infty}\left(B_{s}(R)\right), 1 \leq p \leq P-1$,

such that for any $C^{1}$ function $\Theta_{s}^{1}$, defined on $B_{s}(R)$, vanishing at order 2 at the origin, one has, for any $U$ in $B_{s}(R) \cap C^{\infty}$

$$
\left\{\Theta_{s}^{1} \circ \chi, G\right\}(U)=\left\{\Theta_{s}^{1}, G_{L}+G_{\mathrm{H}}+\tilde{G}_{P}\right\} \circ \chi(U)+O\left(\|u\|_{H^{s}}^{P+2}\right), U \rightarrow 0
$$

with

$$
G_{\mathrm{H}}(U)=G_{0}(u)+\sum_{p=1}^{P-1} \int_{X}\left(A_{p}(U) u\right) \bar{u} d \mu
$$

and

$$
\tilde{G}_{P}(U)=\int_{X}\left(A_{P}(U) u\right) \bar{u} d \mu+\operatorname{Re} \int_{X}\left(B_{P}(U) u\right) u d \mu .
$$

Remarks • We write the preceding statement for functions $U=(u, \bar{u})$ in $B_{s}(R) \cap C^{\infty}$ so that the Poisson brackets in (3.1.3) are well defined. Actually, since (3.1.5), (3.1.6) involve operators of order one, $X_{G_{\mathrm{H}}}, X_{\tilde{G}_{P}}$ are in $H^{s-1}(X)$ if $U$ is in $H^{s}$. On the other hand, $d \Theta_{s}^{1}(U)$ is in $\mathcal{L}\left(H^{s}, \mathbb{R}\right)$, so that $d \Theta_{s}^{1}(U) \cdot X_{G_{L}+G_{\mathrm{H}}+\tilde{G}_{P}}$ is not necessarily well defined if $U$ is assumed to be only in $H^{s}$. Of course, at the end of the proof, $\Theta_{s}^{1}$ will be defined in such a way that the right hand side of (3.1.3) will extend even to function $U$ that belong only to $H^{s}$.

- The conclusion of the theorem asserts that we may choose $\chi$ in such a way that the new Hamiltonian in the right hand side of (3.1.3) contains contributions homogeneous of lower degree $0 \leq p \leq P-1$ that are hyperbolic (in the sense of Definition 1.2.7), which form $G_{\mathrm{H}}$, "lower order" contributions giving $G_{L}$, and a term $\tilde{G}_{P}$, which has no special structure, but vanishes at high order when $U$ goes to zero.

The second step in the proof of theorem 1.2.8 will eliminate the elliptic part of $\tilde{G}_{P}$. 
Theorem 3.1.2 Let $\nu \in \mathbb{R}_{+}$be given. There is $s_{0}>0$ such that if $s \geq s_{0}$, there is a local diffeomorphism $\psi$ from a neighborhood of 0 in $H^{s}(X)$ to a neighborhood of zero in $H^{s}(X)$, with $\psi(0)=0$, such that, for any function $\Theta_{s}^{2}$ which is in $\bigoplus_{p=0}^{P-1}\left(H \mathcal{F}_{p, \mathrm{H}}^{2 s, \nu}\left(B_{s}(R)\right)+\mathcal{G}_{p}^{2 s-1, \nu}\left(B_{s}(R)\right)\right)$, for any $U$ in $C^{\infty}(X)$ belonging to a small enough neighborhood of zero in $H^{s}(X)$,

$$
\left\{\Theta_{s}^{2} \circ \psi, G_{L}+G_{\mathrm{H}}+\tilde{G}_{P}\right\}(U)=\left\{\Theta_{s}^{2}, G_{L}+G_{\mathrm{H}}\right\} \circ \psi(U)+O\left(\|U\|_{H^{s}}^{P+2}\right) .
$$

The last step of the proof will be to choose $\Theta_{s}^{2}$ so that the first term in the right hand side of (3.1.7) vanishes essentially at order $P+2$ at zero.

Theorem 3.1.3 There are $\nu \in \mathbb{R}_{+}, s_{0}>0$ and, for any $s \geq s_{0}$, a constant $C_{0}>0$ and, for any $\epsilon \in] 0,1\left[\right.$, any $R>0$, an element $\Theta_{s}^{2}=\Theta_{s}^{2}(\epsilon)$ in $\bigoplus_{p \geq 0} H \overline{\mathcal{F}}_{p, \mathrm{H}}^{2 s, \nu}\left(B_{s}(R)\right)+\mathcal{G}_{p}^{2 s-1, \nu}\left(B_{s}(R)\right)$, with estimates uniform in $\epsilon \in] 0,1[$, satisfying (3.1.1) uniformly in $\epsilon \in] 0,1[$, such that

$$
\left|\left\{\Theta_{s}^{2}, G_{L}+G_{\mathrm{H}}\right\}(U)\right| \leq C_{0}\left[\|U\|_{H^{s}}^{P+2}+\epsilon^{P}\|U\|_{H^{s}}^{2}\right]
$$

for any $U$ in $C^{\infty}(X) \cap B_{s}(R)$.

Proof of theorem 1.2.8: As already seen, we just need to construct a function $\Theta_{s}$ such that (3.1.1) and (3.1.2) hold (actually, $\Theta_{s}$ will be a family of functions, depending on $\epsilon$, and for which (3.1.1), (3.1.2) hold with constants independent of $\epsilon \in] 0,1[$ ). We take $\nu$ large enough so that Theorems 3.1.1 and 3.1.3 hold, and $s_{0}$ large enough so that Theorems 3.1.1, 3.1.2 and 3.1.3 apply. We use Theorem 3.1.1 to determine $\chi, G_{\mathrm{H}}, \tilde{G}_{P}$. Then, Theorem 3.1.3 determines $\Theta_{s}^{2}$ and Theorem 3.1.2 determines $\psi$. We set $\Theta_{s}^{1}=\Theta_{s}^{2} \circ \psi$ and $\Theta_{s}=\Theta_{s}^{1} \circ \chi$. Then, (3.1.3), (3.1.7) and (3.1.8) show that $\left|\left\{\Theta_{s}, G\right\}\right|$ is bounded from above by the right hand side of (3.1.8). Since it follows from equation (1.2.18) that $\frac{d}{d t} \Theta_{s}(u(t, \cdot))=\left\{\Theta_{s}, G\right\}(u(t, \cdot))$, this gives $(3.1 .2)$ by integration. Inequality (3.1.1) holds by construction. This concludes the proof of Theorem 1.2.8 from the three results in this subsection.

The proofs of Theorems 3.1.1 to 3.1.3 will depend on the study of Poisson brackets between functions of the classes of Definition 1.2.7. We shall study such Poisson brackets in the following subsection.

\subsection{Computation of Poisson brackets}

Let $U \rightarrow A(U)$ be a $C^{1}$ function defined on an open set $\Omega$ of $H^{\sigma}(X)$, with $\sigma$ large enough, with values in $\mathcal{L}\left(H^{s}, H^{s-m}\right)$ for some $m$ and any $s$ with $s \geq \sigma$ and $m \leq 2 s$. Consider the functions

$$
\begin{aligned}
H_{1}^{A}(W, U) & =\int_{X}(A(W) u) u d \mu \\
H_{0}^{A}(W, U) & =\int_{X}(A(W) u) \bar{u} d \mu \\
H_{-1}^{A}(W, U) & =\int_{X}(A(W) \bar{u}) \bar{u} d \mu
\end{aligned}
$$


defined for $W \in \Omega, U=(u, \bar{u}) \in H^{s}(X)$. If $U$ is in $\Omega \cap H^{s}(X)$, set $F_{a}^{A}(U)=H_{a}^{A}(U, U)$, $a=-1,0,1$. Let $B$ be another $C^{1}$ map from $\Omega$ to $\mathcal{L}\left(H^{s}, H^{s-m^{\prime}}\right)$ for some $m^{\prime}$ and any $s \geq \sigma$, $m^{\prime} \leq 2 s$. Assume moreover $m+m^{\prime} \leq 2 s$. By formulas (1.1.17), (1.1.16)

$$
\begin{aligned}
\left\{F_{a}^{A}, F_{b}^{B}\right\}= & \left.\left\{H_{a}^{A}(W, \cdot), H_{b}^{B}(W, \cdot)\right\}\right|_{W=U}+i\left(d_{W} H_{a}^{A}\right)(U, U)^{t} J\left(\nabla_{U} H_{b}^{B}\right)(U, U) \\
& -i\left(d_{W} H_{b}^{B}\right)(U, U)^{t} J\left(\nabla_{U} H_{a}^{A}\right)(U, U)+\left.\left\{H_{a}^{A}(\cdot, U), H_{b}^{B}(\cdot, U)\right\}\right|_{W=U}
\end{aligned}
$$

where the first (resp. the last) term in the right hand side is the Poisson bracket of the functions $U \rightarrow H_{a}^{A}(W, U), U \rightarrow H_{b}^{B}(W, U)$ (resp. $\left.W \rightarrow H_{a}^{A}(W, U), W \rightarrow H_{b}^{B}(W, U)\right)$ at fixed $W$ (resp. $U)$. Let us compute explicitly the first Poisson bracket. According to formula (1.1.17)

$$
\begin{aligned}
\left\{H_{1}^{A}(W, \cdot), H_{0}^{B}(W, \cdot)\right\} & =i \int_{X}\left(\left(A(W)+{ }^{t} A(W)\right) u\right)(B(W) u) d \mu \\
\left\{H_{1}^{A}(W, \cdot), H_{-1}^{B}(W, \cdot)\right\} & =i \int_{X}\left(\left(A(W)+{ }^{t} A(W)\right) u\right)\left(\left(B(W)+{ }^{t} B(W)\right) \bar{u}\right) d \mu \\
\left\{H_{0}^{A}(W, \cdot), H_{-1}^{B}(W, \cdot)\right\} & =i \int_{X}\left({ }^{t} A(W) \bar{u}\right)\left(\left(B(W)+{ }^{t} B(W)\right) \bar{u}\right) d \mu
\end{aligned}
$$

and

$$
\begin{aligned}
& \left\{H_{1}^{A}(W, \cdot), H_{1}^{B}(W, \cdot)\right\}=0,\left\{H_{-1}^{A}(W, \cdot), H_{-1}^{B}(W, \cdot)\right\}=0 \\
& \left\{H_{0}^{A}(W, \cdot), H_{0}^{B}(W, \cdot)\right\}=i \int_{X}([A, B](W) u) \bar{u} d \mu .
\end{aligned}
$$

Consequently, the first term in the right hand side of (3.2.2) may be written as $H_{c}^{C}(U, U)$ for some $C$ that may be computed from compositions of $A, B,{ }^{t} A,{ }^{t} B$ or from a commutator $[A, B]$. On the other hand, the second term in the right hand side of (3.2.2), has the following structure for $a=1,0,-1$

$$
\begin{aligned}
& i \int_{X}\left[d_{W} A(W) \cdot\left({ }^{t} J \nabla_{U} H_{b}^{B}\right) u\right] u d \mu \\
& i \int_{X}\left[d_{W} A(W) \cdot\left({ }^{t} J \nabla_{U} H_{b}^{B}\right) u\right] \bar{u} d \mu \\
& i \int_{X}\left[d_{W} A(W) \cdot\left({ }^{t} J \nabla_{U} H_{b}^{B}\right) \bar{u}\right] \bar{u} d \mu .
\end{aligned}
$$

By definition of $H_{b}^{B}$,

$$
\begin{gathered}
\nabla_{U} H_{1}^{B}(W, U)=\left[\begin{array}{c}
\left(B(W)+{ }^{t} B(W)\right) u \\
0
\end{array}\right], \nabla_{U} H_{0}^{B}(W, U)=\left[\begin{array}{c}
{ }^{t} B(W) \bar{u} \\
B(W) u
\end{array}\right] \\
\nabla_{U} H_{-1}^{B}(W, U)=\left[\begin{array}{c}
0 \\
\left(B(W)+{ }^{t} B(W)\right) \bar{u}
\end{array}\right]
\end{gathered}
$$

so that (3.2.5) will be computed from quantities of the form

$$
d_{w} A(W) \cdot(C(W) u), d_{w} A(W) \cdot(C(W) \bar{u}), d_{\bar{w}} A(W) \cdot(C(W) u), d_{\bar{w}} A(W) \cdot(C(W) \bar{u})
$$

where $C(W)$ will be an operator of the same form as $A(W), B(W)$.

We introduce an auxiliary class of multi-linear operators. 
Definition 3.2.1 Let $m \in \mathbb{R}, \nu \in \mathbb{R}_{+}, p \in \mathbb{N}^{*}$. We denote by $\widetilde{H \mathcal{M}}_{p}^{m, \nu}$ the space of $p$-linear maps $\left(u_{1}, \ldots, u_{p}\right) \rightarrow M\left(u_{1}, \ldots, u_{p}\right)$, defined on $C^{\infty}(X)^{p}$, with values in the space of linear maps from $C^{\infty}(X)$ to $\mathcal{D}^{\prime}(X)$, that may be written as a sum $\sum_{i=1}^{p} M_{i}\left(u_{1}, \ldots, u_{p}\right)$, where $M_{i}$ satisfies the following estimates: for any $N_{1}, N_{2}$ in $\mathbb{N}$, there is $C>0$ and for any $n_{0}, \ldots, n_{p+1}$ in $\mathbb{N}^{*}$

$$
\begin{aligned}
\left\|\Pi_{n_{0}} M_{i}\left(\Pi_{n_{1}} u_{1}, \ldots, \Pi_{n_{p}} u_{p}\right) \Pi_{n_{p+1}}\right\|_{\mathcal{L}\left(L^{2}\right)} \leq C\left[\min \left(\frac{n_{i}}{n_{p+1}}, \frac{n_{p+1}}{n_{i}}\right)\right]^{N_{1}} \max \left(n_{0}, \ldots, \widehat{n_{i}}, \ldots, n_{p}\right)^{\nu(p)+\nu} \\
\times\left(n_{i}+n_{p+1}\right)^{m}\left(1+\frac{\max \left(n_{0}, \ldots, \hat{n}_{i}, \ldots, n_{p}\right)}{n_{i}+n_{p+1}}\right)^{-N_{2}} \prod_{1}^{p}\left\|\Pi_{n_{\ell}} u_{\ell}\right\|_{L^{2}} .
\end{aligned}
$$

We shall denote by $H_{\mathcal{M}}^{m, \nu}$ the space of operator valued maps $U \rightarrow M(U)$ that may be written

$$
M(U)=\sum_{\ell=0}^{p} M_{\ell}(\underbrace{u, \ldots, u}_{\ell}, \underbrace{\bar{u}, \ldots, \bar{u}}_{p-\ell})
$$

with $M_{\ell}$ in $\widetilde{H \mathcal{M}}_{p}^{m, \nu}$.

Lemma 3.2.2 Let $A$ be an element of $\mathcal{P}_{p}^{m, \nu}$ and set for $j \in \mathbb{N}$

$$
H^{A, \leq j}(W, U)=\int_{X}\left(A(W) S_{j} u\right) u d \mu \text { or } \int_{X}\left(A(W) S_{j} u\right) \bar{u} d \mu \text { or } \int_{X}\left(A(W) S_{j} \bar{u}\right) \bar{u} d \mu .
$$

Then $\left.\nabla_{w} H^{A, \leq j}(W, U)\right|_{W=U}$ and $\left.\nabla_{\bar{w}} H^{A, \leq j}(W, U)\right|_{W=U}$ may be written as a linear combination of $M_{\alpha}(U) S_{j} u, M_{\alpha}(U) S_{j} \bar{u}$ for elements $M_{\alpha}$ of $H \mathcal{M}_{p}^{m, \nu}$.

Proof: We consider for instance the first expression of $H^{A, \leq j}$. We decompose $A(W)=$ $\sum_{\ell=0}^{p} A_{\ell}(\underbrace{w, \ldots, w}_{\ell}, \bar{w}, \ldots, \bar{w})$ with $A_{\ell}$ in $\widetilde{\mathcal{P}}_{p}^{m, \nu}$. Then $d_{w} H^{A, \leq j}(W, U) \cdot h$ may be written as a sum

$$
\sum_{\ell=0}^{p} \sum_{i=1}^{\ell} \int_{X}\left(A_{\ell}(w, \ldots, \underbrace{h}_{i}, \ldots, w, \bar{w}, \ldots, \bar{w}) S_{j} u\right) u d \mu .
$$

Consequently, $\left.\nabla_{w} H^{A, \leq j}(W, U)\right|_{W=U}$ may be written as a sum

$$
\sum_{\ell=0}^{p} \sum_{i=1}^{\ell} M_{\ell i}(\underbrace{u, \ldots, u}_{\ell}, \bar{u}, \ldots, \bar{u}) S_{j} u
$$

with

$$
\int_{X}\left(M_{\ell i}\left(u_{1}, \ldots, u_{p}\right) u_{p+1}\right) h d \mu=\int_{X}\left(A_{\ell}\left(u_{1}, \ldots, u_{i-1}, h, u_{i+1}, \ldots, u_{p}\right) u_{p+1}\right) u_{i} d \mu .
$$

The fact that $\left\|\Pi_{n_{0}} M_{\ell i}\left(\Pi_{n_{1}} u_{1}, \ldots, \Pi_{n_{p}} u_{p}\right) \Pi_{n_{p+1}}\right\|_{\mathcal{L}\left(L^{2}\right)}$ is bounded by the right hand side of (3.2.8) follows from (1.2.5).

The goal of this subsection is to prove the following: 
Proposition 3.2.3 Let $m, m^{\prime}$ in $\mathbb{R}, \nu, \nu^{\prime}$ in $\mathbb{R}_{+}, p, q$ in $\mathbb{N}, \sigma \in \mathbb{R}$. Assume

$$
\nu(p)+\nu(q)+\max \left(\nu, \nu^{\prime}\right)+M_{0}(3 d+4) \leq \nu(p+q) \text { if } p>0, q>0
$$

and that $\sigma$ is large enough so that condition (1.2.23) holds for all the spaces of multi-linear forms below. Then, with the notations introduced in definition 1.2.7, one has the following inclusions

$$
\begin{gathered}
\left\{H \mathcal{F}_{p, \mathrm{E}}^{m, \nu}\left(B_{\sigma}(R)\right), H \mathcal{F}_{q, \mathrm{E}}^{m^{\prime}, \nu^{\prime}}\left(B_{\sigma}(R)\right)\right\} \cup\left\{H \mathcal{F}_{p, \mathrm{H}}^{m, \nu}\left(B_{\sigma}(R)\right), H \mathcal{F}_{q, \mathrm{E}}^{m^{\prime}, \nu^{\prime}}\left(B_{\sigma}(R)\right)\right\} \\
\cup\left\{H \mathcal{F}_{p, \mathrm{H}}^{m, \nu}\left(B_{\sigma}(R)\right), H \mathcal{F}_{q, \mathrm{H}}^{m^{\prime}, \nu^{\prime}}\left(B_{\sigma}(R)\right)\right\} \\
\subset H \mathcal{F}_{p+q}^{m+m^{\prime}, \min \left(\nu, \nu^{\prime}\right)}\left(B_{\sigma}(R)\right)+H \mathcal{F}_{p+q}^{m, m_{+}^{\prime}+\min \left(\nu, \nu^{\prime}\right)}\left(B_{\sigma}(R)\right)+H \mathcal{F}_{p+q}^{m^{\prime}, m_{+}+\min \left(\nu, \nu^{\prime}\right)}\left(B_{\sigma}(R)\right)
\end{gathered}
$$

if $p>0$ and $q>0$. If $p$ or $q$ is zero, one gets the same inclusion with $\min \left(\nu, \nu^{\prime}\right)$ replaced by $\nu+\nu^{\prime}$;

$$
\begin{aligned}
\left\{H \mathcal{F}_{p, \mathrm{H}}^{m, \nu}\left(B_{\sigma}(R)\right), H \mathcal{F}_{q, \mathrm{H}}^{m^{\prime}, \nu^{\prime}}\right. & \left.\left(B_{\sigma}(R)\right)\right\} \subset H \mathcal{F}_{p+q, \mathrm{H}}^{m+m^{\prime}-1, \min \left(\nu, \nu^{\prime}\right)+M_{0}(3 d+4)}\left(B_{\sigma}(R)\right) \\
& +H \mathcal{F}_{p+q, \mathrm{H}}^{m, m_{+}^{\prime}+\min \left(\nu, \nu^{\prime}\right)}\left(B_{\sigma}(R)\right)+H \mathcal{F}_{p+q, \mathrm{H}}^{m^{\prime}, m_{+}+\min \left(\nu, \nu^{\prime}\right)}\left(B_{\sigma}(R)\right)
\end{aligned}
$$

if $p>0$ and $q>0$. If $p$ or $q$ is zero, one gets the same inclusion with $\min \left(\nu, \nu^{\prime}\right)$ replaced by $\nu+\nu^{\prime}$ and $M_{0}(3 d+4)$ replaced by $2 M_{0}(2 d+3)$. Moreover, one has also the inclusions

$$
\begin{aligned}
\left\{\mathcal{G}_{p}^{m, \nu}\right. & \left.\left(B_{\sigma}(R)\right), \mathcal{G}_{q}^{m^{\prime}, \nu^{\prime}}\left(B_{\sigma}(R)\right)\right\} \\
& \subset \mathcal{G}_{p+q}^{m+m^{\prime}, \min \left(\nu, \nu^{\prime}\right)}\left(B_{\sigma}(R)\right)+\mathcal{G}_{p+q}^{m, m_{+}^{\prime}+\min \left(\nu, \nu^{\prime}\right)}\left(B_{\sigma}(R)\right)+\mathcal{G}_{p+q}^{m^{\prime}, m_{+}+\min \left(\nu, \nu^{\prime}\right)}\left(B_{\sigma}(R)\right)
\end{aligned}
$$

if $p>0$ and $q>0$. If $p$ or $q$ is zero, one gets the same inclusion with $\min \left(\nu, \nu^{\prime}\right)$ replaced by $\nu+\nu^{\prime}$. One has also

$$
\begin{aligned}
& \left\{H_{\left.\mathcal{F}_{p, \mathrm{H}}^{m, \nu}\left(B_{\sigma}(R)\right), \mathcal{G}_{q}^{m^{\prime}, \nu^{\prime}}\left(B_{\sigma}(R)\right)\right\}}\right. \\
\subset & H \mathcal{F}_{p+q, \mathrm{H}}^{m, m_{+}^{\prime}+\min \left(\nu, \nu^{\prime}\right)}\left(B_{\sigma}(R)\right)+\mathcal{G}_{p+q}^{m+m^{\prime}, \min \left(\nu, \nu^{\prime}\right)+2 M_{0}}\left(B_{\sigma}(R)\right)+\mathcal{G}_{p+q}^{m^{\prime}, m_{+}+\min \left(\nu, \nu^{\prime}\right)}\left(B_{\sigma}(R)\right)
\end{aligned}
$$

if $p>0$ and $q>0$. If $p$ or $q$ is zero, one gets the same inclusion with $\min \left(\nu, \nu^{\prime}\right)$ replaced by $\nu+\nu^{\prime}$ (resp. $\left.\nu+\nu^{\prime}+2 M_{0}\right)$ in the first (resp. last) term in the right hand side.

Finally, one has the inclusion

(3.2.15) $\left\{\operatorname{HF}_{p}^{m, \nu}\left(B_{\sigma}(R)\right), \mathcal{G}_{q}^{m^{\prime}, \nu^{\prime}}\left(B_{\sigma}(R)\right)\right\}$

$$
\subset \mathcal{G}_{p+q}^{m+m^{\prime}, \min \left(\nu, \nu^{\prime}\right)+2 M_{0}}\left(B_{\sigma}(R)\right)+\mathcal{G}_{p+q}^{m, m_{+}^{\prime}+\min \left(\nu, \nu^{\prime}\right)+2 M_{0}}\left(B_{\sigma}(R)\right)+\mathcal{G}_{p+q}^{m^{\prime}, m_{+}+\min \left(\nu, \nu^{\prime}\right)+2 M_{0}}\left(B_{\sigma}(R)\right)
$$

if $p>0$ and $q>0$. If $p$ or $q$ is zero, one gets the same inclusion with $\min \left(\nu, \nu^{\prime}\right)$ replaced by $\nu+\nu^{\prime}$.

The main step to prove the proposition will be to study expressions of type (3.2.7) where $A$ will be an element of $H \Psi_{p}^{m, \nu}$. We consider the different possible situations in the following lemma. 
Lemma 3.2.4 Assume, for some $a \geq 0$,

$$
\nu(p)+\nu(q)+\max \left(\nu, \nu^{\prime}\right)+a \leq \nu(p+q) \text { if } p>0 \text { and } q>0 .
$$

Let $A$ be an element of $\widetilde{H \Psi}_{p}^{m, \nu}$ (resp. $\left.\widetilde{\mathcal{P}}_{p}^{m, \nu}\right)$ and $B$ be an element of $\widetilde{\mathcal{P}}_{q}^{m^{\prime}, \nu^{\prime}+a}$. Define

$$
C\left(u_{1}, \ldots, u_{p+q}\right)=A\left(B\left(u_{1}, \ldots, u_{q}\right) u_{q+1}, u_{q+2}, \ldots, u_{p+q}\right) .
$$

Then $C$ is in $\widetilde{H \Psi}_{p+q}^{m, \min \left(\nu, \nu^{\prime}\right)+m_{+}^{\prime}}$ if $p>0, q>0$ and in $\widetilde{H \Psi}_{p+q}^{m, \nu+\nu^{\prime}+m_{+}^{\prime}+a}$ if $p=0$ or $q=0$. (resp. in $\widetilde{\mathcal{P}}_{p+q}^{m, \min \left(\nu, \nu^{\prime}\right)+m_{+}^{\prime}}$ if $p>0, q>0$ and in $\widetilde{\mathcal{P}}_{p+q}^{m, \nu+\nu^{\prime}+m_{+}^{\prime}+a}$ if $p=0$ or $\left.q=0\right)$.

Proof: Let $P_{1}, \ldots, P_{k}$ be differential operators as in Definition 1.2.1. When $A$ is in $\widetilde{H \Psi}_{p}^{m, \nu}$, denote $\tilde{A}=\operatorname{Ad}_{P_{1}} \cdots \operatorname{Ad}_{P_{k}} A$ and $\tilde{C}=\operatorname{Ad}_{P_{1}} \cdots \operatorname{Ad}_{P_{k}} C$. Compute

$$
\begin{aligned}
& \Delta_{j} \tilde{C}\left(\Pi_{n_{1}} u_{1}, \ldots, \Pi_{n_{p+q}} u_{p+q}\right) \Delta_{j^{\prime}} \\
& \quad=\sum_{n} \Delta_{j} \tilde{A}\left(\Pi_{n} B\left(\Pi_{n_{1}} u_{1}, \ldots, \Pi_{n_{q}} u_{q}\right) \Pi_{n_{q+1}} u_{q+1}, \Pi_{n_{q+2}} u_{q+2}, \ldots, \Pi_{n_{p+q}} u_{p+q}\right) \Delta_{j^{\prime}}
\end{aligned}
$$

By symmetry, we may assume $n_{1} \geq \cdots \geq n_{q}, n_{q+2} \geq \cdots \geq n_{p+q}$. By (1.2.8) and (1.2.5), the $\mathcal{L}\left(L^{2}\right)$-norm of the general term of the sum is bounded from above by a constant times $\prod_{1}^{p+q}\left\|\Pi_{n_{\ell}} u_{\ell}\right\|_{L^{2}}$ times

$$
\begin{aligned}
& 2^{-\left|j-j^{\prime}\right| N_{1}^{\prime}}\left(n+n_{q+2}\right)^{\nu(p)+\nu+M_{0} k} 2^{j \tilde{m}}\left(1+2^{-j}\left(n+n_{q+2}\right)\right)^{-N_{2}^{\prime}} \\
& \times\left[\min \left(\frac{n}{n_{q+1}}, \frac{n_{q+1}}{n}\right)\right]^{N_{1}^{\prime \prime}} n^{m^{\prime}} n_{1}^{\nu(q)+\nu^{\prime}+a}\left(1+\frac{n_{1}}{n}\right)^{-N_{2}^{\prime \prime}}\left\langle n-n_{q+1}\right\rangle^{-2}
\end{aligned}
$$

where $\tilde{m}=m+\sum_{1}^{k} d_{\ell}-k$. We write

$$
\begin{aligned}
& \left(1+\frac{n+n_{q+2}}{2^{j}}\right)\left(1+\frac{n_{1}}{n}\right) \geq 1+\frac{n+n_{q+2}+n_{1}}{2^{j}} \\
& \geq\left(1+\frac{n_{q+1}+n_{q+2}+n_{1}}{2^{j}}\right) \min \left(\frac{n}{n_{q+1}}, \frac{n_{q+1}}{n}\right),
\end{aligned}
$$

so that (3.2.19) is bounded from above by

$$
\begin{aligned}
2^{j \tilde{m}} 2^{-\left|j-j^{\prime}\right| N_{1}^{\prime}}\left(n_{q+1}+n_{q+2}\right. & \left.+n_{1}\right)^{\nu(p)+\nu(q)+\nu+\nu^{\prime}+a+m_{+}^{\prime}+M_{0} k}\left(1+\frac{n_{q+1}+n_{q+2}+n_{1}}{2^{j}}\right)^{-N_{2}^{\prime}} \\
& \times\left\langle n-n_{q+1}\right\rangle^{-2}\left[\min \left(\frac{n}{n_{q+1}}, \frac{n_{q+1}}{n}\right)\right]^{N_{1}^{\prime \prime}-N_{2}^{\prime}-\nu(p)-\nu-m_{+}^{\prime}-M_{0} k}
\end{aligned}
$$

if $N_{1}^{\prime \prime}, N_{2}^{\prime \prime}$ are large enough relatively to $N_{2}^{\prime}, \nu, m_{+}^{\prime}, k$. If we sum this in $n$, we get estimate (1.2.8) of an element of $\widetilde{H \Psi}_{p+q}^{m, \min \left(\nu, \nu^{\prime}\right)+m_{+}^{\prime}}$ when $p>0, q>0$ and (3.2.16) holds. If $p=0$ or $q=0$, we get an element of $\widetilde{H \Psi}_{p+q}^{m, \nu+\nu^{\prime}+m_{+}^{\prime}+a}$. When $A$ is in $\widetilde{\mathcal{P}}_{p}^{m, \nu}$, we bound

$$
\left\|\Pi_{n_{0}} C\left(\Pi_{n_{1}} u_{1}, \ldots, \Pi_{n_{p+q}} u_{p+q}\right) \Pi_{n_{p+q+1}}\right\|_{\mathcal{L}\left(L^{2}\right)}
$$


using (1.2.5), by the product of a constant times $\prod_{1}^{p+q}\left\|\Pi_{n_{\ell}} u_{\ell}\right\|_{L^{2}}$ times

$$
\begin{aligned}
\left\langle n_{0}-n_{p+q+1}\right\rangle^{-2}[\min & \left.\left(\frac{n_{0}}{n_{p+q+1}}, \frac{n_{p+q+1}}{n_{0}}\right)\right]^{N_{1}^{\prime}}\left(n+n_{q+2}\right)^{\nu+\nu(p)} n_{0}^{m}\left(1+\frac{n+n_{q+2}}{n_{0}}\right)^{-N_{2}^{\prime}} \\
& \times\left\langle n-n_{q+1}\right\rangle^{-2}\left[\min \left(\frac{n}{n_{q+1}}, \frac{n_{q+1}}{n}\right)\right]^{N_{1}^{\prime \prime}} n^{m^{\prime}} n_{1}^{\nu(q)+\nu^{\prime}}\left(1+\frac{n_{1}}{n}\right)^{-N_{2}^{\prime \prime}}
\end{aligned}
$$

The rest of the computation is similar as above.

Lemma 3.2.5 Let $p, q \in \mathbb{N}^{*}$. Assume for some $a \geq 0$,

$$
\nu(p)+\nu(q)+\max \left(\nu, \nu^{\prime}\right)+a+1 \leq \nu(p+q) .
$$

Let $A$ be in $\widetilde{H \Psi}_{p}^{m, \nu}$ (resp. $\widetilde{\mathcal{P}}_{p}^{m, \nu}$ ) and $M=\sum_{i=1}^{q} M_{i}$ be in $\widetilde{H \mathcal{M}}_{q}^{m^{\prime}, \nu^{\prime}+a}$. Consider

$$
C\left(u_{1}, \ldots, u_{p+q}\right)=\sum_{j^{\prime \prime}=0}^{+\infty} \sum_{i=1}^{q} A\left(M_{i}\left(u_{1}, \ldots, u_{q}\right) S_{j^{\prime \prime}} u_{q+1}, u_{q+2}, \ldots, u_{p+q}\right) \Delta_{j^{\prime \prime}}
$$

Then $C$ is in $\widetilde{H \Psi}_{p+q}^{m, \min \left(\nu, \nu^{\prime}\right)+m_{+}^{\prime}}$ (resp. $\widetilde{\mathcal{P}}_{p+q}^{m, \min \left(\nu, \nu^{\prime}\right)+m_{+}^{\prime}}$ ).

Proof: We consider the contribution to (3.2.24) of the term corresponding to $i=1$. We write $M$ instead of $M_{1}$. Let $P_{1}, \ldots, P_{k}$ be differential operators on $X$ of orders $d_{1}, \ldots, d_{k}$. We may express $\operatorname{Ad}_{P_{1}} \cdots \operatorname{Ad}_{P_{k}} C$ from

$$
\sum_{j^{\prime \prime}} \operatorname{Ad}_{\mathcal{P}^{\prime}} A\left(M\left(u_{1}, \ldots, u_{q}\right) S_{j^{\prime \prime}} u_{q+1}, u_{q+2}, \ldots, u_{p+q}\right) \operatorname{Ad}_{\mathcal{P}^{\prime \prime}} \Delta_{j^{\prime \prime}}
$$

where $\mathcal{P}=\mathcal{P}^{\prime} \cup \mathcal{P}^{\prime \prime}$ is any partition of $\mathcal{P}=\left(P_{1}, \ldots, P_{k}\right)$. Denote by $k^{\prime}$ (resp. $\left.k^{\prime \prime}\right)$ the cardinal of $\mathcal{P}^{\prime}$ (resp. $\left.\mathcal{P}^{\prime \prime}\right)$ and set $d^{\prime}=\sum_{P_{\ell} \in \mathcal{P}^{\prime}} d_{\ell}, d^{\prime \prime}=\sum_{P_{\ell} \in \mathcal{P}^{\prime \prime}} d_{\ell}$. Then $\left\|\Delta_{j} \operatorname{Ad}_{P_{1}} \cdots \operatorname{Ad}_{P_{k}} C\left(\Pi_{n^{\prime}} \mathcal{U}\right) \Delta_{j^{\prime}}\right\|_{\mathcal{L}\left(L^{2}\right)}$ will be bounded from above by the sum for $\mathcal{P}=\mathcal{P}^{\prime} \cup \mathcal{P}^{\prime \prime}$ of

$$
\begin{aligned}
& \sum_{n} \sum_{j^{\prime \prime}} \sum_{\tilde{j}^{\prime \prime}} \| \Delta_{j} \operatorname{Ad}_{\mathcal{P}^{\prime}} A\left(\Pi_{n} M\left(\Pi_{n_{1}} u_{1}, \ldots, \Pi_{n_{q}} u_{q}\right) S_{j^{\prime \prime}} \Pi_{n_{q+1}} u_{q+1},\right. \\
& \left.\Pi_{n_{q+2}} u_{q+2}, \ldots, \Pi_{n_{p+q}} u_{p+q}\right) \Delta_{\tilde{\jmath}^{\prime \prime}}\left\|_{\mathcal{L}\left(L^{2}\right)}\right\| \tilde{\Delta}_{\tilde{\jmath}^{\prime \prime}}\left(\operatorname{Ad}_{\mathcal{P}^{\prime \prime}} \Delta_{j^{\prime \prime}}\right) \Delta_{j^{\prime}} \|_{\mathcal{L}\left(L^{2}\right)}
\end{aligned}
$$

where $\tilde{\Delta}_{\tilde{j}^{\prime \prime}}$ is a cut-off such that $\tilde{\Delta}_{\tilde{j}^{\prime \prime}} \Delta_{\tilde{j}^{\prime \prime}}=\Delta_{\tilde{j}^{\prime \prime}}$. By symmetry, we may assume that $n_{2} \geq n_{3} \geq$ $\cdots \geq n_{q}, n_{q+2} \geq \cdots \geq n_{p+q}$. Moreover, in the sum, $n_{q+1} \leq C 2^{j^{\prime \prime}}$ for some $C>0$. By (1.2.8) and (3.2.8) with $i=1$, the first factor in the general term of (3.2.25) is bounded from above by the product of $\prod_{1}^{p+q}\left\|\Pi_{n_{\ell}} u_{\ell}\right\|_{L^{2}}$ and

$$
\begin{aligned}
& 2^{-\left|j-\tilde{\jmath}^{\prime \prime}\right| N_{1}^{\prime}}\left(n+n_{q+2}\right)^{\nu(p)+\nu+M_{0} k^{\prime}} 2^{j\left(m+d^{\prime}-k^{\prime}\right)}\left(1+2^{-j}\left(n+n_{q+2}\right)\right)^{-N_{2}^{\prime}} \\
\times & \left(\min \left(\frac{n_{1}}{n_{q+1}}, \frac{n_{q+1}}{n_{1}}\right)\right)^{N_{1}^{\prime \prime}}\left(n+n_{2}\right)^{\nu(q)+\nu^{\prime}}\left(n_{1}+n_{q+1}\right)^{m^{\prime}}\left(1+\frac{n+n_{2}}{n_{1}+n_{q+1}}\right)^{-N_{2}^{\prime \prime}} \mathbb{1}_{n_{q+1} \leq C 2^{j^{\prime \prime}}} .
\end{aligned}
$$


We notice that

$$
\begin{aligned}
\left(1+2^{-j}\left(n+n_{q+2}\right)\right)\left(1+\frac{n+n_{2}}{n_{1}+n_{q+2}}\right) \max \left[\frac{n_{1}}{n_{q+1}}, \frac{n_{q+1}}{n_{1}}\right] 2^{\left|j-j^{\prime \prime}\right|} \\
\geq c\left(1+2^{-j}\left(n_{q+2}+n_{q+1}+n_{2}+n_{1}\right)\right)
\end{aligned}
$$

since $n_{q+1} \leq C 2^{j^{\prime \prime}}$. Moreover, for $\ell=2$ or $\ell=q+2$

$$
\left(n+n_{\ell}\right)\left(1+\frac{n+n_{2}}{n_{1}+n_{q+2}}\right)^{-1} \leq C\left(n_{q+2}+n_{q+1}+n_{2}+n_{1}\right) .
$$

If $N_{1}^{\prime \prime}, N_{2}^{\prime \prime}$ are large enough, we bound the sum in $n$ of (3.2.26) by

$$
\begin{aligned}
C 2^{-N_{1}^{\prime}\left|j-\tilde{j}^{\prime \prime}\right|} 2^{j\left(m+d^{\prime}-k^{\prime}\right)}\left(n_{1}+\cdots+n_{p+q}\right)^{\nu(p)} & +\nu(q)+\nu+\nu^{\prime}+1+m_{+}^{\prime}+M_{0} k^{\prime} \\
& \times\left(1+2^{-j}\left(n_{1}+\cdots+n_{p+q}\right)\right)^{-N_{2}^{\prime}} 2^{N_{2}^{\prime}\left|j-j^{\prime \prime}\right|} .
\end{aligned}
$$

We use Proposition A.1 of the appendix to bound the last factor in (3.2.25) by the expression $C_{N} 2^{-N\left[\left|j^{\prime \prime}-j^{\prime \prime}\right|+\left|j^{\prime \prime}-j^{\prime}\right|\right]} 2^{j\left(d^{\prime \prime}-k^{\prime \prime}\right)}$. If we take $N \gg N_{1}^{\prime} \gg N_{2}^{\prime} \gg N_{1}, N_{2}$ and use (3.2.23), we bound $(3.2 .25)$ by

$$
\begin{aligned}
C 2^{-N_{1}\left|j-j^{\prime}\right|} 2^{j\left(m+\sum d_{\ell}-k\right)}\left(n_{1}+\cdots+\right. & \left.n_{p+q}\right)^{\nu(p+q)+\min \left(\nu, \nu^{\prime}\right)+m_{+}^{\prime}+M_{0} k} \\
& \times\left(1+2^{-j}\left(n_{1}+\cdots+n_{p+q}\right)\right)^{-N_{2}} \prod_{1}^{p+q}\left\|\Pi_{n_{\ell}} u_{\ell}\right\|_{L^{2}} .
\end{aligned}
$$

This is the wanted estimate.

The case when $A$ is in $\widetilde{\mathcal{P}}_{p}^{m, \nu}$ is treated in the same way.

Proof of Proposition 3.2.3: Let $A$ (resp. B) be an element of $H \Psi_{p}^{m, \nu}$ or $\mathcal{P}_{p}^{m, \nu}$ (resp. $H \Psi_{q}^{m^{\prime}, \nu^{\prime}}$ or $\mathcal{P}_{q}^{m^{\prime}, \nu^{\prime}}$ ). Denote by $H_{a}^{1}, H_{b}^{b},-1 \leq a, b \leq 1$, the functions given by any of the formulas (3.2.1). We have to compute $\left\{H_{a}^{A}(U, U), H_{b}^{B}(U, U)\right\}$. According to (3.2.2), the first quantity to study is given by (3.2.3) or (3.2.4) with $W=U$. They may be written as expressions of type

$$
\int_{X}(C(U) u) \bar{u} d \mu, \int_{X}(C(U) u) u d \mu, \int_{X}(C(U) \bar{u}) \bar{u} d \mu .
$$

Consider first the contribution to the left hand side of (3.2.11) given by (3.2.3), (3.2.4). Then $C$ may be expressed from the composition of an element of $H \Psi_{p}^{m, \nu}$ and of $H \Psi_{q}^{m^{\prime}, \nu^{\prime}}$. By lemma 1.2.4 with $a=\max \left(\nu, \nu^{\prime}\right)$ and (3.2.10), we obtain that $C$ is in $H \Psi_{p+q}^{m+m^{\prime}, \min \left(\nu, \nu^{\prime}\right)}$ if $p>0, q>0$ and $H \Psi_{p+q}^{m+m^{\prime}, \nu+\nu^{\prime}}$ if $p=0$ or $q=0$. This shows that (3.2.30) belongs to the right hand side of (3.2.11). If we consider the contribution of (3.2.4) to (3.2.12), we get expressions of form (3.2.30) with $C=[A, B]$. We apply Proposition 2.2.6 (iii), which is possible under assumption (3.2.10), and see that $C$ is in $H \Psi_{p+q}^{m+m^{\prime}-1, \min \left(\nu, \nu^{\prime}\right)+M_{0}(3 d+4)}$ if $p>0, q>0$ and $H \Psi_{p+q}^{m+m^{\prime}-1, \nu+\nu^{\prime}+2 M_{0}(2 d+3)}$ if $p=0$ or $q=0$.

Let us study as well the contributions of (3.2.3), (3.2.4) to the left hand side of (3.2.13), (3.2.14). For the first of these inclusions, $C$ may be expressed from the composition of an element of $\mathcal{P}_{p}^{m, \nu}$ 
and $\mathcal{P}_{q}^{m^{\prime}, \nu^{\prime}}$, so belongs to $\mathcal{P}_{p+q}^{m+m^{\prime}, \min \left(\nu, \nu^{\prime}\right)}$ (if $p>0, q>0$ ) and $\mathcal{P}_{p+q}^{m+m^{\prime}, \nu+\nu^{\prime}}$ if $p=0$ or $q=0$ by (1.2.14), so that (3.2.30) contributes to the right hand side of inclusion (3.2.13). The inclusion (1.2.10) shows that the contributions of (3.2.3), (3.2.4) to the left hand side of (3.2.14) belong to $\mathcal{G}_{p+q}^{m+m^{\prime}, \min \left(\nu, \nu^{\prime}\right)+2 M_{0}}\left(B_{\sigma}(R)\right)$ when if $p>0, q>0$ and to $\mathcal{G}_{p+q}^{m+m^{\prime}, \nu+\nu^{\prime}+2 M_{0}}\left(B_{\sigma}(R)\right)$ if $p=0$ or $q=0$.

We consider now the contributions of type (3.2.5) to the inclusions (3.2.11)-(3.2.14) (as well as the symmetric ones obtained exchanging $A$ and $B$ ). According to (3.2.7), we must study

$$
\left.\int_{X}\left(d_{(w, \bar{w})} A(W) \cdot C(W) u_{1}\right) u_{2} d \mu\right|_{W=U},
$$

where $u_{1}, u_{2}$ stand for $u$ or $\bar{u}, C(W)$ stands for $B(W)$ or ${ }^{t} B(W)$ or $B(W)+{ }^{t} B(W)$, and the symmetric expressions obtained exchanging $A$ and $B$.

Let us study first contributions of type (3.2.31) to the left hand side of (3.2.11), (3.2.12). Then $A$ is in $H \Psi_{p}^{m, \nu}$ and $C$ is in $H \Psi_{q}^{m^{\prime}, \nu^{\prime}} \subset \mathcal{P}_{q}^{m^{\prime}, \nu^{\prime}+2 M_{0}}$. It follows from Lemma 3.2.4 that (3.2.31) is in $H \mathcal{F}_{p+q}^{m, m_{+}^{\prime}+\min \left(\nu, \nu^{\prime}\right)}\left(B_{\sigma}(R)\right)$ if $p>0, q>0$ and to $H \mathcal{F}_{p+q}^{m, m_{+}^{\prime}+\nu+\nu^{\prime}+2 M_{0}}\left(B_{\sigma}(R)\right)$ if $p=0$ or $q=0$. Consider now (3.2.13). Then $A$ (resp. $C$ ) in $(3.2 .31)$ is in $\mathcal{P}_{p}^{m, \nu}$ (resp. $\mathcal{P}_{q}^{m^{\prime}, \nu^{\prime}}$ ) so that Lemma 3.2.4 shows that (3.2.31) is in $\mathcal{G}_{p+q}^{m, m_{+}^{\prime}+\min \left(\nu, \nu^{\prime}\right)}\left(B_{\sigma}(R)\right)$ if $p>0, q>0$ and in $\mathcal{G}_{p+q}^{m, m_{+}^{\prime}+\nu+\nu^{\prime}}\left(B_{\sigma}(R)\right)$ when $p=0$ or $q=0$. For inclusion (3.2.14), we use that in (3.2.31), $A$ is in $H \Psi_{p}^{m, \nu}$ and $B$ in $\mathcal{P}_{q}^{m^{\prime}, \nu^{\prime}}$, so that Lemma 3.2.4 shows that $(3.2 .31)$ is in $H \mathcal{F}_{p+q}^{m, m_{+}^{\prime}+\min \left(\nu, \nu^{\prime}\right)}\left(B_{\sigma}(R)\right)$ when $p>0, q>0$ and in $H \mathcal{F}_{p+q}^{m, m_{+}^{\prime}+\nu+\nu^{\prime}}\left(B_{\sigma}(R)\right)$ when $p=0$ or $q=0$.

Those contributions coming from the symmetric version of (3.2.31) with $A$ and $B$ exchanged belong to the space of functions obtained exchanging $(m, \nu)$ and $\left(m^{\prime}, \nu^{\prime}\right)$, except for inclusion (3.2.14). In this case, we have to consider (3.2.31) with $A$ replaced by an element of $\mathcal{P}_{q}^{m^{\prime}, \nu^{\prime}}$ and $C$ by an element of $H \Psi_{p}^{m, \nu} \subset \mathcal{P}_{q}^{m, \nu+2 M_{0}}$. Again, by Lemma 3.2.4, we get a contribution belonging to $\mathcal{G}_{p+q}^{m^{\prime}, m_{+}+\min \left(\nu, \nu^{\prime}\right)}\left(B_{\sigma}(R)\right)$ if $p>0, q>0$ and to $\mathcal{G}_{p+q}^{m^{\prime}, m_{+}+\nu+\nu^{\prime}+2 M_{0}}\left(B_{\sigma}(R)\right)$ if $p$ or $q$ is zero.

Finally, we have to study the last contribution to (3.2.2), namely $\left\{H_{a}^{A}(\cdot, U), H_{b}^{B}(\cdot, U)\right\}$. We decompose

$$
H_{a}^{A}(W, U)=\sum_{j=0}^{+\infty} H_{a}^{A, j}(W, U)
$$

where

$$
H_{a}^{A, j}(W, U)=\int_{X}\left(A(W) \Delta_{j} u_{1}\right) u_{2} d \mu
$$

with $\left(u_{1}, u_{2}\right)=(u, u)$ if $a=1,\left(u_{1}, u_{2}\right)=(u, \bar{u})$ if $a=0,\left(u_{1}, u_{2}\right)=(\bar{u}, \bar{u})$ if $a=-1$. We use a similar decomposition for $H_{b}^{B}$ and set $H_{a}^{A,<j}=\sum_{j^{\prime}<j} H_{a}^{A, j^{\prime}}, H_{a}^{A, \leq j}=\sum_{j^{\prime} \leq j} H_{a}^{A, j^{\prime}}$. Then

$$
\left\{H_{a}^{A}(\cdot, U), H_{b}^{B}(\cdot, U)\right\}=\sum_{j}\left\{H_{a}^{A, \leq j}(\cdot, U), H_{b}^{B, j}(\cdot, U)\right\}+\sum_{j}\left\{H_{a}^{A, j}(\cdot, U), H_{b}^{B,<j}(\cdot, U)\right\} .
$$


We study the first sum in the right hand side. The general term in that sum may be written $-i\left(d_{W} H_{b}^{B, j}\right) \cdot\left({ }^{t} J \nabla_{W} H_{a}^{A, j}\right)$ so is equal to

$$
-\left.i \int_{X}\left(\left[d_{W} B(W) \cdot\left({ }^{t} J \nabla_{W} H_{a}^{A, j}(W, U)\right)\right] \Delta_{j} u_{1}\right) u_{2} d \mu\right|_{W=U} \cdot
$$

We apply Lemma 3.2.2 considering $A$ as an element of $\mathcal{P}_{p}^{m, \nu+2 M_{0}}$. This allows us to write (3.2.33) as a linear combination of quantities

$$
\int_{X}\left[\left(d_{U} B(U) \cdot M(U) S_{j} u_{3}\right) \Delta_{j} u_{1}\right] u_{2} d \mu
$$

where $u_{3}=u$ or $\bar{u}$ and $M$ is in $H \mathcal{M}_{p}^{m, \nu+2 M_{0}}$. The sum in $j$ of these quantities may be computed from expressions

$$
\sum_{j} \int_{X}\left(B\left(u_{1}, \ldots, u_{\ell}, M\left(u_{\ell+1}, \ldots, u_{\ell+p}\right) S_{j} u_{\ell+p+1}, u_{\ell+p+2}, \ldots, u_{p+q}\right) \Delta_{j} u_{p+q+1}\right) u_{0} d \mu
$$

where $u_{i}$ has to be replaced by $u$ or $\bar{u}$. This is an expression of the form

$$
\int_{X}\left(C\left(u_{1}, \ldots, u_{p+q}\right) u_{p+q+1}\right) u_{0} d \mu
$$

with $C$ given by (3.2.24) (up to a change of indices). By Lemma 3.2.5, $C$ is in $\widetilde{H \Psi} m_{p+q}^{m^{\prime}, m_{+}+\min \left(\nu, \nu^{\prime}\right)}$ (resp. $\quad \widetilde{\mathcal{P}}_{p+q}^{m^{\prime}, m_{+}+\min \left(\nu, \nu^{\prime}\right)}$ ) if $B$ is in $H \Psi_{q}^{m^{\prime}, \nu^{\prime}}$ (resp. $\mathcal{P}_{q}^{m^{\prime}, \nu^{\prime}}$ ). This gives to (3.2.32) a contribution belonging to $H \mathcal{F}_{p+q}^{m^{\prime}, m_{+}+\min \left(\nu, \nu^{\prime}\right)}\left(B_{\sigma}(R)\right)$, when we consider (3.2.11), (3.2.12) and to $\mathcal{G}_{p+q}^{m^{\prime}, m_{+}+\min \left(\nu, \nu^{\prime}\right)}\left(B_{\sigma}(R)\right)$ for (3.2.13), (3.2.14).

The second contribution to (3.2.32) belongs to the same classes with $(m, \nu)$ and $\left(m^{\prime}, \nu^{\prime}\right)$ exchanged in the case of (3.2.11), (3.2.12), (3.2.13). For (3.2.14), we get a contribution in $H \mathcal{F}_{p+q}^{m, m_{+}^{\prime}+\min \left(\nu, \nu^{\prime}\right)}\left(B_{\sigma}(R)\right)$ (Note that when studying (3.2.32) we may assume $p>0$ and $q>0$, as otherwise the corresponding contribution is zero).

Inclusion (3.2.15) follows from (3.2.13) and the inclusion $H \mathcal{F}_{p}^{m, \nu}\left(B_{\sigma}(R)\right) \subset \mathcal{G}_{p}^{m, \nu+2 M_{0}}\left(B_{\sigma}(R)\right)$.

We shall need a version of inclusion (3.2.12), when one of the functions involved in the bracket is not homogeneous, and with a weaker conclusion.

Lemma 3.2.6 Let $\nu, \nu^{\prime}$ in $\mathbb{R}_{+}, p$ in $\mathbb{N}, q$ in $\mathbb{N}^{*}, R>0$. Assume (2.1.10). There is $s_{0}$, depending only on $\nu, \nu^{\prime}, p, q$ such that if $s \geq s_{0}$, if $\Theta$ is in $H \mathcal{F}_{p, \mathrm{H}}^{2 s, \nu}\left(B_{s}(R)\right)+\mathcal{G}_{p}^{2 s-1, \nu}\left(B_{s}(R)\right)$ and $F$ is in $\mathcal{F}_{q, \mathrm{H}}^{1, \nu^{\prime}}\left(B_{s}(R)\right)$, then $\{\Theta, F\}(U)=O\left(\|U\|_{H^{s}}^{q+2}\right), U \rightarrow 0$.

Proof: If $\Theta$ is in $\mathcal{G}_{p}^{2 s-1, \nu}\left(B_{s}(R)\right),\{\Theta, F\}(U)=d \Theta \cdot X_{F}(U)$ is well defined and is $O\left(\|U\|_{H^{s}}^{q+2}\right)$ since, by the last remark following Definition $1.2 .7, X_{F}(U)$ is in $H^{s-1}$ while $d \Theta$ belongs to $\mathcal{L}\left(H^{s-1}, \mathbb{R}\right)$ because of the definition of $\mathcal{G}_{p}^{2 s-1, \nu}\left(B_{s}(R)\right)$ (if $s$ is large enough). 
Assume from now on that $\Theta$ is in $H \mathcal{F}_{p, \mathrm{H}}^{2 s, \nu}\left(B_{s}(R)\right)$. Using notation (3.2.1), we may write $\Theta(U)=$ $H_{0}^{A}(U, U), F(U)=H_{0}^{B}(U, U)$ with $A$ in $H \Psi_{p}^{2 s, \nu}$ and $B$ in $\Psi_{q}^{1, \nu^{\prime}}\left(B_{s}(R)\right)$. We have seen, in the last remark following Definition 1.2.5, that we may consider $A$ as an element of $\Psi_{0}^{2 s, \bar{\nu}}\left(B_{s}(R)\right)$ with $\bar{\nu}>\nu+\nu(p)+\frac{1}{2}$. In particular, by (iii) of Proposition 2.2.6, $[A, B]$ is an element of $\Psi_{q}^{2 s, \max \left(\bar{\nu}, \nu^{\prime}\right)+M_{0}(3 d+4)}\left(B_{s}(R)\right)$. We use $(3.2 .2)$ to express $\{\Theta, F\}$. The first term in the right hand side of this equality is given by the last formula in (3.2.4). Since $[A, B]$ is of order $2 s$, we do get, for $s \geq s_{0}$ large enough, a $O\left(\|U\|_{H^{s}}^{q+2}\right)$ contribution.

We consider next the second term in the right hand side of (3.2.2). By $(3.2 .6),{ }^{t} J \nabla_{U} H_{0}^{B}(U, U)$ is in $H^{s-1}$, and is $O\left(\|U\|_{H^{s}}^{q+1}\right.$ ) (if $s \geq s_{0}$ large enough) using (1.2.18). The term under study, given by the second formula in (3.2.5), is thus $O\left(\|U\|_{H^{s}}^{q+1}\right)$, since $d_{W} A(W) \cdot\left({ }^{t} J \nabla_{U} H_{0}^{B}(U, U)\right)$ is $\mathcal{L}\left(H^{s}, H^{-s}\right)$ by $(1.2 .18)$.

Take now the third term in the right hand side of (3.2.2). By (3.2.6), ${ }^{t} J \nabla_{U} H_{0}^{A}(U, U)$ is in $H^{-s}$, with norm in that space $O\left(\|U\|_{H^{s}}\right)$. We apply (1.2.19) to $B$, with $m=1, \sigma^{\prime}=-s$. We get, if $s$ is large enough relatively to $\nu(q), \nu^{\prime}$, that $d B(U) \cdot\left({ }^{t} J \nabla_{U} H_{0}^{A}\right) u$ is in $H^{-s}$, with norm in that space $O\left(\|U\|_{H^{s}}^{q+1}\right)$. The corresponding contribution to $\{\Theta, F\}$ is again $O\left(\|U\|_{H^{s}}^{q+1}\right)$.

Finally, the last contribution to (3.2.2) will be expressed from

$$
\left.\int_{X}\left(d_{W} A(W) \cdot\left({ }^{t} J \nabla_{W} H_{0}^{B}(W, U)\right) u\right) \bar{u} d \mu\right|_{W=U}
$$

and from the similar expression exchanging $A$ and $B$. We need to check that

$$
d_{W} A(W) \cdot\left({ }^{t} J \nabla_{W} H_{0}^{B}(W, U)\right), d_{W} B(W) \cdot\left({ }^{t} J \nabla_{W} H_{0}^{A}(W, U)\right)
$$

are in $\mathcal{L}\left(H^{s}, H^{-s}\right)$. This will follow from (1.2.19) with $m=2 s$ (resp. $m=1$ ) if we show that $\nabla_{W} H_{0}^{B}(W, U)$ (resp. $\left.\nabla_{W} H_{0}^{A}(W, U)\right)$ belongs to $H^{\sigma^{\prime}}$ for some $\sigma^{\prime} \geq \bar{\nu}$ (resp. to $H^{-s}$ ) and if $s$ is large enough. In other words, we have to check that

$$
\left.H \rightarrow \int_{X}\left(\left(d_{W} B(W) \cdot H\right) u\right) \bar{u} d \mu\right|_{W=U},\left.H \rightarrow \int_{X}\left(\left(d_{W} A(W) \cdot H\right) u\right) \bar{u} d \mu\right|_{W=U}
$$

are linear continuous on $H^{-\sigma^{\prime}}$ (resp. $H^{s}$ ). This follows again from (1.2.19).

\section{Proof of the main theorem}

We have seen that Theorem 1.2.8 follows from Theorem 3.1.1, Theorem 3.1.2 and Theorem 3.1.3. This section is devoted to the proof of these results.

\subsection{Elimination of elliptic terms of lower degree}

This subsection will be devoted to the proof of Theorem 3.1.1: we shall construct a canonical transformation $\chi$ such that (3.1.3) holds, i.e. such that we may reduce the Hamiltonian $G$ to 
another Hamiltonian $G_{L}+G_{\mathrm{H}}+\tilde{G}_{P}$, where all elliptic terms of degree strictly smaller that $P$ have been eliminated. Let us introduce a class of smoothing operators.

Definition 4.1.1 Let $m \in \mathbb{R}, p \in \mathbb{N}, p \geq 2, \gamma \in \mathbb{R}_{+}, s \geq \gamma, R>0$. One denotes by $\mathcal{R}_{\gamma, p}^{m}\left(B_{s}(R)\right)$ the space of maps $U \rightarrow \mathfrak{R}(U)$, defined on $B_{s}(R)$, with values in $H^{2 s-m-\gamma}$, satisfying the following:

(i) The map $U \rightarrow \mathfrak{R}(U)$ is $C^{1}$ from $B_{s}(R)$ to $H^{2 s-m-\gamma}$ and there is $C>0$ such that, for any $U$ in $B_{s}(R)$

$$
\|\mathfrak{R}(U)\|_{H^{2 s-m-\gamma}} \leq C\|U\|_{H^{s}}^{p} .
$$

(ii) For any $\theta, \theta^{\prime}$ in $\mathbb{R}$ satisfying

$$
s-m \geq|\theta|, s-m \geq\left|\theta^{\prime}\right|, s+\theta+\theta^{\prime}>m+\gamma,
$$

for any $U$ in $B_{s}(R), \partial_{U} \mathfrak{R}(U)$ extends as an element of $\mathcal{L}\left(H^{\theta^{\prime}}, H^{-\theta}\right)$ with estimates

$$
\left\|\partial_{U} \mathfrak{R}(U)\right\|_{\mathcal{L}\left(H^{\theta^{\prime}}, H^{-\theta}\right)} \leq C\|U\|_{H^{s}}^{p-1} .
$$

The main example of remainders satisfying the conditions of the preceding definition is given by the following lemma:

Lemma 4.1.2 Let $m \in \mathbb{R}, \nu \in \mathbb{R}_{+}, p \leq q$ in $\mathbb{N}^{*}$. Let $s, \gamma$ such that

$$
s \geq \gamma>\nu(q)+\nu+\frac{1}{2}, 2 s \geq m+\gamma .
$$

Let $A$ be in $H \Psi_{q}^{m, \nu}$ and define $H(W, U)=\int_{X}\left(A(W) w_{1}\right) w_{2} d \mu$ where $w_{1}$, w $w_{2}$ stand for $u$ or $\bar{u}$. Then $\mathfrak{R}(U)=\left.X_{H(\cdot, U)}\right|_{W=U}=\left.i^{t} J \nabla_{W} H(W, U)\right|_{W=U}$ is in $\mathcal{R}_{\gamma, p+1}^{m}\left(B_{s}(R)\right)$ for any $R>0$.

Proof: Decomposing $A(W)=\sum_{\ell=0}^{q} A_{\ell}(w, \ldots, w, \bar{w}, \ldots, \bar{w})$ with $A_{\ell}$ in $\widetilde{H \Psi}_{q}^{m, \nu}$, we may assume that $A$ is one of the terms in that sum. If $K=(k, \bar{k})$ is a smooth function on $X$, we may write by duality $\int_{X} \mathfrak{R}(U) \cdot K d \mu$ from expressions of type

$$
\int_{X}\left(A\left(J h, u_{2}, \ldots, u_{q}\right) u_{q+1}\right) u_{0} d \mu
$$

where $h$ stands for $k$ or $\bar{k}$ and $u_{\ell}$ is $u$ or $\bar{u}$, and from similar expressions where the $J h$ term replaces any other argument of $A$. In the same way, $\int_{X}\left(\partial_{U} \Re(U) \cdot K^{\prime}\right) K d \mu$ may be written from expressions of type

$$
\begin{gathered}
\int_{X}\left(A\left(J h, h^{\prime}, u_{3}, \ldots, u_{q}\right) u_{q+1}\right) u_{0} d \mu \\
\int_{X}\left(A\left(J h, u_{2}, \ldots, u_{q}\right) h^{\prime}\right) u_{0} d \mu
\end{gathered}
$$


and from similar expressions, where $J h$ replaces any other argument inside $A$, or $h^{\prime}=k^{\prime}$ or $\bar{k}^{\prime}$ replaces any other argument of $A$, or where $h^{\prime}$ and $u_{0}$ are exchanged in (4.1.7). To prove (4.1.1), we must bound the modulus of (4.1.5) by

$$
C\|h\|_{H^{-2 s+\gamma+m}}\left\|u_{0}\right\|_{H^{s}} \prod_{\ell=2}^{q+1}\left\|u_{\ell}\right\|_{H^{s}} .
$$

In the same way, to show (4.1.3), we have to bound the modulus of (4.1.6) and (4.1.7) respectively by

$$
C\|h\|_{H^{\theta}}\left\|h^{\prime}\right\|_{H^{\theta^{\prime}}} \prod_{\ell=3}^{q+1}\left\|u_{\ell}\right\|_{H^{s}}\left\|u_{0}\right\|_{H^{s}}, C\|h\|_{H^{\theta}}\left\|h^{\prime}\right\|_{H^{\theta^{\prime}}} \prod_{\ell=2}^{q}\left\|u_{\ell}\right\|_{H^{s}}\left\|u_{0}\right\|_{H^{s}} .
$$

To obtain (4.1.8), we apply (1.2.9) with $N_{2}=2 s-m$. We obtain a bound of (4.1.5) by the product of $C\left\|u_{0}\right\|_{H^{s}}\left\|u_{q+1}\right\|_{H^{s}}$ and of

$$
\begin{aligned}
\sum_{n_{1}} \cdots \sum_{n_{q}}\left(n_{1}+\cdots+\right. & \left.n_{q}\right)^{\nu(q)+\nu-2 s+m}\left\|\Pi_{n_{1}} h\right\|_{L^{2}} \prod_{\ell=2}^{q}\left\|\Pi_{n_{\ell}} u_{\ell}\right\|_{L^{2}} \\
& \leq C \sum_{n_{1}} \cdots \sum_{n_{q}} n_{1}^{\nu(q)+\nu-\gamma} \prod_{\ell=2}^{q} n_{\ell}^{-s} \prod_{\ell=1}^{q} c_{n_{\ell}}^{\ell}\|h\|_{H^{-2 s+\gamma+m}} \prod_{\ell=2}^{q}\left\|u_{\ell}\right\|_{H^{s}}
\end{aligned}
$$

where $\left(c_{n_{\ell}}^{\ell}\right)_{n_{\ell}}$ are $\ell^{2}$-sequences, and where we used that the exponent in the left hand side of (4.1.10) is negative by (4.1.4). The assumptions on $\gamma, s$ show that the series converges, which gives (4.1.8).

To estimate (4.1.6) by the first term in (4.1.9), we bound the modulus of (4.1.6) by

$$
\sum_{n} \sum_{n^{\prime}} \sum_{n_{3}} \cdots \sum_{n_{q}}\left\|A\left(J \Pi_{n} h, \Pi_{n^{\prime}} h^{\prime}, \Pi_{n_{3}} u_{3}, \ldots, \Pi_{n_{q}} u_{q}\right) u_{q+1}\right\|_{H^{-s}}\left\|u_{0}\right\|_{H^{s}} .
$$

We apply (1.2.9) with $N_{2}=2 s-m$ to get a bound given by the product of the factor $C\|h\|_{H^{\theta}}\left\|h^{\prime}\right\|_{H^{\theta^{\prime}}} \prod_{\ell=3}^{q+1}\left\|u_{\ell}\right\|_{H^{s}}$ and of

$$
\sum_{n} \sum_{n^{\prime}} \sum_{n_{3}} \cdots \sum_{n_{q}}\left(n+n^{\prime}+n_{3}+\cdots+n_{q}\right)^{\nu(q)+\nu-2 s+m} n^{-\theta} n^{\prime-\theta^{\prime}} c_{n} c_{n^{\prime}} \prod_{\ell=3}^{q} n_{\ell}^{-s} c_{n_{\ell}}^{\ell}
$$

with $\ell^{2}$-sequences $\left(c_{n}\right)_{n},\left(c_{n^{\prime}}^{\prime}\right)_{n^{\prime}},\left(c_{n_{\ell}}^{\ell}\right)_{n_{\ell}}$. Since the first exponent is negative and $s>1 / 2$, this sum is finite as soon as

$$
\int_{\mathbb{R}_{+}^{2}}\left(1+X+X^{\prime}\right)^{-\alpha}(1+X)^{-\theta}\left(1+X^{\prime}\right)^{-\theta^{\prime}} c(X) c^{\prime}\left(X^{\prime}\right) d X d X^{\prime}<+\infty
$$

where $c(\cdot), c^{\prime}(\cdot)$ are $L^{2}$ functions, $\alpha=2 s-m-\nu(q)-\nu$. One checks that (4.1.11) holds as soon as

$$
\alpha>\max \left[\left(\frac{1}{2}-\theta\right)_{+}+\left(\frac{1}{2}-\theta^{\prime}\right),\left(\frac{1}{2}-\theta\right)+\left(\frac{1}{2}-\theta^{\prime}\right)_{+}\right]
$$

which follows from assumptions (4.1.2), (4.1.4). 
Let us study (4.1.7). We bound the modulus of this quantity by

$$
\sum_{n} \sum_{n_{2}} \cdots \sum_{n_{q}}\left\|A\left(J \Pi_{n} h, \Pi_{n_{2}} u_{2}, \ldots, \Pi_{n_{q}} u_{q}\right) h^{\prime}\right\|_{H^{-s}}\left\|u_{0}\right\|_{H^{s}} .
$$

We apply estimate (1.2.9) with $N_{2}=s+\theta^{\prime}-m \geq 0$ and $s$ replaced by $\theta^{\prime}$, and get a bound given by the product of $C\|h\|_{H^{\theta}}\left\|h^{\prime}\right\|_{H^{\theta^{\prime}}} \prod_{2}^{q}\left\|u_{\ell}\right\|_{H^{s}}\left\|u_{0}\right\|_{H^{s}}$ and of

$$
\sum_{n} \sum_{n_{2}} \cdots \sum_{n_{q}}\left(n+n_{2}+\cdots+n_{q}\right)^{\nu(q)+\nu-s-\theta^{\prime}+m} n^{-\theta} c_{n} \prod_{2}^{q} n_{\ell}^{-s} c_{n_{\ell}}^{\ell}
$$

for $\ell^{2}$-sequence $\left(c_{n}\right)_{n},\left(c_{n_{\ell}}^{\ell}\right)_{n_{\ell}}$. By symmetry, we may assume $n_{2} \geq \cdots \geq n_{q}$. This reduces the verification of the finiteness of (4.1.13) to the study of an integral of form (4.1.11) with $\left(\alpha, \theta, \theta^{\prime}\right)$ replaced by $\left(s+\theta^{\prime}-m-\nu(q)-\nu, \theta, s\right)$. One checks that condition (4.1.12) follows from (4.1.2), (4.1.4). This concludes the proof.

To prepare the proof of Theorem 3.1.1, we have to study the Hamiltonian flow of some auxiliary functions. Let $A_{p}$ be an element of $H \Psi_{p}^{0, \nu}, 1 \leq p \leq P-1$, for some $\nu \in \mathbb{R}_{+}$. Define $A=\sum_{p=1}^{P-1} A_{p}$, and set

$$
F(U)=\operatorname{Re} \int_{X}(A(U) u) u d \mu
$$

By (1.1.16) and Lemma 4.1.2

$$
X_{F}(U)=\frac{i}{2}\left[\begin{array}{c}
\left(\overline{A(U)}+{ }^{t} \overline{A(U)}\right) \bar{u} \\
-\left(A(U)+{ }^{t} A(U)\right) u
\end{array}\right]+\mathfrak{R}(U)=\mathcal{A}(U) U+\mathfrak{R}(U)
$$

where $\mathcal{A}(U)=\frac{i}{2}\left[\begin{array}{cc}0 & \bar{A}+{ }^{t} \bar{A} \\ -\left(A+{ }^{t} A\right) & 0\end{array}\right]$ and $\mathfrak{R}$ is an element of $\mathcal{R}_{\gamma, 2}^{0}\left(B_{\sigma}(R)\right)$ if $\gamma>\nu(P-1)+\nu+\frac{1}{2}$, $\sigma>\gamma$. By the Cauchy-Lipschitz theorem, the equation

$$
\begin{aligned}
\dot{\Phi}(t, U) & =X_{F}(\Phi(t, U)) \\
\Phi(0, U) & =U
\end{aligned}
$$

where $\Phi(t, U)=\left[\frac{\phi(t, U)}{\phi(t, U)}\right]$, has a unique solution defined for $t \in[-1,1]$, for $U$ in $B_{\sigma}(R)$, with $R$ small enough. Moreover, $\Phi(t, U)$ stays in a bounded subset of $H^{s}$ if $U$ stays in $B_{s}(R)$ for some $s \geq \sigma$ and $t \in[-1,1]$.

Let us notice that if $s \geq \sigma$ and $U$ is in $B_{s}(R), D \Phi(t, U)$ extends as an element of $\mathcal{L}\left(H^{s^{\prime}}, H^{s^{\prime}}\right)$ for any $s^{\prime}$ with $\left|s^{\prime}\right| \leq s$, any $t$ in $[-1,1]$. Actually, consider the solution $W(t, U)$ of the linear ODE

$$
\begin{aligned}
\dot{W}(t, U) & =\mathcal{A}(\Phi(t, U)) W(t, U)+[D \mathcal{A}(\Phi(t, U)) \cdot W(t, U)] \Phi(t, U)+D \mathfrak{R}(\Phi(t, U)) \cdot W(t, U) \\
W(0, U) & =\mathrm{Id} .
\end{aligned}
$$

We just need to check that the right hand side of the first equation in (4.1.16) is, as a function of $W$, a bounded linear map from $\mathcal{L}\left(H^{s^{\prime}}, H^{s^{\prime}}\right)$ to itself, for any $s^{\prime}$ with $\left|s^{\prime}\right| \leq s$, when $U$ is taken in $B_{s}(R)$. The boundedness of $W \rightarrow D \Re(\Phi(t, U)) \cdot W$ follows from (4.1.3), since $\Phi(t, U)$ stays 
in a bounded subset of $H^{s}$ and (4.1.2) holds by the conditions imposed to $\sigma, \gamma$. The continuity of $W \rightarrow \mathcal{A}(\Phi(t, U)) W$ is a consequence of (1.2.9) and the fact that $\sigma>\nu(P-1)+\nu+\frac{1}{2}$. We are left with examining the middle term in the right hand side of the first equation (4.1.16). Let $\bar{\nu}=\nu+\nu(P-1)-\nu(1)+\frac{1}{2}+0$. By the last remark following Definition 1.2.5, $\mathcal{A}$ is in $\Psi_{1}^{0, \bar{\nu}}\left(B_{\sigma}(R)\right) \otimes \mathcal{M}_{2}(\mathbb{R})$. We write for $H$ in $H^{s^{\prime}}$

$$
\begin{aligned}
\| D \mathcal{A}(\Phi(t, U)) \cdot(W \cdot H) \Phi(t, U)) \|_{H^{s^{\prime}}} & \leq C \| D \mathcal{A}(\Phi(t, U)) \cdot(W \cdot H))\left\|_{\mathcal{L}\left(H^{s}, H^{s^{\prime}}\right)}\right\| \Phi(t, U) \|_{H^{s}} \\
& \leq C\|W \cdot H\|_{H^{s^{\prime}}}\|\Phi(t, U)\|_{H^{s}},
\end{aligned}
$$

where the last inequality comes from (1.2.19) applied with $\sigma=s, \sigma^{\prime}=s^{\prime}$, and noticing that $s-\left(\nu(1)+\bar{\nu}-s^{\prime}\right)_{+} \geq s^{\prime}$ because if the assumptions on $s, s^{\prime}$. We get the wanted inequality

$$
\|(D \mathcal{A}(\Phi(t, U)) \cdot W) \Phi(t, U))\left\|_{\mathcal{L}\left(H^{s^{\prime}}, H^{s^{\prime}}\right)} \leq C\right\| W \|_{\mathcal{L}\left(H^{s^{\prime}}, H^{s^{\prime}}\right)} .
$$

This gives the fact that $D \Phi(t, U)$ is bounded in $\mathcal{L}\left(H^{s^{\prime}}, H^{s^{\prime}}\right)$ uniformly for $t \in[-1,1]$, if $\left|s^{\prime}\right| \leq s$. We deduce from this and from (1.2.18), (1.2.19):

Corollary 4.1.3 If $s>\gamma>\nu(P-1)+\nu+\frac{1}{2}$ and $\bar{\nu}=\nu+\nu(P-1)-\nu(1)+\frac{1}{2}+0,(\mathcal{A}(\Phi(t, \cdot)))_{-1 \leq t \leq 1}$ is a bounded family of matrices of elements of $\Psi_{1}^{0, \bar{\nu}}\left(B_{s}(R)\right)$.

Let us describe the structure of $\Phi(t, U)$.

Proposition 4.1.4 Let $P \in \mathbb{N}^{*}, s \geq \sigma>\gamma>\nu(P-1)+\nu+\frac{1}{2}$. Let $A$ be in $\sum_{p=1}^{P-1} H \Psi_{p}^{0, \nu} \subset$ $\Psi_{1}^{0, \bar{\nu}}\left(B_{\sigma}(R)\right)$ with $\bar{\nu}=\nu+\nu(P-1)-\nu(1)+\frac{1}{2}+0$. Let $\Phi$ be the solution of (4.1.15), defined for $U$ in $B_{s}(R)$ for some $R>0$. If $R$ is small enough, there is a bounded family $(B(t, \cdot))_{-1 \leq t \leq 1}$ (resp. $\left.(\tilde{\mathfrak{R}}(t, \cdot))_{-1 \leq t \leq 1}\right)$ of $2 \times 2$-matrices of elements of $\Psi_{1}^{0, \bar{\nu}}\left(B_{\sigma}(R)\right)$ (resp. of elements of $\mathcal{R}_{\gamma, 2}^{0}\left(B_{\sigma}(R)\right)$ ) such that, for any $t \in[0,1]$

$$
\Phi(t, U)=U+B(t, U) U+\tilde{\mathfrak{R}}(t, U) .
$$

Proof: By (4.1.15), (4.1.14),

$$
\Phi(t, U)=U+t \int_{0}^{1} \mathcal{A}\left(\Phi\left(\alpha_{1} t, U\right)\right) \Phi\left(\alpha_{1} t, U\right) d \alpha_{1}+t \int_{0}^{1} \mathfrak{R}\left(\Phi\left(\alpha_{1} t, U\right)\right) d \alpha_{1} .
$$

If $R>0$ is small enough, there is $C>0$ such that $\|\Phi(t, U)\|_{H^{s}} \leq C\|U\|_{H^{s}}$ for any $U$ in $B_{s}(R)$, any $t \in[-1,1]$. Iterating (4.1.18), we get

$$
\Phi(t, U)=U+\sum_{k=1}^{N} t^{k} \mathcal{A}^{[k]}(t, U) U+t^{N+1} C_{N}(t, U)+\sum_{k=1}^{N+1} t^{k} \mathfrak{R}^{[k]}(t, U)
$$


where

$$
\begin{aligned}
\mathcal{A}^{[k]}(t, U) & =\int_{0}^{1} \cdots \int_{0}^{1} \prod_{j=1}^{k} \mathcal{A}\left(\Phi\left(t \alpha_{1} \cdots \alpha_{j}, U\right)\right) \prod_{j=1}^{k-1} \alpha_{j}^{k-j} d \alpha_{1} \cdots d \alpha_{k} \\
C_{N}(t, U) & =\int_{0}^{1} \cdots \int_{0}^{1} \prod_{j=1}^{N+1} \mathcal{A}\left(\Phi\left(t \alpha_{1} \cdots \alpha_{j}, U\right)\right) \prod_{j=1}^{N} \alpha_{j}^{N+1-j} \Phi\left(t \alpha_{1} \cdots \alpha_{N+1}, U\right) d \alpha_{1} \cdots d \alpha_{N+1} \\
\mathfrak{R}^{[k]}(t, U) & =\int_{0}^{1} \cdots \int_{0}^{1} \prod_{j=1}^{k-1} \mathcal{A}\left(\Phi\left(t \alpha_{1} \cdots \alpha_{j}, U\right)\right) \prod_{j=1}^{k} \alpha_{j}^{k-j} \mathfrak{R}\left(\Phi\left(t \alpha_{1} \cdots \alpha_{k}, U\right)\right) d \alpha_{1} \cdots d \alpha_{k} .
\end{aligned}
$$

We apply Lemma 1.2.6 with $m=m^{\prime}=0, p=p^{\prime}=1, \nu=\nu^{\prime}=\bar{\nu}$. For some $\mathcal{P}=\left(P_{1}, \ldots, P_{k}\right)$ as in the statement of this lemma, we set for short $\mathfrak{N}_{\sigma}^{\ell, s}=\mathfrak{N}_{(\mathcal{P}, 1, \bar{\nu}, \sigma)}^{\ell, 0, s}$. Then, if $\left|\sigma^{\prime}\right| \leq \sigma, s \in \mathbb{R}$, there is $C>0$ such that for any $U$ in $B_{\sigma}(R)$, any $t \in[0,1]$

$$
\begin{aligned}
& \mathfrak{N}_{\sigma}^{0, s}\left(\mathcal{A}^{[k]}(t, U)\right) \leq \frac{C^{k}}{k !}\left[\mathfrak{N}_{\sigma}^{0, s}(A)\right]^{k} \\
& \mathfrak{N}_{\sigma^{\prime}}^{1, s}\left(\mathcal{A}^{[k]}(t, U)\right) \leq \frac{C^{k}}{k !}\left[\mathfrak{N}_{\sigma}^{0, s}(A)\right]^{k-1} \mathfrak{N}_{\sigma^{\prime}}^{1, s}(A)
\end{aligned}
$$

so that $\sum_{k=1}^{+\infty} t^{k} \mathcal{A}^{[k]}(t, U)$ converges to an element $B(t, \cdot)$ of $\Psi_{1}^{0, \bar{\nu}}\left(B_{\sigma}(R)\right)$. Since $\mathfrak{N}_{\sigma}^{0, s}\left(C_{N}(t, U)\right)=$ $O\left(\frac{C^{N+1}}{(N+1) !} \mathfrak{N}_{\sigma}^{0, s}(A)^{N+1}\right)$, the last but one term in (4.1.19) goes to zero.

Let us check that the last series in (4.1.19) converges in the space $\mathcal{R}_{\gamma, 2}^{0}\left(B_{s}(R)\right)$.

Note first that by (4.1.1) and (1.2.18) $\left\|\mathfrak{R}^{[k]}(t, U)\right\|_{H^{2 s-\gamma}} \leq \frac{C^{k}}{k !}\|U\|_{H^{s}}^{2}$ for $U$ in $B_{s}(R)$, whence an estimate of type (4.1.1) for $\sum_{k} t^{k} \mathfrak{R}^{[k]}(t, U)$, uniformly for $t \in[-1,1]$. Let us study the norm $\left\|\partial_{U} \mathfrak{R}^{[k]}(t, U)\right\|_{\mathcal{L}\left(H^{\left.\theta^{\prime}, H^{-\theta}\right)}\right.}$ for $\theta, \theta^{\prime}$ satisfying (4.1.2). If, when computing $\partial_{U} \mathfrak{R}^{[k]}$ from its expression (4.1.20), the derivative $\partial_{U}$ falls on $\mathfrak{R}\left(\Phi\left(\alpha_{1} \ldots \alpha_{k} t, U\right)\right)$, it follows from (4.1.3), the boundedness of $\partial_{U} \Phi$ on $H^{\theta^{\prime}}$ for $\left|\theta^{\prime}\right| \leq s$, and (1.2.18) that the corresponding contribution to $\left\|\partial_{U} \mathfrak{R}^{[k]}(t, U)\right\|_{\mathcal{L}\left(H^{\theta^{\prime}}, H^{-\theta}\right)}$ is bounded from above by $\frac{C^{k}}{k !}\|U\|_{H^{s}}$. On the other hand, if the derivative falls on $\mathcal{A}\left(\Phi\left(\alpha_{1} \ldots \alpha_{j} t, U\right)\right)$, we have to estimate for $H^{\prime}$ in $H^{\theta^{\prime}}$

$$
\left\|\left(\partial_{U} A\right)\left(\Phi\left(t \alpha_{1} \cdots \alpha_{j}, U\right)\right) \cdot\left(\partial_{U} \Phi\left(t \alpha_{1} \cdots \alpha_{j}, U\right) \cdot H^{\prime}\right)\right\|_{\mathcal{L}\left(H^{2 s-\gamma}, H^{-\theta}\right)}
$$

since $\mathfrak{R}(\Phi(t, \cdot))$ is in $H^{2 s-\gamma}$ by (4.1.1). We apply (1.2.19) with $\sigma=s, \sigma^{\prime}=\theta^{\prime}$. This allows us to estimate (4.1.21) for $H^{\prime} \in H^{\theta^{\prime}}$ if $2 s-\gamma-\left(\nu(1)+\bar{\nu}-\theta^{\prime}\right)_{+} \geq-\theta$, which follows from (4.1.2), the definition of $\bar{\nu}$ and the assumptions of the proposition. Again, we get convergence of the corresponding series. This concludes the proof.

Proof of Theorem 3.1.1: We consider a function $G(U)=\sum_{p=0}^{P^{\prime}} G_{p}(U)$ as in the statement of Theorem 1.2.8. For $p=1, \ldots, P-1, G_{p}$ is an element of $H \mathcal{F}_{p}^{1, \nu^{\prime}}\left(B_{\sigma}(R)\right)$ for some $\nu^{\prime}$. We shall construct the canonical transformation $\chi$ through a standard Birkhoff normal forms method. We use an auxiliary function $F(U)=\sum_{p=1}^{P-1} F_{p}(U)$ with

$$
F_{p}(U)=\operatorname{Re} \int_{X}\left(A_{p}(U) u\right) u d \mu
$$


with $A_{p}$ element of $H \Psi_{p}^{0, \nu}$ for some $\nu \geq \nu^{\prime}$ to be chosen. Let us assume that the increasing function $p \rightarrow \nu(p)$ satisfies

$$
\nu(p)+\nu(q)+\nu+M_{0}(3 d+4) \leq \nu(p+q) \text { if } p>0, q>0 .
$$

We denote by $\Phi$ the flow of (4.1.15) defined from $F$ on $B_{s}(R)$ where $s>\nu(P-1)+\nu+\frac{1}{2}$. We set $\chi(U)=\Phi(-1, U)$ : this is a canonical transformation close to zero, with $\chi(0)=0, \chi^{\prime}(0)=\mathrm{Id}$. For $U$ in $B_{s}(R) \cap C^{\infty}$, we compute

$$
\begin{aligned}
G \circ \chi^{-1}(U) & =G(\Phi(1, U)) \\
& =\left.\sum_{k=0}^{P-1} \frac{1}{k !} \frac{d^{k}}{d t^{k}}(G \circ \Phi(t, U))\right|_{t=0}+\int_{0}^{1} \frac{(1-t)^{P-1}}{(P-1) !} \frac{d^{P}}{d t^{P}}(G \circ \Phi(t, U)) d t \\
& =\sum_{k=0}^{P-1} \frac{(-1)^{k}}{k !} \operatorname{ad}^{k} F \cdot G+\frac{(-1)^{P}}{(P-1) !} \int_{0}^{1}(1-t)^{P-1}\left(\operatorname{ad}^{P} F \cdot G\right)(\Phi(t, U)) d t,
\end{aligned}
$$

using the notation $\operatorname{ad} F \cdot G=\{F, G\}$ and $\operatorname{ad}^{p} F \cdot G=\left\{F, \operatorname{ad}^{p-1} F \cdot G\right\}$. We decompose

$$
\sum_{k=0}^{P-1} \frac{(-1)^{k}}{k !} \operatorname{ad}^{k} F \cdot G=G_{0}-\sum_{\ell=1}^{P-1}\left\{F_{\ell}, G_{0}\right\}+\sum_{\ell \geq 1} H_{\ell}
$$

where the last sum is finite, and where $H_{\ell}$ is a linear combination of quantities of the form

$$
\operatorname{ad} F_{i_{1}} \cdots \operatorname{ad} F_{i_{r}} G_{p}
$$

with $1 \leq i_{j}<\ell, p \geq 0, i_{1}+\cdots+i_{r}+p=\ell$ and $r \geq 2$ if $p=0$. Assume

$$
F_{p} \in H \mathcal{F}_{p}^{0, \nu}\left(B_{\sigma}(R)\right) \text { and }\left\{F_{p}, G_{0}\right\} \in H_{\mathcal{F}_{p}^{1, \nu^{\prime}}}\left(B_{\sigma}(R)\right) 1 \leq p \leq P-1 .
$$

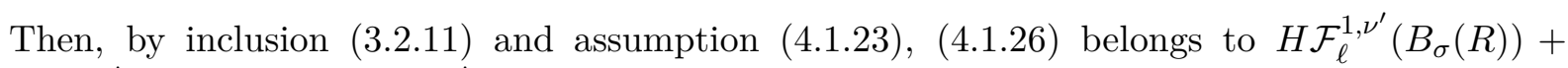
$H \mathcal{F}_{\ell}^{0, \nu^{\prime}+1}\left(B_{\sigma}(R)\right) \subset H \mathcal{F}_{\ell}^{1, \nu^{\prime}}\left(B_{\sigma}(R)\right)$ if $\sigma$ is large enough so that (1.2.23) with $p=\ell$ is satisfied. Consequently, for any $\ell \geq 1, H_{\ell}$ is in $H \mathcal{F}_{\ell}^{1, \nu^{\prime}}\left(B_{\sigma}(R)\right)$, and depends only on $F_{\ell^{\prime}}$ for $\ell^{\prime}<\ell$.

Lemma 4.1.5 Let $\nu \geq \nu^{\prime}+2 M_{0}, \sigma$ satisfying (1.2.23), and denote

$$
\mathcal{G}_{p}^{m-\infty, \nu+\infty}\left(B_{\sigma}(R)\right)=\bigcap_{N \in \mathbb{N}} \mathcal{G}_{p}^{m-N, \nu+N}\left(B_{\sigma}(R)\right)
$$

(with the notations introduced in definition 1.2.7). For each $p=1, \ldots, P-1$ there is $F_{p}$ in $H \mathcal{F}_{p, \mathrm{E}}^{0, \nu}\left(B_{\sigma}(R)\right)$ such that $\left\{F_{p}, G_{0}\right\}-H_{p}$ is in $H \mathcal{F}_{p, \mathrm{H}}^{1, \nu^{\prime}}\left(B_{\sigma}(R)\right)+\mathcal{G}_{p}^{1-\infty, \nu^{\prime}+2 M_{0}+\infty}\left(B_{\sigma}(R)\right)$ and that (4.1.27) holds.

Proof: We decompose $H_{p}=H_{p, \mathrm{E}}+H_{p, \mathrm{H}}$ with $H_{p, \mathrm{E}}$ in $H \mathcal{F}_{p, \mathrm{E}}^{1, \nu^{\prime}}\left(B_{\sigma}(R)\right), H_{p, \mathrm{H}}$ in $H \mathcal{F}_{p, \mathrm{H}}^{1, \nu^{\prime}}\left(B_{\sigma}(R)\right)$. We may write $H_{p, \mathrm{E}}(U)=\operatorname{Re} \int_{X}\left(B_{p}(U) u\right) u d \mu$ with $B_{p}$ in $H \Psi_{p}^{1, \nu^{\prime}}$. By definition of this space

$$
B_{p}(U)=\sum_{\ell=1}^{p} B_{p, \ell}(\underbrace{u, \ldots, u}_{\ell}, \bar{u}, \ldots, \bar{u})
$$


where $B_{p, \ell}$ is in $\widetilde{H \Psi} \Psi^{1, \nu^{\prime}}$. We decompose $B_{p, \ell}=B_{p, \ell}^{(1)}+B_{p, \ell}^{(2)}$ where for any $n^{\prime} \in\left(\mathbb{N}^{*}\right)^{p}, \mathcal{U}=$ $\left(u_{1}, \ldots, u_{p}\right)$,

$$
B_{p, \ell}^{(1)}\left(\Pi_{n^{\prime}} \mathcal{U}\right)=\sum_{j ;\left|n^{\prime}\right| \leq c 2^{j}} B_{p, \ell}\left(\Pi_{n^{\prime}} \mathcal{U}\right) \Delta_{j}
$$

with $c>0$ a small enough constant. By formula (A.1) of the appendix, $B_{p, \ell}^{(1)}$ is in $\widetilde{H \Psi}_{p}^{1, \nu^{\prime}}$. Moreover, if $c$ is small enough,

$$
\Pi_{n_{0}} B_{p, \ell}^{(1)}\left(\Pi_{n^{\prime}} \mathcal{U}\right) \Pi_{n_{p+1}} \not \equiv 0 \Rightarrow \sqrt{\mathbf{m}^{2}+\lambda_{n_{p+1}}^{2}} \geq 2\left(\sum_{1}^{p} \sqrt{\mathbf{m}^{2}+\lambda_{n_{\ell}}^{2}}\right)
$$

and by construction (and the inclusion $(1.2 .10)$ ), $B_{p, \ell}^{(2)}$ is in $\mathcal{G}_{p}^{1-\infty, \nu^{\prime}+2 M_{0}+\infty}\left(B_{\sigma}(R)\right)$. To prove the lemma, we just need to find $F_{p}$ in $H \mathcal{F}_{p, \mathrm{E}}^{0, \nu}\left(B_{\sigma}(R)\right)$ such that

$$
\left\{F_{p}, G_{0}\right\}=\operatorname{Re} \sum_{\ell} \int_{X}\left(B_{p, \ell}^{(1)}(\underbrace{u, \ldots, u}_{\ell}, \bar{u}, \ldots, \bar{u}) u\right) u d \mu .
$$

We look for $F_{p}$ under the form

$$
F_{p}(U)=\sum_{\ell=0}^{p} \operatorname{Re} \int_{X}\left(C_{p, \ell}(u, \ldots, u, \bar{u}, \ldots, \bar{u}) u\right) u d \mu
$$

with $C_{p, \ell}$ in $\widetilde{H \Psi}_{p}^{0, \nu}$. To solve (4.1.29), it is enough to find $C_{p, \ell}$ such that

$$
\begin{aligned}
\sum_{\ell^{\prime}=1}^{\ell} C_{p, \ell}\left(u_{1}, \ldots, \Lambda_{\mathbf{m}} u_{\ell^{\prime}}, \ldots, u_{p}\right)-\sum_{\ell^{\prime}=\ell+1}^{p} C_{p, \ell}\left(u_{1}, \ldots, \Lambda_{\mathbf{m}} u_{\ell^{\prime}}, \ldots, u_{p}\right) \\
+\Lambda_{\mathbf{m}} C_{p, \ell}\left(u_{1}, \ldots, u_{p}\right)+C_{p, \ell}\left(u_{1}, \ldots, u_{p}\right) \Lambda_{\mathbf{m}}=-i B_{p, \ell}^{(1)}\left(u_{1}, \ldots, u_{p}\right) .
\end{aligned}
$$

We replace in the formula $u_{j}$ by $\Pi_{n_{j}} u_{j}$, compose at the left (resp. at the right) with $\Pi_{n_{0}}$ (resp. $\left.\Pi_{n_{p+1}}\right)$. We get

$$
\begin{array}{r}
\tilde{F}_{\mathbf{m}}^{p, \ell}\left(\lambda_{n_{0}}, \ldots, \lambda_{n_{p+1}}\right) \Pi_{n_{0}} C_{p, \ell}\left(\Pi_{n_{1}} u_{1}, \ldots, \Pi_{n_{p}} u_{p}\right) \Pi_{n_{p+1}} \\
=-i \Pi_{n_{0}} B_{p, \ell}^{(1)}\left(\Pi_{n_{1}} u_{1}, \ldots, \Pi_{n_{p}} u_{p}\right) \Pi_{n_{p+1}}
\end{array}
$$

with

$$
\tilde{F}_{\mathbf{m}}^{p, \ell}\left(\xi_{0}, \ldots, \xi_{p+1}\right)=\sqrt{\mathbf{m}^{2}+\xi_{0}^{2}}+\sqrt{\mathbf{m}^{2}+\xi_{p+1}^{2}}+\sum_{\ell^{\prime}=1}^{\ell} \sqrt{\mathbf{m}^{2}+\xi_{\ell^{\prime}}^{2}}-\sum_{\ell^{\prime}=\ell+1}^{p} \sqrt{\mathbf{m}^{2}+\xi_{\ell^{\prime}}^{2}}
$$

We define

$$
C_{p, \ell}\left(u_{1}, \ldots, u_{p}\right)=-i \sum_{n_{0}} \cdots \sum_{n_{p+1}} \tilde{F}_{\mathbf{m}}^{p, \ell}\left(\lambda_{n_{0}}, \ldots, \lambda_{n_{p+1}}\right)^{-1} \Pi_{n_{0}} B_{p, \ell}^{(1)}\left(\Pi_{n_{1}} u_{1}, \ldots, \Pi_{n_{p}} u_{p}\right) \Pi_{n_{p+1}} .
$$


By inclusion (1.2.10), $\Pi_{n_{0}} B_{p, \ell}^{(1)}\left(\Pi_{n^{\prime}} \mathcal{U}\right) \Pi_{n_{p+1}}$ satisfies estimate (1.2.5) with $\nu=\nu^{\prime}+2 M_{0}, m=1$. Since, by (4.1.28), $\left|\tilde{F}_{\mathbf{m}}^{p, \ell}\left(\lambda_{n_{0}}, \ldots, \lambda_{n_{p+1}}\right)\right| \geq c\left(n_{0}+n_{p+1}\right)$ when the right hand side of (4.1.31) is not zero, we get

$$
\begin{aligned}
\left\|\Pi_{n_{0}} C_{p, \ell}\left(\Pi_{n_{1}} u_{1}, \ldots, \Pi_{n_{p}} u_{p}\right) \Pi_{n_{p+1}}\right\|_{\mathcal{L}\left(L^{2}\right)} \leq & C\left\langle n_{0}-n_{p+1}\right\rangle^{-2}\left(\min \left[\frac{n_{0}}{n_{p+1}}, \frac{n_{p+1}}{n_{0}}\right]\right)^{N_{1}} \\
& \times\left|n^{\prime}\right|^{\nu(p)+\nu}\left(1+\frac{\left|n^{\prime}\right|}{n_{0}}\right)^{-N_{2}} \prod_{\ell=1}^{p}\left\|\Pi_{n_{\ell}} u_{\ell}\right\|_{L^{2}}
\end{aligned}
$$

for any $N_{1}, N_{2}$. To prove similar estimates for commutators with differential operators, we consider $P_{1}$ a differential operator of order $d_{1}$ and deduce from (4.1.30)

$$
\begin{aligned}
& \sum_{\ell^{\prime}=1}^{\ell}\left[P_{1}, C_{p, \ell}\right]\left(u_{1}, \ldots, \Lambda_{\mathbf{m}} u_{\ell^{\prime}}, \ldots, u_{p}\right)-\sum_{\ell^{\prime}=\ell+1}^{p}\left[P_{1}, C_{p, \ell}\right]\left(u_{1}, \ldots, \Lambda_{\mathbf{m}} u_{\ell^{\prime}}, \ldots, u_{p}\right) \\
&+\Lambda_{\mathbf{m}}\left[P_{1}, C_{p, \ell}\right]\left(u_{1}, \ldots, u_{p}\right)+\left[P_{1}, C_{p, \ell}\right]\left(u_{1}, \ldots, u_{p}\right) \Lambda_{\mathbf{m}}=-i \tilde{B}_{p, \ell}^{(1)}\left(u_{1}, \ldots, u_{p}\right)
\end{aligned}
$$

where

$$
\begin{array}{r}
\tilde{B}_{p, \ell}^{(1)}\left(u_{1}, \ldots, u_{p}\right)=-i\left[P_{1}, \Lambda_{\mathbf{m}}\right] C_{p, \ell}\left(u_{1}, \ldots, u_{p}\right)-i C_{p, \ell}\left(u_{1}, \ldots, u_{p}\right)\left[P_{1}, \Lambda_{\mathbf{m}}\right] \\
+\left[P_{1}, B_{p, \ell}^{(1)}\right]\left(u_{1}, \ldots, u_{p}\right) .
\end{array}
$$

We need to prove

$$
\begin{aligned}
\left\|\Pi_{n_{0}}\left[P_{1}, C_{p, \ell}\right]\left(\Pi_{n_{1}} u_{1}, \ldots, \Pi_{n_{p}} u_{p}\right) \Pi_{n_{p+1}}\right\|_{\mathcal{L}\left(L^{2}\right)} & \\
\leq C\left\langle n_{0}-n_{p+1}\right\rangle^{-2} n_{0}^{d_{1}-1}\left(\min \left[\frac{n_{0}}{n_{p+1}}, \frac{n_{p+1}}{n_{0}}\right]\right)^{N_{1}}\left|n^{\prime}\right|^{\nu(p)+\nu+M_{0}} & \\
& \times\left(1+\frac{\left|n^{\prime}\right|}{n_{0}}\right)^{-N_{2}} \prod_{\ell=1}^{p}\left\|\Pi_{n_{\ell}} u_{\ell}\right\|_{L^{2}}
\end{aligned}
$$

for any $N_{1}, N_{2}$. Since $B_{p, \ell}^{(1)}$ is in $\widetilde{H \Psi}_{p}^{1, \nu^{\prime}},\left[P_{1}, B_{p, \ell}^{(1)}\right]$ is in $\widetilde{H \Psi}_{p}^{d_{1}, \nu^{\prime}+M_{0}}$ by Definition 1.2 .2 , so in $\widetilde{\mathcal{P}}_{p}^{d_{1}, \nu+M_{0}}$ by inclusion (1.2.10). Consequently

$$
\begin{aligned}
\left\|\Pi_{n_{0}}\left[P_{1}, B_{p, \ell}^{(1)}\right]\left(\Pi_{n_{1}} u_{1}, \ldots, \Pi_{n_{p}} u_{p}\right) \Pi_{n_{p+1}}\right\|_{\mathcal{L}\left(L^{2}\right)} & \\
\leq C\left\langle n_{0}-n_{p+1}\right\rangle^{-2} n_{0}^{d_{1}}\left(\min \left[\frac{n_{0}}{n_{p+1}}, \frac{n_{p+1}}{n_{0}}\right]\right)^{N_{1}}\left|n^{\prime}\right|^{\nu(p)+\nu+M_{0}} & \\
& \times\left(1+\frac{\left|n^{\prime}\right|}{n_{0}}\right)^{-N_{2}} \prod_{\ell=1}^{p}\left\|\Pi_{n_{\ell}} u_{\ell}\right\|_{L^{2}} .
\end{aligned}
$$

Since $\left[P_{1}, \Lambda_{\mathbf{m}}\right]$ is a pseudo-differential operator of order $d_{1}$, we have estimates

$$
\left\|\Pi_{n_{1}}\left[P_{1}, \Lambda_{\mathbf{m}}\right] \Pi_{n_{2}}\right\|_{\mathcal{L}\left(L^{2}\right)} \leq C_{N}\left\langle n_{1}-n_{2}\right\rangle^{-N} n_{1}^{d_{1}}
$$

for any $N$. Combining this with (4.1.32), we conclude that the right hand side of (4.1.33) is such that $\left\|\Pi_{n_{0}} \tilde{B}_{p, \ell}^{(1)}\left(\Pi_{n^{\prime}}(\mathcal{U})\right) \Pi_{n_{p+1}}\right\|_{\mathcal{L}\left(L^{2}\right)}$ is bounded from above by the right hand side of (4.1.35). 
If we use (4.1.36) for $P_{1}$ instead of $\left[P_{1}, \Lambda_{\mathbf{m}}\right]$ and (4.1.32), we conclude that $P_{1} C_{p, \ell}, C_{p, \ell} P_{1}$ and so $\left[P_{1}, C_{p, \ell}\right]$ satisfy estimate (4.1.32) in which we replace, in the right hand side, $\left|n^{\prime}\right|^{\nu(p)+\nu}$ by $\left|n^{\prime}\right|^{\nu(p)+\nu} n_{0}^{d_{1}}$. We decompose $\left[P_{1}, C_{p, \ell}\right]=C_{p, \ell}^{(1)}+C_{p, \ell}^{(2)}$ where

$$
C_{p, \ell}^{(1)}=\sum_{\left|n^{\prime}\right| \leq c n_{p+1}} \Pi_{n_{0}}\left[P_{1}, C_{p, \ell}\right]\left(\Pi_{n^{\prime}} \mathcal{U}\right) \Pi_{n_{p+1}} .
$$

Then $C_{p, \ell}^{(2)}$ satisfies (4.1.35) with $n_{0}^{d_{1}}$ replaced by $n_{0}^{d_{1}-1}$ in the right hand side, because of $\left|n^{\prime}\right| \geq$ $c n_{p+1}$, so that

$$
\begin{array}{r}
\Pi_{n_{0}} \Lambda_{\mathbf{m}} C_{p, \ell}^{(2)}\left(\Pi_{n^{\prime}} \mathcal{U}\right) \Pi_{n_{p+1}}, \Pi_{n_{0}} C_{p, \ell}^{(2)}\left(\Pi_{n^{\prime}} \mathcal{U}\right) \Lambda_{\mathbf{m}} \Pi_{n_{p+1}}, \\
\Pi_{n_{0}} C_{p, \ell}^{(2)}\left(\Pi_{n_{1}} u_{1}, \ldots, \Pi_{n_{\ell^{\prime}}} \Lambda_{\mathbf{m}} u_{\ell^{\prime}}, \ldots, \Pi_{n_{p}} u_{p}\right) \Pi_{n_{p+1}}
\end{array}
$$

satisfy (4.1.35). We obtain in that way from (4.1.33) an equation

$$
\begin{aligned}
\sum_{\ell^{\prime}=1}^{\ell} C_{p, \ell}^{(1)}\left(u_{1}, \ldots,\right. & \left.\Lambda_{\mathbf{m}} u_{\ell^{\prime}}, \ldots, u_{p}\right)-\sum_{\ell^{\prime}=\ell+1}^{p+1} C_{p, \ell}^{(1)}\left(u_{1}, \ldots, \Lambda_{\mathbf{m}} u_{\ell^{\prime}}, \ldots, u_{p}\right) \\
& +\Lambda_{\mathbf{m}} C_{p, \ell}^{(1)}\left(u_{1}, \ldots, u_{p}\right)+C_{p, \ell}^{(1)}\left(u_{1}, \ldots, u_{p}\right) \Lambda_{\mathbf{m}}=-i \tilde{B}_{p, \ell}^{(2)}\left(u_{1}, \ldots, u_{p}\right),
\end{aligned}
$$

where $\left\|\Pi_{n_{0}} \tilde{B}_{p, \ell}^{(2)}\left(\Pi_{n^{\prime}} \mathcal{U}\right) \Pi_{n_{p+1}}\right\|_{\mathcal{L}\left(L^{2}\right)}$ is bounded by the right hand side of (4.1.35) and where, if the constant $c$ in the definition of $C_{p, \ell}^{(1)}$ is small enough, this operator satisfies a condition of type (4.1.28). We may thus solve (4.1.37) in the same way as (4.1.30) and find $C_{p, \ell}^{(1)}$ and $C_{p, \ell}$ such that (4.1.34) holds. It follows from this inequality and from (4.1.32) that $C_{p, \ell}$ satisfies the bounds (1.2.8) (or (1.2.9)) for $m=0, k=0,1$. Iterating the reasonning, we conclude that $C_{p, \ell}$ is in $\widetilde{H \Psi}_{p}^{0, \nu}$ as wanted.

End of proof of Theorem 3.1.1. We consider the expression (4.1.24) of $G \circ \chi^{-1}$. The first term in the right hand side is given by (4.1.25). If $\sigma$ is large enough, the contributions $H_{\ell}$ with $\ell \geq P$ belong to $H \mathcal{F}_{\ell}^{1, \nu^{\prime}}\left(B_{\sigma}(R)\right)$ which is contained by the last remark following Definition 1.2.5 in $\mathcal{F}_{P}^{1, \nu}\left(B_{\sigma}(R)\right)$ if $\nu>\nu^{\prime}+\nu(\ell)-\nu(P)+\frac{1}{2}$. Since there are only finitely many $H_{\ell}$ 's, this gives a contribution of the form of $\tilde{G}_{P}$ given by (3.1.6) to $G \circ \chi^{-1}$. For $1 \leq \ell \leq P-1$, we have constructed $F_{\ell}$ in Lemma 4.1 .5 so that $\left\{F_{\ell}, G_{0}\right\}-H_{\ell}$ belongs to $H \mathcal{F}_{\ell, \mathrm{H}}^{1, \nu}\left(B_{\sigma}(R)\right)+\mathcal{G}_{\ell}^{1-\infty, \nu+\infty}\left(B_{\sigma}(R)\right)$, if $\nu$ is taken large enough relatively to $\nu^{\prime}$. These terms give a contribution to $G_{L}+G_{\mathrm{H}}$ defined by (3.1.5), (3.1.4). To prove (3.1.3), we write, since $\chi$ is canonical,

$$
\left\{\Theta_{s}^{1} \circ \chi, G\right\}=\left\{\Theta_{s}^{1}, G \circ \chi^{-1}\right\} \circ \chi
$$

and since the sum in the right hand side of (4.1.24) gives to $G \circ \chi^{-1}$ a contribution of the form $G_{L}+G_{\mathrm{H}}+\tilde{G}_{P}$, we are left with showing that, if

$$
S=\frac{(-1)^{P}}{(P-1) !} \int_{0}^{1}(1-t)^{P-1}\left(\left(\operatorname{ad}^{P} F\right) \cdot G\right)(\Phi(t, U)) d t,
$$

then

$$
\left\{\Theta_{s}^{1}, S\right\} \circ \chi=\left\{\Theta_{s}^{1}, \tilde{G}_{P}\right\} \circ \chi+O\left(\|u\|_{H^{s}}^{P+2}\right)
$$


for some (new) contribution $\tilde{G}_{P}$ of form (3.1.6). The term $S$ may be written as a sum of expressions

$$
\int_{0}^{1}(1-t)^{P-1} H_{p}(\Phi(t, U)) d t
$$

where $p \geq P$ and $H_{p}$ is an expression of form (4.1.26), so belongs to $H \mathcal{F}_{p}^{1, \nu^{\prime}}\left(B_{\sigma}(R)\right)$, i.e. (4.1.39) may be written from

$$
\begin{aligned}
& \operatorname{Re} \int_{0}^{1}(1-t)^{P-1} \int_{X}\left(A_{p}(\Phi(t, U)) \phi(t, U)\right) \phi(t, U) d \mu d t \\
& \operatorname{Re} \int_{0}^{1}(1-t)^{P-1} \int_{X}\left(A_{p}(\Phi(t, U)) \phi(t, U)\right) \overline{\phi(t, U)} d \mu d t
\end{aligned}
$$

with $A_{p}$ in $H \Psi_{p}^{1, \nu^{\prime}}$. The first component $\phi$ of $\Phi$ is given, according to (4.1.17), by

$$
\phi(t, U)=u+B_{1}(t, U) u+B_{2}(t, U) \bar{u}+R(t, U)
$$

where $\left(B_{j}(t, \cdot)\right)_{t \in[0,1]}$ are bounded families of elements of $\Psi_{1}^{0, \bar{\nu}}\left(B_{s}(R)\right)$ for $j=1,2$ and where $(R(t, \cdot))_{t \in[0,1]}$ is a bounded family of elements of $\mathcal{R}_{\gamma, 2}^{0}\left(B_{s}(R)\right)$ (for $s$ large enough). Plugging this expression inside (4.1.40), and using Corollary 4.1.3 and Lemma 1.2.6, we see that (4.1.40) may be written as a linear combination of elements of $\mathcal{F}_{P}^{1, \nu}\left(B_{s}(R)\right.$ ) (for some large enough $\nu$ and some large enough $s$ ) and of quantities of type

$$
\int_{0}^{1} \int_{X}(C(t, U) u) R(t, U) d \mu d t, \int_{0}^{1} \int_{X}(C(t, U) R(t, U)) R(t, U) d \mu d t
$$

with $(C(t, \cdot))_{t \in[0,1]}$ in $\Psi_{p}^{1, \nu}\left(B_{s}(R)\right),(R(t, \cdot))_{t \in[0,1]}$ in $\mathcal{R}_{\gamma, 2}^{0}\left(B_{s}(R)\right)$, as well as similar expressions with $u, R$ replaced by $\bar{u}, \bar{R}$. We just need to check that the Poisson bracket between $\Theta_{s}^{1}$ and (4.1.41) is $O\left(\|U\|_{H^{s}}^{P+2}\right)$ to get (4.1.38). By assumption, $\left\|D \Theta_{s}^{1}(U)\right\|_{\mathcal{L}\left(H^{s}, \mathbb{R}\right)}=O\left(\|U\|_{H^{s}}\right)$, so that it is enough to check that if $H(U)$ is any of the expressions (4.1.41), $\nabla H$ belongs to $H^{s}$, with $H^{s}$-norm $O\left(\|U\|_{H^{s}}^{P+1}\right)$. In other words, we have to study, for $K$ in $H^{-s}$,

$$
\begin{aligned}
& \int_{X}(C(t, U) K) R(t, U) d \mu, \int_{X}\left(\left(d_{U} C(t, U) \cdot K\right) u\right) R(t, U) d \mu \\
& \int_{X}(C(t, U) u)\left(d_{U} R(t, U) \cdot K\right) d \mu, \int_{X}(C(t, U) R(t, U))\left(d_{U} R(t, U) \cdot K\right) d \mu \\
& \int_{X}\left(\left(d_{U} C(t, U) \cdot K\right) R(t, U)\right) R(t, U) d \mu
\end{aligned}
$$

(and similar expressions involving transposes). By (4.1.1), $R(t, U)$ is in $H^{2 s-\gamma} \subset H^{s+1}$ (if $s \geq s_{0}$ large enough), by (1.2.19), $\left(d_{U} C(t, U) \cdot K\right) u \in H^{-\nu(p)-\nu} \subset H^{-s-1},\left(d_{U} C(t, U) \cdot K\right) R(t, U) \in H^{-s}$, by (4.1.3), $d_{U} R(t, U) \cdot K \in H^{-s+1}$. This shows that all the above integrals are meaningful and vanish at least at order $P+2$ at zero. This concludes the proof. 


\subsection{Diagonalization of the remainder term}

The goal of this subsection is to prove Theorem 3.1.2. Theorem 3.1.1 has reduced ourselves to the study of a new Hamiltonian $G^{1}=G_{L}+G_{\mathrm{H}}+\tilde{G}_{P}$, that may be written

$$
G^{1}=G_{L}(U)+\int_{X}\left(\left(\Lambda_{\mathbf{m}}+A(U)\right) u\right) \bar{u} d \mu+\frac{1}{2} \int_{X}\left(B_{P}(U) u\right) u d \mu+\frac{1}{2} \int_{X}\left(\overline{B_{P}(U)} \bar{u}\right) \bar{u} d \mu
$$

where $A(U)=\sum_{j=1}^{P} A_{j}(U)$. By the last remark following Definition 1.2.5, we may assume that $A$ is in $\Psi_{1}^{1, \nu}\left(B_{s}(R)\right)$ (increasing the value of $\nu$ given by Theorem 3.1.1). Moreover, $B_{P}$ is in $\Psi_{P}^{1, \nu}\left(B_{s}(R)\right)$, and we may assume $A(U)^{*}=A(U),{ }^{t} B_{P}(U)=B_{P}(U)$. We denote by $A_{\mathrm{H}}(U)=\sum_{p=1}^{P-1} A_{p}(U)$ the part of $A$ made of homogeneous terms. Consider the matrices of para-differential operators

$$
M(U)=\left[\begin{array}{cc}
B_{P}(U) & \Lambda_{\mathbf{m}}+{ }^{t} A(U) \\
\Lambda_{\mathbf{m}}+A(U) & \overline{B_{P}(U)}
\end{array}\right], M_{\mathrm{H}}(U)=\left[\begin{array}{cc}
0 & \Lambda_{\mathbf{m}}+{ }^{t} A_{\mathrm{H}}(U) \\
\Lambda_{\mathbf{m}}+A_{\mathrm{H}}(U) & 0
\end{array}\right]
$$

so that

$$
\left(G_{\mathrm{H}}+\tilde{G}_{P}\right)(U)=\frac{1}{2} \int_{X}(M(U) \cdot U) U d \mu, G_{\mathrm{H}}(U)=\frac{1}{2} \int_{X}\left(M_{\mathrm{H}}(U) \cdot U\right) U d \mu .
$$

Let us start with a diagonalization lemma.

Lemma 4.2.1 There are $s_{0}$ large enough, $\tilde{\nu} \geq \nu$ large enough, $R>0$ and for $s \geq s_{0}$, operators $Q$ in $\Psi_{P}^{0, \tilde{\nu}}\left(B_{s}(R)\right) \otimes \mathcal{M}_{2}(\mathbb{R}), C$ in $\Psi_{P}^{1, \tilde{\nu}}\left(B_{s}(R)\right)$, with $C$ self-adjoint and symmetric, such that, if we set

$$
\Sigma(U)=\left[\begin{array}{cc}
\Lambda_{\mathbf{m}}+A(U)+C(U) & 0 \\
0 & -\Lambda_{\mathbf{m}}-{ }^{t} A(U)-C(U)
\end{array}\right]
$$

one has the relations

$$
\begin{aligned}
& { }^{t}(I+Q(U))^{t} J(I+Q(U))-{ }^{t} J \in \Psi_{P}^{-1, \tilde{\nu}}\left(B_{s}(R)\right) \otimes \mathcal{M}_{2}(\mathbb{R}) \\
& (I+Q(U))\left({ }^{t} J M(U)\right) J^{t}(I+Q(U))^{t} J-\Sigma(U) \in \Psi_{P}^{0, \tilde{\nu}}\left(B_{s}(R)\right) \otimes \mathcal{M}_{2}(\mathbb{R}) .
\end{aligned}
$$

Proof: Define.

$$
A^{1}=\frac{1}{2}(A+\bar{A}), A^{2}=\frac{1}{2}(A-\bar{A}), B^{1}=\frac{1}{2}\left(B_{P}+\bar{B}_{P}\right), B^{2}=\frac{1}{2 i}\left(B_{P}-\bar{B}_{P}\right) .
$$

The assumptions on $A, B$ imply

$$
\left(A^{1}\right)^{*}={ }^{t} A^{1}=A^{1},\left(A^{2}\right)^{*}=A_{2}=-{ }^{t} A^{2},\left(B^{1}\right)^{*}=B^{1},\left(B^{2}\right)^{*}=B^{2}
$$

so that

$$
{ }^{t} J M(U)=\left[\begin{array}{cc}
\Lambda_{\mathbf{m}}+A^{1}+A^{2} & B^{1}-i B^{2} \\
-B^{1}-i B^{2} & -\Lambda_{\mathbf{m}}-A^{1}+A^{2}
\end{array}\right] .
$$


By (i) of Proposition 2.2.6, there are principal symbols $a^{1}, a^{2}$ in $S_{1}^{1, \nu}\left(B_{s}(R), T^{*} X\right), b^{1}, b^{2}$ in $S_{P}^{1, \nu}\left(B_{s}(R), T^{*} X\right)$ of $A^{1}, A^{2}, B^{1}, B^{2}$ respectively, modulo $\Psi_{1}^{0, \tilde{\nu}}\left(B_{s}(R)\right)$ and $\Psi_{P}^{0, \tilde{\nu}}\left(B_{s}(R)\right)$ for $\tilde{\nu} \geq$ $\nu+M_{0}(3 d+4)$. Since $A^{1}, A^{2}, B^{1}, B^{2}$ (resp. $\left.A^{1}, B^{1}, B^{2}\right)$ are self-adjoint (resp. symmetric) we may assume by (iv) of Proposition 2.2.6 that $a^{1}, a^{2}, b^{1}, b^{2}$ are real valued and that $a^{1}=\left(a^{1}\right)^{\vee}$, $b^{1}=\left(b^{1}\right)^{\vee}, b^{2}=\left(b^{2}\right)^{\vee}$ (increasing eventually $\tilde{\nu}$ ). We denote by $\lambda \in S_{0}^{1,0}\left(T^{*} M\right)$ the principal symbol of $\Lambda_{\mathbf{m}}$, given in local coordinates by $\left(\mathbf{m}^{2}+g_{x}(\xi, \xi)\right)^{1 / 2}$ where $g$ is the metric, and set

$$
b=b^{1}+i b^{2}, c=\frac{b}{\lambda+a^{1}+\sqrt{\left(\lambda+a^{1}\right)^{2}+|b|^{2}}} .
$$

If $R$ is small enough, the definition is meaningful since $a^{1}, b$ vanish at $U=0$, and we get that $c$ is in $S_{P}^{0, \nu}\left(B_{s}(R), T^{*} X\right)$. We define

$$
\mathrm{Id}+q=\left(1-|c|^{2}\right)^{-1 / 2}\left[\begin{array}{cc}
1 & \bar{c} \\
c & 1
\end{array}\right]
$$

By a direct computation using that $c^{\vee}=c$, we get

$$
{ }^{t}\left(\mathrm{Id}+q^{\vee}\right)^{t} J(\mathrm{Id}+q)={ }^{t} J
$$

and, if we define the matrix of symbols $\mathfrak{m}$ by

$$
{ }^{t} J \mathfrak{m}=\left[\begin{array}{cc}
\lambda+a^{1}+a^{2} & b^{1}-i b^{2} \\
-\left(b^{1}+i b^{2}\right) & -\lambda-a^{1}+a^{2}
\end{array}\right],
$$

we obtain

$$
(\mathrm{Id}+q)\left({ }^{t} J \mathfrak{m}\right) J^{t}\left(\operatorname{Id}+q^{\vee}\right)^{t} J=\left[\begin{array}{cc}
\sqrt{\left(\lambda+a^{1}\right)^{2}-|b|^{2}}+a^{2} & 0 \\
0 & -\sqrt{\left(\lambda+a^{1}\right)^{2}-|b|^{2}}+a^{2}
\end{array}\right] .
$$

Actually, the eigenvalues of $\left({ }^{t} J \mathfrak{m}\right)$ are $a^{2} \pm \sqrt{\left(\lambda+a^{1}\right)^{2}-|b|^{2}}$ and $\left[\begin{array}{c}1 \\ -c\end{array}\right]$ (resp. $\left[\begin{array}{c}-\bar{c} \\ 1\end{array}\right]$ ) is an eigenvector associated to the first (resp. second) eigenvalue. If we set

$$
(\mathrm{Id}+p)=\left(1-|c|^{2}\right)^{-1 / 2}\left[\begin{array}{cc}
1 & -\bar{c} \\
-c & 1
\end{array}\right]
$$

$(\mathrm{Id}+p)^{-1}\left({ }^{t} J \mathfrak{m}\right)(\mathrm{Id}+p)$ equals the right hand side of (4.2.9). One has just to define $(\mathrm{Id}+q)=$ $(\mathrm{Id}+p)^{-1}$ and to use (4.2.8) to get (4.2.9).

By (ii) of proposition 2.2.6, we may find operators $E, F$ in $\Psi_{P}^{0, \tilde{\nu}}\left(B_{s}(R)\right)$ whose principal symbols modulo $\Psi_{P}^{-1, \tilde{\nu}}\left(B_{s}(R)\right)$ are $\left(1-|c|^{2}\right)^{-1 / 2}$ and $\bar{c}\left(1-|c|^{2}\right)^{-1 / 2}$ respectively (taking again $\tilde{\nu}$ large enough). Since $c^{\vee}=c$, we may assume, using (iv) of Proposition 2.2.6, that $E={ }^{t} E, F={ }^{t} F$. We define $Q$ by

$$
I+Q(U)=\left[\begin{array}{ll}
E & F \\
\bar{F} & \bar{E}
\end{array}\right]
$$


Then (4.2.8) and the properties of symbolic calculus of (iii) of Proposition 2.2.6 imply that

$$
{ }^{t}(I+Q(U))^{t} J(I+Q(U))-{ }^{t} J \in \Psi_{P}^{-1, \tilde{\nu}}\left(B_{s}(R)\right) .
$$

Let $C$ be an element of $\Psi_{P}^{1, \tilde{\nu}}\left(B_{s}(R)\right)$, self-adjoint and symmetric, whose principal symbol modulo $\Psi_{P}^{0, \tilde{\nu}}\left(B_{s}(R)\right)$ is given by $\sqrt{\left(\lambda+a^{1}\right)^{2}-|b|^{2}}-\left(\lambda+a^{1}\right)$. Then (4.2.6), the definition (4.2.4) of $\Sigma$, the equality (4.2.9) and (iii) of proposition 2.2.6 imply that the second equality (4.2.5) holds (for $\tilde{\nu}$ large enough).

Before continuing the proof of Theorem 3.1.2, we write some properties that will be used repeatedly below. Consider $P$ an element of $\Psi_{p}^{m, \tilde{\nu}}\left(B_{s}(R)\right)$ for $m=0,1$. We apply (1.2.19) with $\sigma=s, \sigma^{\prime}=-s$ and the smoothness index denoted by $s$ in (1.2.19) taken to be $s$ or $s-1$. We get that if $H \in H^{-s}, d_{U} P(U) \cdot H$ belongs to $\mathcal{L}\left(H^{s}, H^{-s+1}\right)$ and to $\mathcal{L}\left(H^{s-1}, H^{-s}\right)$, if $s$ is taken large enough relatively to $\nu(p), \tilde{\nu}$. Moreover, the estimate

$$
\left\|d_{U} P(U) \cdot H\right\|_{\mathcal{L}\left(H^{s}, H^{-s+1}\right)}+\left\|d_{U} P(U) \cdot H\right\|_{\mathcal{L}\left(H^{s-1}, H^{-s}\right)} \leq C\|H\|_{H^{-s}}\|U\|_{H^{s}}^{p-1}
$$

holds. In the same way, again for $s$ large enough, if $H$ is in $H^{s-1}, d_{U} P(U) \cdot H$ is in $\mathcal{L}\left(H^{s}, H^{s-m}\right)$, with

$$
\left\|d_{U} P(U) \cdot H\right\|_{\mathcal{L}\left(H^{s}, H^{s-m}\right)} \leq C\|H\|_{H^{s-1}}\|U\|_{H^{s}}^{p-1} .
$$

If $P(U)=\tilde{P}(u, \ldots, u, \bar{u}, \ldots, \bar{u})$ with $\tilde{P}$ in $\widetilde{\mathcal{P}}_{p}^{m, \tilde{\nu}}$ for some $p \in \mathbb{N}^{*}, p \leq P-1$, estimates (1.2.7) show that (4.2.11), (4.2.12) hold. Actually, we shall also use similar estimates for the difference $\left(d_{U} P(U)-d_{U} P\left(U^{\prime}\right)\right) \cdot H$, replacing in the right hand side of (4.2.11), (4.2.12) $\|U\|_{H^{s}}^{p-1}$ by the quantity $\left(\|U\|_{H^{s}}+\left\|U^{\prime}\right\|_{H^{s}}\right)^{p-2}\left\|U-U^{\prime}\right\|_{H^{s}}$.

Lemma 4.2.2 There is $s_{0}>0$ and for any $s \geq s_{0}$, the map $U \rightarrow \psi(U)=(I+Q(U)) U$ defines a local diffeomorphism from a neighborhood of zero in $H^{s}$ to a neighborhood of zero in $H^{s}$, coming from a real diffeomorphism in real coordinates. If we define, for $V$ in a small enough neighborhood of zero in $H^{s}$,

$$
\widehat{\Sigma}(V)=\Sigma\left(\psi^{-1}(V)\right), \widehat{G}(V)=\frac{1}{2} \int_{X}(J \widehat{\Sigma}(V) V) V d \mu,
$$

then for any $U$ in a small enough neighborhood of zero in $H^{s}$

$$
\left\|d \psi(U) \cdot X_{\left[G_{\mathrm{H}}+\tilde{G}_{P}\right]}(U)-X_{\widehat{G}(\psi(U))}\right\|_{H^{s}}=O\left(\|U\|_{H^{s}}^{P+1}\right), U \rightarrow 0 .
$$

Proof: We shall call a "good term" any function $U \rightarrow S(U)$ defined on a neighborhood of zero in $H^{s}$, such that $\|S(U)\|_{H^{s}}=O\left(\|U\|_{H^{s}}^{P+1}\right), U \rightarrow 0$. It follows from (4.2.3) that $X_{\left[G_{\mathrm{H}}+\tilde{G}_{P}\right]}=$ $i^{t} J \nabla\left(G_{\mathrm{H}}+\tilde{G}_{P}\right)$ is given by

$$
\begin{aligned}
\int_{X} X_{\left[G_{\mathrm{H}}+\tilde{G}_{P}\right]}(U) \cdot H d \mu= & i d\left(G_{\mathrm{H}}+\tilde{G}_{P}\right)(U) \cdot(J H) \\
& =i \int_{X}\left({ }^{t} J M(U) \cdot U\right) H d \mu+\frac{i}{2} \int_{X}(d M(U) \cdot(J H) U) U d \mu
\end{aligned}
$$


for any $H$ in $C^{\infty}(X)$. We may write

$$
X_{\left[G_{\mathrm{H}}+\tilde{G}_{P}\right]}(U)=i^{t} J M(U) U+Z(U)
$$

where $Z(U)$ is defined by the equality

$$
\int_{X} Z(U) H d \mu=\frac{i}{2} \int_{X}(d M(U) \cdot(J H) U) U d \mu \text { for any } H .
$$

By (4.2.11), the right hand side is defined and continuous if $H$ is in $H^{-s}$, so that $Z$ is in $H^{s}$ and $\|Z(U)\|_{H^{s}}=O\left(\|U\|_{H^{s}}^{2}\right)$. By the inverse function theorem, $\psi$ is a local diffeomorphism at 0 , and the form (4.2.10) of $Q$ shows that $\psi$ sends a vector $\left[\frac{u}{u}\right]$ on a vector $\left[\frac{v}{v}\right]$ i.e. that it is induced by a local diffeomorphism in real coordinates. Compute

$$
\begin{array}{r}
d \psi(U) \cdot X_{\left[G_{\mathrm{H}}+\tilde{G}_{P}\right]}(U)=(I+Q(U)) i^{t} J M(U) U+(I+Q(U)) Z(U) \\
+\left(d Q(U) \cdot X_{\left[G_{\mathrm{H}}+\tilde{G}_{P}\right]}(U)\right) U .
\end{array}
$$

The fact that (4.2.16) belongs to $H^{s-1}$, and (4.2.12) with $m=0$, show that the last term in (4.2.18) is a good term. Since $Z(U)$ is in $H^{s}, Q(U) Z(U)$ is also a good term, so that

$$
d \psi(U) \cdot X_{\left[G_{\mathrm{H}}+\tilde{G}_{P}\right]}(U)=(I+Q(U)) i^{t} J M(U) U+Z(U)+S(U)
$$

where $S(U)$ is a good term. By the first equality (4.2.5), we may write

$$
U=J^{t}(I+Q(U))^{t} J\left(I+Q(U) U+S_{1}(U)\right.
$$

where $\left\|S_{1}(U)\right\|_{H^{s+1}} \leq C\|U\|_{H^{s}}^{P+1}$. Inserting this into the right hand side of (4.2.19) and using the second formula (4.2.5), we get

$$
d \psi(U) \cdot X_{\left[G_{\mathrm{H}}+\tilde{G}_{P}\right]}(U)=i \Sigma(U) \psi(U)+Z(U)+S(U)
$$

for a new good term $S(U)$. We define $\widehat{\Sigma}$ by (4.2.13). By (1.2.19), $d Q(U) \cdot H$ is in $\mathcal{L}\left(H^{s}, H^{s^{\prime}}\right)$ for any $s^{\prime}$ with $\left|s^{\prime}\right| \leq s$, any $H$ in $H^{s^{\prime}}$, if $s$ is large enough. Consequently, $d \psi(U)$ and $d \psi(U)^{-1}$ are bounded linear maps on $H^{s^{\prime}}$, for any $s^{\prime}$ with $\left|s^{\prime}\right| \leq s$, if $U$ stays in a small enough neighborhood of zero. Moreover, the operator norm of $d \psi(U)-\mathrm{Id}$ in these spaces is $O\left(\|U\|_{H^{s}}^{P}\right)$. A consequence of this and the definition of $\hat{\Sigma}$ is that $\widehat{\Sigma}(V)-\left[\begin{array}{cc}\Lambda_{\mathbf{m}} & 0 \\ 0 & -\Lambda_{\mathbf{m}}\end{array}\right]$ belongs to $\Psi_{1}^{1, \tilde{\nu}}\left(B_{s}(R)\right) \otimes \mathcal{M}_{2}(\mathbb{R})$ for $R>0$ small enough. Let us define $\widehat{M}(V)=J \widehat{\Sigma}(V)$. By (4.2.13), we may write

$$
X_{\widehat{G}}(V)=i \widehat{\Sigma}(V) V+\widehat{Z}(V)
$$

where $\widehat{Z}(V)$ is defined through the equality

$$
\int_{X} \widehat{Z}(V) \cdot H d \mu=\frac{i}{2} \int_{X}(d \widehat{M}(V) \cdot(J H) V) V d \mu \text { for any } H .
$$

To obtain (4.2.14), we must check that $d \psi(U) \cdot X_{\left[G_{\mathrm{H}}+\tilde{G}_{P}\right]}(U)-X_{\widehat{G}}(\psi(U))$ is a good term. By (4.2.20), (4.2.21), this difference is given by $Z(U)-\widehat{Z}(\psi(U))$, modulo a good term. To conclude 
the proof, we have to show, taking (4.2.17) and (4.2.22) into account, that, for any $H$ in $H^{-s}$, the modulus of

$$
\begin{aligned}
\int_{X}(Z(U)-\widehat{Z}(\psi(U))) H d \mu \\
\quad=\frac{i}{2} \int_{X}[((d M(U) \cdot(J H)) U) U-((d \widehat{M}(\psi(U))(J H)) \psi(U)) \psi(U)] d \mu
\end{aligned}
$$

is bounded from above by $C\|H\|_{H^{-s}}\|U\|_{H^{s}}^{P+1}$. By (4.2.11), $d \widehat{M}(\psi(U))(J H)$ is in $\mathcal{L}\left(H^{s}, H^{-s}\right)$ and $\|\psi(U)-U\|_{H^{s}}=O\left(\|U\|_{H^{s}}^{P}\right)$. Consequently, we are reduced to the study of

$$
\int_{X}(((d M(U)-d \widehat{M}(\psi(U))) \cdot(J H)) U) U d \mu .
$$

By definition of $\widehat{\Sigma}$ and the second relation (4.2.5)

$$
\widehat{M}(\psi(U))=J \Sigma(U)=J(I+Q(U))^{t} J M(U) J^{t}\left(I+Q(U)^{t} J+\mathfrak{R}(U)\right.
$$

with $\mathfrak{R}(U)$ in $\Psi_{P}^{0, \tilde{\nu}}\left(B_{s}(R)\right) \otimes \mathcal{M}_{2}(\mathbb{R})$. We decompose

$$
(d \widehat{M}(\psi(U))-d M(U)) \cdot(J H)=I+I I+I I I
$$

where

$$
\begin{gathered}
I=((d \widehat{M}(\psi(U))-d[\widehat{M}(\psi(U))]) \cdot(J H)=(d \widehat{M})(\psi(U))[\operatorname{Id}-d \psi(U)] \cdot J H \\
I I=J(d Q(U) \cdot(J H))^{t} J M(U) J^{t}(I+Q(U))^{t} J \\
\quad+J(I+Q(U))^{t} J M(U) J\left(d^{t} Q(U) \cdot(J H)\right)^{t} J+d \mathfrak{R}(U) \cdot J H \\
I I I=J(I+Q(U))^{t} J(d M(U) \cdot(J H)) J^{t}(I+Q(U))^{t} J-d M(U) \cdot(J H) .
\end{gathered}
$$

To finish to prove that $(4.2 .24)$ is $O\left(\|H\|_{H^{-s}}\|U\|_{H^{s}}^{P+1}\right)$, it remains to show that the $\mathcal{L}\left(H^{s}, H^{-s}\right)$ norm of I, II and III is $O\left(\|H\|_{H^{-s}}\|U\|_{H^{s}}^{P^{-1}}\right)$.

Since $\|d \psi(U)-\operatorname{Id}\|_{\mathcal{L}\left(H^{-s}, H^{-s}\right)}=O\left(\|U\|_{H^{s}}^{P}\right)$, the estimate of I follows from (4.2.11). The bound of II follows from (4.2.11) applied to $P=Q$, and from the fact that $d \mathfrak{R}(U) \cdot(J H)$ is in $\mathcal{L}\left(H^{s}, H^{-s}\right)$ with norm $O\left(\|H\|_{H^{-s}}\|U\|_{H^{s}}^{P-1}\right)$. Finally, the estimate of III follows again from (4.2.11), and from the fact that $\|Q(U)\|_{\mathcal{L}\left(H^{s^{\prime}}, H^{s^{\prime}}\right)}=O\left(\|U\|_{H^{s}}^{P}\right)$ for any $s^{\prime}$. This concludes the proof of the lemma.

End of the proof of Theorem 3.1.2: The left hand side of (3.1.7) is

$$
\left\{\Theta_{s}^{2} \circ \psi, G_{L}+G_{\mathrm{H}}+\tilde{G}_{P}\right\}=d \Theta_{s}^{2}(\psi(U)) \cdot d \psi(U) \cdot\left[X_{G_{L}}(U)+X_{\left[G_{\mathrm{H}}+\tilde{G}_{P}\right]}(U)\right] .
$$

The contribution of $G_{L}$ to the right hand side may be written

$$
\left\{\Theta_{s}^{2}, G_{L}\right\}(\psi(U))+d \Theta_{s}^{2}(\psi(U))\left[d \psi(U) X_{G_{L}}(U)-X_{G_{L}}(\psi(U))\right] .
$$

Since $d \Theta_{s}^{2} \in \mathcal{L}\left(H^{s}, \mathbb{R}\right)$, it is enough, to show that the last term is $O\left(\|U\|_{H^{s}}^{P+2}\right)$, to prove that

$$
d \psi(U) X_{G_{L}}(U)-X_{G_{L}}(\psi(U))=X_{G_{L}}(U)-X_{G_{L}}(\psi(U))+(d \psi(U)-\mathrm{Id}) \cdot X_{G_{L}}(U)
$$


is in $H^{s}$ and has $H^{s}$-norm $O\left(\|U\|_{H^{s}}^{P+1}\right)$. Since $G_{L}$ may be written $\sum_{p=1}^{P-1} G_{L, p}$, with $G_{L, p}$ in $\bigcap_{N} \mathcal{G}_{p}^{1-N, \nu+N}\left(B_{s}(R)\right) \subset \mathcal{G}_{p}^{0, \nu+1}\left(B_{s}(R)\right)$, we may write

$$
G_{L, p}=\operatorname{Re} \int_{X}\left(A_{L, p}(U) u\right) \bar{u} d \mu+\operatorname{Re} \int_{X}\left(B_{L, p} u\right) u d \mu
$$

with $A_{L, p}, B_{L, p}$ in $\mathcal{P}_{p}^{0, \nu+1}$. If we use (4.2.11), (4.2.12) applied to such operators (see the statement following these formulas), and the fact that $\|\psi(U)-U\|_{H^{s}}=O\left(\|U\|_{H^{s}}^{P+1}\right), \| d \psi(U)-$ $\operatorname{Id} \|_{\mathcal{L}\left(H^{ \pm s}, H^{ \pm s}\right)}=O\left(\|U\|_{H^{s}}^{P}\right)$, we get the wanted conclusion for (4.2.26).

Let us study the contribution of $G_{\mathrm{H}}+\tilde{G}_{P}$ to (4.2.25). We must prove that

$$
d \Theta_{s}^{2}(\psi(U)) \cdot d \psi(U) \cdot X_{\left[G_{\mathrm{H}}+\tilde{G}_{P}\right]}(U)-d \Theta_{s}^{2}(\psi(U)) \cdot X_{G_{\mathrm{H}}}(\psi(U))=O\left(\|U\|_{H^{s}}^{P+2}\right) .
$$

According to Lemma 4.2.2, and to the boundedness of $d \Theta_{s}^{2}$ (resp. $d \psi$ ) from $H^{s}$ to $\mathbb{R}$ (resp. to $\left.H^{s}\right)$, this will follow from the estimate

$$
d \Theta_{s}^{2}(\psi(U))\left[X_{\widehat{G}}(\psi(U))-X_{G_{\mathrm{H}}}(\psi(U))\right]=O\left(\|U\|_{H^{s}}^{P+2}\right), U \rightarrow 0 .
$$

Using expressions (4.2.3), (4.2.13) of $G_{\mathrm{H}}, \widehat{G}$, and setting $\Sigma_{\mathrm{H}}={ }^{t} J M_{\mathrm{H}}$ we write

$$
\widehat{G}(V)-G_{\mathrm{H}}(V)=\frac{1}{2} \int_{X}\left(J\left[\Sigma\left(\psi^{-1}(V)\right)-\Sigma_{\mathrm{H}}(V)\right] V\right) V d \mu
$$

and

$$
\begin{aligned}
& \Sigma\left(\psi^{-1}(V)\right)-\Sigma_{\mathrm{H}}(V) \\
& =\left[\begin{array}{cc}
A\left(\psi^{-1}(V)\right)-A_{\mathrm{H}}(V)+C\left(\psi^{-1}(V)\right) & 0 \\
0 & -\left({ }^{t} A\left(\psi^{-1}(V)\right)-{ }^{t} A_{\mathrm{H}}(V)+C\left(\psi^{-1}(V)\right)\right)
\end{array}\right]
\end{aligned}
$$

By definition of $A_{\mathrm{H}}$ (which is the sum of contributions to $A$ homogeneous of order up to $P-1$ ), $A-A_{\mathrm{H}}$ is in $\Psi_{P}^{1, \nu}\left(B_{s}(R)\right)$. Moreover, $C$ is in $\Psi_{P}^{1, \tilde{\nu}}\left(B_{s}(R)\right)$. Since we have seen that $\| d \psi^{-1}(V)-$ $\operatorname{Id} \|_{\mathcal{L}\left(H^{s^{\prime}}, H^{s^{\prime}}\right)}$ is $O\left(\|V\|_{H^{s}}^{P}\right)$ for any $s^{\prime}$ with $\left|s^{\prime}\right| \leq s$, we deduce that $A_{\mathrm{H}}(V)-A_{\mathrm{H}}\left(\psi^{-1}(V)\right)$ is in $\Psi_{P}^{1, \nu}\left(B_{s}(R)\right)$ and that $C\left(\psi^{-1}(V)\right)$ is in $\Psi_{P}^{1, \tilde{\nu}}\left(B_{s}(R)\right)$. Consequently, (4.2.29), (4.2.30) show that $\widehat{G}-G_{\mathrm{H}}$ belongs to $\mathcal{F}_{P, \mathrm{H}}^{1, \tilde{\nu}}\left(B_{s}(R)\right)$. The left hand side of (4.2.28) may be written $\left\{\Theta_{s}^{2}, \widehat{G}-G_{\mathrm{H}}\right\}(\psi(U))$. It follows from Lemma 3.2.6, and the assumption that $\Theta_{s}^{2}$ is a sum of elements of $H \mathcal{F}_{p, \mathrm{H}}^{2 s, \nu}\left(B_{s}(R)\right)+\mathcal{G}_{p}^{2 s-1, \nu}\left(B_{s}(R)\right)$ for some $\nu$ and $0 \leq p \leq P-1$, that this last term is $O\left(\|U\|_{H^{s}}^{P+2}\right)$ as wanted.

\subsection{Elimination of hyperbolic terms}

This section is devoted to the proof of Theorem 3.1.3. We prove first several helpful lemmas.

Remember that we denoted by $\left(\lambda_{n}^{2}\right)_{n \in \mathbb{N}^{*}}$ the eigenvalues of $-\Delta$ on $X=\mathbb{S}^{d}$ and that the mass parameter $\mathbf{m}$ has been chosen so that $(1.2 .27)$ holds. We set $\tilde{\Lambda}=\sqrt{-\Delta+\left(\frac{d-1}{2}\right)^{2}}$ so that $\tilde{\Lambda} \Pi_{n}=\left(n-1+\frac{d-1}{2}\right) \Pi_{n}$ for $n \in \mathbb{N}^{*}$. In particular, $t \rightarrow e^{i t \tilde{\Lambda}}$ is a $4 \pi$-periodic function. Moreover, $\Lambda_{\mathbf{m}}-\tilde{\Lambda}$ is a pseudo-differential operator of order -1 . 
Lemma 4.3.1 Let $\nu \in \mathbb{R}_{+}, p \in \mathbb{N}^{*}, \ell \in\{0, \ldots, p\}$ and $A^{\prime}$ be an element of the space $\widetilde{H \Psi}_{p}^{1, \nu}\left[M_{0}\right]$ introduced in Definition 1.2.2. Assume that $A^{\prime}$ satisfies the following condition (using the notations introduced before the statement of Theorem 1.2.8):

$$
\left(n_{0}, \ldots, n_{p+1}\right) \in Z^{\ell}(p) \Rightarrow \Pi_{n_{0}} A^{\prime}\left(\Pi_{n^{\prime}} \mathcal{U}\right) \Pi_{n_{p+1}}=0 .
$$

Denote $M_{1}=2 M_{0}(2 d+3), \nu_{1}=2 M_{1}$. There is a family $\left(C_{\alpha}(\mathcal{U})\right)_{\alpha \in \mathbb{Z}}$ of linear operators from $C^{\infty}(X)$ to $\mathcal{D}^{\prime}(X)$, such that, for any $t \in[0,4 \pi]$

$$
e^{-i t \tilde{\Lambda}} A^{\prime}(\mathcal{U}) e^{i t \tilde{\Lambda}}=\sum_{\alpha \in \mathbb{Z}} e^{i \alpha t / 2} C_{\alpha}(\mathcal{U})
$$

and, for any family of differential operators $P_{1}, \ldots, P_{k}$ of orders $d_{1}, \ldots, d_{k}$, and $N_{1}, N_{2}$ in $\mathbb{N}$, there is a constant $C>0$ such that, for any $\alpha \in \mathbb{Z}$, any $j, j^{\prime}$ in $\mathbb{N}$, any $n^{\prime}$ in $\left(\mathbb{N}^{*}\right)^{p}$, any $u_{1}, \ldots, u_{p}$ in $C^{\infty}(X)$

$$
\begin{aligned}
\left\|\Delta_{j} \operatorname{Ad}_{P_{1}} \cdots \operatorname{Ad}_{P_{k}} C_{\alpha}\left(\Pi_{n^{\prime}} \mathcal{U}\right) \Delta_{j^{\prime}}\right\|_{\mathcal{L}\left(L^{2}\right)} \leq \frac{C}{1+\alpha^{2}} 2^{-N_{1}\left|j-j^{\prime}\right|} 2^{j\left(1+\sum d_{\ell}-k\right)}\left|n^{\prime}\right|^{\nu(p)+\nu+\nu_{1}+M_{1} k} \\
\times\left(1+2^{-j}\left|n^{\prime}\right|\right)^{-N_{2}} \prod_{1}^{p}\left\|\Pi_{n_{\ell}} u_{\ell}\right\|_{L^{2}} .
\end{aligned}
$$

Moreover $C_{0}\left(\Pi_{n^{\prime}} \mathcal{U}\right) \equiv 0$ if $n^{\prime}$ is in $\underline{Z}^{\ell}(p) \subset\left(\mathbb{N}^{*}\right)^{p}$. Equation (4.3.3) implies in particular that $\sum_{\alpha} C_{\alpha}$ defines an element of $\widetilde{H \Psi}_{p}^{1, \nu+\nu_{1}}\left[M_{1}\right]$.

Proof: Using the $4 \pi$-time periodicity of the left hand side of (4.3.2), we write its Fourier series decomposition, with coefficients

$$
C_{\alpha}(\mathcal{U})=\frac{1}{4 \pi} \int_{0}^{4 \pi} e^{-i t \tilde{\Lambda}} A^{\prime}(\mathcal{U}) e^{i t \tilde{\Lambda}} e^{-i t \alpha / 2} d t
$$

Denote by $P_{k^{\prime}}(t)=e^{i t \tilde{\Lambda}} P_{k^{\prime}} e^{-i t \tilde{\Lambda}}, 1 \leq k^{\prime} \leq k$. By the Egorov theorem, this is a family of pseudodifferential operators of order $d_{k}$, with uniform estimates for $t \in[0,4 \pi]$. We may write, using two integrations by parts in $t$,

$$
\alpha^{2} \operatorname{Ad}_{P_{1}} \cdots \operatorname{Ad}_{P_{k}} C_{\alpha}(\mathcal{U})=\frac{1}{\pi} \int_{0}^{4 \pi} e^{-i t \tilde{\Lambda}} \operatorname{Ad}_{P_{1}(t)} \cdots \operatorname{Ad}_{P_{k}(t)} \operatorname{Ad}_{\tilde{\Lambda}}^{2} A^{\prime}(\mathcal{U}) e^{i t \tilde{\Lambda}} e^{-i t \alpha / 2} d t .
$$

By (iii) of Proposition 2.2.6, each commutator with $P_{k^{\prime}}(t)$ (resp. $\tilde{\Lambda}$ ) makes gain $d_{k^{\prime}}-1$ (resp. 0 ) units on the order of the operator, and makes lose $M_{1}=2 M_{0}(2 d+3)$ on the second index of this order. It follows that (4.3.3) holds, with the preceding value of $M_{1}$ and with $\nu_{1}=2 M_{1}$.

To get the statement concerning $C_{0}$, we compute

$$
\Pi_{n_{0}} C_{0}\left(\Pi_{n^{\prime}} \mathcal{U}\right) \Pi_{n_{p+1}}=\frac{1}{4 \pi} \int_{0}^{4 \pi} e^{-i t\left(n_{0}-n_{p+1}\right)} \Pi_{n_{0}} A^{\prime}\left(\Pi_{n^{\prime}} \mathcal{U}\right) \Pi_{n_{p+1}} d t .
$$

This quantity vanishes if $n_{0} \neq n_{p+1}$. If $n_{0}=n_{p+1}$, the condition $n^{\prime} \in \underline{Z}^{\ell}(p)$ is equivalent to $\left(n_{0}, n^{\prime}, n_{p+1}\right) \in Z^{\ell}(p)$, so that assumption (4.3.1) implies that the integrand vanishes. This concludes the proof. 
Lemma 4.3.2 Let $\mu \in \mathbb{R}, \nu \in \mathbb{R}_{+}, P \in \mathbb{N}, p \in \mathbb{N}, 1 \leq p \leq P-1, \ell \in\{0, \ldots, p\}, N \in \mathbb{N}$. There are positive numbers $\bar{\nu}(N), M_{0}(N)=M_{0}(4 d+6)^{N}$ such that the following holds:

(i) For any $A^{\prime}$ in $\widetilde{H \Psi}_{p}^{1, \nu}\left[M_{0}\right]$ satisfying (4.3.1), one may find operators $B$ (resp. S, resp. $R$ ) belonging to $\widetilde{H \Psi}_{p}^{1, \nu+\bar{\nu}(N)}\left[M_{0}(N)\right]$ (resp. $\widetilde{H \Psi}_{p}^{1-N, \nu+\bar{\nu}(N)}\left[M_{0}(N)\right], \widetilde{H \Psi}_{p}^{1, \nu+\bar{\nu}(N)}\left[M_{0}(N)\right]$ ), such that

$$
\begin{array}{r}
{\left[B(\mathcal{U}), \Lambda_{\mathbf{m}}\right]+\sum_{j=1}^{\ell} B\left(u_{1}, \ldots, \Lambda_{\mathbf{m}} u_{j}, \ldots, u_{p}\right)-\sum_{j=\ell+1}^{p} B\left(u_{1}, \ldots, \Lambda_{\mathbf{m}} u_{j}, \ldots, u_{p}\right)} \\
=A^{\prime}(\mathcal{U})+S(\mathcal{U})+\epsilon^{P} R(\mathcal{U}) .
\end{array}
$$

Moreover, $S$ satisfies (4.3.1).

(ii) Let $A^{\prime}$ be an element of $\widetilde{\mathcal{P}}_{p}^{\mu, \nu}$, satisfying (4.3.1). There is $B^{\prime}$ in $\widetilde{\mathcal{P}}_{p}^{\mu+L_{0}, \nu}$ such that

$$
\left[B(\mathcal{U}), \Lambda_{\mathbf{m}}\right]+\sum_{j=1}^{\ell} B\left(u_{1}, \ldots, \Lambda_{\mathbf{m}} u_{j}, \ldots, u_{p}\right)-\sum_{j=\ell+1}^{p} B\left(u_{1}, \ldots, \Lambda_{\mathbf{m}} u_{j}, \ldots, u_{p}\right)=A^{\prime}(\mathcal{U}) .
$$

The same conclusion holds for the equations

$\pm\left[\Lambda_{\mathbf{m}} B(\mathcal{U})+B(\mathcal{U}) \Lambda_{\mathbf{m}}\right]+\sum_{j=1}^{\ell} B\left(u_{1}, \ldots, \Lambda_{\mathbf{m}} u_{j}, \ldots, u_{p}\right)-\sum_{j=\ell+1}^{p} B\left(u_{1}, \ldots, \Lambda_{\mathbf{m}} u_{j}, \ldots, u_{p}\right)=A^{\prime}(\mathcal{U})$.

Proof: (i) Take $\theta \in C_{0}^{\infty}(\mathbb{R}), \theta \equiv 1$ close to zero, and define

$$
B_{1}\left(\Pi_{n^{\prime}} \mathcal{U}\right)=-i \int_{0}^{+\infty} e^{-i t \tilde{\Lambda}} A^{\prime}\left(\Pi_{n^{\prime}} \mathcal{U}\right) e^{i t \tilde{\Lambda}} e^{i t G_{\mathbf{m}}^{p, \ell}\left(n^{\prime}\right)} \theta(\epsilon t) d t
$$

where we denoted for short by $G_{\mathbf{m}}^{p, \ell}\left(n^{\prime}\right)$ the function $G_{\mathbf{m}}^{p, \ell}\left(\lambda_{n_{1}}, \ldots, \lambda_{n_{p}}\right)$ defined in (1.2.26). Using expansion (4.3.2), we write

$$
B_{1}\left(\Pi_{n^{\prime}} \mathcal{U}\right)=-i \sum_{\alpha \in \mathbb{Z}} a_{\alpha}\left(n^{\prime}, \epsilon\right) C_{\alpha}\left(\Pi_{n^{\prime}} \mathcal{U}\right)
$$

with

$$
a_{\alpha}\left(n^{\prime}, \epsilon\right)=\int_{0}^{+\infty} e^{i t\left(\frac{\alpha}{2}+G_{\mathbf{m}}^{p, \ell}\left(n^{\prime}\right)\right)} \theta(\epsilon t) d t .
$$

By (1.2.27), we know that $\left|\frac{\alpha}{2}+G_{\mathbf{m}}^{p, \ell}\left(n^{\prime}\right)\right| \geq c\left|n^{\prime}\right|^{-L_{0}}$ unless $\alpha=0$ and $n^{\prime} \in \underline{Z}^{\ell}(p)$. This last case is excluded since, by assumption and Lemma 4.3.1, $C_{0}\left(\Pi_{n^{\prime}} \mathcal{U}\right)=0$ when $n^{\prime} \in \underline{Z}^{\ell}(p)$. Consequently, an integration by parts shows that $\left|a_{\alpha}\left(n^{\prime}, \epsilon\right)\right| \leq C\left|n^{\prime}\right|^{L_{0}}$. Combining this with (4.3.3) and summing in $\alpha$, we see that $B_{1}$ satisfies the estimates of elements of $\widetilde{H \Psi}_{p}^{1, \nu+\nu_{1}+L_{0}}\left[M_{1}\right]$, where $M_{1}=2 M_{0}(2 d+3)$. Let us compute

$$
\begin{aligned}
-\left[\tilde{\Lambda}, B_{1}\left(\Pi_{n^{\prime}} \mathcal{U}\right)\right]+ & G_{\mathbf{m}}^{p, \ell}\left(n^{\prime}\right) B_{1}\left(\Pi_{n^{\prime}} \mathcal{U}\right) \\
=-\int_{0}^{+\infty} \frac{d}{d t}\left[e^{-i t \tilde{\Lambda}} A^{\prime}\left(\Pi_{n^{\prime}} \mathcal{U}\right) e^{i t \tilde{\Lambda}} e^{i t G_{\mathbf{m}}^{p, \ell}\left(n^{\prime}\right)}\right] \theta(\epsilon t) d t & =A^{\prime}\left(\Pi_{n^{\prime}} \mathcal{U}\right)+\epsilon^{P} R_{1}\left(\Pi_{n^{\prime}} \mathcal{U}\right)
\end{aligned}
$$


with

$$
R_{1}\left(\Pi_{n^{\prime}} \mathcal{U}\right)=\int_{0}^{+\infty} e^{-i t \tilde{\Lambda}} A^{\prime}\left(\Pi_{n^{\prime}} \mathcal{U}\right) e^{i t \tilde{\Lambda}} e^{i t G_{\mathbf{m}}^{p, \ell}\left(n^{\prime}\right)} \epsilon^{-P+1} \theta^{\prime}(\epsilon t) d t .
$$

Let $\theta_{1} \in C_{0}^{\infty}(] 0,+\infty[)$ such that $\theta_{1} \equiv \theta^{\prime}$ on $[0,+\infty[$. Using again decomposition (4.3.2), we write

$$
R_{1}\left(\Pi_{n^{\prime}} \mathcal{U}\right)=\sum_{\alpha \in \mathbb{Z}} C_{\alpha}\left(\Pi_{n^{\prime}} \mathcal{U}\right) \epsilon^{-P} \hat{\theta}_{1}\left(-\epsilon^{-1}\left(G_{\mathbf{m}}^{p, \ell}\left(n^{\prime}\right)+\frac{\alpha}{2}\right)\right) .
$$

By (1.2.27), $\epsilon^{-P}\left|\hat{\theta}_{1}\left(-\epsilon^{-1}\left(G_{\mathbf{m}}^{p, \ell}\left(n^{\prime}\right)+\frac{\alpha}{2}\right)\right)\right| \leq C\left|n^{\prime}\right|^{P L_{0}}$ so that (4.3.3) shows that $R_{1}^{\prime}$ belongs to the space $\widetilde{H \Psi}_{p}^{1, \nu+\nu_{1}+P L_{0}}\left[M_{1}\right]$. We deduce from (4.3.9) that

$$
\begin{aligned}
-\left[\Lambda_{\mathbf{m}}, B_{1}(\mathcal{U})\right]+\sum_{j=1}^{\ell} B_{1}\left(u_{1}, \ldots, \Lambda_{\mathbf{m}} u_{j}, \ldots, u_{p}\right)-\sum_{j=\ell+1}^{p+1} B_{1}\left(u_{1}, \ldots, \Lambda_{\mathbf{m}} u_{j}, \ldots, u_{p}\right) & \\
& =A^{\prime}(\mathcal{U})+\epsilon^{P} R_{1}(\mathcal{U})-\left[\Lambda_{\mathbf{m}}-\tilde{\Lambda}, B_{1}(\mathcal{U})\right] .
\end{aligned}
$$

Since $\Lambda_{\mathbf{m}}-\tilde{\Lambda}$ is of order $-1,\left[\Lambda_{\mathbf{m}}-\tilde{\Lambda}, B_{1}(\mathcal{U})\right]$ is in $\widetilde{H \Psi}_{p}^{0, \nu+\nu_{1}+L_{0}}\left[M_{1}\right]$, by Lemma 1.2.4, and satisfies condition (4.3.1), because $\Pi_{n}$ commutes to $\Lambda_{\mathbf{m}}-\tilde{\Lambda}$, and because $B_{1}$ satisfies (4.3.1), as follows from its expression (4.3.8) and the assumption on $A^{\prime}$. We see that we have solved an approximate version of (4.3.5), where $S$ is replaced by an operator $\left[\tilde{\Lambda}-\Lambda_{\mathbf{m}}, B_{1}(\mathcal{U})\right]$ of order 0 instead of $1-N$. Since condition (4.3.1) is satisfied by this operator, we may repeat the construction of $B_{1}$, defining successively $B_{2}, B_{3}, \ldots$ until $B=B_{1}+B_{2}+\cdots$ satisfies (4.3.5) with an error $S$ of the wanted order $1-N$. At each step, we have to increase the number of derivative losses on small frequencies $\nu$, and multiply the constant $M_{1}$ of estimate (4.3.3) by $2(2 d+3)$. We call $\bar{\nu}(N)$ the total loss at the end of the process, and get the wanted conclusion.

(ii) We compute (4.3.6) at $\Pi_{n^{\prime}} \mathcal{U}$, and compose at the left with $\Pi_{n_{p+1}}$ and at the right with $\Pi_{n_{0}}$. We get

$$
F_{\mathbf{m}}^{p, \ell}\left(\lambda_{n_{0}}, \ldots, \lambda_{n_{p+1}}\right) \Pi_{n_{p+1}} B\left(\Pi_{n^{\prime}} \mathcal{U}\right) \Pi_{n_{0}}=\Pi_{n_{p+1}} A^{\prime}\left(\Pi_{n^{\prime}} \mathcal{U}\right) \Pi_{n_{0}} .
$$

Since $A^{\prime}$ satisfies condition (4.3.1), $\left|F_{\mathbf{m}}^{p, \ell}\left(\lambda_{n_{0}}, \ldots, \lambda_{n_{p+1}}\right)\right|$ is bounded from below by the right hand side of the first estimate (1.2.27). Going back to the estimate (1.2.5) defining $\mathcal{P}_{p}^{\mu, \nu}$, we see that we may find a solution $B$ to (4.3.10) in $\mathcal{P}_{p}^{\mu+L_{0}, \nu}$. This concludes the proof since the same reasoning applies to (4.3.7).

Before proving Theorem 3.1.3, we recall some notations and results of [7]. Let $F=\sum_{p=1}^{P-1} F_{p}$, $H=\sum_{p=0}^{P-1} H_{p}$ be two functions defined on a neighborhood of zero in $H^{s}(X)$, which are sums of components homogeneous of order $p+2$. Assume also that $F, H$ have enough smoothness so that all Poisson brackets below are meaningful. We define a truncated Poisson bracket at order $P$ by

$$
\{F, H\}_{P}=\sum_{\substack{p+p^{\prime} \leq P-1 \\ p \geq 1, p^{\prime} \geq 0}}\left\{F_{p}, H_{p^{\prime}}\right\} .
$$


The iterates of $\{\cdot, \cdot\}_{P}$ are defined by

$$
\begin{aligned}
\operatorname{ad}_{P} F \cdot H & =\{F, H\}_{P} \\
\operatorname{ad}_{P}{ }^{j} F \cdot H & =\operatorname{ad}_{P} F \cdot\left(\operatorname{ad}_{P}{ }^{j-1} F \cdot H\right) .
\end{aligned}
$$

If $T$ is some indeterminate, we define

$$
\exp \left(\operatorname{Tad}_{P} F\right) \cdot H=\sum_{j=0}^{+\infty} \frac{T^{j}}{j !} \operatorname{ad}_{P}^{j} F \cdot H .
$$

Notice that the sum in the last series is finite, as all terms with $j \geq P$ vanish. Let $\Theta$ be another multi-linear expression in $U=(u, \bar{u})$. It is proved in Lemma 5.1.3 of [7] that

$$
\left\{\exp \left(\operatorname{Tad}_{P} F\right) \cdot \Theta, H\right\}_{P}=\exp \left(\operatorname{Tad}_{P} F\right) \cdot\left\{\Theta, \exp \left(-\operatorname{Tad}_{P} F\right) \cdot H\right\}_{P} .
$$

We define from $\Theta$ (resp. $H$ ) and from $F$ new functions, denoted formally by $\Theta \circ \chi_{F}^{P}$ (resp. $\left.H \circ\left(\chi_{F}^{P}\right)^{-1}\right)$, given by

$$
\begin{aligned}
\Theta \circ \chi_{F}^{P} & =\left(\exp \left(\operatorname{ad}_{P} F\right)\right) \cdot \Theta \\
H \circ\left(\chi_{F}^{P}\right)^{-1} & =\left(\exp \left(-\operatorname{ad}_{P} F\right)\right) \cdot H
\end{aligned}
$$

so that (4.3.14) may be written, taking $T=1$,

$$
\left\{\Theta \circ \chi_{F}^{P}, H\right\}_{P}=\left\{\Theta, H \circ\left(\chi_{F}^{P}\right)^{-1}\right\}_{P} \circ \chi_{F}^{P} .
$$

The main remaining steps in the proof of Theorem 3.1.3 will be to apply (4.3.16) with $\Theta$ replaced by $\Theta_{s}^{0}(u, \bar{u})=\int_{X}\left(\Lambda_{\mathbf{m}}^{2 s} u\right) \bar{u} d \mu, H$ replaced by the Hamiltonian $G_{L}+G_{\mathrm{H}}$ found in Theorem 3.1.2, and to construct a function $F$ so that $\left\{\Theta_{s}^{0},\left(G_{L}+G_{\mathrm{H}}\right) \circ\left(\chi_{F}^{P}\right)^{-1}\right\}_{P} \circ \chi_{F}^{P}=O\left(\epsilon^{P}\|u\|_{H^{s}}^{2}\right)$ (for $s$ large enough).

Let us fix some notations. We fix $P \in \mathbb{N}^{*}$ and $N$ large enough relatively to $P$. We shall need several constants indexed by $p=1, \ldots, P-1$, growing quickly enough. We take elements of $\left[1,+\infty\left[,\left(M_{i}(p)\right)_{1 \leq p \leq P-1},\left(\tilde{\nu}_{i}(p)\right)\right)_{1 \leq p \leq P-1}, i=1, \ldots, 4\right.$. We assume for $p, q \in\{1, \ldots, P-1\}$ such that $p+q \leq P-1$, and for some large $C_{0}$, depending on $P, N$,

$$
\begin{aligned}
& M_{1}(p) \geq C_{0} M_{2}(p) \geq C_{0}^{2} M_{3}(p) \geq C_{0}^{3} M_{4}(p) \geq C_{0}^{3} M_{0} \\
& M_{4}(p+q) \geq \max \left(M_{1}(p), M_{2}(q)\right) \\
& \min \left(\tilde{\nu}_{1}(p), \tilde{\nu}_{2}(q)\right)+C_{0}\left[M_{1}(p)+M_{1}(q)\right] \leq \tilde{\nu}_{4}(p+q) \\
& \tilde{\nu}_{j}(p) \geq \tilde{\nu}_{j+1}(p)+C_{0} M_{j+1}(p), j=1,2,3,
\end{aligned}
$$

where $M_{0}$ is the constant introduced in Definition 1.2.2 We assume moreover that, if $p \rightarrow \nu(p)$ is the function of the definition of classes $\widetilde{H \Psi}$, it satisfies, for $p, q$ in $\{1, \ldots, P-1\}$,

$$
\nu(p)+\nu(q)+\nu+\max \left(\tilde{\nu}_{1}(p), \tilde{\nu}_{2}(q)\right)+C_{0} \max \left(M_{1}(p), M_{2}(q)\right) \leq \tilde{\nu}_{4}(p+q) .
$$

Finally, we take two other functions $p \rightarrow N_{i}(p), i=1,2$, such that $N_{1}(1)+M_{0}=N_{2}(1)=N$ and that, for any $p, q \in\{1, \ldots, P-1\}$ with $p+q \leq P-1$,

$$
N_{2}(p+q) \leq N_{2}(q)-M_{0}-1, N_{2}(p+q) \leq N_{1}(p)-M_{0}-1, N_{1}(p) \leq N_{2}(p)-L_{0}
$$


(where $L_{0}$ is the exponent in (1.2.27)). If $N=N_{2}(1) \gg P$, we may find such $N_{i}(p)$ 's, which are moreover larger or equal to $P$ for $p=1, \ldots, P-1$. We may assume as well that $\tilde{\nu}_{4}(p) \geq N_{2}(p)$ for any $p=1, \ldots, P-1$, since it is always possible to increase the $\tilde{\nu}_{j}(p)$ in such a way they will continue to satisfy (4.3.17).

We use inclusions (3.2.12), (3.2.14) and (3.2.13) (with the constant $M_{0}$ of (3.2.10) replaced by $M_{1}(p)$ or $\left.M_{2}(q)\right)$ to get, taking into account assumptions (4.3.17), (4.3.18), (4.3.19), the inclusion

$$
\begin{aligned}
\left\{H_{p, \mathrm{H}}^{1, \nu+\tilde{\nu}_{1}(p)}\right. & \left(B_{s}(R)\right)\left[M_{1}(p)\right]+\mathcal{G}_{p}^{1-N_{1}(p), \nu+\tilde{\nu}_{1}(p)}\left(B_{s}(R)\right) \\
& \left.H_{q, \mathrm{H}}^{1, \nu+\tilde{\nu}_{2}(q)}\left(B_{s}(R)\right)\left[M_{2}(q)\right]+\mathcal{G}_{q}^{1-N_{2}(q), \nu+\tilde{\nu}_{2}(q)}\left(B_{s}(R)\right)\right\} \\
& \subset H^{1, \nu+\tilde{\nu}_{4}(p+q)}\left(B_{s}(R)\right)\left[M_{4}(p+q)\right]+\mathcal{G}_{p+q}^{1-N_{2}(p+q)-M_{0}, \nu+\tilde{\nu}_{4}(p+q)}\left(B_{s}(R)\right)
\end{aligned}
$$

for any $p>0, q>0$ such that $p, q, p+q$ are smaller than $P-1$. Let us prove:

Lemma 4.3.3 For any $p=1, \ldots, P-1$, there is an element

$$
F_{p} \in H \mathcal{F}_{p, \mathrm{H}}^{1, \nu+\tilde{\nu}_{1}(p)}\left(B_{s}(R)\right)\left[M_{1}(p)\right]+\mathcal{G}_{p}^{1-N_{1}(p), \nu+\tilde{\nu}_{1}(p)}\left(B_{s}(R)\right)
$$

satisfying

$$
\left\{F_{p}, G_{0}\right\} \in H \mathcal{F}_{p, \mathrm{H}}^{1, \nu+\tilde{\nu}_{2}(p)}\left(B_{s}(R)\right)\left[M_{2}(p)\right]+\mathcal{G}_{p}^{1-N_{2}(p), \nu+\tilde{\nu}_{2}(p)}\left(B_{s}(R)\right)
$$

such that, if $F=\sum_{p=1}^{P-1} F_{p}$

$$
\left\{\Theta_{s}^{0},\left(G_{L}+G_{\mathrm{H}}\right) \circ\left(\chi_{F}^{P}\right)^{-1}\right\}_{P}=\epsilon^{P}\left\{\Theta_{s}^{0}, R\right\},
$$

with $R$ in $\bigoplus_{p=1}^{P-1} H \mathcal{F}_{p, \mathrm{H}}^{1, \nu+\tilde{\nu}_{2}(p)}\left(B_{s}(R)\right)\left[M_{2}(p)\right]$.

Proof: By the definition (3.1.5), (3.1.4) of $G_{L}, G_{\mathrm{H}}$, we may write $G_{L}+G_{\mathrm{H}}=G_{0}+\sum_{p=1}^{P-1}\left(G_{L, p}+\right.$ $\left.G_{\mathrm{H}, p}\right)$ and $G_{L, p}+G_{\mathrm{H}, p} \in H \mathcal{F}_{p, \mathrm{H}}^{1, \nu}\left(B_{s}(R)\right)\left[M_{0}\right]+\bigcap_{N} \mathcal{G}_{p}^{1-N, \nu+N}\left(B_{s}(R)\right)$ for some $\nu$. In particular, $G_{L, p}+G_{\mathrm{H}, p}$ belongs to

$$
H \mathcal{F}_{p, \mathrm{H}}^{1, \nu+\tilde{\nu}_{4}(p)}\left(B_{s}(R)\right)\left[M_{4}(p)\right]+\mathcal{G}_{p}^{1-N_{2}(p), \nu+\tilde{\nu}_{4}(p)}\left(B_{s}(R)\right) .
$$

We compute $\left(G_{L}+G_{\mathrm{H}}\right) \circ\left(\chi_{F}^{P}\right)^{-1}$. By (4.3.15), (4.3.13), this may be written

$$
G_{0}-\sum_{p=1}^{P-1}\left\{F_{p}, G_{0}\right\}+\sum_{p=1}^{P-1} H_{p}
$$

where $H_{p}$ is a linear combination of quantities

$$
\left\{F_{p_{1}},\left\{F_{p_{2}}, \ldots,\left\{F_{p_{\gamma}}, G_{L, p_{\gamma+1}}+G_{\mathrm{H}, p_{\gamma+1}}\right\} \ldots\right\}\right\}
$$

where $p_{1}+\cdots+p_{\gamma+1}=p, p_{\beta} \geq 1$ if $1 \leq \beta \leq \gamma, p_{\gamma+1} \geq 0$ (with by convention the notation $\left.G_{L, 0}+G_{\mathrm{H}, 0}=G_{0}\right)$ and where $\gamma \geq 2$ if $p_{\gamma+1}=0$. Since $G_{L, p_{\gamma+1}}+G_{\mathrm{H}, p_{\gamma+1}}$ is in (4.3.24) with 
$p=p_{\gamma+1}$ and $F_{p_{\gamma}}$ satisfies (4.3.21) with $p=p_{\gamma}$, successive applications of inclusion (4.3.20) show that (4.3.25) belongs to (4.3.24) when $p_{\gamma+1}>0$. If $p_{\gamma+1}=0$, we use that $\gamma \geq 2$, so that we make use of (4.3.22) to reach the same conclusion. Consequently, $H_{p}$ belongs to (4.3.24), and the lemma will be proved if we construct $F_{p}$, satisfying (4.3.21) and (4.3.22) and such that

$$
\left\{\Theta_{s}^{0}, H_{p}-\left\{F_{p}, G_{0}\right\}\right\}=\epsilon^{P}\left\{\Theta_{s}^{0}, R_{p}\right\}
$$

with $R_{p}$ in $H \mathcal{F}_{p, \mathrm{H}}^{1, \nu+\tilde{\nu}_{2}(p)}\left(B_{s}(R)\right)\left[M_{2}(p)\right]$. We decompose $H_{p}=H_{p, \mathcal{F}}+H_{p, \mathcal{G}}$ according to $(4.3 .24)$ and write

$$
\begin{aligned}
H_{p, \mathcal{F}}(U) & =\operatorname{Re} \int_{X}\left(C_{p}(U) u\right) \bar{u} d \mu \\
H_{p, \mathcal{G}} & =\operatorname{Re} \int_{X}\left(E_{p, 0}(U) u\right) \bar{u} d \mu+\operatorname{Re} \int_{X}\left(E_{p, 1}(U) u\right) u d \mu
\end{aligned}
$$

with $C_{p}$ in $H \Psi_{p}^{1, \nu+\tilde{\nu}_{4}(p)}\left[M_{4}(p)\right], E_{p, 0}, E_{p, 1}$ in $\mathcal{P}_{p}^{1-N_{2}(p), \nu+\tilde{\nu}_{4}(p)} \subset \mathcal{P}_{p}^{1-N_{2}(p), \nu+\tilde{\nu}_{3}(p)}$. Coming back to the definition of these spaces, we decompose

$$
\begin{aligned}
C_{p}(U) & =\sum_{\ell=0}^{p} C_{p}^{\ell}(\underbrace{u, \ldots, u}_{\ell}, \bar{u}, \ldots, \bar{u}) \\
E_{p, i}(U) & =\sum_{\ell=0}^{p} E_{p, i}^{\ell}(\underbrace{u, \ldots, u}_{\ell}, \bar{u}, \ldots, \bar{u}), i=0,1
\end{aligned}
$$

with $C_{p}^{\ell}$ in $\widetilde{H \Psi}_{p}^{1, \nu+\tilde{\nu}_{4}(p)}\left[M_{4}(p)\right], E_{p, i}^{\ell}$ in $\widetilde{\mathcal{P}}_{p}^{1-N_{2}(p), \nu+\tilde{\nu}_{3}(p)}$. We may assume that, for a fixed large enough $C$,

$$
\Pi_{n_{0}} C_{p}^{\ell}\left(\Pi_{n^{\prime}} \mathcal{U}\right) \Pi_{n_{p+1}} \neq 0 \Rightarrow n_{0}, n_{p+1} \geq C\left|n^{\prime}\right|
$$

Actually, to ensure that condition, we may replace $C_{p}^{\ell}$ by

$$
\sum_{n^{\prime}} \sum_{j} \sum_{j^{\prime}} \mathbb{1}_{\left\{2^{j} \geq C\left|n^{\prime}\right|, 2^{j^{\prime}} \geq C\left|n^{\prime}\right|\right\}} \Delta_{j} C_{p}^{\ell}\left(\Pi_{n^{\prime}} \mathcal{U}\right) \Delta_{j^{\prime}}
$$

since, using formula (A.1) of the appendix, one checks that (4.3.30) is an element of the space $\widetilde{H \Psi}_{p}^{1, \nu+\tilde{\nu}_{4}(p)}\left[M_{4}(p)\right]$ satisfying (4.3.29). The difference between $C_{p}^{\ell}$ and (4.3.30) may be incorporated to $E_{p, 0}$ : taking into account that this difference belongs to

$$
\widetilde{H \Psi}_{p}^{1-N^{\prime}, \nu+\tilde{\nu}_{4}(p)+N^{\prime}}\left[M_{4}(p)\right] \subset \widetilde{\mathcal{P}}_{p}^{1-N^{\prime}, \nu+\tilde{\nu}_{4}(p)+N^{\prime}+2 M_{4}(p)}
$$

for any $N^{\prime}$, we take $N^{\prime}=N_{2}(p)$, and obtain that the perturbation of $E_{p, 0}$ will be in the class $\widetilde{\mathcal{P}}_{p}^{1-N_{2}(p), \nu+\tilde{\nu}_{3}(p)}$ (using the last inequality (4.3.17) with a large enough $C_{0}$ ).

Let us refine the decomposition of $C_{p}^{\ell}, E_{p, i}^{\ell}$. When $\ell \neq p / 2$, we set $E_{p, 0}^{\ell, \mathrm{Z}}=0$. When $\ell=p / 2$, we define

$$
E_{p, 0}^{\ell, \mathrm{Z}}=\sum_{\substack{\left\{n_{0}, \ldots, n_{\ell}\right\}=\\\left\{n_{\ell+1}, \ldots, n_{p+1}\right\}}} \Pi_{n_{0}} E_{p, 0}^{\ell}\left(\Pi_{n_{1}} u_{1}, \ldots, \Pi_{n_{p}} u_{p}\right) \Pi_{n_{p+1}} .
$$


In the same way, if $\ell+1 \neq p / 2$, we set $E_{p, 1}^{\ell, Z}=0$. If $\ell+1=p / 2$, we set

$$
E_{p, 1}^{\ell, \mathrm{Z}}=\sum_{\substack{\left\{n_{0}, \ldots, n_{\ell}, n_{p+1}\right\}=\\\left\{n_{\ell+1}, \ldots, n_{p}\right\}}} \Pi_{n_{0}} E_{p, 1}^{\ell}\left(\Pi_{n_{1}} u_{1}, \ldots, \Pi_{n_{p}} u_{p}\right) \Pi_{n_{p+1}} .
$$

Then $E_{p, i}^{\ell, \mathrm{Z}}$ are elements of $\widetilde{\mathcal{P}}_{p}^{1-N_{2}(p), \nu+\tilde{\nu}_{3}(p)}$. We define $E_{p, i}^{\ell, \mathrm{B}}=E_{p, i}^{\ell}-E_{p, i}^{\ell, \mathrm{Z}}$. In that way, $E_{p, 0}^{\ell, \mathrm{B}}$ satisfies (4.3.1) and $E_{p, 1}^{\ell, \mathrm{B}}$ satisfies

$$
\left\{n_{0}, \ldots, n_{\ell}, n_{p+1}\right\}=\left\{n_{\ell+1}, \ldots, n_{p}\right\} \Rightarrow \Pi_{n_{0}} E_{p, 1}^{\ell, \mathrm{B}}\left(\Pi_{n_{1}} u_{1}, \ldots, \Pi_{n_{p}} u_{p}\right) \Pi_{n_{p+1}}=0 .
$$

We perform a similar decomposition of $C_{p}^{\ell}=C_{p}^{\ell, \mathrm{Z}}+C_{p}^{\ell, \mathrm{B}}$ using formula (4.3.31). Let us show that $C_{p}^{\ell, \mathrm{Z}}$ belongs to $\widetilde{H \Psi}_{p}^{1, \nu+\tilde{\nu}_{3}(p)}\left[M_{3}(p)\right]$. Actually, because of condition (4.3.29), the property $\left\{n_{0}, \ldots, n_{\ell}\right\}=\left\{n_{\ell+1}, \ldots, n_{p+1}\right\}$ in the sum of type (4.3.31) defining $C_{p}^{\ell, Z}$ from $C_{p}^{\ell}$, is equivalent to $\left(n_{0}=n_{p+1}\right)$ and $\left\{n_{1}, \ldots, n_{\ell}\right\}=\left\{n_{\ell+1}, \ldots, n_{p}\right\}$. The definition of $C_{p}^{\ell, \mathrm{Z}}$ may be written equivalently as

$$
C_{p}^{\ell, \mathrm{Z}}\left(u_{1}, \ldots, u_{p}\right)=\sum_{\substack{\left\{n_{1}, \ldots, n_{\ell}\right\}=\\\left\{n_{\ell+1}, \ldots, n_{p}\right\}}} \frac{1}{4 \pi} \int_{0}^{4 \pi} e^{-i t \tilde{\Lambda}} C_{p}^{\ell}\left(\Pi_{n^{\prime}} \mathcal{U}\right) e^{i t \tilde{\Lambda}} d t
$$

We have seen in the proof of Lemma 4.3.1 that this time average belongs to $\widetilde{H \Psi}_{p}^{1, \nu+\tilde{\nu}_{3}(p)}\left[M_{3}(p)\right]$ (This follows from the fact that by (4.3.17), $M_{3}(p) \geq C_{0} M_{4}(p), \tilde{\nu}_{3}(p) \geq \tilde{\nu}_{4}(p)+C_{0} M_{4}(p)$, which are the requirement that the new constants $\left(\nu_{1}, M_{1}\right)$ in the right hand side of (4.3.3) have to satisfy). Set

$$
\begin{aligned}
C_{p}^{\mathrm{Z}}(U) & =\sum_{\ell=0}^{p} C_{p}^{\ell, \mathrm{Z}}(\underbrace{u, \ldots, u}_{\ell}, \bar{u}, \ldots, \bar{u}), E_{p, i}^{\mathrm{Z}}(U)=\sum_{\ell=0}^{p} E_{p, i}^{\ell, \mathrm{Z}}(\underbrace{u, \ldots, u}_{\ell}, \bar{u}, \ldots, \bar{u}) \\
C_{p}^{\mathrm{B}}(U) & =\left(C_{p}-C_{p}^{\mathrm{Z}}\right)(U), E_{p, i}^{\mathrm{B}}(U)=E_{p, i}(U)-E_{p, i}^{\mathrm{Z}}(U) \\
H_{p, \mathcal{F}}^{\mathrm{Z}}(U) & =\operatorname{Re} \int_{X}\left(C_{p}^{\mathrm{Z}}(U) u\right) \bar{u} d \mu, H_{p, \mathcal{G}}^{\mathrm{Z}}(U)=\operatorname{Re} \int_{X}\left(E_{p, 0}^{\mathrm{Z}}(U) u\right) \bar{u} d \mu+\operatorname{Re} \int_{X}\left(E_{p, 1}^{\mathrm{Z}}(U) u\right) u d \mu
\end{aligned}
$$

and define similarly $H_{p, \mathcal{F}}^{\mathrm{B}}(U), H_{p, \mathcal{G}}^{\mathrm{B}}(U)$. By construction and the definition of the Sobolev energy $\Theta_{s}^{0}(u, \bar{u})=\int_{X}\left(\Lambda_{\mathbf{m}}^{2 s} u\right) \bar{u} d \mu,\left\{\Theta_{s}^{0}, H_{p, \mathcal{F}}^{\mathrm{Z}}+H_{p, \mathcal{G}}^{\mathrm{Z}}\right\}=0$, so that equation (4.3.26) is equivalent to

$$
\left\{\Theta_{s}^{0}, H_{p, \mathcal{F}}^{\mathrm{B}}+H_{p, \mathcal{G}}^{\mathrm{B}}-\left\{F_{p}, G_{0}\right\}\right\}=\epsilon^{P}\left\{\Theta_{s}^{0}, R_{p}\right\} .
$$

Let us show that there is an element $R_{p}$ in $H \mathcal{F}_{p, \mathrm{H}}^{1, \nu+\tilde{\nu}_{2}(p)}\left(B_{s}(R)\right)\left[M_{2}(p)\right]$ and $F_{p}$ satisfying (4.3.21), (4.3.22) such that

$$
\left\{F_{p}, G_{0}\right\}=H_{p, \mathcal{F}}^{\mathrm{B}}+H_{p, \mathcal{G}}^{\mathrm{B}}-\epsilon^{P} R_{p} .
$$

We look for $F_{p}$ as

$$
F_{p}(U)=\operatorname{Re} \int_{X}\left(B_{p}(U) u\right) \bar{u} d \mu+\operatorname{Re} \int_{X}\left(D_{p, 0}(U) u\right) \bar{u} d \mu+\operatorname{Re} \int_{X}\left(D_{p, 1}(U) u\right) u d \mu
$$


where $B_{p}(U)=\sum_{\ell=0}^{p} B_{p}^{\ell}(u, \ldots, u, \bar{u}, \ldots, \bar{u}), D_{p, i}(U)=\sum_{\ell=0}^{p} D_{p, i}^{\ell}(u, \ldots, u, \bar{u}, \ldots, \bar{u})$, with $B_{p}^{\ell}$ in $\widetilde{H \Psi}_{p}^{1, \nu+\tilde{\nu}_{1}(p)}\left[M_{1}(p)\right], D_{p, i}^{\ell}$ in $\widetilde{\mathcal{P}}_{p}^{1-N_{1}(p), \nu+\tilde{\nu}_{1}(p)}$. Plugging (4.3.35) inside (4.3.34), we see that $B_{p}^{\ell}$ may be computed from $C_{p}^{\ell, \mathrm{B}}$ solving an equation of type (4.3.5). Applying (i) of Lemma 4.3.2, we obtain $B_{p}^{\ell}$ in $\widetilde{H \Psi}_{p}^{1, \nu+\tilde{\nu}_{1}(p)}\left[M_{1}(p)\right]$, using (4.3.17), and assuming that $C_{0}$ is larger than the loss $\bar{\nu}(N)$ of Lemma 4.3.2. We get the wanted property for $B_{p}^{\ell}$.

The remainder $S$ in the right hand side of (4.3.5) belongs to

$$
\widetilde{H \Psi}_{p}^{1-N, \nu+\tilde{\nu}_{3}(p)+\bar{\nu}(N)}\left[M_{3}(p)\right] \subset \widetilde{\mathcal{P}}_{p}^{1-N, \nu+\tilde{\nu}_{3}(p)+\bar{\nu}(N)+2 M_{3}(p)} .
$$

Since $N \geq N_{2}(p)$, we get an element of $\widetilde{\mathcal{P}}_{p}^{1-N_{2}(p), \nu+\tilde{\nu}_{2}(p)}$, if the constant $C_{0}$ in (4.3.17) has been taken large enough with respect to $N$. The contributions $E_{p, i}^{\ell, \mathrm{B}}$ belong to the same space. By (ii) of Lemma 4.3.2, we may construct $D_{p, 0}$ (resp. $\left.D_{p, 1}\right)$ in $\widetilde{\mathcal{P}}_{p}^{1-N_{2}(p)+L_{0}, \nu+\tilde{\nu}_{2}(p)}$ solving (4.3.6) (resp. (4.3.7)) when the right hand side $A^{\prime}$ is given by $E_{p, 0}^{\ell, \mathrm{B}}+S$ (resp $E_{p, 1}^{\ell, \mathrm{B}}$ ). If we assume $N_{1}(p) \leq N_{2}(p)-L_{0}$ as in (4.3.19), we get the wanted conclusion.

Finally, the term $\epsilon^{P} R$ in the right hand side of (4.3.5) belongs to $\epsilon^{P} \widetilde{H \Psi}_{p}^{1, \nu+\tilde{\nu}_{3}(p)+\bar{\nu}(N)}\left[M_{2}(p)\right] \subset$ $\epsilon^{P} \widetilde{H \Psi}_{p}^{1, \nu+\tilde{\nu}_{2}(p)}\left[M_{2}(p)\right]$. The contribution of this term to an integral of the form of the first one in (4.3.27) gives the last term in (4.3.34).

Proof of Theorem 3.1.3: We set $\Theta_{s}^{2}=\Theta_{s}^{0} \circ \chi_{F}^{P}$. This is linear combination of quantities

$$
\left\{F_{p_{1}},\left\{F_{p_{2}}, \ldots,\left\{F_{p_{\ell}}, \Theta_{s}^{0}\right\} \cdots\right\}\right\}, \ell \leq P-1,
$$

with $F_{p}$ satisfying $(4.3 .21), 1 \leq p \leq P-1$. Since we assume $N_{1}(p) \geq P$, we have

$$
F_{p} \in H \mathcal{F}_{p, \mathrm{H}}^{1, \nu}\left(B_{s}(R)\right)+\mathcal{G}_{p}^{1-P, \nu}\left(B_{s}(R)\right)
$$

for some large enough value of $\nu$. Moreover, $\Theta_{s}^{0}$ is in $H \mathcal{F}_{0, \mathrm{H}}^{2 s, 0}\left(B_{s}(R)\right)$. If $s \gg P$, it follows from inclusions (3.2.12), (3.2.13) and (3.2.14) that (4.3.36) is in $H \mathcal{F}_{p, \mathrm{H}}^{2 s, \nu}\left(B_{s}(R)\right)+\mathcal{G}_{p}^{2 s+\ell-P, \nu}\left(B_{s}(R)\right)$ for $p=p_{1}+\cdots+p_{\ell}$ and a new value of $\nu$, independent of $s$. Consequently $\Theta_{s}^{2}$ is in

$$
\bigoplus_{p=0}^{P-1}\left(H \mathcal{F}_{p, \mathrm{H}}^{2 s, \nu}\left(B_{s}(R)\right)+\mathcal{G}_{p}^{2 s-1, \nu}\left(B_{s}(R)\right)\right)
$$

for some $\nu$. $\mathrm{By}(4.3 .16)$

$$
\begin{aligned}
\left\{\Theta_{s}^{2}, G_{L}+G_{\mathrm{H}}\right\} & =\left\{\Theta_{s}^{2}, G_{L}+G_{\mathrm{H}}\right\}_{P}+\left(\left\{\Theta_{s}^{2}, G_{L}+G_{\mathrm{H}}\right\}-\left\{\Theta_{s}^{2}, G_{L}+G_{\mathrm{H}}\right\}_{P}\right) \\
& =\left\{\Theta_{s}^{0},\left(G_{L}+G_{\mathrm{H}}\right) \circ\left(\chi_{F}^{P}\right)^{-1}\right\}_{P} \circ \chi_{F}^{P}+\left(\left\{\Theta_{s}^{2}, G_{L}+G_{\mathrm{H}}\right\}-\left\{\Theta_{s}^{2}, G_{L}+G_{\mathrm{H}}\right\}_{P}\right) .
\end{aligned}
$$

The first term in the right hand side may be written according to (4.3.23) as $\epsilon^{P}\left\{\Theta_{s}^{0}, R\right\} \circ \chi_{F}^{P}$ with $R$ in $\bigoplus_{p=1}^{P-1} H \mathcal{F}_{p, \mathrm{H}}^{1, \nu+\tilde{\nu}_{2}(p)}\left(B_{s}(R)\right)$. Since $\Theta_{s}^{0}$ is in $H \mathcal{F}_{0, \mathrm{H}}^{2 s, 0}\left(B_{s}(R)\right)$, it follows from (3.2.12) that 
$\left\{\Theta_{s}^{0}, R\right\}$ is in $\bigoplus_{p=1}^{P-1} H \mathcal{F}_{p, \mathrm{H}}^{2 s, \nu}\left(B_{s}(R)\right)$ for some $\nu$ independent of $s$. Using (4.3.37) and again inclusions (3.2.12), (3.2.14), (3.2.13), we conclude that $\left\{\Theta_{s}^{0}, R\right\} \circ \chi_{F}^{P}$ belongs to $\bigoplus_{p=0}^{P-1}\left(H \mathcal{F}_{p, \mathrm{H}}^{2 s, \nu}\left(B_{s}(R)\right)+\right.$ $\left.\mathcal{G}_{p}^{2 s, \nu}\left(B_{s}(R)\right)\right)$. This implies that $\left\{\Theta_{s}^{0}, R\right\} \circ \chi_{F}^{P}(U)=O\left(\|U\|_{H^{s}}^{2}\right)$ if $s$ is large enough.

The last term in (4.3.39) may be written as a sum of expressions

$$
\left\{\theta_{p}, G_{L, q}+G_{\mathrm{H}, q}\right\}
$$

where $\theta_{p}$ is in $H \mathcal{F}_{p, \mathrm{H}}^{2 s, \nu}\left(B_{s}(R)\right)+\mathcal{G}_{p}^{2 s-1, \nu}\left(B_{s}(R)\right)$, and where $G_{L, q}+G_{\mathrm{H}, q}$ belongs to the space $H \mathcal{F}_{q, \mathrm{H}}^{1, \nu^{\prime}}\left(B_{s}(R)\right)+\mathcal{G}_{q}^{0, \nu^{\prime}}\left(B_{s}(R)\right)$ for some $\nu, \nu^{\prime}$ depending on $P$ but not on $s$, and with $p+q \geq P$. By (3.2.12), (3.2.13), (3.2.14), we obtain elements of $H \mathcal{F}_{p+q, \mathrm{H}}^{2 s, \nu^{\prime \prime}}\left(B_{s}(R)\right)+\mathcal{G}_{p+q}^{2 s, \nu^{\prime \prime}}\left(B_{s}(R)\right)$ for some new $\nu^{\prime \prime}$ independent of $s$. This implies that (4.3.40) is $O\left(\|U\|_{H^{s}}^{+2}\right)$, which is the wanted conclusion.

\section{A Appendix}

This appendix is devoted to an estimate that is used several times in the paper.

Proposition A.1 Let $P_{1}, \ldots, P_{k}$ be differential operators of order $d_{1}, \ldots, k_{k}$ on the manifold $X$. Let $Q, Q^{\prime}$ be two pseudo-differential operators of order $m, m^{\prime}$. For any $N$ in $\mathbb{N}$, there is $C>0$ and for any $j, j^{\prime}, j^{\prime \prime}$ in $\mathbb{N}$

$$
\left\|\Delta_{j^{\prime}}\left(\operatorname{Ad}_{P_{1}} \cdots \operatorname{Ad}_{P_{k}}\left(Q \Delta_{j} Q^{\prime}\right)\right) \Delta_{j^{\prime \prime}}\right\|_{\mathcal{L}\left(L^{2}\right)} \leq C 2^{-N\left[\left|j-j^{\prime}\right|+\left|j-j^{\prime \prime}\right|\right.} 2_{2^{j}}\left(m+m^{\prime}+\sum_{\ell=1}^{k} d_{\ell}-k\right) .
$$

If we prove

$$
\left\|\Delta_{j^{\prime}} \operatorname{Ad}_{P_{1}} \cdots \operatorname{Ad}_{P_{k}}\left(Q \Delta_{j} Q^{\prime}\right)\right\|_{\mathcal{L}\left(L^{2}\right)} \leq C 2^{-N\left|j-j^{\prime}\right|} 2^{j\left(m+m^{\prime}+\sum_{\ell=1}^{k} d_{\ell}-k\right)}
$$

we may deduce (A.1) from (A.2) by duality. To prove (A.2), we introduce, with the notations $(1.2 .2), \varphi_{1}(t)=\varphi(\sqrt{t}), \psi_{1}(t)=\psi(\sqrt{t})$, so that $\Delta_{j}=\varphi_{1}\left(-2^{-2 j} \Delta\right)$, for $j \geq 1, \Delta_{0}=\psi_{1}(-\Delta)$. We use the same letters $\varphi_{1}, \psi_{1}$ to denote almost holomorphic extensions of this functions to $\mathbb{C}$ (see Dimassi-Sjöstrand [11], Chapter 8). Then $\varphi_{1}, \psi_{1}$ are smooth functions on $\mathbb{C}, \varphi_{1}$ (resp. $\psi_{1}$ ) is supported in a domain $[a, b]+i[-c, c]$ with $0<a<b, c>0$ (resp. $a<0<b, c>0$ ), and $\bar{\partial} \varphi_{1}$, $\bar{\partial} \psi_{1}$ vanish at infinite order on $\mathbb{R}$. The Helffer-Sjöstrand formula [17], [11] Theorem 8.1 implies that

$$
\Delta_{j}=\frac{-1}{\pi} 2^{-2 j} \int_{\mathbb{C}}\left(\bar{\partial} \varphi_{1}\right)\left(2^{-2 j} \zeta\right)(\zeta+\Delta)^{-1} d \zeta d \bar{\zeta}
$$

when $j \geq 1$, and a similar expression for $\Delta_{0}$, with $\varphi_{1}$ replaced by $\psi_{1}$. We shall prove (A.2) when, more generally, $P_{1}, \ldots, P_{k}$ are pseudo-differential operators of order $d_{1}, \ldots, d_{k}$. Writing $\left[P_{j},(\zeta+\Delta)^{-1}\right]=-(\zeta+\Delta)^{-1}\left[P_{j}, \Delta\right](\zeta+\Delta)^{-1}$, we see that $\operatorname{Ad}_{P_{1}} \cdots \operatorname{Ad}_{P_{k}} \Delta_{j}$ may be written as a linear combination of expressions

$$
2^{-2 j} \int_{\mathbb{C}}\left(\bar{\partial} \varphi_{1}\right)\left(2^{-2 j} \zeta\right)\left[\prod_{\ell^{\prime}=1}^{k^{\prime}}(\zeta+\Delta)^{-1} Q_{\ell^{\prime}}\right](\zeta+\Delta)^{-1} d \zeta d \bar{\zeta}
$$


where $0 \leq k^{\prime} \leq k, Q_{\ell^{\prime}}, \ell^{\prime}=1, \ldots, k^{\prime}$ are pseudo-differential operators of order $D_{\ell^{\prime}}+2$ and $\sum_{1}^{k^{\prime}+1} D_{\ell^{\prime}}=\sum_{1}^{k} d_{\ell}-k$.

Lemma A.2 For any $s \in \mathbb{R}$

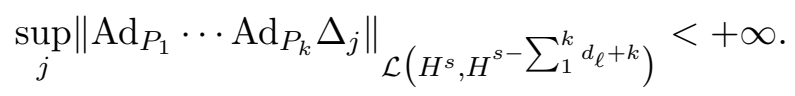

Proof: We note that for any $\sigma \in \mathbb{R}$

$$
\left\|(\zeta+\Delta)^{-1}\right\|_{\mathcal{L}\left(H^{\sigma}, H^{\sigma+2}\right)} \leq \frac{C(1+|\zeta|)}{|\operatorname{Im} \zeta|},\left\|(\zeta+\Delta)^{-1}\right\|_{\mathcal{L}\left(H^{\sigma}, H^{\sigma}\right)} \leq C|\operatorname{Im} \zeta|^{-1} .
$$

We deduce from (A.4), the above inequalities, the fact that on the support of $\varphi_{1}\left(2^{-2 j} \zeta\right),|\zeta| \leq$ $C 2^{2 j}$ and the vanishing of $\bar{\partial} \varphi_{1}$ at infinite order on $\mathbb{R}$, that (A.5) is bounded from above by the supremum in $j$ of quantities of the form

$$
C 2^{-2 j} \int_{\mathbb{C}}\left|\bar{\partial} \varphi_{1}\left(2^{-2 j} \zeta\right)\right| \frac{2^{2 j k^{\prime}}}{|\operatorname{Im} \zeta|^{k^{\prime}+1}} d \zeta d \bar{\zeta} \leq C 2^{2 j\left(k^{\prime}-1-N\right)} \int_{|\zeta| \leq C 2^{2 j}}|\operatorname{Im} \zeta|^{N-k^{\prime}-1} d \zeta d \bar{\zeta} \leq C
$$

for $0 \leq k^{\prime} \leq k$. This gives the lemma.

Proof of Proposition A.1: For $k \in \mathbb{N}, a \in \mathbb{R}$, denote by $\mathcal{E}_{k}(a)$ the family of operators which may be written as a finite sum of terms $Q_{0}\left(\operatorname{Ad}_{Q_{1}} \cdots \operatorname{Ad}_{Q_{k^{\prime}}} \Delta_{j}\right) Q_{k^{\prime}+1}$ where $k^{\prime} \leq k$ and $Q_{\ell}$, $\ell=0, \ldots, k^{\prime}+1$ are pseudo-differential operators such that $\sum_{\ell=0}^{k^{\prime}+1} \operatorname{deg} Q_{\ell}-k^{\prime}=a$. Let $Q, Q^{\prime}$ be a pseudo-differential operator of order $m, m^{\prime}$. Then

$$
\operatorname{Ad}_{P_{1}} \cdots \operatorname{Ad}_{P_{k}}\left[Q \Delta_{j} Q^{\prime}\right]=Q\left(\operatorname{Ad}_{P_{1}} \cdots \operatorname{Ad}_{P_{k}} \Delta_{j}\right) Q^{\prime} \text { modulo } \mathcal{E}_{k-1}\left(m+\sum_{1}^{k} d_{\ell}-k+m^{\prime}\right) .
$$

Actually, if (A.6) holds at order $k$, and if we consider another operator $P_{0}$ of order $d_{0}$

$$
\begin{aligned}
\operatorname{Ad}_{P_{0}} \operatorname{Ad}_{P_{1}} \cdots & \operatorname{Ad}_{P_{k}}\left[Q \Delta_{j} Q^{\prime}\right]=Q\left(\operatorname{Ad}_{P_{0}} \cdots \operatorname{Ad}_{P_{k}} \Delta_{j}\right) Q^{\prime}+\left[P_{0}, Q\right]\left(\operatorname{Ad}_{P_{1}} \cdots \operatorname{Ad}_{P_{k}} \Delta_{j}\right) Q^{\prime} \\
+ & Q\left(\operatorname{Ad}_{P_{1}} \cdots \operatorname{Ad}_{P_{k}} \Delta_{j}\right)\left[P_{0}, Q^{\prime}\right] \text { modulo }\left[P_{0}, \mathcal{E}_{k-1}\left(m+\sum_{1}^{k} d_{\ell}-k+m^{\prime}\right)\right] .
\end{aligned}
$$

We have just to notice that the second and third term in the right hand side are in $\mathcal{E}_{k}(m+$ $\left.\sum_{0}^{k} d_{\ell}-(k+1)+m^{\prime}\right)$, as well as the elements of $\left[P_{0}, \mathcal{E}_{k-1}\left(m+\sum_{1}^{k} d_{\ell}-k+m^{\prime}\right)\right]$.

Let us prove estimate (A.2). Assume first $j^{\prime}>j>0$ and write

$$
\Delta_{j^{\prime}} \operatorname{Ad}_{P_{1}} \cdots \operatorname{Ad}_{P_{k}}\left(Q \Delta_{j} Q^{\prime}\right)=2^{2 j N} \Delta_{j^{\prime}} \operatorname{Ad}_{P_{1}} \cdots \operatorname{Ad}_{P_{k}}\left(\tilde{Q} \tilde{\Delta}_{j} Q^{\prime}\right)
$$

where $\tilde{Q}=Q \Delta^{-N}$ and $\tilde{\Delta}_{j}=\tilde{\varphi}\left(-2^{-2 j} \Delta\right)$ for a new $\tilde{\varphi} \in C_{0}^{\infty}(] 0,+\infty[)$. By (A.6), we may write (A.7) as the product of $2^{2 j N}$ times operators of the form $\Delta_{j^{\prime}} Q_{0}\left(\operatorname{Ad}_{Q_{1}} \cdots \operatorname{Ad}_{Q_{k^{\prime}}} \tilde{\Delta}_{j}\right) Q_{k^{\prime}+1}$, where $k^{\prime} \leq k$ and $\sum_{0}^{k^{\prime}+1} \operatorname{deg} Q_{\ell}-k^{\prime}=-2 N+m+\sum_{1}^{k} d_{\ell}+m^{\prime}-k$. By Lemma A.2

$$
\begin{aligned}
\left\|\Delta_{j^{\prime}} Q_{0}\left(\operatorname{Ad}_{Q_{1}} \cdots \operatorname{Ad}_{Q_{k^{\prime}}} \tilde{\Delta}_{j}\right) Q_{k^{\prime}+1} u\right\|_{L^{2}} & \leq C 2^{-j^{\prime} \sigma}\left\|Q_{0}\left(\operatorname{Ad}_{Q_{1}} \cdots \operatorname{Ad}_{Q_{k^{\prime}}} \tilde{\Delta}_{j}\right) Q_{k^{\prime}+1} u\right\|_{H^{\sigma}} \\
& \leq C 2^{-j^{\prime} \sigma}\|u\|_{H^{\sigma-2 N+m+\sum_{1}} d_{d_{\ell}+m^{\prime}-k}} .
\end{aligned}
$$


We take $\sigma=-m-\sum_{1}^{k} d_{\ell}-m^{\prime}+2 N+k$ to conclude that

$$
\left\|\Delta_{j^{\prime}} \operatorname{Ad}_{P_{1}} \cdots \operatorname{Ad}_{P_{k}}\left(Q \Delta_{j} Q^{\prime}\right)\right\|_{\mathcal{L}\left(L^{2}\right)} \leq C_{N} 2^{2\left(j-j^{\prime}\right) N+j^{\prime}\left(m+\sum_{1}^{k} d_{\ell}+m^{\prime}-k\right)}
$$

for any $N$. When $j<j^{\prime}$, we perform the same computation taking $\tilde{Q}=Q \Delta^{N}$. This concludes the proof.

\section{References}

[1] D. Bambusi: Birkhoff normal form for some nonlinear PDEs, Comm. Math. Phys. 234 (2003), no. 2, 253-285.

[2] D. Bambusi, J.-M. Delort, B. Grébert and J. Szeftel: Almost global existence for Hamiltonian semi-linear Klein-Gordon equations with small Cauchy data on Zoll manifolds, Comm. Pure Appl. Math. 60 (2007), no. 11, 1665-1690.

[3] D. Bambusi and B. Grébert: Birkhoff normal form for partial differential equations with tame modulus, Duke Math. J. 135 (2006), no. 3, 507-567.

[4] R. Beals: Characterization of pseudodifferential operators and applications, Duke Math. J. 44 (1977), no. 1, 45-57.

[5] J. Bourgain: Construction of approximative and almost periodic solutions of perturbed linear Schrödinger and wave equations, Geom. Funct. Anal. 6 (1996), no. 2, 201-230.

[6] J.-M. Delort: On long time existence for small solutions of semi-linear Klein-Gordon equations on the torus, J. Anal. Math. 107 (2009), 161-194.

[7] J.-M. Delort: A quasi-linear Birkhoff normal forms method. Application to the quasi-linear Klein-Gordon equation on $\mathbb{S}^{1}$, to appear, Astérisque.

[8] J.-M. Delort and J. Szeftel: Long-time existence for small data nonlinear Klein-Gordon equations on tori and spheres, Internat. Math. Res. Notices (2004), no. 37, 1897-1966.

[9] J.-M. Delort and J. Szeftel: Long-time existence for semi-linear Klein-Gordon equations with small Cauchy data on Zoll manifolds, Amer. J. Math. 128 (2006), no. 5, 1187-1218.

[10] J.-M. Delort and J. Szeftel: Bounded almost global solutions for non Hamiltonian semilinear Klein-Gordon equations with radial data on compact revolution hypersurfaces, Ann. Inst. Fourier (Grenoble) 56 (2006), no. 5, 1419-1456.

[11] M. Dimassi and J. Sjöstrand: Spectral asymptotics in the semi-classical limit, London Mathematical Society Lecture Note Series, 268. Cambridge University Press, Cambridge, 1999. xii+227 pp.

[12] D. Fang and Q. Zhang: Long-time existence for semi-linear Klein-Gordon equations on tori, J. Differential Equations 249 (2010), no. 1, 151-179. 
[13] P. Germain: Global solutions for coupled Klein-Gordon equations with different speeds, to appear, Ann. Inst. Fourier (Grenoble).

[14] P. Germain, N. Masmoudi and J. Shatah: Global solutions for 3D quadratic Schrödinger equations, Int. Math. Res. Not. IMRN 2009, no. 3, 414-432.

[15] P. Germain, N. Masmoudi and J. Shatah: Global solutions for 2D quadratic Schrödinger equations, to appear, J. Math. Pures Appl.

[16] B. Grébert: Birkhoff normal form and Hamiltonian PDEs. Partial differential equations and applications, 1-46, Sémin. Congr., 15, Soc. Math. France, Paris, 2007.

[17] B. Helffer and J. Sjöstrand: Équation de Schrödinger avec champ magnétique et équation de Harper, Schrödinger operators (Sønderborg, 1988), 118-197, Lecture Notes in Phys., 345, Springer, Berlin, (1989).

[18] S. Klainerman: Global existence of small amplitude solutions to nonlinear Klein-Gordon equations in four space-time dimensions, Comm. Pure Appl. Math. 38, (1985) 631-641.

[19] J. Shatah: Normal forms and quadratic nonlinear Klein-Gordon equations. Comm. Pure Appl. Math. 38 (1985), no. 5, 685-696.

[20] Q. Zhang: Long-time existence for semi-linear Klein-Gordon equations with quadratic potential, Comm. Partial Differential Equations 35 (2010), no. 4, 630-668. 